\title{
A 2-CATEGORICAL EXTENSION OF ETINGOF-KAZHDAN QUANTISATION
}

\author{
ANDREA APPEL AND VALERIO TOLEDANO LAREDO
}

\begin{abstract}
Let $k$ be a field of characteristic zero. In [14], Etingof and Kazhdan construct a quantisation $U_{\hbar} \mathfrak{b}$ of any Lie bialgebra $\mathfrak{b}$ over $\mathrm{k}$, which depends on the choice of an associator $\Phi$. They prove moreover that this quantisation is functorial in $\mathfrak{b}$ [15]. Remarkably, the quantum group $U_{\hbar} \mathfrak{b}$ is endowed with a Tannakian equivalence $F_{\mathfrak{b}}$ from the braided tensor category of Drinfeld-Yetter modules over $\mathfrak{b}$, with deformed associativity constraints given by $\Phi$, to that of Drinfeld-Yetter modules over $U_{\hbar} \mathfrak{b}$ [16]. In this paper, we prove that the equivalence $F_{\mathfrak{b}}$ is functorial in $\mathfrak{b}$.
\end{abstract}

\section{Contents}

1. Introduction

2. Etingof-Kazhdan quantisation

3. Tensor structures on restriction functors 19

4. Quantisation of Verma modules 30

5. Quantisation of split pairs of Lie bialgebras 40

6. Universal constructions 46

7. Universal relative constructions $\quad 55$

8. Alternative constructions 63

Appendix A. Quantum double and Drinfeld-Yetter modules 66

$\begin{array}{ll}\text { References } & 70\end{array}$

The first author was supported in part through the NSF grant DMS-1255334, and the second through the NSF grant DMS-1505305. 


\section{INTRODUCTION}

1.1. Let $\mathrm{k}$ be a field of characteristic zero. In [14], Etingof and Kazhdan construct a quantisation $U_{\hbar} \mathfrak{b}$ of any Lie bialgebra $\mathfrak{b}$ over $k$. The quantisation depends on the choice of an associator $\Phi$, and has a number of remarkable properties: it is functorial in $\mathfrak{b}$ [15], compatible with taking doubles and duals [12] and, when $\mathfrak{b}$ is a symmetrisable Kac-Moody algebra with its standard bialgebra structure, coincides

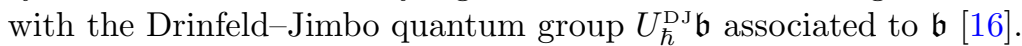

The quantum group $U_{\hbar} \mathfrak{b}$ is also compatible with another basic operation, namely taking the tensor category of Drinfeld-Yetter modules (see $\S 1.2-\S 1.3$ below for definitions). Specifically, it is endowed with a braided tensor equivalence

$$
\widetilde{F}_{\mathfrak{b}}: \mathrm{DY}_{\mathfrak{b}}^{\Phi} \longrightarrow \mathrm{DY}_{U_{\hbar} \mathfrak{b}}^{\mathrm{adm}}
$$

where $D Y_{\mathfrak{b}}^{\Phi}$ is the category of Drinfeld-Yetter modules over the Lie bialgebra $\mathfrak{b}$, with deformed associativity constraints given by $\Phi$, and $D Y_{U_{\hbar} \mathfrak{b}}^{\text {adm }}$ is the category of admissible Drinfeld-Yetter modules over $U_{\hbar} \mathfrak{b}$ [16]. If $\mathfrak{g}$ is a symmetrisable KacMoody algebra with negative Borel subalgebra $\mathfrak{b}$, this implies in particular the existence of an equivalence $E_{\mathfrak{g}}$ between category $\mathcal{O}$ representations of $\mathfrak{g}$ and those of the quantum group $U_{\hbar}^{\text {DJ } \mathfrak{g} \text {. }}$

Motivated by the theory of quasi-Coxeter algebras and categories [29, 2], we prove in this paper that the equivalence $\widetilde{F}_{\mathfrak{b}}$ is itself functorial with respect to $\mathfrak{b}$. This shows in particular that the Etingof-Kazhdan equivalence $E_{\mathfrak{g}}$ is compatible with respect to restriction to a standard Levi subalgebra.

1.2. A Drinfeld-Yetter module over a Lie bialgebra $\mathfrak{b}$ is a triple $\left(V, \pi, \pi^{*}\right)$ such that $\pi: \mathfrak{b} \otimes V \rightarrow V$ gives $V$ the structure of a left $\mathfrak{b}$-module, $\pi^{*}: V \rightarrow \mathfrak{b} \otimes V$ that of a right $\mathfrak{b}$-comodule, and $\pi, \pi^{*}$ satisfy a compatibility condition [15]. The latter is designed so as to give rise to a representation of the Drinfeld double $\mathfrak{g}_{\mathfrak{b}}=\mathfrak{b} \oplus \mathfrak{b}^{*}$ of $\mathfrak{b}$, with $\phi \in \mathfrak{b}^{*}$ acting on $V$ by $\phi \otimes \mathrm{id}_{V} \circ \pi^{*}$. If $\mathfrak{b}$ is finite-dimensional, the symmetric tensor category DY $\mathfrak{b}$ of such modules coincides with that of representations of $\mathfrak{g}_{\mathfrak{b}}$, with the coaction of $\mathfrak{b}$ on an object $V$ of the latter category given by $\pi^{*}(v)=\sum_{i} b_{i} \otimes b^{i} v$, where $\left\{b_{i}\right\},\left\{b^{i}\right\}$ are dual bases of $\mathfrak{b}$ and $\mathfrak{b}^{*}$. For an arbitrary $\mathfrak{b}$, DY $\mathfrak{b}$ coincides with the category of equicontinuous representations of $\mathfrak{g}_{\mathfrak{b}}$, which are roughly those carrying a locally finite action of $\mathfrak{b}^{*}[14]$.

1.3. A Drinfeld-Yetter module over a Hopf algebra $B$ is a triple $\left(V, \rho, \rho^{*}\right)$, where $\rho: B \otimes V \rightarrow V$ is a left $B$-module, $\rho^{*}: V \rightarrow B \otimes V$ a right $B$-comodule, and $\rho, \rho^{*}$ satisfy a suitable compatibility relation $[31,15]$. Such modules form a braided tensor category $\mathrm{DY}_{B}$, with commutativity constraints $\beta_{U, V}: U \otimes V \rightarrow V \otimes U$ given by

$$
\beta_{U, V}=(12) \circ\left(\rho_{U} \otimes \mathrm{id}_{V}\right) \circ(12) \circ\left(\mathrm{id}_{U} \otimes \rho_{V}^{*}\right) .
$$

If $B$ is finite-dimensional, the category $\mathrm{DY}_{B}$ coincides with that of representations of the quantum double of $B$ [7].

A similar statement holds if $B$ is a quantised universal enveloping algebra, that is a topological Hopf algebra over $\mathrm{k} \llbracket \hbar \rrbracket$ such that $B / \hbar B$ is a universal enveloping algebra $U \mathfrak{b}$. If $\mathfrak{b}$ is finite-dimensional, representations of the quantum double of $B$ coincide, as a braided tensor category, with the category DY ${ }_{B}^{\text {adm }}$ of admissible DrinfeldYetter modules over $B$, which are those for which the coaction $\rho^{*}: V \rightarrow B \otimes V$ factors through $B^{\prime} \otimes V$, where $B^{\prime} \subset B$ is the quantised formal group corresponding to $B$ defined in $[7,18]$. 
1.4. A crucial role in the quantisation of a Lie bialgebra $\mathfrak{b}$ is played by a deformation $D Y_{\mathfrak{b}}^{\Phi}$ of the braided tensor category $D Y_{\mathfrak{b}}$ over $\mathrm{K}=\mathrm{k} \llbracket \hbar \rrbracket$, where the commutativity and associativity constraints are respectively given by

$$
(12) \circ \exp (\hbar \Omega / 2) \quad \text { and } \Phi\left(\hbar \Omega_{12}, \hbar \Omega_{23}\right)
$$

with $\Omega \in \mathfrak{g}_{\mathfrak{b}} \widehat{\otimes} \mathfrak{g}_{\mathfrak{b}}$ the canonical element representing the inner product. Indeed, Etingof-Kazhdan construct a fiber functor $F_{\mathfrak{b}}: \mathrm{DY}_{\mathfrak{b}}^{\Phi} \rightarrow$ Vect $_{\mathfrak{k}}$, and obtain $U_{\hbar} \mathfrak{b}$ as a sub Hopf algebra of $\operatorname{End}\left(F_{\mathfrak{b}}\right)$ [14]. A remarkable feature of $U_{\hbar} \mathfrak{b}$ is that it is functorial with respect to $\mathfrak{b}[15]$, even though the intermediate steps in its construction, in particular taking the double of $\mathfrak{b}$ and considering the category $\mathrm{DY}_{\mathfrak{b}}$, are not.

The quantum group $U_{\hbar} \mathfrak{b}$ possesses another remarkable feature. Namely, in addition to acting on any $F_{\mathfrak{b}}(V), V \in \mathrm{DY}_{\mathfrak{b}}^{\Phi}$, it admits an admissible coaction on $F_{\mathfrak{b}}(V)$ which is compatible with its action. This gives rise to a Tannakian lift of $F_{\mathfrak{b}}$ as a braided tensor functor

$$
\widetilde{F}_{\mathfrak{b}}: \mathrm{DY}_{\mathfrak{b}}^{\Phi} \longrightarrow \mathrm{DY}_{U_{h} \mathfrak{b}}^{\text {adm }}
$$

where the right-hand side are the admissible Drinfeld-Yetter modules over $U_{\hbar} \mathfrak{b}$. Morever, $\widetilde{F}_{\mathfrak{b}}$ is an equivalence [16].

1.5. It is natural to ask whether the equivalence $\widetilde{F}_{\mathfrak{b}}$ is functorial with respect to $\mathfrak{b}$. The goal of this paper is to prove that this is indeed the case.

A proper formulation of this statement requires considering a different class of morphisms between Lie bialgebras, however, since taking Drinfeld-Yetter modules is not functorial in $\mathfrak{b}$. Let for this purpose $\operatorname{LBA}(k)$ be the (usual) category of Lie bialgebras over $k$, and $\operatorname{sLBA}(k)$ the category whose objects are Lie bialgebras, and morphisms are split embeddings $\mathfrak{a} \hookrightarrow \mathfrak{b}$ in $\operatorname{LBA}(k)$, that is

$$
\operatorname{Hom}_{\mathrm{sLBA}}(\mathfrak{a}, \mathfrak{b})=\left\{(i, p) \in \operatorname{Hom}_{\mathrm{LBA}}(\mathfrak{a}, \mathfrak{b}) \times \operatorname{Hom}_{\mathrm{LBA}}(\mathfrak{b}, \mathfrak{a}) \mid p \circ i=\operatorname{id}_{\mathfrak{a}}\right\} .
$$

A morphism in $\operatorname{sLBA}(k)$ gives rise to a restriction functor $\operatorname{Res}_{\mathfrak{a}, \mathfrak{b}}: \mathrm{DY}_{\mathfrak{b}} \longrightarrow \mathrm{DY}_{\mathfrak{a}}$ given by $\operatorname{Res}_{\mathfrak{a}, \mathfrak{b}}\left(V, \pi, \pi^{*}\right)=\left(V, \pi \circ(i \otimes \mathrm{id}),(p \otimes\right.$ id $\left.) \circ \pi^{*}\right) .{ }^{1}$ This functor admits a natural tensor structure

$$
J_{\mathfrak{a}, \mathfrak{b}}^{0}=\mathrm{id}: \operatorname{Res}_{\mathfrak{a}, \mathfrak{b}}\left(V_{1}\right) \otimes \operatorname{Res}_{\mathfrak{a}, \mathfrak{b}}\left(V_{2}\right) \longrightarrow \operatorname{Res}_{\mathfrak{a}, \mathfrak{b}}\left(V_{1} \otimes V_{2}\right)
$$

which clearly satisfies $\left(\operatorname{Res}_{\mathfrak{a}, \mathfrak{b}}, J_{\mathfrak{a}, \mathfrak{b}}^{0}\right) \circ\left(\operatorname{Res}_{\mathfrak{b}, \mathfrak{c}},, J_{\mathfrak{b}, \mathfrak{c}}^{0}\right)=\left(\operatorname{Res}_{\mathfrak{a}, \mathfrak{c}}, J_{\mathfrak{a}, \mathfrak{c}}^{0}\right)$ for any chain of split embeddings $\mathfrak{a} \hookrightarrow \mathfrak{b} \hookrightarrow \mathfrak{c}$. Thus, the assignment $\mathfrak{b} \rightarrow \mathrm{DY}_{\mathfrak{b}}$ extends to a contravariant functor from $\operatorname{sLBA}(\mathrm{k})$ to the $(1-)$ category $\mathrm{Cat}_{\mathrm{k}}^{\otimes}$ of $\mathrm{k}$-linear tensor categories.

1.6. In the presence of an associator $\Phi,\left(\operatorname{Res}_{\mathfrak{a}, \mathfrak{b}}, J_{\mathfrak{a}, \mathfrak{b}}^{0}\right)$ ceases to be a tensor functor, since the associativity constraints on $\mathrm{DY}_{\mathfrak{b}}^{\Phi}, \mathrm{DY}_{\mathfrak{a}}^{\Phi}$ are given by $\Phi_{\mathfrak{b}}=\Phi\left(\hbar \Omega_{12}^{\mathfrak{b}}, \hbar \Omega_{23}^{\mathfrak{b}}\right)$ and $\Phi_{\mathfrak{a}}=\Phi\left(\hbar \Omega_{12}^{\mathfrak{a}}, \hbar \Omega_{23}^{\mathfrak{a}}\right)$ respectively, and are therefore different. Our first main result asserts that $\operatorname{Res}_{\mathfrak{a}, \mathfrak{b}}$ can be endowed with a tensor structure $J_{\mathfrak{a}, \mathfrak{b}}$ compatible with those on $D Y_{\mathfrak{b}}^{\Phi}$ and $D Y_{\mathfrak{a}}^{\Phi}$. Moreover, with that tensor structure, the assignment $\mathfrak{b} \rightarrow \mathrm{DY}_{\mathfrak{b}}^{\Phi}$ extends to a 2-functor. The 2-functoriality accounts for the fact that, for a chain $\mathfrak{a} \hookrightarrow \mathfrak{b} \hookrightarrow \mathfrak{c}$, the composition $\left(\operatorname{Res}_{\mathfrak{a}, \mathfrak{b}}, J_{\mathfrak{a}, \mathfrak{b}}\right) \circ\left(\operatorname{Res}_{\mathfrak{b}, \mathfrak{c}}, J_{\mathfrak{b}, \mathfrak{c}}\right)$ is not equal to $\left(\operatorname{Res}_{\mathfrak{a}, \mathfrak{c}}, J_{\mathfrak{a}, \mathfrak{c}}\right)$, but only isomorphic to it via a coherent isomorphism.

\footnotetext{
${ }^{1}$ In terms of the Drinfeld doubles $\mathfrak{g}_{\mathfrak{a}}, \mathfrak{g}_{\mathfrak{b}}$ of $\mathfrak{a}, \mathfrak{b}$, a split embedding $\mathfrak{a} \hookrightarrow \mathfrak{b}$ corresponds to an isometric morphism of Lie algebras $j: \mathfrak{g}_{\mathfrak{a}} \rightarrow \mathfrak{g}_{\mathfrak{b}}$ such that $j(\mathfrak{a}) \subset \mathfrak{b}, j\left(\mathfrak{a}^{*}\right) \subset \mathfrak{b}^{*}$, and the transpose $j^{t}: \mathfrak{g}_{\mathfrak{b}} \rightarrow \mathfrak{g}_{\mathfrak{a}}$ restricts to morphisms of Lie algebras $\mathfrak{b} \rightarrow \mathfrak{a}$ and $\mathfrak{b}^{*} \rightarrow \mathfrak{a}^{*}$. Moreover, under the identification of the categories $\mathrm{DY}_{\mathfrak{a}}, \mathrm{DY}_{\mathfrak{b}}$ with those of equicontinuous modules over $\mathfrak{g}_{\mathfrak{a}}$ and $\mathfrak{g}_{\mathfrak{b}}$ respectively, the functor $\operatorname{Res}_{\mathfrak{a}, \mathfrak{b}}$ coincides with the restriction functor $j^{*}$.
} 
Specifically, consider sLBA $(k)$ as a 2-category with 2-morphisms given by equalities, and $\mathrm{Cat}_{\mathrm{K}}^{\otimes}$ as a 2-category in the usual way (1-morphisms are tensor functors, and 2-morphisms natural transformations). Then, the following holds for any associator $\Phi$

Theorem. There is a 2-functor ${ }^{2}$

$$
\mathrm{DY}^{\Phi}: \mathrm{sLBA}(\mathrm{k}) \longrightarrow \mathrm{Cat}_{\mathrm{K}}^{\otimes}
$$

which assigns

- to any Lie bialgebra $\mathfrak{b}$, the tensor category $\mathrm{DY}_{\mathfrak{b}}^{\Phi}$,

- to any split embedding $\mathfrak{a} \hookrightarrow \mathfrak{b}$, a tensor structure $J_{\mathfrak{a}, \mathfrak{b}}$ on the restriction functor $\operatorname{Res}_{\mathfrak{a}, \mathfrak{b}}: \mathrm{DY}_{\mathfrak{b}}^{\Phi} \rightarrow \mathrm{DY}_{\mathfrak{a}}^{\Phi}$,

- to any chain $\mathfrak{a} \hookrightarrow \mathfrak{b} \hookrightarrow \mathfrak{c}$, an isomorphism of tensor functors

$$
u_{\mathfrak{a}, \mathfrak{b}, \mathfrak{c}}:\left(\operatorname{Res}_{\mathfrak{a}, \mathfrak{b}}, J_{\mathfrak{a}, \mathfrak{b}}\right) \circ\left(\operatorname{Res}_{\mathfrak{b}, \mathfrak{c}}, J_{\mathfrak{b}, \mathfrak{c}}\right) \longrightarrow\left(\operatorname{Res}_{\mathfrak{a}, \mathfrak{c}}, J_{\mathfrak{a}, \mathfrak{c}}\right)
$$

in such a way that, for any chain $\mathfrak{a} \hookrightarrow \mathfrak{b} \hookrightarrow \mathfrak{c} \hookrightarrow \mathfrak{d}$, one has

$$
u_{\mathfrak{a}, \mathfrak{b}, \mathfrak{d}} \circ u_{\mathfrak{b}, \mathfrak{c}, \mathfrak{d}}=u_{\mathfrak{a}, \mathfrak{c}, \mathfrak{d}} \circ u_{\mathfrak{a}, \mathfrak{b}, \mathfrak{c}}
$$

as isomorphisms

$$
\left(\operatorname{Res}_{\mathfrak{a}, \mathfrak{b}}, J_{\mathfrak{a}, \mathfrak{b}}\right) \circ\left(\operatorname{Res}_{\mathfrak{b}, \mathfrak{c}}, J_{\mathfrak{b}, \mathfrak{c}}\right) \circ\left(\operatorname{Res}_{\mathfrak{c}, \mathfrak{d}}, J_{\mathfrak{c}, \mathfrak{d}}\right) \longrightarrow\left(\operatorname{Res}_{\mathfrak{a}, \mathfrak{d}}, J_{\mathfrak{a}, \mathfrak{d}}\right)
$$

1.7. Having established the correct functoriality of the assignment $\mathfrak{b} \rightarrow \mathrm{DY}_{\mathfrak{b}}^{\Phi}$, we turn now to the Etingof-Kazhdan equivalence $\widetilde{F}_{\mathfrak{b}}:$ DY $\mathfrak{b}_{\mathfrak{b}}^{\Phi} \rightarrow \mathrm{DY}_{U_{\hbar} \mathfrak{b}}^{\text {adm }}$. Our second main result is that $\widetilde{F}_{\mathfrak{b}}$ is functorial with respect to split embeddings and that, moreover, it fits within an isomorphism of 2 -functors sLBA(k) $\rightarrow \mathrm{Cat}_{\mathrm{K}}^{\otimes}$.

Specifically, let $\operatorname{sQUE}(k)$ be the category of quantised universal enveloping algebras over $\mathrm{k} \llbracket \hbar \rrbracket$, with morphisms given by split embeddings. Taking admissible Drinfeld-Yetter modules yields a functor $\mathrm{DY}^{\mathrm{adm}}: \mathrm{sQUE}(\mathrm{k}) \longrightarrow \mathrm{Cat}_{\mathrm{K}}^{\otimes}$, which assigns to a split embedding $A \hookrightarrow B$ the restriction functor $\operatorname{Res}_{A, B}: \mathrm{DY}_{B}^{\text {adm }} \rightarrow \mathrm{DY}_{A}^{\text {adm }}$ given by

$$
\operatorname{Res}_{A, B}\left(V, \rho, \rho^{*}\right)=\left(V, \rho \circ(i \otimes \mathrm{id}),(p \otimes \mathrm{id}) \circ \rho^{*}\right)
$$

and endowed with the trivial tensor structure. On the other hand, Etingof-Kazhdan quantisation gives rise to a functor $Q^{\Phi}: \operatorname{sLBA}(\mathrm{k}) \rightarrow \mathrm{sQUE}(\mathrm{k})$.

Theorem. There is an isomorphism of 2-functors

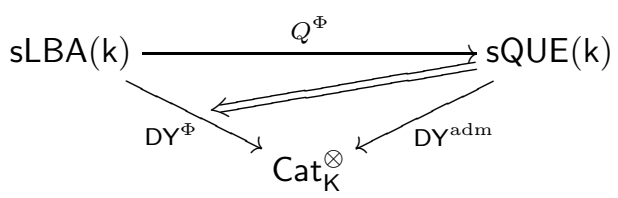

which assigns to a Lie bialgebra $\mathfrak{b} \in \operatorname{sLBA}(\mathrm{k})$ the tensor equivalence $\widetilde{F}_{\mathfrak{b}}: \mathrm{DY}_{\mathfrak{b}}^{\Phi} \rightarrow$ $\mathrm{DY}_{U_{\hbar} \mathfrak{b}}^{\mathrm{adm}}$. In particular,

\footnotetext{
${ }^{2}$ Strictly speaking $\mathrm{DY}^{\Phi}$ is a pseudo 2-functor in the terminology of [20] since it preserves the composition of 1-morphisms in $\operatorname{sLBA}(\mathrm{k})$ only up to the coherent isomorphisms $u_{\mathfrak{a}, \mathfrak{b}, \mathfrak{c}}$.
} 
- For any split embedding $\mathfrak{a} \hookrightarrow \mathfrak{b}$, there is a natural isomorphism $v_{\mathfrak{a}, \mathfrak{b}}$ making the following diagram commute

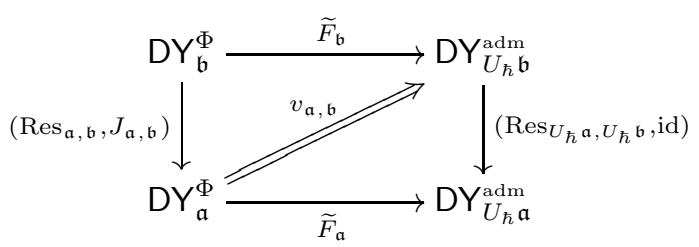

where $\left(\operatorname{Res}_{\mathfrak{a}, \mathfrak{b}}, J_{\mathfrak{a}, \mathfrak{b}}\right)$ is the tensor functor given by Theorem 1.6, and the functor $\operatorname{Res}_{U_{\hbar} \mathfrak{a}, U_{\hbar} \mathfrak{b}}$ is induced by the split embedding $U_{\hbar} \mathfrak{a} \hookrightarrow U_{\hbar} \mathfrak{b}$.

- For any chain of split embeddings $\mathfrak{a} \hookrightarrow \mathfrak{b} \hookrightarrow \mathfrak{c}$, the following diagram is commutative

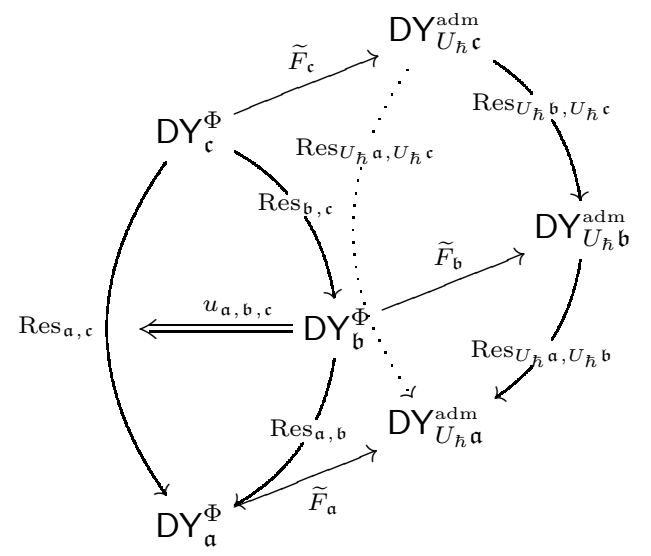

where $u_{\mathfrak{a}, \mathfrak{b}, \mathfrak{c}}$ is the isomorphism given by Theorem 1.6, the back 2-face is the identity, and the lateral 2-faces are the isomorphisms $v_{\mathfrak{a}, \mathfrak{c}}, v_{\mathfrak{b}, \mathfrak{c}}, v_{\mathfrak{a}, \mathfrak{b}} \cdot{ }^{3}$

1.8. We now outline the proofs of Theorems 1.6 and 1.7.

The construction of the tensor structure $J_{\mathfrak{a}, \mathfrak{b}}$ on the functor $\operatorname{Res}_{\mathfrak{a}, \mathfrak{b}}$ given by Theorem 1.6 is very much inspired by that of the Etingof-Kazhdan fiber functor $F_{\mathfrak{b}}$ [14], and reproduces the latter if $\mathfrak{a}=0$. The principle adopted in [14] is the following. In a k-linear monoidal category $\mathcal{C}$, a coalgebra structure on an object $C \in \operatorname{Obj}(\mathcal{C})$ induces a tensor structure on the Yoneda functor ${ }^{4}$

$$
h_{C}=\operatorname{Hom}_{\mathcal{C}}(C,-): \mathcal{C} \rightarrow \text { Vect }_{\mathrm{k}} .
$$

If, moreover, $\mathcal{C}$ is braided and $C_{1}, C_{2}$ are coalgebra objects in $\mathcal{C}$, then so is $C_{1} \otimes C_{2}$, and there is therefore a canonical tensor structure on $h_{C_{1} \otimes C_{2}}$.

If $\mathfrak{b}$ is a finite-dimensional Lie bialgebra, the forgetful functor DY $\mathfrak{b}_{\mathfrak{b}}=\operatorname{Rep}\left(\mathfrak{g}_{\mathfrak{b}}\right) \rightarrow$ Vect $t_{k}$ is represented by the enveloping algebra $U \mathfrak{g}_{\mathfrak{b}}$ of the Drinfeld double of $\mathfrak{b}$. The object $U \mathfrak{g}_{\mathfrak{b}}$ with its standard coproduct is not a coalgebra in the deformed category $\mathrm{DY}_{\mathfrak{b}}^{\Phi}$ due to the non-triviality of the associativity constraints. However, the polarization $U \mathfrak{g}_{\mathfrak{b}} \simeq M_{-} \otimes M_{+}$, where $M_{ \pm}$are the Verma modules $\operatorname{Ind}_{\mathfrak{b}_{\mp}}^{\mathfrak{g}_{\mathfrak{b}}}$, with $\mathfrak{b}_{-}=\mathfrak{b}$ and $\mathfrak{b}_{+}=\mathfrak{b}^{*}$, realizes $U \mathfrak{g}_{\mathfrak{b}}$ as the tensor product of two coalgebra objects.

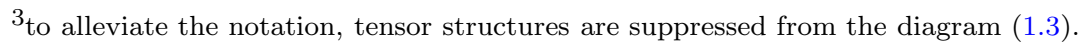

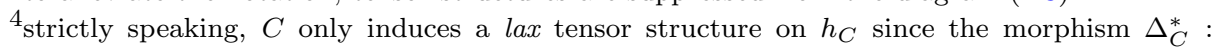
$h_{C}(U) \otimes h_{C}(V) \rightarrow h_{C}(U \otimes V)$ induced by the coproduct of $C$ may not be invertible. We shall ignore this point, since all lax tensor structures we shall encounter are easily seen to be invertible.
} 
This yields a tensor structure on the functor $F_{\mathfrak{b}}=h_{M_{-} \otimes M_{+}}: \mathrm{DY} \mathfrak{b}_{\mathfrak{b}}^{\Phi} \rightarrow$ Vect $t_{\mathrm{k}}$, and therefore on the forgetful functor $h_{U \mathfrak{g}_{\mathfrak{b}}}$.

1.9. Our starting point is to consider the restriction $\operatorname{Res}_{\mathfrak{a}, \mathfrak{b}}: \mathrm{DY}_{\mathfrak{b}} \rightarrow \mathrm{DY}_{\mathfrak{a}}$ corresponding to a split pair of finite-dimensional Lie bialgebras $\mathfrak{a} \hookrightarrow \mathfrak{b}$ as a relative forgetful functor, which is represented by the $\left(\mathfrak{g}_{\mathfrak{b}}, \mathfrak{g}_{\mathfrak{a}}\right)$-bimodule $U \mathfrak{g}_{\mathfrak{b}}$. We then factorise $U \mathfrak{g}_{\mathfrak{b}}$ as the tensor product of two coalgebra objects $L_{-}, N_{+}$in the braided tensor category of $\left(\mathfrak{g}_{\mathfrak{b}}, \mathfrak{g}_{\mathfrak{a}}\right)$-bimodules, with associativity constraints given by $\Phi_{\mathfrak{b}} \cdot \Phi_{\mathfrak{a}}^{-1}$. Just as the Verma modules $M_{-}, M_{+}$are related to the decomposition $\mathfrak{g}_{\mathfrak{b}}=\mathfrak{b}_{-} \oplus \mathfrak{b}_{+}$, $L_{-}$and $N_{+}$correspond to the asymmetric decomposition

$$
\mathfrak{g}_{\mathfrak{b}}=\mathfrak{m}_{-} \oplus \mathfrak{p}_{+}
$$

where $\mathfrak{m}_{-}=\operatorname{Ker}(p) \subset \mathfrak{b}_{-}$and $\mathfrak{p}_{+}=i\left(\mathfrak{a}_{-}\right) \oplus \mathfrak{b}_{+}$. The factorisation $U \mathfrak{g}_{\mathfrak{b}} \cong L_{-} \otimes N_{+}$ induces a tensor structure on the functor $h_{L_{-} \otimes N_{+}}: \mathrm{DY}_{\mathfrak{b}}^{\Phi} \rightarrow \mathrm{DY}_{\mathfrak{a}}^{\Phi}$, and therefore one on $\operatorname{Res}_{\mathfrak{a}, \mathfrak{b}} \cong h_{L_{-} \otimes N_{+}}$.

As in [14, Part II], this tensor structure can also be defined when $\mathfrak{a}$ or $\mathfrak{b}$ are infinite-dimensional. This amounts to replacing the Verma module $N_{+}$, which is not equicontinuous if $\mathfrak{b}$ is infinite-dimensional, with its appropriately topologised continuous dual $N_{+}^{\vee}$, and the Yoneda functor $h_{L_{-} \otimes N_{+}}$with $\operatorname{Hom}_{\mathfrak{g}_{\mathfrak{b}}}\left(L_{-}, N_{+}^{\vee} \otimes V\right)$.

Having constructed the tensor structure $J_{\mathfrak{a}, \mathfrak{b}}$, the existence of the natural transformation $u_{\mathfrak{a}, \mathfrak{b}, \mathfrak{c}}$ satisfying the associativity constraint (1.1) is readily obtained from that of the natural transformations $v_{\mathfrak{a}, \mathfrak{b}}, v_{\mathfrak{b}, \mathfrak{c}}, v_{\mathfrak{a}, \mathfrak{c}}$ of Theorem 1.7 by requiring the commutativity of the diagram (1.3), and using the fact that the functor $\widetilde{F}_{\mathfrak{a}}$ is an equivalence. It seems an interesting problem to give an intrinsic construction of $u_{\mathfrak{a}, \mathfrak{b}, \mathfrak{c}}$ which does not rely on Etingof-Kazhdan quantisation.

1.10. We now sketch the construction of a natural transformation $v_{\mathfrak{a}, \mathfrak{b}}$ which makes the diagram (1.2) commute, assuming again that $\mathfrak{a}, \mathfrak{b}$ are finite-dimensional.

As pointed out to us by Pavel Etingof, just as $\left(\operatorname{Res}_{\mathfrak{a}, \mathfrak{b}}, J_{\mathfrak{a}, \mathfrak{b}}\right)$ may be replaced by the isomorphic Yoneda functor $\left(h_{L_{-} \otimes N_{+}}, \Delta_{L_{-} \otimes N_{+}}^{*}\right)$, the tensor restriction functor $\left(\operatorname{Res}_{U_{\hbar} \mathfrak{a}, U_{\hbar} \mathfrak{b}}\right.$, id) can be replaced by $\left(h_{L_{-}^{\hbar} \otimes N_{+}^{\hbar}}\right.$, id), where $L_{-}^{\hbar}, N_{+}^{\hbar}$ are quantum analogues of the modules $L_{-}, N_{+}$. This reduces the problem to proving the commutativity of

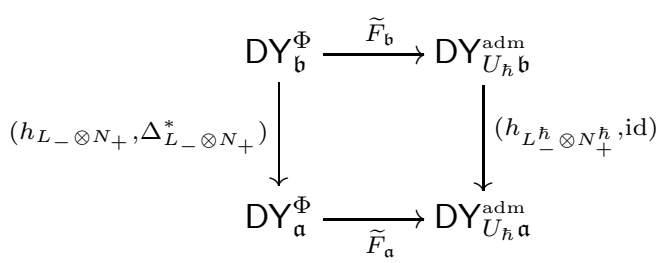

1.11. The commutativity of (1.4) amounts to proving the isomorphisms

$$
\widetilde{F}_{\mathfrak{a}} \circ \widetilde{F}_{\mathfrak{b}}\left(L_{-}\right) \simeq L_{-}^{\hbar} \quad \text { and } \quad \widetilde{F}_{\mathfrak{a}} \circ \widetilde{F}_{\mathfrak{b}}\left(N_{+}\right) \simeq N_{+}^{\hbar}
$$

as coalgebras in the category of $\left(U_{\hbar} \mathfrak{g}_{\mathfrak{b}}, U_{\hbar} \mathfrak{g}_{\mathfrak{a}}\right)$-bimodules. When $\mathfrak{a}=0$, the Verma modules $L_{-}, N_{+}$coincide with $M_{-}, M_{+}$, and it is easy to construct an isomorphism between $\widetilde{F}_{\mathfrak{b}}\left(M_{ \pm}\right)$and the quantum counterparts $M_{ \pm}^{\hbar}$ of $M_{ \pm}$. In general, however, the proof of (1.5) is more involved, and relies on the functoriality of the Drinfeld-Yetter modules $L_{-}, N_{+}$, and of their quantisation via $\widetilde{F}_{\mathfrak{a}} \circ \widetilde{F}_{\mathfrak{b}}$, with respect to morphisms of split pairs of Lie bialgebras. The latter is obtained from the 
description of the Etingof-Kazhdan quantisation functor in terms of PROPs [15], and the realization of $L_{-}, N_{+}$as universal objects in a (new) colored PROP which describes split inclusions of Lie bialgebras.

1.12. Our interest in Theorems 1.6 and 1.7 comes from quasi-Coxeter quasitriangular quasibialgebras [29] and their categorical counterparts, braided quasi-Coxeter categories [2]. If $(W, S)$ is a Coxeter group with Coxeter graph $D$, a braided quasiCoxeter category of type $W$ consists of 1 ) a family of braided tensor categories $\mathcal{Q}_{B}$ labelled by the subgraphs $B$ of $D, 2$ ) a tensor restriction functor $F_{B^{\prime} B}: \mathcal{Q}_{B} \rightarrow \mathcal{Q}_{B^{\prime}}$ for any inclusion $B^{\prime} \subseteq B$, and 3) an automorphism $S_{i}^{\mathcal{Q}}$ of the restriction functor $F_{\emptyset i}: \mathcal{Q}_{\{i\}} \rightarrow \mathcal{Q}_{\emptyset}$, called local monodromy, for any vertex $i$ of $D$. These data satisfy various compatibilities which guarantee in particular that the generalised braid group $B_{W}$ corresponding to $W$ acts on the restriction functor $F_{\emptyset D}: \mathcal{Q}_{D} \rightarrow \mathcal{Q}_{\emptyset}$.

Such a structure arises in particular from a quantum Kac-Moody algebra $U_{\hbar} \mathfrak{g}$ with Weyl group $W$ [2]. The diagrammatic categories $\mathcal{Q}_{B}$ are the integrable, highest weight categories of the standard Levi subalgebras of $U_{\hbar} \mathfrak{g}$ with braiding given by their $R$-matrices, the $F_{B^{\prime} B}$ are the standard restriction functors, and the corresponding braid group representations the quantum Weyl group representations.

We prove in [2] that this structure can be transferred to the underformed enveloping algebra $U \mathfrak{g} \llbracket \hbar \rrbracket$, and put in a given normal form. Specifically, the braided quasi-Coxeter category structure arising from $U_{\hbar} \mathfrak{g}$ is equivalent to one where the diagrammatic categories are the integrable, highest weight categories of the standard Levi subalgebras of $U \mathfrak{g} \llbracket \hbar \rrbracket$, with commutativity and associativity constraints deformed by the associator $\Phi$, and the $F_{B^{\prime} B}$ are the standard restriction functors endowed with appropriate tensor structures. The horizontal transport of structure is given by the collection of Etingof-Kazhdan equivalences corresponding to the Levi subalgebras of $\mathfrak{g}$, while the vertical matching of the quantum and classical restriction functors relies on Theorems 1.6 and 1.7.

We show in [3] that braided quasi-Coxeter category arising from $U \mathfrak{g} \llbracket \hbar \rrbracket$ which have the same normal form as those transferred from $U_{\hbar} \mathfrak{g}$ are rigid. We then use this in [4] to prove that the monodromy of the rational Casimir connection of a symmetrisable Kac-Moody algebra is described by the quantum Weyl group operators of $U_{\hbar} \mathfrak{g}$, thus extending a result of the second author valid when $\mathfrak{g}$ is finite-dimensional $[29,30]$.

1.13. Outline of the paper. In Section 2, we review the construction of the Etingof-Kazhdan fiber functor, quantisation, and Tannakian equivalence. In Section 3, we generalise the first one by introducing the generalised Verma modules $L_{-}, N_{+}$, and obtain a relative fiber functor $F_{\mathfrak{a}, \mathfrak{b}}: \mathrm{DY}_{\mathfrak{b}}^{\Phi} \rightarrow \mathrm{DY}_{\mathfrak{a}}^{\Phi}$. In Section 4 , we define the quantum Verma modules $L_{-}^{\hbar}$ and $N_{+}^{\hbar}$, use them to define a quantum fiber functor $F_{\mathfrak{a}, \mathfrak{b}}^{\hbar}: \mathrm{DY}_{U_{\hbar} \mathfrak{b}}^{\mathrm{adm}} \rightarrow \mathrm{DY}_{U_{\hbar} \mathfrak{a}}^{\mathrm{adm}}$, and construct an isomorphism $v_{\mathfrak{a}, \mathfrak{b}}: \widetilde{F}_{\mathfrak{a}} \circ F_{\mathfrak{a}, \mathfrak{b}} \simeq F_{\mathfrak{a}, \mathfrak{b}}^{\hbar} \circ \widetilde{F}_{\mathfrak{b}}$ assuming the quantisation isomorphisms (1.5). In Section 5, we generalise the Etingof-Kazhdan quantisation to the relative case, using the functor $F_{\mathfrak{a}, \mathfrak{b}}$ to construct a Hopf algebra object in $D Y_{\mathfrak{a}}^{\Phi}$ and obtain an alternative quantisation of $\mathfrak{b}$ via the Radford biproduct. In Section 6, we review the description of Etingof-Kazhdan quantisation by PROPs, and use it to give an alternative proof that the Tannakian functor $\widetilde{F}_{\mathfrak{b}}$ is an equivalence. In Section 7 , we show that the quantum Verma modules $L_{-}^{\hbar}$ and $N_{+}^{\hbar}$ are isomorphic to the Etingof-Kazhdan quantisation of their classical counterparts $L_{-}, N_{+}$, thereby proving (1.5), by using 
a suitably defined colored PROP which describes split pairs of Lie bialgebras. We also show that the results of Sections 3, 4, and 5 can be lifted to this PROP, thus establishing their functoriality with respect to morphisms of split inclusions of Lie bialgebras. In Section 8, we review Ševera's alternative construction of a quantisation functor for Lie bialgebras [27], and use it to obtain a stronger functoriality of the tensor functor $F_{\mathfrak{a}, \mathfrak{b}}$. Finally, in Appendix A, we review Majid's description of the quantum double as a double crossed product of Hopf algebras, and describe the braided tensor equivalence between modules over a quantum double and DrinfeldYetter modules.

1.14. Acknowledgments. The main results of this paper first appeared in more condensed form in the preprint [1]. The latter is superseded by the present paper, and its companion [2]. We are very grateful to Pavel Etingof for his continuing interest throughout this project, and in particular for correspondence and several enlightening discussions on foundational and other aspects of quantisation.

\section{ETINGOF-KAZHDAN QUANTISATION}

With the exception of Sections 2.18-2.23, which contain a detailed discussion of admissible Drinfeld-Yetter modules over a quantised universal enveloping algebra, and 2.25 , this section follows [14, Part II] and [16, §4] closely.

2.1. Topological vector spaces. Let $\mathrm{k}$ be a field of characteristic zero endowed with the discrete topology, and $V$ a topological vector space over k. The topology on $V$ is linear if the open subspaces in $V$ form a basis of neighborhoods of zero.

Let $V$ be endowed with a linear topology, and $p$ the natural map

$$
p: V \longrightarrow \lim _{\longleftarrow} V / U
$$

where the inverse limit is taken over the open subspaces $U \subseteq V$. $V$ is called separated if $i$ is injective, and complete if $p$ is surjective. Note that

- Since an open subspace of a topological vector space is also closed, the quotient topology on each $V / U$ is the discrete one. The corresponding product topology on $\lim V / U$ is linear, with a basis of neighborhoods of zero given by the finite intersections $\bigcap p_{U}^{-1}(0)$, where $p_{U}: \lim V / U^{\prime} \rightarrow V / U$ are the projection maps. Moreover, $\lim V / U$ is separated and complete.

- The map $p$ is continuous. It need not be open in general, but it is if $p$ is surjective. It follows that if $V$ is separated and complete, $p$ is a homeomorphism.

Throughout this paper, we shall call topological vector space a linear, complete, separated topological vector space. Note that a finite-dimensional topological vector space is necessarily endowed with the discrete topology.

2.2. If $V, W$ are topological vector spaces, we let $\operatorname{Hom}_{\mathrm{k}}(V, W)$ be the topological vector space of continuous linear maps from $V$ to $W$, equipped with the weak topology. Namely, a basis of neighborhoods of zero in $\operatorname{Hom}_{\mathrm{k}}(V, W)$ is given by the subspaces

$$
Y\left(v_{1}, \ldots, v_{n} ; W_{1}, \ldots, W_{n}\right)=\left\{T \in \operatorname{Hom}_{\mathrm{k}}(V, W) \mid T\left(v_{i}\right) \in W_{i}, i=1, \ldots, n\right\}
$$

where $n \in \mathbb{N}, v_{i} \in V$ and $W_{i}$ are open subspaces in $W$ for all $i=1, \ldots, n$. 
In particular, if $W=\mathrm{k}$ with the discrete topology, the space $V^{*}=\operatorname{Hom}_{\mathrm{k}}(V, \mathrm{k})$ has a basis of neighborhoods of zero given by orthogonal complements of finitedimensional subspaces in $V$. When $V$ is finite-dimensional, $V^{*}$ is the full linear dual of $V$, and the weak topology the discrete topology.

2.3. Given two topological vector spaces $V$ and $W$, define their topological tensor product as

$$
V \widehat{\otimes} W=\lim V / V^{\prime} \otimes W / W^{\prime}=\lim V \otimes W /\left(V^{\prime} \otimes W+V \otimes W^{\prime}\right)
$$

where the limit is take over open subspaces of $V$ and $W$, and given the product topology. Then, $V \widehat{\otimes} W$ is a topological vector space, and the tensor product $\widehat{\otimes}$ endows the category Vect $k_{k}$ of topological vector spaces over $k$ with the structure of a symmetric monoidal category with internal Hom's. Moreover, the duality functor satisfies $(V \widehat{\otimes} W)^{*} \cong V^{*} \widehat{\otimes} W^{*}$, and is therefore a contravariant tensor endofunctor of Vect $\mathrm{k}_{\mathrm{k}}$.

2.4. Formal power series. Let $\hbar$ be a formal variable, and endow $K=k \llbracket \hbar \rrbracket$ with the $\hbar$-adic topology given by the subspaces $\hbar^{n} \mathrm{~K}, n \geq 0$. Let $V$ be a topological vector space. The space $V \llbracket \hbar \rrbracket=V \widehat{\otimes} \mathrm{K}$ of formal power series in $\hbar$ with coefficients in $V$ is also a topological vector space with the structure of a topological K-module. A topological $\mathrm{K}$-module is topologically free if it is isomorphic to $V \llbracket \hbar \rrbracket$ for some topological vector space $V$ as $\mathrm{K}$-module.

The additive category Vect $\mathrm{K}_{\mathrm{K}}$ of topologically free $\mathrm{K}$-modules, where morphisms are continuous $\mathrm{K}$-linear maps, has a natural symmetric monoidal structure with internal Hom's. The tensor product is defined as the quotient of the tensor product $V \widehat{\otimes} W$ by the closure of the image of the operator $\hbar \otimes 1-1 \otimes \hbar$, and will be still denoted by $\widehat{\otimes}$.

There is an extension of scalar functor from the category of topological vector spaces to Vect $\mathrm{K}_{\mathrm{K}}$, mapping $V$ to $V \llbracket \hbar \rrbracket$. This functor respects the tensor product, i.e., $(V \widehat{\otimes} W) \llbracket \hbar \rrbracket$ is naturally isomorphic to $V \llbracket \hbar \rrbracket \widehat{\otimes} W \llbracket \hbar \rrbracket$.

Henceforth, unless otherwise specified, we will denote by $\otimes$ the complete tensor products of topological $\mathrm{k}$-vector spaces and topologically free $\mathrm{K}$-modules.

2.5. Lie bialgebras. A Lie bialgebra is a triple $\left(\mathfrak{b},[,]_{\mathfrak{b}}, \delta_{\mathfrak{b}}\right)$ where

- $\mathfrak{b}$ is a discrete vector space;

- $\left(\mathfrak{b},[,]_{\mathfrak{b}}\right)$ is a Lie algebra, i.e., $[,]_{\mathfrak{b}}: \mathfrak{b} \otimes \mathfrak{b} \rightarrow \mathfrak{b}$ is anti-symmetric and satisfies the Jacobi identity

$$
[,]_{\mathfrak{b}} \circ \operatorname{id}_{\mathfrak{b}} \otimes[,]_{\mathfrak{b}} \circ\left(\operatorname{id}_{\mathfrak{b} \otimes 3}+(123)+(132)\right)=0
$$

- $\left(\mathfrak{b}, \delta_{\mathfrak{b}}\right)$ is a Lie coalgebra, i.e., $\delta_{\mathfrak{b}}: \mathfrak{b} \rightarrow \mathfrak{b} \otimes \mathfrak{b}$ is anti-symmetric and satisfies the co-Jacobi identity

$$
\left(\mathrm{id}_{\mathfrak{b} \otimes 3}+(123)+(132)\right) \circ \mathrm{id}_{\mathfrak{b}} \otimes \delta_{\mathfrak{b}} \circ \delta_{\mathfrak{b}}=0 ;
$$

- the cobracket $\delta_{\mathfrak{b}}$ satisfies the cocycle condition

$$
\delta_{\mathfrak{b}} \circ[,]_{\mathfrak{b}}=\operatorname{ad}_{\mathfrak{b}} \circ \mathrm{id}_{\mathfrak{b}} \otimes \delta_{\mathfrak{b}} \circ\left(\operatorname{id}_{\mathfrak{b} \otimes 2}-(12)\right),
$$

as maps $\mathfrak{b} \otimes \mathfrak{b} \rightarrow \mathfrak{b} \otimes \mathfrak{b}$, where $\operatorname{ad}_{\mathfrak{b}}: \mathfrak{b} \otimes \mathfrak{b} \otimes \mathfrak{b} \rightarrow \mathfrak{b} \otimes \mathfrak{b}$ denotes the left adjoint action of $\mathfrak{b}$ on $\mathfrak{b} \otimes \mathfrak{b}$. 
2.6. Manin triples. A Manin triple is the data of a Lie algebra $\mathfrak{g}$ with

- a nondegenerate invariant inner product $\langle-,-\rangle$

- isotropic Lie subalgebras $\mathfrak{b}_{ \pm} \subset \mathfrak{g}$

such that

- $\mathfrak{g}=\mathfrak{b}_{-} \oplus \mathfrak{b}_{+}$as vector spaces

- the commutator of $\mathfrak{g}$ is continuous with respect to the topology obtained by putting the discrete and the weak topologies on $\mathfrak{b}_{-}$and $\mathfrak{b}_{+}$respectively.

- the inner product defines an isomorphism $\mathfrak{b}_{+} \rightarrow \mathfrak{b}_{-}^{*}$

Under these assumptions, the commutator on $\mathfrak{b}_{+} \simeq \mathfrak{b}_{-}^{*}$ induces a cobracket $\delta: \mathfrak{b}_{-} \rightarrow \mathfrak{b}_{-} \otimes \mathfrak{b}_{-}$which satisfies the cocycle condition (2.1). Therefore, $\mathfrak{b}_{-}$is canonically endowed with a Lie bialgebra structure. In general, however, $\mathfrak{b}_{+}$is only a topological Lie bialgebra.

2.7. Drinfeld double. Every Lie bialgebra $\left(\mathfrak{b},[,]_{\mathfrak{b}}, \delta_{\mathfrak{b}}\right)$ gives rise to a Manin triple. The Drinfeld double of $\mathfrak{b}$ is the Lie algebra $\mathfrak{g}_{\mathfrak{b}}$ defined as follows.

As a vector space, $\mathfrak{g}_{\mathfrak{b}}=\mathfrak{b} \oplus \mathfrak{b}^{*}$. The pairing $\langle-,-\rangle: \mathfrak{b} \otimes \mathfrak{b}^{*} \rightarrow \mathrm{k}$ extends uniquely to a symmetric non-degenerate bilinear form on $\mathfrak{g}_{\mathfrak{b}}$, with respect to which $\mathfrak{b}, \mathfrak{b}^{*}$ are isotropic. The Lie bracket on $\mathfrak{g}_{\mathfrak{b}}$ is defined as the unique bracket which coincides with $[,]_{\mathfrak{b}}$ on $\mathfrak{b}$, with $\delta_{\mathfrak{b}}^{t}$ on $\mathfrak{b}^{*}$, and is compatible with $\langle-,-\rangle$, i.e., satisfies

$$
\langle[x, y], z\rangle=\langle x,[y, z]\rangle
$$

for all $x, y, z \in \mathfrak{g}_{\mathfrak{b}}$. The mixed bracket of $x \in \mathfrak{b}$ and $\phi \in \mathfrak{b}^{*}$ is then given by

$$
[x, \phi]=\operatorname{ad}^{*}(x)(\phi)-\operatorname{ad}^{*}(\phi)(x)
$$

where ad* is the coadjoint actions of $\mathfrak{b}$ on $\mathfrak{b}^{*}$ and of $\mathfrak{b}^{*}$ on $\left(\mathfrak{b}^{*}\right)^{* 5}$.

2.8. Drinfeld-Yetter modules. A Drinfeld-Yetter module over a Lie bialgebra $\mathfrak{b}$ is a triple $\left(V, \pi, \pi^{*}\right)$, where $\pi: \mathfrak{b} \otimes V \rightarrow V$ gives $V$ the structure of a left $\mathfrak{b}$-module, that is

$$
\pi \circ[,] \otimes \mathrm{id}=\pi \circ(\mathrm{id} \otimes \pi)-\pi \circ(\mathrm{id} \otimes \pi) \circ(21)
$$

as maps $\mathfrak{b} \otimes \mathfrak{b} \otimes V \rightarrow V, \pi^{*}: V \rightarrow \mathfrak{b} \otimes V$ gives $V$ the structure of a right $\mathfrak{b}$-comodule, that is

$$
\delta \otimes \mathrm{id} \circ \pi^{*}=(21) \circ\left(\mathrm{id} \otimes \pi^{*}\right) \circ \pi^{*}-\left(\mathrm{id} \otimes \pi^{*}\right) \circ \pi^{*}
$$

as maps $V \rightarrow \mathfrak{b} \otimes \mathfrak{b} \otimes V$, and the maps $\pi, \pi^{*}$ satisfy the following compatibility condition in $\operatorname{End}(\mathfrak{b} \otimes V)$

$$
\pi^{*} \circ \pi-\operatorname{id} \otimes \pi \circ(12) \circ \text { id } \otimes \pi^{*}=[,] \otimes \text { id } \circ \text { id } \otimes \pi^{*}-\operatorname{id} \otimes \pi \circ \delta \otimes \text { id . }
$$

A Drinfeld-Yetter module $V$ gives rise to an action of the Drinfeld double $\mathfrak{g}_{\mathfrak{b}}$ on $V$, with $\phi \in \mathfrak{b}^{*}$ acting by $\phi \otimes \mathrm{id} \circ \pi^{*}$. The corresponding map $\mathfrak{g}_{\mathfrak{b}} \rightarrow \operatorname{End}_{\mathfrak{k}}(V)$ is easily seen to be continuous if $\operatorname{End}_{\mathfrak{k}}(V)$ is given the weak topology, and $\mathfrak{g}_{\mathfrak{b}}$ the product of the discrete and weak topologies on $\mathfrak{b}, \mathfrak{b}^{*}$, as in 2.6. Conversely, a continuous Lie algebra homomorphism $\mathfrak{g}_{\mathfrak{b}} \rightarrow \operatorname{End}_{\mathfrak{k}}(V)$ gives in particular rise to a locally finite action of $\mathfrak{b}^{*}$, and therefore to a Drinfeld-Yetter module on $V$, with $\pi^{*}(v)=\sum_{i} b_{i} \otimes b^{i} v$, where $\left\{b_{i}\right\},\left\{b^{i}\right\}$ are dual bases of $\mathfrak{b}, \mathfrak{b}^{*}$.

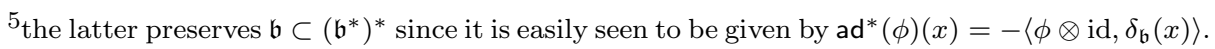


2.9. Equicontinuous modules. Let $\mathfrak{g}$ be a topological Lie algebra, and $V$ a topological vector space. $V$ is an equicontinuous $\mathfrak{g}$-module if it is endowed with a Lie algebra homomorphism $\pi_{V}: \mathfrak{g} \rightarrow \operatorname{End}_{\mathfrak{k}}(V)$ such that

- $\pi_{V}$ is continuous

- $\left\{\pi_{V}(X)\right\}_{X \in \mathfrak{g}}$ is an equicontinuous family of linear operators, i.e., for any open subspace $U \subseteq V$, there exists $U^{\prime}$ such that $\pi_{V}(X) U^{\prime} \subset U$ for all $X \in \mathfrak{g}$.

The category $\mathcal{E}_{\mathfrak{g}}$ of equicontinuous $\mathfrak{g}$-modules is a symmetric monoidal category, with respect to the completed tensor product of topological vector spaces and braiding defined by the permutation of components.

2.10. Topological Drinfeld-Yetter modules. The notion of Drinfeld-Yetter module can be formulated in any symmetric tensor category, in particular that of topological vector spaces, with completed tensor product and continuous linear maps. The corresponding category $\mathrm{DY}_{\mathfrak{b}}$ is then equivalent to that of equicontinuous modules $\mathcal{E}_{\mathfrak{g}_{\mathfrak{b}}}$ over $\mathfrak{g}_{\mathfrak{b}}$ defined in $\S 2.9$.

2.11. $r$-matrix. If $U, V \in \mathrm{DY}_{\mathfrak{b}}$, define $r_{U, V} \in \operatorname{End}_{\mathfrak{k}}(U \otimes V)$ as the composition

$$
r_{U V}=\pi_{U} \otimes \mathrm{id} \circ(12) \circ \mathrm{id} \otimes \pi_{V}^{*} .
$$

Then, $r$ satisfies the classical Yang-Baxter equations on $U \otimes V \otimes W$

$$
\left[r_{U V}, r_{U W}\right]+\left[r_{U V}, r_{V W}\right]+\left[r_{U W}, r_{V W}\right]=0
$$

and is such that $\Omega_{U V}=r_{U V}+r_{V U}^{21}$ is a morphism of Drinfeld-Yetter modules.

Under the identification $\mathrm{DY}_{\mathfrak{b}}=\mathcal{E}_{\mathfrak{g}_{\mathfrak{b}}}$, the action of $r_{U V}$ corresponds to that of the canonical element $b_{i} \otimes b^{i} \in \mathfrak{b} \otimes \mathfrak{b}^{*} \cong \operatorname{End}_{k}(\mathfrak{b}) \ni \operatorname{id}_{\mathfrak{b}}$, and that of $\Omega_{U V}$ to that of the canonical element $b_{i} \otimes b^{i}+b^{i} \otimes b_{i}$ in $\mathfrak{g}_{\mathfrak{b}} \otimes \mathfrak{g}_{\mathfrak{b}}$ representing the bilinear form $\langle-,-\rangle$.

2.12. Drinfeld category. Following Drinfeld [8], one can define a $\mathrm{K}$-linear deformation $D Y_{\mathfrak{b}}^{\Phi}=\mathcal{E}_{\mathfrak{g}_{\mathfrak{b}}}^{\Phi}$ of $D Y_{\mathfrak{b}}=\mathcal{E}_{\mathfrak{g}_{\mathfrak{b}}}$ as a braided monoidal category using an associator $\Phi$ as follows. The objects of $D Y_{\mathfrak{b}}^{\Phi}$ are Drinfeld-Yetter $\mathfrak{b}-$ modules in the category of topologically free $\mathrm{k} \llbracket \hbar \rrbracket-$ modules with commutativity and associativity constraints given respectively by

$$
\beta_{U V}=(12) \circ \exp \left(\frac{\hbar}{2} \Omega_{U V}\right) \quad \text { and } \quad \Phi_{U V W}=\Phi\left(\hbar \Omega_{U V}, \hbar \Omega_{V W}\right)
$$

2.13. The Verma modules $M_{-}$and $M_{+}$. Set $\mathfrak{b}_{-}=\mathfrak{b}, \mathfrak{b}_{+}=\mathfrak{b}^{*}$, and consider the $\mathfrak{g}_{\mathfrak{b}}$-modules $M_{-}, M_{+}$given by

$$
M_{-}=\operatorname{Ind}_{\mathfrak{b}_{+}}^{\mathfrak{g}_{\mathfrak{b}}} \mathrm{k} \quad \text { and } \quad M_{+}=\operatorname{Ind}_{\mathfrak{b}_{-}}^{\mathfrak{g}_{\mathfrak{b}}} \mathrm{k} .
$$

The modules $M_{-}$and $M_{+}^{\vee}$, the dual of $M_{+}$with appropriate topology, are equicontinuous $\mathfrak{g}_{\mathfrak{b}}$-modules.

The module $M_{-}$is an equicontinuous $\mathfrak{g}_{\mathfrak{b}}$-module with respect to the discrete topology. The topology on $M_{+}$comes, instead, from the identification of vector spaces

$$
M_{+} \simeq U \mathfrak{b}_{+}=\bigcup_{n \geq 0}\left(U \mathfrak{b}_{+}\right)_{n}
$$


where $\left(U \mathfrak{b}_{+}\right)_{n}$ is the set of elements of degree at most $n$. The topology on $\left(U \mathfrak{b}_{+}\right)_{n}$ is defined through the linear isomorphism

$$
\xi_{n}: \bigoplus_{j=0}^{n} S^{j} \mathfrak{b}_{+} \rightarrow\left(U \mathfrak{b}_{+}\right)_{n}
$$

where $S^{j} \mathfrak{b}_{+}$is considered as a topological subspace of $\left(\mathfrak{b}_{-}^{\otimes j}\right)^{*}$, embedded with the weak topology. Finally, $U \mathfrak{b}_{+}$is equipped with the topology of the colimit. Namely, a set $U \subseteq U \mathfrak{b}_{+}$is open if and only if $U \cap\left(U \mathfrak{b}_{+}\right)_{n}$ is open for all $n$. With respect to the topology just described, the action of $\mathfrak{g}_{\mathfrak{b}}$ on $M_{+}$is continuous.

Consider now the vector space of continuous linear functionals on $M_{+}$

$$
M_{+}^{*}=\operatorname{Hom}_{\mathrm{k}}\left(M_{+}, \mathrm{k}\right) \simeq \lim \operatorname{Hom}_{\mathrm{k}}\left(\left(U \mathfrak{b}_{+}\right)_{n}, \mathrm{k}\right) .
$$

It is natural to put the discrete topology on $\left(U \mathfrak{b}_{+}\right)_{n}^{*}$, since, as a vector space,

$$
\left(U \mathfrak{b}_{+}\right)_{n}^{*} \simeq \bigoplus_{j=0}^{n} S^{j} \mathfrak{b}_{+}^{*} \simeq \bigoplus_{j=0}^{n} S^{j} \mathfrak{b}_{-} \simeq\left(U \mathfrak{b}_{-}\right)_{n}
$$

We then consider on $M_{+}^{*}$ the topology of the limit and denote the resulting topological space by $M_{+}^{\vee}$. This defines, in particular, a filtration by subspaces $\left(M_{+}^{\vee}\right)_{n}$ satisfying

$$
0 \rightarrow\left(M_{+}^{\vee}\right)_{n} \rightarrow M_{+}^{\vee} \rightarrow U\left(\mathfrak{b}_{+}\right)_{n}^{*} \rightarrow 0
$$

and such that $M_{+}^{\vee}=\lim M_{+}^{\vee} /\left(M_{+}^{\vee}\right)_{n}$. The topology of the limit on $M_{+}^{*}$ is, in general, stronger than the weak topology of the dual. Since the action of $\mathfrak{g}_{\mathfrak{b}}$ on $M_{+}$ is continuous, $M_{+}^{\vee}$ has a natural structure of $\mathfrak{g}_{\mathfrak{b}}$-module. In particular, this is an equicontinuous $\mathfrak{g}_{\mathfrak{b}}$-action.

2.14. Properties of Verma modules. The modules $M_{ \pm}$are identified, as $\mathfrak{b}_{ \pm^{-}}$ modules, with the enveloping universal algebras $U \mathfrak{b}_{ \pm}$. The comultiplication on the latter induce the $U \mathfrak{g}_{\mathfrak{b}}$-intertwiners $i_{ \pm}: M_{ \pm} \rightarrow M_{ \pm} \otimes M_{ \pm}$, mapping the vectors $1_{ \pm}$ to the $\mathfrak{b}_{\mp}$-invariant vectors $1_{ \pm} \otimes 1_{ \pm}$.

For any $f, g \in M_{+}^{\vee}$, consider the linear functional $M_{+} \rightarrow \mathrm{k}$ defined by $v \mapsto$ $(f \otimes g)\left(i_{+}(v)\right)$. This defines a morphism of modules $i_{+}^{*}: M_{+}^{\vee} \otimes M_{+}^{\vee} \rightarrow M_{+}^{\vee}$. The pairs $\left(M_{-}, i_{-}\right)$and $\left(M_{+}^{\vee}, i_{+}^{*}\right)$ form, respectively, a coalgebra and an algebra object in $\mathcal{E}_{\mathfrak{g}_{\mathfrak{b}}}^{\Phi}$.

For any $V \in \mathcal{E}_{\mathfrak{g}_{\mathfrak{b}}}^{\Phi}$, the vector space $\operatorname{Hom}_{\mathfrak{g}_{\mathfrak{b}}}\left(M_{-}, M_{+}^{\vee} \otimes V\right)$ is naturally isomorphic to $V$, as topological vector space, through the isomorphism $f \mapsto\left(1_{+} \otimes 1\right) F_{\mathfrak{b}}\left(1_{-}\right)$.

2.15. The fiber functor. Let $F_{\mathfrak{b}}: \mathcal{E}_{\mathfrak{g}_{\mathfrak{b}}}^{\Phi} \rightarrow \mathrm{Vect}_{\mathrm{K}}$ be the functor given by

$$
F_{\mathfrak{b}}(V)=\operatorname{Hom}_{\mathcal{E}_{\mathfrak{q}_{\mathfrak{b}}}^{\Phi}}\left(M_{-}, M_{+}^{\vee} \otimes V\right) .
$$

Define a natural transformation $J_{V, W}: F_{\mathfrak{b}}(V) \otimes F_{\mathfrak{b}}(W) \rightarrow F_{\mathfrak{b}}(V \otimes W)$ by

$$
J_{V, W}(v \otimes w)=\left(i_{+}^{\vee} \otimes \mathrm{id}^{\otimes 2}\right) \circ A^{-1} \circ \beta_{23}^{-1} \circ A \circ(v \otimes w) \circ i_{-}
$$

where $A$ is the isomorphism

$$
\left(V_{1} \otimes V_{2}\right) \otimes\left(V_{3} \otimes V_{4}\right) \rightarrow V_{1} \otimes\left(\left(V_{2} \otimes V_{3}\right) \otimes V_{4}\right)
$$

defined by the action of $\left(1 \otimes \Phi_{2,3,4}\right) \Phi_{1,2,34}$. 
Theorem. [14] The natural transformation $J$ is invertible, and defines a tensor structure on the functor $F_{\mathfrak{b}}$, that is satisfies

$$
F_{\mathfrak{b}}\left(\Phi_{U, V, W}\right) J_{U \otimes V, W} J_{U, V} \otimes \mathrm{id}=J_{U, V \otimes W} \mathrm{id} \otimes J_{V, W}
$$

as morphisms $\left(F_{\mathfrak{b}}(U) \otimes F_{\mathfrak{b}}(V)\right) \otimes F_{\mathfrak{b}}(W) \rightarrow F_{\mathfrak{b}}(U \otimes(V \otimes W))$, for any $U, V, W \in \mathcal{E}_{\mathfrak{g}_{\mathfrak{b}}}^{\Phi}$.

The algebra of endomorphisms of $F_{\mathfrak{b}}$ is therefore naturally endowed with a topological bialgebra structure. ${ }^{6}$

2.16. Etingof-Kazhdan quantisation. Let $V \in \mathcal{E}_{\mathfrak{g}_{\mathfrak{b}}}^{\Phi}$, and consider the linear map $m_{V}: F_{\mathfrak{b}}\left(M_{-}\right) \otimes F_{\mathfrak{b}}(V) \rightarrow F_{\mathfrak{b}}(V)$ given by

$$
m_{V}(x) v=\left(i_{+}^{\vee} \otimes 1\right) \Phi^{-1}(1 \otimes v) x .
$$

The map $m_{V}$ satisfies the associativity relation

$$
m_{V} \circ\left(m_{M_{-}} \otimes \mathrm{id}_{V}\right)=m_{V} \circ\left(\operatorname{id}_{M_{-}} \otimes m_{V}\right)
$$

as morphisms $F_{\mathfrak{b}}\left(M_{-}\right) \otimes F_{\mathfrak{b}}\left(M_{-}\right) \otimes F_{\mathfrak{b}}(V) \rightarrow F_{\mathfrak{b}}(V)$, and the unit condition $m_{V}\left(u_{-}\right)=$ $\operatorname{id}_{V}$, where $u_{-} \in F_{\mathfrak{b}}\left(M_{-}\right)$is the element mapping $1_{-} \in M_{-}$to $1_{+}^{*} \otimes 1_{-} \in M_{+}^{*} \otimes M_{-}$.

As a consequence, $F_{\mathfrak{b}}\left(M_{-}\right)$is an associative algebra with unit $u$ and multiplication $m_{M_{-}}$, which acts on the functor $F_{\mathfrak{b}}$. The corresponding map $F_{\mathfrak{b}}\left(M_{-}\right) \rightarrow$ $\operatorname{End}\left(F_{\mathfrak{b}}\right)$ is an embedding since $F_{\mathfrak{b}}\left(M_{-}\right)$is unital and acts on itself, and we shall identify $F_{\mathfrak{b}}\left(M_{-}\right)$with its image in $\operatorname{End}\left(F_{\mathfrak{b}}\right)$.

The (topological) coproduct on $\operatorname{End}\left(F_{\mathfrak{b}}\right)$ maps $F_{\mathfrak{b}}\left(M_{-}\right)$to $F\left(M_{-}\right) \otimes F_{\mathfrak{b}}\left(M_{-}\right)$, and coincides with the coproduct induced by the coalgebra structure on $M_{-}$given by $\Delta=J_{M_{-}, M_{-}}^{-1} \circ F_{\mathfrak{b}}\left(i_{-}\right)$. It follows that

$$
U_{\hbar} \mathfrak{b}=\left(F_{\mathfrak{b}}\left(M_{-}\right), m_{M_{-}}, u_{-}, J_{M_{-}, M_{-}}^{-1} \circ F_{\mathfrak{b}}\left(i_{-}\right), m_{\mathfrak{k}}\right)
$$

is a bialgebra, with counit $m_{\mathrm{k}}: F_{\mathfrak{b}}\left(M_{-}\right) \rightarrow \mathrm{K}$, endowed with an identification of $\mathrm{K}$-modules $F_{\mathfrak{b}}\left(M_{-}\right) \cong M_{-} \llbracket \hbar \rrbracket \cong U \mathfrak{b} \llbracket \hbar \rrbracket$.

Theorem. [14, 15]

(i) $U_{\hbar} \mathfrak{b}$ is a Hopf algebra, which is a quantisation of the Lie bialgebra $\mathfrak{b}$.

(ii) The assignment $\mathfrak{b} \mapsto U_{\hbar} \mathfrak{b}$ is functorial in the Lie bialgebra $\mathfrak{b}$.

Remark. The fact that $U_{\hbar} \mathfrak{b}$ admits an antipode follows because it is a deformation of $U \mathfrak{b}$ as a bialgebra, and the latter has an antipode. A formula for the antipode is given explicitly in [11, Prop. 4.2], and one has

$$
S=\sum_{n \geq 0}(-1)^{n} m^{(n)}(\mathrm{id}-\iota \circ \epsilon)^{\otimes n} \Delta^{(n)} .
$$

2.17. Drinfeld-Yetter modules over a Hopf algebra. If $(B, m, \imath, \Delta, \epsilon, S)$ is a Hopf algebra, a Drinfeld-Yetter module over $B$ is a triple $\left(V, \pi, \pi^{*}\right)$, where $\pi$ : $B \otimes V \rightarrow V$ gives $V$ the structure of a left $B$-module, that is

$$
\pi \circ m \otimes \mathrm{id}_{V}=\pi \circ \mathrm{id}_{B} \otimes \pi \quad \text { and } \quad \pi \circ \imath \otimes \mathrm{id}_{V}=\mathrm{id}_{V}
$$

as maps $B \otimes B \otimes V \rightarrow V$ and $V \rightarrow V$ respectively, $\pi^{*}: V \rightarrow \mathfrak{b} \otimes V$ gives $V$ the structure of a right $B$-comodule, that is

$$
\Delta^{21} \otimes \operatorname{id}_{V} \circ \pi^{*}=\operatorname{id}_{B} \otimes \pi^{*} \circ \pi^{*} \quad \text { and } \quad \epsilon \otimes \operatorname{id}_{V} \circ \pi^{*}=\operatorname{id}_{V}
$$

\footnotetext{
${ }^{6}$ By topological bialgebra we do not mean topological over $\mathrm{k} \llbracket \hbar \rrbracket$. We are instead referring to the fact that the algebra End $\left(F_{\mathfrak{b}}\right)$ has a natural comultiplication $\Delta:$ End $\left(F_{\mathfrak{b}}\right) \rightarrow$ End $\left(F_{\mathfrak{b}} \otimes F_{\mathfrak{b}}\right)$, and that End $\left(F_{\mathfrak{b}} \otimes F_{\mathfrak{b}}\right)$ can be interpreted as a completion of End $\left(F_{\mathfrak{b}}\right)^{\otimes 2}$.
} 
as maps $V \rightarrow B \otimes B \otimes V$ and $V \rightarrow V$ respectively, and the maps $\pi, \pi^{*}$ satisfy the following compatibility condition in $\operatorname{End}(B \otimes V)$

$$
\pi^{*} \circ \pi=m^{(3)} \otimes \pi \circ(13)(24) \circ S^{-1} \otimes \mathrm{id}^{\otimes 4} \circ \Delta^{(3)} \otimes \pi^{*}
$$

where $m^{(3)}=m \circ m \otimes$ id $: B^{\otimes 3} \rightarrow B$ and $\Delta^{(3)}=\Delta \otimes$ id $\circ \Delta: B \rightarrow B^{\otimes 3}$ are the iterated multiplication and comultiplication respectively. The category $\mathrm{DY}_{B}$ of Drinfeld-Yetter modules over $B$ has a natural structure of braided monoidal category. For any $V, W \in \mathrm{DY}_{B}$, the action and coaction on the tensor product $V \otimes W$ are defined, respectively, by

$\pi_{V \otimes W}=\pi_{V} \otimes \pi_{W} \circ(23) \circ \Delta \otimes \mathrm{id}^{\otimes 2} \quad$ and $\quad \pi_{V \otimes W}^{*}=m^{21} \otimes \mathrm{id}^{\otimes 2} \circ(23) \circ \pi_{V}^{*} \otimes \pi_{W}^{*}$.

The associativity constraints are trivial and the braiding is defined by $\beta_{V W}=$ (21) $\circ R_{V W}$, where the $R$-matrix $R_{V W} \in \operatorname{End}(V \otimes W)$ is defined by

$$
R_{V W}=\pi_{V} \otimes \mathrm{id} \circ(23) \mathrm{id} \otimes \pi_{W}^{*} .
$$

It follows from (2.5)-(2.6), and $m \circ$ id $\otimes S \otimes \Delta=\iota \circ \epsilon$, that $R_{V W}$ is an invertible endomorphism with inverse

$$
R_{V W}^{-1}=\pi_{V} \otimes \mathrm{id} \circ S \otimes \mathrm{id}^{\otimes 2} \circ(23) \mathrm{id} \otimes \pi_{W}^{*} .
$$

The braiding $\beta_{V W}$ is therefore invertible, with inverse $R_{V W}^{-1} \circ(12)$.

2.18. Drinfeld-Yetter modules and quantum double. Let $B$ be a finitedimensional Hopf algebra, and $B^{\circ}$ the Hopf algebra $B^{*}$ with opposite coproduct. The quantum double of $B$ is the unique quasitriangular Hopf algebra $(D B, R)$ such that 1) $D B=B \otimes B^{\circ}$ as vector spaces 2) $B$ and $B^{\circ}$ are Hopf subalgebras of $D B$ and 3) $R$ is the canonical element corresponding to $B \otimes B^{\circ} \subset D B \otimes D B$. The multiplication in $D B$ is given in Sweedler notation by

$$
b \otimes f \cdot b^{\prime} \otimes f^{\prime}=\left\langle S^{-1}\left(b_{1}^{\prime}\right), f_{1}\right\rangle\left\langle b_{3}^{\prime}, f_{3}\right\rangle b \cdot b_{2}^{\prime} \otimes f_{2} \cdot f
$$

where $f, f^{\prime} \in B^{\circ}, b, b^{\prime} \in B$, and $\langle-,-\rangle$ is the evaluation pairing [7, §13]. It is easy to see that there is a canonical equivalence of braided tensor categories $\operatorname{Rep} D B \simeq \mathrm{DY}_{B}$. For completeness, we include a detailed proof of this result in the Appendix (cf. §A.4). More specifically, any Drinfeld-Yetter $B$-module $V$ is acted upon by $B^{\circ}$ via

$$
B^{\circ} \otimes V \stackrel{\text { id } \otimes \pi_{V}^{*}}{\longrightarrow} B^{\circ} \otimes B \otimes V \stackrel{\text { ev } \otimes \mathrm{id}}{\longrightarrow} V .
$$

The compatibility condition between action and coaction guarantees that the actions of $B, B^{\circ}$ on $V$ give rise to an action of $D B$. Conversely, given any $D B$-module $V$, the map

$$
V \stackrel{\iota \otimes \mathrm{id}}{\longrightarrow} B \otimes V \stackrel{\text { id } \otimes \pi_{V}(R)}{\longrightarrow} B \otimes V
$$

defines a compatible coaction on $B$ on $V$. It is straightforward to check that this equivalence preserves the tensor product and braiding.

2.19. Duality for quantum enveloping algebras. We review below (one half of) Drinfeld's duality principle [7, 18], adapted to the setting of Lie bialgebras of arbitrary dimension.

Recall that a quantised enveloping algebra (QUE) is a Hopf algebra $B$ in Vect ${ }_{k}$ such that 
- $B$ is endowed with the $\hbar$-adic topology, that is $\left\{\hbar^{n} B\right\}_{n \geq 0}$ is a basis of neighborhoods of 0 . Equivalently, $B$ is isomorphic, as topological $\mathrm{K}$ module, to $B_{0} \llbracket \hbar \rrbracket$, for some discrete topological vector space $B_{0}$.

- $B / \hbar B$ is a connected, cocommutative Hopf algebra over k. Equivalently, $B / \hbar B$ is isomorphic to $U \mathfrak{b}$ for some Lie bialgebra $\left(\mathfrak{b},[,]_{\mathfrak{b}}, \delta_{\mathfrak{b}}\right)$ and, under this identification,

$$
\delta_{\mathfrak{b}}(b)=\frac{\Delta(\widetilde{b})-\Delta^{21}(\widetilde{b})}{\hbar} \bmod \hbar
$$

where $\widetilde{b} \in B$ is any lift of $b \in \mathfrak{b}$.

A quantised formal series Hopf algebra (QFSH) is a Hopf algebra $B$ in Vect ${ }_{k}$ such that

- $B$ is endowed with the $I$-adic topology, where $I=\epsilon^{-1}(\hbar \mathrm{k} \llbracket \hbar \rrbracket)$, that is $\left\{I^{n}\right\}_{n \geq 0}$ is a basis of neighborhoods of 0 .

- $B / \hbar B$ is a local, commutative Hopf algebra. Equivalently, $B / \hbar B$ is isomorphic, as Poisson Hopf algebra, to $(\widehat{S \mathfrak{b}}, m, \Delta, P)$ for some Lie bialgebra $\left(\mathfrak{b},[\cdot, \cdot]_{\mathfrak{b}}, \delta_{\mathfrak{b}}\right)$. Here, $\widehat{S \mathfrak{b}}$ is the completion of the symmetric algebra of $\mathfrak{b}$ with respect to its grading, $m$ is the commutative multiplication on $S \mathfrak{b}, \Delta$ is the coproduct obtained by formally starting from the Baker-CampbellHausdorff multiplication on $S \mathfrak{b}^{*} \cong U \mathfrak{b}^{*}$ (see $\S 6.5$ ), reversing all arrows and replacing $[\cdot, \cdot]_{\mathfrak{b}^{*}}=\delta_{b}^{t}$ by $\delta_{\mathfrak{b}}$, and the Poisson bracket $P$ is given by the Lie bracket $[\cdot, \cdot]_{\mathfrak{b}}$.

We denote the categories of quantised enveloping algebras and quantised formal series Hopf algebras over k by $\mathrm{QUE}(\mathrm{k})$ and $\operatorname{QFSH}(\mathrm{k})$ respectively. For every $B \in$ QUE(k), set

$$
B^{\prime}=\left\{b \in B \mid(\text { id }-\iota \circ \epsilon)^{\otimes n} \circ \Delta^{(n)}(b) \in \hbar^{n} B^{\otimes n} \text { for any } n \geq 0\right\}
$$

where $\Delta^{(n)}$ denotes the iterated coproduct defined inductively by $\Delta^{(0)}=\iota \circ \epsilon$, $\Delta^{(1)}=\mathrm{id}$, and

$$
\Delta^{(n+1)}=\Delta \otimes \mathrm{id}^{\otimes(n-1)} \circ \Delta^{(n)} .
$$

Then $B^{\prime}$, endowed with the topology induced by the $\hbar$-adic topology on $B$, is a Hopf subalgebra of $B$, and a quantised formal series Hopf algebra. Moreover, if $B / \hbar B$ is isomorphic to $U \mathfrak{b}$ for some Lie bialgebra $\mathfrak{b}$, then $B^{\prime} / \hbar B^{\prime}$ is isomorphic to the Poisson Hopf algebra $(\widehat{S \mathfrak{b}}, m, \Delta, P)$ described above.

If $B \in \mathrm{QUE}(\mathrm{k})$ is a quantisation of Lie bialgebra $\left(\mathfrak{b},[\cdot, \cdot]_{\mathfrak{b}}, \delta_{b}\right)$ where $\mathfrak{b}$ is finitedimensional, then $B^{\vee}=\left(B^{\prime}\right)^{*}$ is a QUE, with underlying Lie bialgebra $\left(\mathfrak{b}^{*}, \delta_{\mathfrak{b}}^{t},[\cdot, \cdot]_{\mathfrak{b}}^{t}\right)$. The tensor product $B \otimes\left(B^{\vee}\right)^{\circ}$ can be endowed with a unique quasitriangular Hopf algebra structure such that $B$ and $\left(B^{\vee}\right)^{\circ}$ are Hopf subalgebras, and is called the quantum double $D B$ of $B$. The corresponding $R$-matrix $R$ lies in the $\hbar$-adic completion of the algebraic tensor product $B^{\prime} \otimes\left(B^{\vee}\right)^{\circ} \subset D B^{\otimes 2}$.

2.20. Admissible Drinfeld-Yetter modules. The following notion is due to P. Etingof [13].

Definition. A Drinfeld-Yetter module $\mathcal{V}$ over a quantised enveloping algebra $B$ is called admissible if its coaction $\pi_{\mathcal{V}}^{*}: \mathcal{V} \rightarrow B \otimes \mathcal{V}$ factors through $B^{\prime} \otimes \mathcal{V}$. 
In analogy with the result described in $\S 2.18$, the category $\mathrm{DY}_{B}^{\mathrm{adm}}$ of admissible Drinfeld-Yetter modules over $B$ is equivalent, as a braided tensor category, to that of modules over the quantum double $D B$ of $B$ (cf. $\S$ A.5 for a detailed proof).

2.21. Admissibility criterion. By definition of $B^{\prime}$, and the exactness of the tensor product of topological vector spaces, a Drinfeld-Yetter module $\mathcal{V}$ is admissible if, and only if, for any $n \geq 1$, the image of

$$
(\mathrm{id}-\iota \circ \epsilon)^{\otimes n} \circ \Delta^{(n)} \otimes \mathrm{id}_{\mathcal{V}} \circ \pi_{\mathcal{V}}^{*}
$$

lies in $\hbar^{n} B^{\otimes n} \otimes \mathcal{V}$. The following gives a more economical criterion.

Proposition. A Drinfeld-Yetter module $\left(\mathcal{V}, \pi_{\mathcal{V}}, \pi_{\mathcal{V}}^{*}\right)$ is admissible if, and only if

$$
\operatorname{Im}\left((\mathrm{id}-\iota \circ \epsilon) \otimes \mathrm{id} \circ \pi_{\mathcal{V}}^{*}\right) \subset \hbar B \otimes \mathcal{V} .
$$

Proof. If $\mathcal{V}$ is admissible, its coaction factors through $B^{\prime} \otimes \mathcal{V}$ and (2.9) follows by the definition of $B^{\prime}$. Conversely, assume $\pi_{\mathcal{V}}^{*}$ satisfies (2.9). Then, it follows from (2.6) that $\Delta^{(n)} \otimes \operatorname{id}_{\mathcal{V}} \circ \pi_{\mathcal{V}}^{*}=\sigma \circ\left(\pi_{\mathcal{V}}^{*}\right)^{(n)}$ for some $\sigma \in \mathfrak{S}_{n}$. In particular, (2.9) implies that

$$
\operatorname{Im}\left((\mathrm{id}-\iota \circ \epsilon)^{\otimes n} \otimes \mathrm{id}_{\mathcal{V}} \circ \Delta^{(n)} \otimes \mathrm{id} \circ \pi_{\mathcal{V}}^{*}\right) \subset \hbar^{n} B^{\otimes n} \otimes \mathcal{V}
$$

2.22. Semiclassical limit of admissible Drinfeld-Yetter modules. Let $B$ be a QUE, with underlying Lie bialgebra $(\mathfrak{b},[],, \delta)$. The category of admissible Drinfeld-Yetter modules over $B$ is a quantisation of the category of Drinfeld-Yetter modules over $\mathfrak{b}$. Specifically, for any $\mathcal{V} \in D Y_{B}^{\text {adm }}$ with action and coaction

$$
\pi_{\mathcal{V}}: B \otimes \mathcal{V} \rightarrow \mathcal{V} \quad \text { and } \quad \pi_{\mathcal{V}}^{*}: \mathcal{V} \rightarrow B^{\prime} \otimes \mathcal{V}
$$

we set $\mathrm{SC}(\mathcal{V})=\mathcal{V} / \hbar \mathcal{V}$ and define the maps

$$
\mathrm{SC}\left(\pi_{\mathcal{V}}\right): \mathfrak{b} \otimes \mathrm{SC}(\mathcal{V}) \rightarrow \mathrm{SC}(\mathcal{V}) \quad \text { and } \quad \mathrm{SC}\left(\pi_{\mathcal{V}}^{*}\right): \operatorname{SC}(\mathcal{V}) \rightarrow \mathfrak{b} \otimes \operatorname{SC}(\mathcal{V})
$$

as follows. For every $b \in \mathfrak{b}$ and $v \in \mathrm{SC}(\mathcal{V})$ we set

$$
\operatorname{SC}\left(\pi_{\mathcal{V}}\right) b \otimes v=\pi_{\mathcal{V}} \widetilde{b} \otimes \widetilde{v} \bmod \hbar
$$

where $\widetilde{b} \in B$ and $\widetilde{v} \in \mathcal{V}$ are arbitrarily lifts of $b, v$, and

$$
\mathrm{SC}\left(\pi_{\mathcal{V}}^{*}\right) v=\frac{1}{\hbar}\left(\operatorname{id}_{B}-\iota_{B} \circ \epsilon_{B}\right) \otimes \operatorname{id} \circ \pi_{\mathcal{V}}^{*} \widetilde{v} \bmod \hbar .
$$

Note that the map $\mathrm{SC}\left(\pi_{\mathcal{V}}^{*}\right)$ is well-defined since $\pi_{\mathcal{V}}^{*}$ is an admissible coaction. Namely, set $p_{B}^{(n)}=(\mathrm{id}-\iota \circ \epsilon)^{\otimes n} \Delta^{(n)}$. By definition, $x \in B^{\prime}$ if and only if $p_{B}^{(n)}(x) \in$ $\hbar^{n} B^{\otimes n}$ for any $n \geq 0$. It is easy to verify that

$$
\Delta\left(p_{B}(x)\right)=p_{B}^{(2)}(x)+1 \otimes p_{B}(x)+p_{B}(x) \otimes 1 .
$$

It follows that $\hbar^{-1} p_{B}(x)$ modulo $\hbar$ is primitive, and therefore belongs to $\mathfrak{b}$.

Proposition. $\left(\mathrm{SC}(\mathcal{V}), \mathrm{SC}\left(\pi_{\mathcal{V}}\right), \mathrm{SC}\left(\pi_{\mathcal{V}}^{*}\right)\right)$ is a Drinfeld-Yetter module over $\mathfrak{b}$. 
Proof. Set $V=\operatorname{SC}(\mathcal{V}), \pi_{V}=\operatorname{SC}\left(\pi_{\mathcal{V}}\right)$, and $\pi_{V}^{*}=\mathrm{SC}\left(\pi_{\mathcal{V}}^{*}\right)$. It is clear that $\left(V, \pi_{V}\right)$ is a $\mathfrak{b}$-module. To prove that $\left(V, \pi_{V}^{*}\right)$ is a $\mathfrak{b}$-comodule it is enough to observe that

$$
\left.\tilde{p} \otimes \tilde{p} \circ\left(\Delta_{B}-\Delta_{B}^{21}\right)\right|_{B^{\prime}}=\left.\delta \circ \widetilde{p}\right|_{B^{\prime}} \bmod \hbar
$$

where $\widetilde{p}=\hbar^{-1} p_{B}$. Finally, we have to prove the compatibility between $\pi_{V}$ and $\pi_{V}^{*}$, i.e.,

$$
\pi_{V}^{*} \circ \pi_{V}=\mathrm{id} \otimes \pi_{V} \circ(12) \circ \mathrm{id} \otimes \pi_{V}^{*}+[,] \otimes \mathrm{id} \circ \mathrm{id} \otimes \pi_{V}^{*}-\mathrm{id} \otimes \pi_{V} \circ \delta \otimes \mathrm{id} .
$$

This follows by studying modulo $\hbar^{2}$ the relation (2.7) on the vector $\widetilde{b} \otimes \widetilde{v}$. More specifically, one has

$$
S^{-1} \otimes(23) \circ \Delta^{(3)}(\widetilde{b})=S^{-1} \otimes \mathrm{id}^{\otimes 2} \circ \Delta^{(3)}(\widetilde{b})-\hbar 1 \otimes \delta(b) \bmod \hbar^{2} .
$$

Therefore, (2.7) reads

$$
\begin{aligned}
\pi_{\mathcal{V}}^{*} \circ \pi_{\mathcal{V}}(\widetilde{b} \otimes \widetilde{v})=m^{(3)} \otimes \pi_{\mathcal{V}} \circ(1342) \circ S^{-1} \otimes \mathrm{id}^{\otimes 4} \circ \Delta^{(3)} \otimes \pi_{\mathcal{V}}^{*}(\widetilde{b} \otimes \widetilde{v}) \\
-\hbar m^{(3)} \otimes \pi_{\mathcal{V}} \circ(1342) \circ 1 \otimes \delta \otimes \pi_{\mathcal{V}}^{*}(b \otimes \widetilde{v}) \quad \bmod \hbar^{2} .
\end{aligned}
$$

Now, since we are working modulo $\hbar^{2}$, it is enough to consider the coaction up to its linear term

$$
\pi_{\mathcal{V}}^{*}(\widetilde{v})=1 \otimes \widetilde{v}+\hbar \widetilde{v}_{1} \otimes \widetilde{v}_{0} \bmod \hbar^{2} .
$$

Then, we see that

$$
m^{(3)} \otimes \pi_{\mathcal{V}} \circ(1342) \circ S^{-1} \otimes \mathrm{id}^{\otimes 4} \circ \Delta^{(3)} \otimes \mathrm{id}^{\otimes 2}(\widetilde{b} \otimes 1 \otimes \widetilde{v})=\epsilon\left(\widetilde{b}_{1}\right) 1 \otimes \pi_{\mathcal{V}}\left(\widetilde{b_{2}} \otimes \widetilde{v}\right),
$$

which vanishes after composition with $\widetilde{p} \otimes \mathrm{id}$, and

$$
\begin{aligned}
m^{(3)} & \otimes \pi_{\mathcal{V}} \circ(1342) \circ S^{-1} \otimes \mathrm{id}^{\otimes 4} \circ \Delta^{(3)} \otimes \mathrm{id}^{\otimes 2}\left(\hbar \widetilde{b} \otimes \widetilde{v}_{1} \otimes \widetilde{v}_{0}\right) \\
& =m_{0}^{(3)} \otimes \pi_{\mathcal{V}} \circ(1342) \circ S_{0} \otimes \mathrm{id}^{\otimes 4} \circ \Delta_{0}^{(3)} \otimes \mathrm{id}^{\otimes 2}\left(\hbar \widetilde{b} \otimes \widetilde{v}_{1} \otimes \widetilde{v}_{0}\right) \quad \bmod \hbar^{2},
\end{aligned}
$$

which reduces to

$$
\left.\hbar \widetilde{v}_{1} \otimes \pi_{\mathcal{V}}\left(b \otimes \widetilde{v}_{0}\right)+\hbar(b \widetilde{v})_{1}-\widetilde{v}_{1} b\right) \otimes \widetilde{v}_{0} .
$$

After composition with $\widetilde{p} \otimes$ id and modulo $\hbar$, this gives

$$
\text { id } \otimes \pi_{V} \circ(12) \circ \text { id } \otimes \pi_{V}^{*}+[,] \otimes \text { id } \circ \text { id } \otimes \pi_{V}^{*}
$$

Finally, one has

$$
-\hbar m^{(3)} \otimes \pi_{\mathcal{V}} \circ(1342) \circ 1 \otimes \delta \otimes \mathrm{id}^{\otimes 2}(b \otimes 1 \otimes \widetilde{v})=-\hbar \mathrm{id} \otimes \pi_{\mathcal{V}} \circ \delta(b \otimes \widetilde{v})
$$

which, after composition with $\widetilde{p} \otimes$ id and modulo $\hbar$, gives

$$
-\mathrm{id} \otimes \pi_{V} \circ \delta(b \otimes v)
$$

and proves the compatibility (2.10). 
2.23. Quantisation of representations. We now return to the setting of 2.16. Let $U, V \in \mathrm{DY}_{\mathfrak{b}}^{\Phi}=\mathcal{E}_{\mathfrak{g}_{\mathfrak{b}}}^{\Phi}$, and $R_{U, V}^{J} \in \operatorname{End}_{\mathfrak{K}}\left(F_{\mathfrak{b}}(U) \otimes F_{\mathfrak{b}}(V)\right)$ the twisted $R$-matrix defined by the diagram

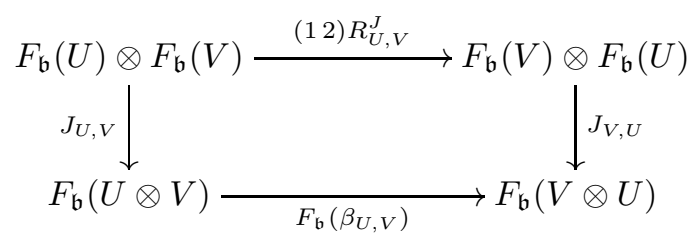

where $\beta_{U, V}=(12) \exp ^{\hbar / 2} \Omega_{U, V}$ is the commutativity constraint in $\mathrm{DY}_{\mathfrak{b}}^{\Phi}$. Then, the map

$$
m_{V}^{*}: F_{\mathfrak{b}}(V) \rightarrow F_{\mathfrak{b}}\left(M_{-}\right) \otimes F_{\mathfrak{b}}(V) \quad m_{V}^{*}(v)=R_{M_{-}, V}^{J} u_{-} \otimes v
$$

defines a right coaction of $F_{\mathfrak{b}}\left(M_{-}\right)$on $F_{\mathfrak{b}}(V)$, which is compatible with the left action of $F_{\mathfrak{b}}\left(M_{-}\right)$on $V$ given by $m_{V}$ in the sense of $(2.7) .\left(F_{\mathfrak{b}}(V), m_{V}, m_{V}^{*}\right)$ is therefore a Drinfeld-Yetter module over $F_{\mathfrak{b}}\left(M_{-}\right)$.

\section{Lemma.}

(i) The Drinfeld-Yetter module $\widetilde{F}_{\mathfrak{b}}(V)=\left(F_{\mathfrak{b}}(V), m_{V}, m_{V}^{*}\right)$ is admissible.

(ii) The semiclassical limit of $\widetilde{F}_{\mathfrak{b}}(V)$ is equal to $V \in \mathrm{DY}_{\mathfrak{b}}$.

Proof. (i) We have to show that the coaction defined through the twisted $R$ matrix is admissible, i.e., it factors through $\left(U_{\hbar} \mathfrak{b}\right)^{\prime} \otimes F_{b}(V)$. It follows from [10] and [3, Section 3.2] that the action of $R^{J}$ can be written as an infinite sum of elements of the form

$$
\hbar^{N} x_{1} \ldots x_{k} \cdot y_{1} \cdots y_{s} \otimes x_{k+1} \cdots x_{N} \cdot y_{s+1} \cdots y_{N}
$$

with $x_{i} \in \mathfrak{b}, y_{j} \in \mathfrak{b}^{*}$. Since $\mathfrak{b}^{*}$ acts trivially on $u_{-}$, it follows that the first component of $R_{M_{-}, V}^{J} u_{-} \otimes v$ lies in $\left(U_{\hbar} \mathfrak{b}\right)^{\prime}$.

(ii) It is enough to observe from the formulae in $\S 2.16$ and $\S 2.22$ that the quantum action $m_{V}$ reduces to $\pi_{V}$ modulo $\hbar$. Similarly the quantum coaction $m_{V}^{*}$ defined by the $R$-matrix satisfies

$$
\frac{1}{\hbar}(\mathrm{id}-\varepsilon) \otimes \mathrm{id} \circ R_{M_{-}, V}^{J} \circ u_{-} \otimes \mathrm{id}=r_{M_{-}, V} \circ u_{-} \otimes \mathrm{id}=\pi_{V}^{*} \quad \bmod \hbar
$$

and gives back the coaction $\pi_{V}^{*}$ on $V$.

\subsection{Tannakian equivalence.}

Theorem. [16] The fiber functor $F_{\mathfrak{b}}: \mathrm{DY}_{\mathfrak{b}}^{\Phi} \rightarrow$ Vect $_{\mathfrak{K}}$ lifts to an equivalence of braided tensor categories $\widetilde{F}_{\mathfrak{b}}: \mathrm{DY}_{\mathfrak{b}}^{\Phi} \rightarrow \mathrm{DY}_{U_{\hbar} \mathfrak{b}}^{\mathrm{adm}}$.

The proof of Theorem 2.24 is given in $[15,16]$, and relies on the action of the Grothendieck-Teichmuller group on the braided tensor structure of DY $Y_{\mathfrak{b}}^{\Phi}$ and $D Y_{U_{\hbar} \mathfrak{b}}^{\text {adm }}{ }^{7}$ We sketch an alternative proof in $\S 6.17$, which relies on a PROPic interpretation of the functor $\widetilde{F}_{\mathfrak{b}}$.

\footnotetext{
${ }^{7}$ We are grateful to Pavel Etingof for pointing out that, contrary to what is stated in [16, §4.1], the functor $\widetilde{F}_{\mathfrak{b}}$ is not an equivalence if the target category is taken to be Drinfeld-Yetter modules over $U_{\hbar} \mathfrak{b}$, and that attention needs to be restricted to admissible ones.
} 
2.25. Quantisation of bimodules. For later use, we will need to consider the quantisation of certain Drinfeld-Yetter bimodules. Given two Lie bialgebras $\mathfrak{c}$ and $\mathfrak{d}$ (resp. Hopf algebras $C$ and $D$ ), a Drinfeld-Yetter $(\mathfrak{c}, \mathfrak{d})$-module (resp. $(C, D)-$ module) is a vector space endowed with commuting actions and coactions over $\mathfrak{c}$ and $\mathfrak{d}$ (resp. $C$ and $D$ ). It is immediate to verify that there are canonical equivalences

$$
\begin{aligned}
\mathrm{DY}_{(\mathfrak{c}, \mathfrak{d})} & \simeq \mathrm{DY}_{\mathfrak{c} \oplus \mathfrak{d}^{\mathrm{op}}} \\
\mathrm{DY}_{(C, D)} & \simeq \mathrm{DY}_{C \otimes D^{\mathrm{op}}}
\end{aligned}
$$

where $\mathrm{DY}_{(\mathfrak{c}, \mathfrak{d})}$ and $\mathrm{DY}_{(C, D)}$ denote the categories of bimodules, $\mathfrak{c} \oplus \mathfrak{d}^{\mathrm{op}}$ is the direct sum Lie bialgebra, and $C \otimes D^{\mathrm{op}}$ is the tensor Hopf algebra.

Proposition. There is a commutative diagram of braided tensor functors

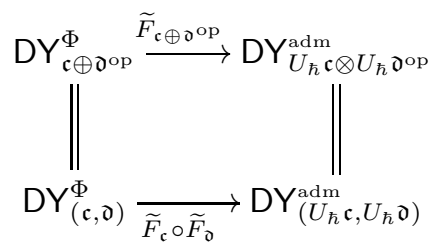

and similarly with $\widetilde{F}_{\mathfrak{c}} \circ \widetilde{F}_{\mathfrak{d}}$ replaced by $\widetilde{F}_{\mathfrak{d}} \circ \widetilde{F}_{\mathfrak{c}}$.

Proof. Let $M_{\mathfrak{c} \oplus \mathfrak{d}^{\text {op }}}, M_{\mathfrak{c}}, M_{\mathfrak{d}^{\text {op }}}$ (resp. $\quad M_{\mathfrak{c} \oplus \mathfrak{d}^{\text {op }}}^{\vee}, M_{\mathfrak{c}}^{\vee}, M_{\mathfrak{d}^{\text {op }}}^{\vee}$ ) be the Verma modules $M_{-}$(resp. $M_{+}^{\vee}$ ) corresponding to the Lie bialgebras $\mathfrak{c} \oplus \mathfrak{d}^{\mathrm{op}}, \mathfrak{c}$, and $\mathfrak{d}^{\mathrm{op}}$. As $\mathfrak{c} \oplus \mathfrak{d}^{\mathrm{op}}$ modules, $M_{\mathfrak{c} \oplus \mathfrak{d}^{\text {op }}} \simeq M_{\mathfrak{c}} \otimes M_{\mathfrak{o}^{\text {op }}}$ and $M_{\mathfrak{c} \oplus \mathfrak{d}^{\text {op }}}^{\vee} \simeq M_{\mathfrak{c}}^{\vee} \otimes M_{\mathfrak{d}^{\text {op }}}^{\vee}$. Moreover, there is an isomorphism of functors

$$
\begin{aligned}
& \widetilde{F}_{\mathfrak{c} \oplus \mathfrak{d}^{\mathrm{op}}}(V)=\operatorname{Hom}_{\mathfrak{c} \oplus \mathfrak{d}^{\mathrm{op}}}^{\mathfrak{c} \oplus{ }^{\mathrm{op}}}\left(M_{\mathfrak{c}} \otimes M_{\mathfrak{d} \text { op }}, M_{\mathfrak{c}}^{\vee} \otimes M_{\mathfrak{d}^{\mathrm{op}}}^{\vee} \otimes V\right) \\
& \simeq \operatorname{Hom}_{\mathfrak{c}}^{\mathfrak{c}}\left(M_{\mathfrak{c}}, \operatorname{Hom}_{\mathfrak{d}^{\mathrm{op}}}^{\mathfrak{o}^{\mathrm{op}}}\left(M_{\mathfrak{d}^{\mathrm{op}}}, M_{\mathfrak{c}}^{\vee} \otimes M_{\mathfrak{d}^{\mathrm{op}}}^{\vee} \otimes V\right)\right) \\
& \simeq \operatorname{Hom}_{\mathfrak{c}}^{\mathfrak{c}}\left(M_{\mathfrak{c}}, M_{\mathfrak{c}}^{\vee} \otimes \operatorname{Hom}_{\mathfrak{d}^{\text {op }}}^{\mathfrak{o}^{\text {op }}}\left(M_{\mathfrak{d}^{\text {op }}}, M_{\mathfrak{d}^{\text {op }}}^{\vee} \otimes V\right)\right)=\widetilde{F}_{\mathfrak{c}} \circ \widetilde{F}_{\mathfrak{d}^{\text {op }}}(V)
\end{aligned}
$$

where the first identification follows by adjointness and the second one follows by the isomorphism of Drinfeld-Yetter $\mathfrak{c}$-modules between $\operatorname{Hom}_{\mathfrak{d}^{\mathrm{op}}}^{\mathfrak{o}^{\mathrm{op}}}\left(M_{\mathfrak{d}^{\mathrm{op}}}, M_{\mathfrak{c}}^{\vee} \otimes M_{\mathfrak{d}^{\circ \mathrm{p}}}^{\vee} \otimes\right.$ $V)$ and $M_{\mathfrak{c}}^{\vee} \otimes \operatorname{Hom}_{\mathfrak{d}^{\mathrm{op}}}^{\mathfrak{o}^{\mathrm{op}}}\left(M_{\mathfrak{D}^{\mathrm{op}}}, M_{\mathfrak{D}^{\mathrm{op}}}^{\vee} \otimes V\right)$. It is straightforward to verify that the isomorphism $\widetilde{F}_{\mathfrak{c} \oplus \mathfrak{d}^{\circ \mathrm{p}}} \simeq \widetilde{F}_{\mathfrak{c}} \circ \widetilde{F}_{\mathfrak{d}^{\text {op }}}$ preserves the tensor structure.

\section{Tensor Structures on Restriction FunCtors}

3.1. In this section, we consider a split inclusion of Manin triples

$$
i:\left(\mathfrak{g}_{\mathfrak{a}}, \mathfrak{a}_{-}, \mathfrak{a}_{+}\right) \hookrightarrow\left(\mathfrak{g}_{\mathfrak{b}}, \mathfrak{b}_{-}, \mathfrak{b}_{+}\right) .
$$

We then define a relative version of the Verma modules $M_{ \pm}$, and use them to prove the following

Theorem. There is a tensor functor

$$
\left(F_{\mathfrak{a}, \mathfrak{b}}, J_{\mathfrak{a}, \mathfrak{b}}\right): \mathcal{E}_{\mathfrak{g}_{\mathfrak{b}}}^{\Phi} \longrightarrow \mathcal{E}_{\mathfrak{g}_{\mathfrak{a}}}^{\Phi}
$$

such that $F_{\mathfrak{a}, \mathfrak{b}}$ is isomorphic to the restriction functor $i^{*}$. 


\subsection{Split inclusions of Manin triples.}

Definition. An embedding of Manin triples

$$
i:\left(\mathfrak{g}_{\mathfrak{a}}, \mathfrak{a}_{-}, \mathfrak{a}_{+}\right) \longrightarrow\left(\mathfrak{g}_{\mathfrak{b}}, \mathfrak{b}_{-}, \mathfrak{b}_{+}\right)
$$

is a continuous Lie algebra homomorphism $i: \mathfrak{g}_{\mathfrak{a}} \rightarrow \mathfrak{g}_{\mathfrak{b}}$ preserving inner products, and such that $i\left(\mathfrak{a}_{ \pm}\right) \subset \mathfrak{b}_{ \pm}$.

Denote the restriction of $i$ to $\mathfrak{a}_{ \pm}$by $i_{ \pm}: \mathfrak{a}_{ \pm} \rightarrow \mathfrak{b}_{ \pm} . i_{ \pm}$are continuous embeddings, and give rise to maps $p_{ \pm}=i_{\mp}^{t}: \mathfrak{b}_{ \pm} \rightarrow \mathfrak{a}_{ \pm}$, defined via the identifications $\mathfrak{b}_{ \pm} \simeq \mathfrak{b}_{\mp}^{*}$ and $\mathfrak{a}_{ \pm} \simeq \mathfrak{a}_{\mp}^{*}$ by $\left\langle p_{ \pm}(x), y\right\rangle=\left\langle x, i_{\mp}(y)\right\rangle$ for any $x \in \mathfrak{g}_{ \pm}$and $y \in \mathfrak{a}_{\mp}$. These maps satisfy $p_{ \pm} \circ i_{ \pm}=\operatorname{id}_{\mathfrak{a}_{ \pm}}$since, for any $x \in \mathfrak{a}_{ \pm}, y \in \mathfrak{a}_{\mp}$,

$$
\left\langle p_{ \pm} \circ i_{ \pm}(x), y\right\rangle=\left\langle i_{ \pm}(x), i_{\mp}(y)\right\rangle .
$$

This yields in particular a direct sum decomposition $\mathfrak{b}_{ \pm}=i\left(\mathfrak{a}_{ \pm}\right) \oplus \mathfrak{m}_{ \pm}$, where

$$
\mathfrak{m}_{ \pm}=\operatorname{Ker}\left(p_{ \pm}\right)=\mathfrak{b}_{ \pm} \cap i\left(\mathfrak{g}_{\mathfrak{a}}\right)^{\perp}
$$

Definition. The embedding $i:\left(\mathfrak{g}_{\mathfrak{a}}, \mathfrak{a}_{-}, \mathfrak{a}_{+}\right) \longrightarrow\left(\mathfrak{g}_{\mathfrak{b}}, \mathfrak{b}_{-}, \mathfrak{b}_{+}\right)$is called split if the subspaces $\mathfrak{m}_{ \pm} \subset \mathfrak{b}_{ \pm}$are Lie subalgebras.

3.3. Split pairs of Lie bialgebras. We now reformulate the above notion in terms of bialgebras.

Definition. A split pair of Lie bialgebras is the data of

- Lie bialgebras $\mathfrak{a}, \mathfrak{b}$.

- Lie bialgebra morphisms $i: \mathfrak{a} \rightarrow \mathfrak{b}$ and $p: \mathfrak{b} \rightarrow \mathfrak{a}$ such that $p \circ i=\operatorname{id}_{\mathfrak{a}}$.

Proposition. There is a one-to-one correspondence between split inclusions of Manin triples and split pairs of Lie bialgebras. Specifically,

(i) If $i:\left(\mathfrak{g}_{\mathfrak{a}}, \mathfrak{a}_{-}, \mathfrak{a}_{+}\right) \longrightarrow\left(\mathfrak{g}_{\mathfrak{b}}, \mathfrak{b}_{-}, \mathfrak{b}_{+}\right)$is a split inclusion of Manin triples, then $\left(\mathfrak{a}_{-}, \mathfrak{b}_{-}, i_{-}, i_{+}^{*}\right)$ is a split pair of Lie bialgebras.

(ii) Conversely, if $(\mathfrak{a}, \mathfrak{b}, i, p)$ is a split pair of Lie bialgebras, then $i \oplus p^{*}$ : $\left(\mathfrak{g}_{\mathfrak{a}}, \mathfrak{a}, \mathfrak{a}^{*}\right) \longrightarrow\left(\mathfrak{g}_{\mathfrak{b}}, \mathfrak{b}, \mathfrak{b}^{*}\right)$ is a split inclusion of Manin triples.

3.4. Proof of (i) of Proposition 3.3. Given a split inclusion

$$
i=i_{-} \oplus i_{+}:\left(\mathfrak{g}_{\mathfrak{a}}, \mathfrak{a}_{-}, \mathfrak{a}_{+}\right) \longrightarrow\left(\mathfrak{g}_{\mathfrak{b}}, \mathfrak{b}_{-}, \mathfrak{b}_{+}\right)
$$

we need to show that $i_{-}$and $i_{+}^{*}$ are Lie bialgebra morphisms. By assumption, $i_{-}$is a morphism of Lie algebras, and $i_{+}^{*}$ one of coalgebras. Since $i_{-}=\left(i_{-}^{*}\right)^{*}$, it suffices to show that $p_{ \pm}=i_{\mp}^{*}$ preserve Lie brackets.

We claim to this end that $\mathfrak{m}_{ \pm}$are ideals in $\mathfrak{b}_{ \pm}$. Since $\left[\mathfrak{m}_{ \pm}, \mathfrak{m}_{ \pm}\right] \subseteq \mathfrak{m}_{ \pm}$by assumption, this amounts to showing that $\left[i\left(\mathfrak{a}_{ \pm}\right), \mathfrak{m}_{ \pm}\right] \subseteq \mathfrak{m}_{ \pm}$. This follows from the fact that $\left[i\left(\mathfrak{a}_{ \pm}\right), \mathfrak{m}_{ \pm}\right] \subseteq \mathfrak{b}_{ \pm}$, and from

$$
\left\langle\left[i\left(\mathfrak{a}_{ \pm}\right), \mathfrak{m}_{ \pm}\right], i\left(\mathfrak{a}_{\mp}\right)\right\rangle=\left\langle\mathfrak{m}_{ \pm},\left[i\left(\mathfrak{a}_{ \pm}\right), i\left(\mathfrak{a}_{\mp}\right)\right]\right\rangle \subset\left\langle\mathfrak{m}_{ \pm}, i\left(\mathfrak{a}_{ \pm}\right)\right\rangle+\left\langle\mathfrak{m}_{ \pm}, i\left(\mathfrak{a}_{\mp}\right)\right\rangle,
$$

where the first term is zero since $\mathfrak{b}_{ \pm}$is isotropic, and the second one is zero by definition of $\mathfrak{m}_{ \pm}$.

Let now $X_{1}, X_{2} \in \mathfrak{b}_{ \pm}$, and write $X_{j}=i_{ \pm}\left(x_{j}\right)+y_{j}$, where $x_{j} \in \mathfrak{a}_{ \pm}$and $y_{j} \in \mathfrak{m}_{ \pm}$. Since $\mathfrak{m}_{ \pm}=\operatorname{Ker}\left(p_{ \pm}\right)$and $p_{ \pm} \circ i_{ \pm}=\mathrm{id}$, we have $\left[p_{ \pm}\left(X_{1}\right), p_{ \pm}\left(X_{2}\right)\right]=\left[x_{1}, x_{2}\right]$, while

$$
p_{ \pm}\left[X_{1}, X_{2}\right]=p_{ \pm}\left(i_{ \pm}\left[x_{1}, x_{2}\right]+\left[i_{ \pm} x_{1}, y_{2}\right]+\left[y_{1}, i_{ \pm} x_{2}\right]+\left[y_{1}, y_{2}\right]\right)=\left[x_{1}, x_{2}\right],
$$

where the last equality follows from the fact that $\mathfrak{m}_{ \pm}$is an ideal. 
3.5. Proof of (ii) of Proposition 3.3. The bracket on $\mathfrak{g}_{\mathfrak{a}}$ is defined by

$$
[a, \phi]=\operatorname{ad}^{*}(a)(\phi)-\operatorname{ad}^{*}(\phi)(a)=-\left\langle\phi,[a,-]_{\mathfrak{a}}\right\rangle+\left\langle\phi \otimes \mathrm{id}, \delta_{\mathfrak{a}}(a)\right\rangle
$$

for any $a \in \mathfrak{a}, \phi \in \mathfrak{a}^{*}$. Analogously for $\mathfrak{g}_{\mathfrak{b}}$. Therefore, the equalities

$$
\begin{aligned}
\left\langle p^{*}(\phi) \otimes \mathrm{id}, \delta_{\mathfrak{b}}(i(a))\right\rangle & =\left\langle\phi \otimes \mathrm{id},(p \otimes \mathrm{id})(i \otimes i) \delta_{\mathfrak{a}}(a)\right\rangle \\
& =\left\langle\phi \otimes \mathrm{id},(\mathrm{id} \otimes i) \delta_{\mathfrak{a}}(a)\right\rangle=i\left(\left\langle\phi \otimes \mathrm{id}, \delta_{\mathfrak{a}}(a)\right\rangle\right)
\end{aligned}
$$

and

$$
\left\langle p^{*}(\phi),[i(a), b]_{\mathfrak{b}}\right\rangle=\left\langle\phi, p\left([i(a), b]_{\mathfrak{b}}\right)\right\rangle=\left\langle\phi,[a, p(b)]_{\mathfrak{a}}\right\rangle
$$

for all $a \in \mathfrak{a}$ and $b \in \mathfrak{b}$, imply that the map $i \oplus p^{*}$ is a Lie algebra map. It also respects the inner product, since for any $a \in \mathfrak{a}, \phi \in \mathfrak{a}^{*}$,

$$
\left\langle p^{*}(\phi), i(a)\right\rangle=\langle\phi, p \circ i(a)\rangle=\langle\phi, a\rangle
$$

Finally, $\mathfrak{m}_{-}=\operatorname{Ker}(p)$ and $\mathfrak{m}_{+}=\operatorname{Ker}\left(i^{*}\right)$ are clearly subalgebras.

\subsection{Parabolic Lie subalgebras. Let}

$$
i=i_{-} \oplus i_{+}:\left(\mathfrak{g}_{\mathfrak{a}}, \mathfrak{a}_{-}, \mathfrak{a}_{+}\right) \rightarrow\left(\mathfrak{g}_{\mathfrak{b}}, \mathfrak{b}_{-}, \mathfrak{b}_{+}\right)
$$

be a split embedding of Manin triples. The following summarizes the properties of the subspaces

$$
\mathfrak{m}_{ \pm}=\mathfrak{b}_{ \pm} \cap i\left(\mathfrak{g}_{\mathfrak{a}}\right)^{\perp} \quad \text { and } \quad \mathfrak{p}_{ \pm}=\mathfrak{m}_{ \pm} \oplus i\left(\mathfrak{g}_{\mathfrak{a}}\right)
$$

\section{Proposition.}

(i) $\mathfrak{m}_{ \pm}$is an ideal in $\mathfrak{b}_{ \pm}$, so that $\mathfrak{b}_{ \pm}=\mathfrak{m}_{ \pm} \rtimes i_{ \pm}\left(\mathfrak{a}_{ \pm}\right)$.

(ii) $\left[i\left(\mathfrak{g}_{\mathfrak{a}}\right), \mathfrak{m}_{ \pm}\right] \subset \mathfrak{m}_{ \pm}$, so that $\mathfrak{p}_{ \pm}=\mathfrak{m}_{ \pm} \rtimes i\left(\mathfrak{g}_{\mathfrak{a}}\right)$ are Lie subalgebras of $\mathfrak{g}_{\mathfrak{b}}$.

(iii) $\delta\left(\mathfrak{m}_{-}\right) \subset \mathfrak{m}_{-} \otimes i_{-}\left(\mathfrak{a}_{-}\right)+i_{-}\left(\mathfrak{a}_{-}\right) \otimes \mathfrak{m}_{-}$, so that $\mathfrak{m}_{-} \subseteq \mathfrak{b}_{-}$is a coideal.

Proof. (i) was proved in $§ 3.4$. (ii) Since

$$
\left\langle\left[i\left(\mathfrak{g}_{\mathfrak{a}}\right), \mathfrak{m}_{ \pm}\right], i\left(\mathfrak{g}_{\mathfrak{a}}\right)\right\rangle=\left\langle\mathfrak{m}_{ \pm},\left[i\left(\mathfrak{g}_{\mathfrak{a}}\right), i\left(\mathfrak{g}_{\mathfrak{a}}\right)\right]\right\rangle=0,
$$

we have $\left[i\left(\mathfrak{g}_{\mathfrak{a}}\right), \mathfrak{m}_{ \pm}\right] \subset i\left(\mathfrak{g}_{\mathfrak{a}}\right)^{\perp}=\mathfrak{m}_{-} \oplus \mathfrak{m}_{+}$. Moreover,

$$
\left\langle\left[i\left(\mathfrak{g}_{\mathfrak{a}}\right), \mathfrak{m}_{ \pm}\right], \mathfrak{m}_{ \pm}\right\rangle=\left\langle i\left(\mathfrak{g}_{\mathfrak{a}}\right),\left[\mathfrak{m}_{ \pm}, \mathfrak{m}_{ \pm}\right]\right\rangle=\left\langle i\left(\mathfrak{g}_{\mathfrak{a}}\right), \mathfrak{m}_{ \pm}\right\rangle=0,
$$

since $\mathfrak{m}_{ \pm}$is a subalgebra, and it follows that $\left[i\left(\mathfrak{g}_{\mathfrak{a}}\right), \mathfrak{m}_{ \pm}\right] \subset \mathfrak{m}_{ \pm}$. (iii) is clear since $\mathfrak{m}_{-}$is the kernel of a Lie coalgebra map.

\subsection{The relative Verma Modules.}

Definition. Given a split embedding of Manin triples $i: \mathfrak{g}_{\mathfrak{a}} \rightarrow \mathfrak{g}_{\mathfrak{b}}$, and the corresponding decomposition $\mathfrak{g}_{\mathfrak{b}}=\mathfrak{m}_{-} \oplus \mathfrak{p}_{+}$, the relative Verma modules $L_{-}, N_{+}$are defined by

$$
L_{-}=\operatorname{Ind}_{\mathfrak{p}_{+}}^{\mathfrak{g}_{\mathfrak{b}}} \mathrm{k} \quad \text { and } \quad N_{+}=\operatorname{Ind}_{\mathfrak{m}_{-}}^{\mathfrak{g}_{\mathfrak{b}}} \mathrm{k} .
$$

Since $\mathfrak{p}_{+}$and $\mathfrak{m}_{-}$are invariant under the adjoint action of $i\left(\mathfrak{g}_{\mathfrak{a}}\right)$, the right action of $\mathfrak{g}_{\mathfrak{a}}$ on $U \mathfrak{g}_{\mathfrak{b}}$ descends to one on $L_{-}$and $N_{+}$, so both are $\left(\mathfrak{g}_{\mathfrak{b}}, \mathfrak{g}_{\mathfrak{a}}\right)$-bimodules, with the right action of $\mathfrak{g}_{\mathfrak{a}}$ on $L_{-}$being trivial.

Proposition. The $\left(\mathfrak{g}_{\mathfrak{b}}, \mathfrak{g}_{\mathfrak{a}}\right)$-modules $L_{-}$and $N_{+}^{\vee}$ are equicontinuous.

The description of the appropriate topologies on $L_{-}$and $N_{+}^{\vee}$, and the proof of their equicontinuity will be carried out in $§ 3.8-\$ 3.11$. 
3.8. Equicontinuity of $L_{-}$. As vector spaces,

$$
L_{-} \simeq U \mathfrak{m}_{-} \subset U \mathfrak{b}_{-} .
$$

It is therefore natural to equip $L_{-}$with the discrete topology. The set of operators $\left\{\pi_{L_{-}}(x)\right\}_{x \in \mathfrak{g}_{\mathfrak{b}}}$ is then an equicontinuous family, and the continuity of $\pi_{L_{-}}$reduces to checking that, for every element $v \in L_{-}$, the set

$$
Z_{v}=\left\{X \in \mathfrak{g}_{\mathfrak{b}} \mid X v=0\right\}
$$

is a neighborhood of zero in $\mathfrak{g}_{\mathfrak{b}}$. Since $U \mathfrak{m}_{-}$embeds in $U \mathfrak{b}_{-}$, the proof is identical to that of [14, Lemma 7.2]. We proceed by induction on the length of $v=Y_{n} \cdots Y_{1} \mathbf{1}_{-}$, $Y_{i} \in \mathfrak{m}_{-}$. If $n=0$, then $v=\mathbf{1}_{-}$and $Z_{v}=\mathfrak{p}_{+}=i_{-}\left(\mathfrak{a}_{-}\right) \oplus \mathfrak{b}_{+}$is open in $\mathfrak{g}_{\mathfrak{b}}$. If $n \geq 1$, the identity

$$
X Y_{n} \cdots Y_{1} \mathbf{1}_{-}=Y_{n} X Y_{n-1} \cdots Y_{1} \mathbf{1}_{-}+\left[X, Y_{n}\right] Y_{n-1} \cdots Y_{1} \mathbf{1}_{-}
$$

shows that $Z_{Y_{n} \cdots Y_{1} \mathbf{1}_{-}} \supset Z_{Y_{n-1} \cdots Y_{1} \mathbf{1}_{-}} \cap \operatorname{ad}\left(Y_{n}\right)^{-1}\left(Z_{Y_{n-1} \cdots Y_{1} \mathbf{1}_{-}}\right)$, and the conclusion follows from the continuity of the bracket.

\subsection{Topology of $N_{+}$. As vector spaces,}

$$
N_{+}=\operatorname{Ind}_{\mathfrak{m}_{-}}^{\mathfrak{g}_{\mathfrak{b}}} \mathrm{k} \simeq U \mathfrak{p}_{+} \simeq \operatorname{colim} U_{n} \mathfrak{p}_{+},
$$

where $\left\{U_{n} \mathfrak{p}_{+}\right\}$denotes the standard filtration of $U \mathfrak{p}_{+}$, so that

$$
U_{n} \mathfrak{p}_{+} \simeq \bigoplus_{m=0}^{n} S^{m} \mathfrak{p}_{+}=\bigoplus_{i+j \leq n}\left(S^{i} \mathfrak{b}_{+} \otimes S^{j} \mathfrak{a}_{-}\right)
$$

We turn this isomorphism into an isomorphism of topological vector spaces, by taking on $S^{i} \mathfrak{b}_{+}$and $S^{j} \mathfrak{a}_{-}$the topologies induced by the embeddings

$$
S^{i} \mathfrak{b}_{+} \hookrightarrow\left(\mathfrak{b}_{-}^{\otimes i}\right)^{*} \quad \text { and } \quad S^{j} \mathfrak{a}_{-} \hookrightarrow\left(\mathfrak{a}_{-}^{\otimes j}\right)^{* *} .
$$

With respect to these topologies, $U_{m} \mathfrak{p}_{+}$is closed inside $U_{n} \mathfrak{p}_{+}$for $m<n$, and we equip $N_{+}$with the direct limit topology. We shall need the following

Lemma. For any $x \in \mathfrak{g}_{\mathfrak{b}}, y \in \mathfrak{g}_{\mathfrak{a}}$, the maps $\pi_{N_{+}}(x): N_{+} \rightarrow N_{+}$are continuous.

Proof. We need to show that for any neighborhood of the origin $U \subset N_{+}$, there exists a neighborhood of zero $U^{\prime} \subset N_{+}$such that $\pi_{N_{+}}(x) U^{\prime} \subset U$. The topology on $N_{+}$comes from the decomposition $U \mathfrak{p}_{+} \simeq U \mathfrak{b}_{+} \otimes U \mathfrak{a}_{-}$, so that an open neighborhood of zero in $N_{+}$has the form $U \otimes U \mathfrak{a}_{-}+U \mathfrak{b}_{+} \otimes V$, with $U$ open in $U \mathfrak{b}_{+}$and $V$ open in $U \mathfrak{a}_{-}$. We apply the same procedure used in [14, Lemma 7.3] to construct an open set $U^{\prime} \otimes U \mathfrak{a}_{-}$, with $U^{\prime}$ open in $U \mathfrak{b}_{+}$, such that

$$
\pi_{N_{+}}(x)\left(U^{\prime} \otimes U \mathfrak{a}_{-}\right) \subset U \otimes U \mathfrak{a}_{-} \subset U \otimes U \mathfrak{a}_{-}+U \mathfrak{b}_{+} \otimes V .
$$

3.10. Topology of $N_{+}^{\vee}$. As vector spaces, $N_{+}^{*} \simeq\left(U \mathfrak{p}_{+}\right)^{*} \simeq \lim \left(U_{n} \mathfrak{p}_{+}\right)^{*}$. In analogy with $\S 2.13$, we consider the discrete topology on $\left(U_{n} \mathfrak{p}_{+}\right)^{*}$ and the limit topology on $N_{+}^{*}$, denoting the resulting topological space by $N_{+}^{\vee}$. This defines a filtration of subspaces $\left\{\left(N_{+}^{\vee}\right)_{n}\right\}$ on $N_{+}^{\vee}$ by

$$
0 \rightarrow\left(N_{+}^{\vee}\right)_{n} \rightarrow\left(U \mathfrak{p}_{+}\right)^{*} \rightarrow\left(U_{n} \mathfrak{p}_{+}\right)^{*} \rightarrow 0,
$$

so that $N_{+}^{\vee} \supset\left(N_{+}^{\vee}\right)_{0} \supset\left(N_{+}^{\vee}\right)_{1} \supset \cdots$, and we get an isomorphism of vector spaces

$$
N_{+}^{\vee} \simeq \lim N_{+}^{\vee} /\left(N_{+}^{\vee}\right)_{n}
$$


Lemma. $\left\{\pi_{N_{+}^{\vee}}(x)\right\}_{x \in \mathfrak{g}}$ is an equicontinuous family of operators.

Proof. Since $\mathfrak{p}_{+}$acts on $N_{+}$by multiplication, $\mathfrak{p}_{+}\left(N_{+}^{\vee}\right)_{n} \subset\left(N_{+}^{\vee}\right)_{n-1}$. If $x \in \mathfrak{m}_{-}$ and $x_{i} \in U \mathfrak{p}_{+}$for $i=1, \ldots, n$, then in $U \mathfrak{g}_{\mathfrak{b}}$,

$$
x x_{1} \cdots x_{n}=x_{1} \cdots x_{n} x-\sum_{i=0}^{n} x_{1} \cdots x_{i-1}\left[x_{i}, x\right] x_{i+1} \cdots x_{n},
$$

where $\left[x_{i}, x\right] \in \mathfrak{g}_{\mathfrak{b}}$. Iterating shows that $(x . f)\left(x_{1} \cdots x_{n}\right)=0$ if $f \in\left(N_{+}^{\vee}\right)_{n}$, so that $x\left(N_{+}^{\vee}\right)_{n} \subset\left(N_{+}^{\vee}\right)_{n}$. Then, for any neighborhood of zero of the form $U=\left(N_{+}^{\vee}\right)_{n}$, it is enough to take $U^{\prime}=\left(N_{+}^{\vee}\right)_{n+1}$ to get $\mathfrak{g}_{\mathfrak{b}}\left(N_{+}^{\vee}\right)_{n+1} \subset\left(N_{+}^{\vee}\right)_{n}$.

3.11. Equicontinuity of $N_{+}^{\vee}$. In order to show that the module $N_{+}^{\vee}$ is equicontinuous it is enough to prove the following

Lemma. The map $\pi_{N_{+}^{\vee}}: \mathfrak{g}_{\mathfrak{b}} \rightarrow \operatorname{End}\left(N_{+}^{\vee}\right)$ is a continuous map.

Proof. Since $\mathfrak{b}_{-}$is discrete, it is enough to check that, for any $f \in N_{+}^{\vee}$ and $n \in \mathbb{N}$, the subset

$$
Y\left(F_{\mathfrak{b}}, n\right)=\left\{b \in \mathfrak{b}_{+} \mid b . f \in\left(N_{+}^{\vee}\right)_{n}\right\}
$$

is open in $\mathfrak{b}_{+}$, and $b^{i} . f \in\left(N_{+}^{\vee}\right)_{n}$ for all but finitely many $i \in I$. Since $f \in N_{+}^{\vee} \simeq$ $\lim N_{+}^{\vee} /\left(N_{+}^{\vee}\right)_{n}$, we have $f=\left\{f_{n}\right\}$ where $f_{n}$ is the class of $f$ modulo $\left(N_{+}^{\vee}\right)_{n}$. The classes $\left\{f_{n}\right\}$ are identified with elements in $\left(U_{n} \mathfrak{p}_{+}\right)^{*} \simeq \oplus\left(S^{j_{p_{+}}}\right)^{*} \simeq \oplus S^{j} \mathfrak{p}_{-}$and we denote by $T_{n}(f) \subset I$ the finite set of indices corresponding to the elements $b_{i}$ appearing in the expression of $f_{n}$. Following [14, Lemma 7.3], for any finite set $J \subset I$, we denote by $S(J) \subset I$ the finite set of indices corresponding to the generators $b^{i}$ satisfying $\left(\left[b \otimes b^{i}\right], \delta\left(b_{j}\right)\right) \neq 0$ for some $j \in J, b \in \mathfrak{b}_{+}$, and we define iteratively the finite sets $S_{n+1}(J)=S\left(S_{n}(J)\right), S_{0}(J)=J$. It follows immediately that for every $i \in I \backslash S_{n}\left(T_{n+1}(f)\right) b^{i} . f \in\left(N_{+}^{\vee}\right)_{n}$.

Similarly, one shows that the right $\mathfrak{g}_{\mathfrak{a}}-$ action on $N_{+}^{\vee}$ is equicontinuous by adapting the steps in $§ 3.9-\S 3.11$. This completes the proof of Proposition 3.7.

3.12. Coalgebra structure on $L_{-}$and $N_{+}$. Define the $\left(\mathfrak{g}_{\mathfrak{b}}, \mathfrak{g}_{\mathfrak{a}}\right)$-module maps

$$
i_{-}: L_{-} \rightarrow L_{-} \otimes L_{-} \quad \text { and } \quad i_{+}: N_{+} \rightarrow N_{+} \otimes N_{+}
$$

by mapping $\mathbf{1}_{\mp}$ to $\mathbf{1}_{\mp} \otimes \mathbf{1}_{\mp}$. Note that, under the identifications $L_{-} \simeq U \mathfrak{m}_{-}$and $N_{+} \simeq U \mathfrak{p}_{+}, i_{-}$and $i_{+}$correspond to the coproducts on $U \mathfrak{m}_{-}$and $U \mathfrak{p}_{+}$respectively.

Following [9, Prop. 1.2], we consider the invertible element $T \in U \mathfrak{g}_{\mathfrak{b}} \llbracket \hbar \rrbracket^{\otimes 2}$ satisfying the relations ${ }^{8}$

$$
\begin{aligned}
S^{\otimes 3}\left(\Phi_{\mathfrak{b}}{ }^{321}\right) \cdot(T \otimes 1) \cdot(\Delta \otimes 1)(T) & =(1 \otimes T)(1 \otimes \Delta)(T) \cdot \Phi_{\mathfrak{b}}, \\
T \Delta(S(a)) & =(S \otimes S)(\Delta(a)) T .
\end{aligned}
$$

Let $N_{+}^{\vee}$ be as before and $f, g \in N_{+}^{\vee}$. Consider the linear functional in $\operatorname{Hom}_{\mathrm{k}}\left(N_{+}, \mathrm{k}\right)$ defined by

$$
v \mapsto(f \otimes g)\left(T \cdot i_{+}(v)\right) .
$$

This functional is continuous, so it belongs to $N_{+}^{\vee}$ and induces a map $i_{+}^{\vee} \in \operatorname{Hom}_{\mathrm{k}}\left(N_{+}^{\vee} \otimes\right.$ $\left.N_{+}^{\vee}, N_{+}^{\vee}\right)$ by

$$
i_{+}^{\vee}(f \otimes g)(v)=(g \otimes f)\left(T \cdot i_{+}(v)\right) .
$$

\footnotetext{
${ }^{8} T$ is the element underlying the identification $(V \otimes W)^{*} \simeq W^{*} \otimes V^{*}$ in the category of representations of a quasi-Hopf algebra.
} 
For any $a \in \mathfrak{g}$, we have

$$
\begin{aligned}
i_{+}^{\vee}(a(f \otimes g))(v) & =(f \otimes g)\left((S \otimes S)(\Delta(a)) T \cdot i_{+}(v)\right) \\
& =(f \otimes g)\left(T \Delta(S(a)) \cdot i_{+}(v)\right) \\
& =i_{+}^{\vee}(f \otimes g)(S(a) \cdot v)=\left(a . i_{+}^{\vee}(f \otimes g)\right)(v)
\end{aligned}
$$

therefore $i_{+}^{\vee} \in \operatorname{Hom}_{\mathfrak{g}}\left(N_{+}^{\vee} \otimes N_{+}^{\vee}, N_{+}^{\vee}\right)$.

3.13. Set

$$
\Phi_{\mathfrak{a}}=\Phi\left(\hbar \Omega_{12}^{\mathfrak{a}}, \hbar \Omega_{23}^{\mathfrak{a}}\right) \quad \text { and } \quad \Phi_{\mathfrak{b}}=\Phi\left(\hbar \Omega_{12}^{\mathfrak{b}}, \hbar \Omega_{23}^{\mathfrak{b}}\right)
$$

and let $\mathcal{E}_{\left(\mathfrak{g}_{\mathfrak{b}}, \mathfrak{g}_{\mathfrak{a}}\right)}^{\Phi}$ be the Drinfeld category of equicontinuous $\left(\mathfrak{g}_{\mathfrak{b}}, \mathfrak{g}_{\mathfrak{a}}\right)$-bimodules, with commutativity and associativity constraints given respectively by

$$
(12) \circ \exp \left(\frac{\hbar}{2} \Omega^{\mathfrak{b}}\right) \circ \exp \left(-\frac{\hbar}{2} \Omega^{\mathfrak{a}}\right)^{\rho} \quad \text { and } \quad \Phi_{\mathfrak{b}} \circ\left(\Phi_{a}^{-1}\right)^{\rho}
$$

where $(-)^{\rho}$ denotes the right $\mathfrak{g}_{\mathfrak{a}}$-action.

The following shows that $L_{-}$and $N_{+}^{\vee}$ are coalgebra and algebra objects in $\mathcal{E}_{\left(\mathfrak{g}_{\mathfrak{b}}, \mathfrak{g}_{\mathfrak{a}}\right)}^{\Phi} \cdot$

Proposition. The following relations hold

(i) As morphisms $L_{-} \rightarrow L_{-} \otimes\left(L_{-} \otimes L_{-}\right)$,

$$
\Phi_{\mathfrak{b}}\left(i_{-} \otimes 1\right) i_{-}=\left(1 \otimes i_{-}\right) i_{-} .
$$

(ii) As morphisms $\left(N_{+}^{\vee} \otimes N_{+}^{\vee}\right) \otimes N_{+}^{\vee} \rightarrow N_{+}^{\vee}$

$$
i_{+}^{\vee}\left(1 \otimes i_{+}^{\vee}\right) \Phi_{\mathfrak{b}}=i_{+}^{\vee}\left(i_{+}^{\vee} \otimes 1\right) S^{\otimes 3}\left(\Phi_{\mathfrak{a}}^{-1}\right)^{\rho} .
$$

Proof. We begin by showing that

$$
\Phi_{\mathfrak{b}} \mathbf{1}_{-}^{\otimes 3}=\mathbf{1}_{-}^{\otimes 3} \quad \text { and } \quad \Phi_{\mathfrak{b}} \mathbf{1}_{+}^{\otimes 3}=\Phi_{\mathfrak{a}} \mathbf{1}_{+}^{\otimes 3} .
$$

To prove the first identity, it is enough to notice that, since $\mathfrak{b}_{+} \mathbf{1}_{-}=0$ and $\Omega=$ $\sum\left(a_{i} \otimes b^{i}+b^{i} \otimes a_{i}\right), \Omega_{i j}\left(\mathbf{1}_{-}^{\otimes 3}\right)=0$. Then $\Phi_{\mathfrak{b}} \mathbf{1}_{-}^{\otimes 3}=\mathbf{1}_{-}^{\otimes 3}$. To prove the second one, we notice that $\mathfrak{m}_{-} \mathbf{1}_{+}=0$ and that we can rewrite

$\Omega=\sum_{j \in I_{\mathfrak{a}}}\left(a_{j} \otimes b^{j}+b^{j} \otimes a_{j}\right)+\sum_{i \in I \backslash I_{\mathfrak{a}}}\left(a_{i} \otimes b^{i}+b^{i} \otimes a_{i}\right)=\Omega_{\mathfrak{a}}+\sum_{i \in I \backslash I_{\mathfrak{a}}}\left(a_{i} \otimes b^{i}+b^{i} \otimes a_{i}\right)$,

where $\left\{a_{j}\right\}_{j \in I_{\mathfrak{a}}}$ is a basis of $\mathfrak{a}_{-}$and $\left\{b^{j}\right\}_{j \in I_{\mathfrak{a}}}$ is the dual basis of $\mathfrak{a}_{+}$. Then

$$
\Omega_{i j} \mathbf{1}_{+}^{\otimes 3}=\Omega_{\mathfrak{a}, i j} \mathbf{1}_{+}^{\otimes 3}
$$

and, since for any element $x \in \mathfrak{g}_{\mathfrak{a}}$ the right and the left $\mathfrak{g}_{\mathfrak{a}}$-action coincide on $\mathbf{1}_{+}$, i.e., $x . \mathbf{1}_{+}=\mathbf{1}_{+} . x$, we have

$$
\Omega_{i j} \mathbf{1}_{+}^{\otimes 3}=\mathbf{1}_{+}^{\otimes 3} \Omega_{\mathfrak{a}, i j}
$$

and consequently $\Phi_{\mathfrak{b}} \mathbf{1}_{+}^{\otimes 3}=\Phi_{\mathfrak{a}} \mathbf{1}_{+}^{\otimes 3}$.

To prove (i), note that since the comultiplication in $U \mathfrak{m}_{-}$is coassociative, we have $\left(i_{-} \otimes 1\right) i_{-}=\left(1 \otimes i_{-}\right) i_{-}$. We therefore have to show that $\Phi_{\mathfrak{b}}\left(i_{-} \otimes 1\right) i_{-}=\left(1 \otimes i_{-}\right) i_{-}$. This is an obvious consequence of (3.1) and the fact that $L_{-}$is generated by $\mathbf{1}_{-}$. 
To prove (ii), consider $v \in N_{+}, f, g, h \in N_{+}^{\vee}$, then

$$
\begin{aligned}
i_{+}^{\vee} & \left(1 \otimes i_{+}^{\vee}\right)\left(\Phi_{\mathfrak{b}}(f \otimes g \otimes h)\right)(v) \\
& =(h \otimes g \otimes f)\left(\left(S^{\otimes 3}\left(\Phi_{\mathfrak{b}}{ }^{321}\right) \cdot(T \otimes 1) \cdot(\Delta \otimes 1)(T)\right) \cdot\left(i_{+} \otimes 1\right) i_{+}(v)\right) \\
& =(h \otimes g \otimes f)\left((1 \otimes T)(1 \otimes \Delta)(T) \cdot \Phi_{\mathfrak{b}}\left(i_{+} \otimes 1\right) i_{+}(v)\right) \\
& =(h \otimes g \otimes f)\left((1 \otimes T)(1 \otimes \Delta)(T)\left(1 \otimes i_{+}\right) i_{+}(v) \Phi_{\mathfrak{a}}\right) \\
& =\left(S^{\otimes 3}\left(\Phi_{\mathfrak{a}}\right)^{\rho}(h \otimes g \otimes f)\right)\left((1 \otimes T)(1 \otimes \Delta)(T)\left(1 \otimes i_{+}\right) i_{+}(v)\right) \\
& =i_{+}^{\vee}\left(i_{+}^{\vee} \otimes 1\right)\left(S^{\otimes 3}\left(\Phi_{\mathfrak{a}}^{321}\right)^{\rho}(f \otimes g \otimes h)\right)(v) \\
& =i_{+}^{\vee}\left(i_{+}^{\vee} \otimes 1\right) S^{\otimes 3}\left(\Phi_{\mathfrak{a}}^{-1}\right)^{\rho}(f \otimes g \otimes h)(v)
\end{aligned}
$$

and (ii) is proved.

3.14. The relative fiber functor. To any representation $V \in \mathcal{E}_{\mathfrak{g}_{\mathfrak{b}}}^{\Phi}$, we can associate the $\mathbf{k} \llbracket \hbar \rrbracket$-module

$$
F_{\mathfrak{a}, \mathfrak{b}}(V)=\operatorname{Hom}_{\mathcal{E}_{\mathfrak{g}_{\mathfrak{b}}}^{\Phi}}\left(L_{-}, N_{+}^{\vee} \otimes V\right),
$$

where $\operatorname{Hom}_{\mathcal{E}_{\mathfrak{g}_{\mathfrak{b}}}}$ is the set of continuous homomorphisms, equipped with the weak topology. The right $\mathfrak{g}_{\mathfrak{a}}$-action on $N_{+}^{*}$ endows $F_{\mathfrak{a}, \mathfrak{b}}(V)$ with the structure of a left $\mathfrak{g}_{\mathfrak{a}}-$ module.

Proposition. For any $V \in \mathcal{E}_{\mathfrak{g}_{\mathfrak{b}}}^{\Phi}, F_{\mathfrak{a}, \mathfrak{b}}(V)$ is isomorphic to $V$ as equicontinous $\mathfrak{g}_{\mathfrak{a}}-$ module. The isomorphism is given by

$$
\alpha_{V}: f \mapsto\left(\mathbf{1}_{+} \otimes 1\right) F_{\mathfrak{b}}\left(\mathbf{1}_{-}\right)
$$

for any $f \in \operatorname{Hom}_{\mathcal{E}_{\mathfrak{g}_{\mathfrak{b}}}}\left(L_{-}, N_{+}^{\vee} \otimes V\right)$. The assignment $V \mapsto F_{\mathfrak{a}, \mathfrak{b}}(V)$ extends to a functor $F_{\mathfrak{a}, \mathfrak{b}}: \mathcal{E}_{\mathfrak{g}_{\mathfrak{b}}}^{\Phi} \rightarrow \mathcal{E}_{\mathfrak{g}_{\mathfrak{a}}}^{\Phi}$.

The proof is carried out in $\S 3.15$ and $\S 3.16$.

3.15. By Frobenius reciprocity, we get an isomorphism

$$
\operatorname{Hom}_{\mathfrak{g}_{\mathfrak{b}}}\left(L_{-}, N_{+}^{\vee} \otimes V\right) \simeq \operatorname{Hom}_{\mathfrak{p}_{+}}\left(\mathrm{k}, N_{+}^{\vee} \otimes V\right) \simeq \operatorname{Hom}_{\mathrm{k}}(\mathrm{k}, V) \simeq V
$$

given by the map

$$
f \mapsto\left(\mathbf{1}_{+} \otimes 1\right) F_{\mathfrak{b}}\left(\mathbf{1}_{-}\right) .
$$

For $f \in F_{\mathfrak{a}, \mathfrak{b}}(V)$ and $x \in U \mathfrak{g}_{\mathfrak{a}}, x . f \in F_{\mathfrak{a}, \mathfrak{b}}(V)$ is defined by

$$
x . f=\left(S(x)^{\rho} \otimes \mathrm{id}\right) \circ f .
$$

For any $x \in U \mathfrak{g}_{\mathfrak{a}}$, we have

$$
\sum_{i, j} x_{i}^{(1)} f_{j} \otimes x_{i}^{(2)} v_{j}=\varepsilon(x) F_{\mathfrak{b}}\left(1_{-}\right),
$$

where $\Delta(x)=\sum_{i} x_{i}^{(1)} \otimes x_{i}^{(2)}$ and $F_{\mathfrak{b}}\left(\mathbf{1}_{-}\right)=\sum_{j} f_{j} \otimes v_{j}$. Using the identity

$$
1 \otimes x=\sum_{i}\left(S\left(x_{i}^{(1)}\right) \otimes 1\right) \cdot \Delta\left(x_{i}^{(2)}\right),
$$

we obtain

$$
(1 \otimes x) F_{\mathfrak{b}}\left(\mathbf{1}_{-}\right)=\sum_{i}\left(S\left(x_{i}^{(1)} \varepsilon\left(x_{i}^{(2)}\right)\right) \otimes 1\right) F_{\mathfrak{b}}\left(\mathbf{1}_{-}\right)=(S(x) \otimes 1) F_{\mathfrak{b}}\left(\mathbf{1}_{-}\right) .
$$


Finally, we have

$$
\begin{aligned}
x . \alpha_{V}(f) & =\left\langle\mathbf{1}_{+} \otimes \mathrm{id},(1 \otimes x) F_{\mathfrak{b}}\left(\mathbf{1}_{-}\right)\right\rangle \\
& =\left\langle\mathbf{1}_{+} \otimes \mathrm{id},(S(x) \otimes 1) F_{\mathfrak{b}}\left(\mathbf{1}_{-}\right)\right\rangle \\
& =\left\langle\mathbf{1}_{+} \otimes \mathrm{id},\left(S(x)^{\rho} \otimes 1\right) F_{\mathfrak{b}}\left(\mathbf{1}_{-}\right)\right\rangle=\alpha_{V}(x . f) .
\end{aligned}
$$

Therefore, $F_{\mathfrak{a}, \mathfrak{b}}(V)$ is isomorphic to $V$ as equicontinuous $\mathfrak{g}_{\mathfrak{a}}$-module.

3.16. For any continuous $\varphi \in \operatorname{Hom}_{\mathfrak{g}_{\mathfrak{b}}}\left(V, V^{\prime}\right)$, define a map $F_{\mathfrak{a}, \mathfrak{b}}(\varphi): F_{\mathfrak{a}, \mathfrak{b}}(V) \rightarrow$ $F_{\mathfrak{a}, \mathfrak{b}}\left(V^{\prime}\right)$ by

$$
F_{\mathfrak{a}, \mathfrak{b}}(\varphi): f \mapsto(\mathrm{id} \otimes \varphi) \circ f
$$

This map is continuous and for all $x \in \mathfrak{g}_{\mathfrak{a}}$

$$
F_{\mathfrak{a}, \mathfrak{b}}(\varphi)(x f)=\left(S(x)^{\rho} \otimes \varphi\right) \circ f=x F_{\mathfrak{a}, \mathfrak{b}}(\varphi)(f),
$$

therefore $F_{\mathfrak{a}, \mathfrak{b}}(\varphi) \in \operatorname{Hom}_{\mathfrak{g} \mathfrak{a}}\left(F_{\mathfrak{a}, \mathfrak{b}}(V), F_{\mathfrak{a}, \mathfrak{b}}\left(V^{\prime}\right)\right)$. Since the diagram

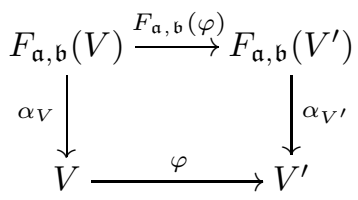

is commutative for all $\varphi \in \operatorname{Hom}_{\mathfrak{g}_{\mathfrak{b}}}\left(V, V^{\prime}\right)$, we have a well-defined functor

$$
F_{\mathfrak{a}, \mathfrak{b}}: \mathcal{E}_{\mathfrak{g}}^{\Phi_{\mathfrak{b}}} \rightarrow \mathcal{E}_{\mathfrak{g}_{\mathfrak{a}}}^{\Phi}
$$

which is naturally isomorphic to the pullback functor induced by the inclusion $i_{\mathfrak{a}}: \mathfrak{g}_{\mathfrak{a}} \hookrightarrow \mathfrak{g}_{\mathfrak{b}}$ via the natural transformation

$$
\alpha_{V}: F_{\mathfrak{a}, \mathfrak{b}}(V) \simeq i_{\mathfrak{a}}^{*} V
$$

This completes the proof of Proposition 3.14.

3.17. Tensor structure on $F_{\mathfrak{a}, \mathfrak{b}}$. Denote the tensor product in the categories $\mathcal{E}_{\mathfrak{g}_{\mathfrak{b}}}^{\Phi}$, $\mathcal{E}_{\mathfrak{g}_{\mathfrak{a}}}^{\Phi}$ by $\otimes$, and let $B_{1234}$ and $B_{1234}^{\prime}$ be the associativity constraints

$$
B_{1234}:\left(V_{1} \otimes V_{2}\right) \otimes\left(V_{3} \otimes V_{4}\right) \rightarrow V_{1} \otimes\left(\left(V_{2} \otimes V_{3}\right) \otimes V_{4}\right)
$$

and

$$
B_{1234}^{\prime}:\left(V_{1} \otimes V_{2}\right) \otimes\left(V_{3} \otimes V_{4}\right) \rightarrow\left(V_{1} \otimes\left(V_{2} \otimes V_{3}\right)\right) \otimes V_{4} .
$$

For any $v \in F_{\mathfrak{a}, \mathfrak{b}}(V), w \in F_{\mathfrak{a}, \mathfrak{b}}(W)$, define $J_{V W}(v \otimes w) \in F_{\mathfrak{a}, \mathfrak{b}}(V \otimes W)$ by the composition

$$
\begin{aligned}
& L_{-} \stackrel{i_{-}}{\longrightarrow} L_{-} \otimes L_{-} \stackrel{v \otimes w}{\longrightarrow}\left(N_{+}^{\vee} \otimes V\right) \otimes\left(N_{+}^{\vee} \otimes W\right) \stackrel{B}{\longrightarrow} N_{+}^{\vee} \otimes\left(\left(V \otimes N_{+}^{\vee}\right) \otimes W\right) \\
& \stackrel{\beta_{23}^{-1}}{\longrightarrow} N_{+}^{\vee} \otimes\left(\left(N_{+}^{\vee} \otimes V\right) \otimes W\right) \stackrel{B^{\prime}}{\longrightarrow}\left(N_{+}^{\vee} \otimes N_{+}^{\vee}\right) \otimes(V \otimes W) \stackrel{i_{+}^{\vee} \otimes 1}{\longrightarrow} N_{+}^{\vee} \otimes(V \otimes W) .
\end{aligned}
$$

The map $J_{V W}(v \otimes w)$ is clearly a continuous $\mathfrak{g}_{\mathfrak{b}}$-morphism from $L_{-}$to $N_{+}^{\vee} \otimes(V \otimes W)$, so one gets a well-defined map

$$
J_{V W}: F_{\mathfrak{a}, \mathfrak{b}}(V) \otimes F_{\mathfrak{a}, \mathfrak{b}}(W) \rightarrow F_{\mathfrak{a}, \mathfrak{b}}(V \otimes W) .
$$

Proposition. The maps $J_{V W}$ are isomorphisms of $\mathfrak{g}_{\mathfrak{a}}-$ modules, and define a tensor structure on the functor $F_{\mathfrak{a}, \mathfrak{b}}$.

The proof of Proposition 3.17 is carried out in $\S 3.18-\S 3.21$. 
3.18. The map $J_{V W}$ is compatible with the $\mathfrak{g}_{\mathfrak{a}}$-action. Indeed, $i_{+}^{\vee}$ is a morphism of right $\mathfrak{g}_{\mathfrak{a}}-$ modules and, for any $x \in \mathfrak{g}_{\mathfrak{a}}$,

$$
\begin{aligned}
x J_{V W}(v \otimes w) & =\left(S(x)^{\rho} \otimes \mathrm{id}\right)\left(i_{+}^{\vee} \otimes \mathrm{id} \otimes \mathrm{id}\right) \widetilde{A}(v \otimes w) i_{-} \\
& =\left(i_{+}^{\vee} \otimes \mathrm{id} \otimes \mathrm{id}\right)\left(\Delta(S(x))^{\rho}\right)_{12} \widetilde{A}(v \otimes w) i_{-} \\
& \left.=\left(i_{+}^{\vee} \otimes \mathrm{id} \otimes \mathrm{id}\right) \widetilde{A}((S \otimes S)(\Delta(x)))^{\rho}\right)_{13}(v \otimes w) i_{-}=J_{V W}(x(v \otimes w))
\end{aligned}
$$

where $\widetilde{A}=A^{\prime} \beta_{32}^{-1} A$.

$J_{V W}$ is an isomorphism, since it is an isomorphism modulo $\hbar$. Indeed,

$$
J_{V W}(v \otimes w) \equiv\left(i_{+}^{*} \otimes 1\right)(1 \otimes s \otimes 1)(v \otimes w) i_{-} \quad \bmod \hbar .
$$

To prove that $J_{V W}$ define a tensor structure on $F_{\mathfrak{a}, \mathfrak{b}}$, we need to show that, for any $V_{1}, V_{2}, V_{3} \in \mathcal{E}_{\mathfrak{g}_{\mathfrak{b}}}^{\Phi}$ the following diagram is commutative

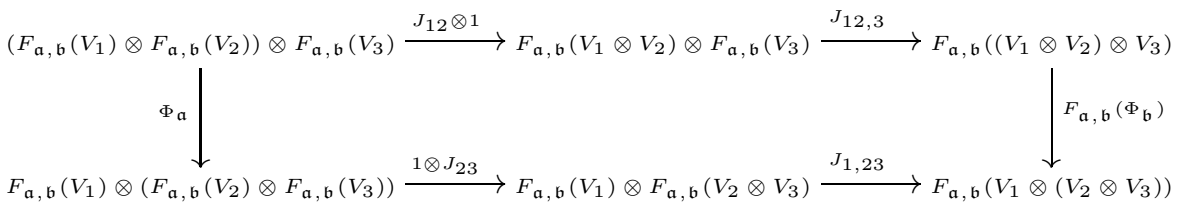

where $J_{i j}$ denotes the map $J_{V_{i}, V_{j}}$ and $J_{i j, k}$ the map $J_{V_{i} \otimes V_{j}, V_{k}}$.

3.19. For any $v_{i} \in F_{\mathfrak{a}, \mathfrak{b}}\left(V_{i}\right), i=1,2,3$, the map $F_{\mathfrak{a}, \mathfrak{b}}\left(\Phi_{\mathfrak{b}}\right) J_{12,3} J_{12} \otimes 1\left(v_{1} \otimes v_{2} \otimes v_{3}\right)$ is given by the composition

$$
\begin{aligned}
\left(1 \otimes \Phi_{\mathfrak{b}}\right)\left(i_{+}^{*} \otimes 1^{\otimes 3}\right) A_{4}\left(1 \otimes \beta_{1 \otimes 2, N_{+}^{\vee}} \otimes 1\right) A_{3}\left(\left(i_{+}^{*} \otimes 1\right) \otimes 1^{\otimes 3}\right)\left(A_{2} \otimes 1 \otimes 1\right) \\
\cdot\left(1 \otimes \beta_{N_{+}^{\vee}, 1} \otimes 1^{\otimes 3}\right)\left(A_{1} \otimes 1 \otimes 1\right)\left(v_{1} \otimes v_{2} \otimes v_{3}\right)\left(i_{-} \otimes 1\right) i_{-},
\end{aligned}
$$

where

$$
\begin{array}{ll}
A_{1}=B_{N_{+}^{\vee}, 1, N_{+}^{\vee}, 2}, & A_{3}=B_{N_{+}^{\vee}, 1 \otimes 2, N_{+}^{\vee}, 3}, \\
A_{2}=B_{N_{+}^{\vee}, N_{+}^{\vee}, 1,2}, & A_{4}=B_{N_{+}^{\vee}, N_{+}^{\vee}, 1 \otimes 2,3}^{1} .
\end{array}
$$

By functoriality of associativity and commutativity isomorphisms, we have

$$
A_{3}\left(i_{+}^{*} \otimes 1^{\otimes 4}\right)=\left(i_{+}^{*} \otimes 1^{\otimes 4}\right) A_{5},
$$

where $A_{5}=B_{N_{+}^{\vee} \otimes N_{+}^{\vee}, 12, N_{+}^{\vee}, 3}$,

$$
\left(1 \otimes \beta_{12, N_{+}^{\vee}} \otimes 1\right)\left(i_{+}^{*} \otimes 1^{\otimes 4}\right)=\left(i_{+}^{*} \otimes 1^{\otimes 4}\right)\left(1^{\otimes 2} \otimes \beta_{12, N_{+}^{\vee}} \otimes 1^{\otimes 2}\right)
$$

and

$$
A_{4}\left(i_{+}^{*} \otimes 1^{\otimes 4}\right)=\left(i_{+}^{*} \otimes 1^{\otimes 4}\right) A_{6}
$$

where $A_{6}=B_{N_{+}^{\vee} \otimes N_{+}^{\vee}, N_{+}^{\vee}, 1 \otimes 2,3}^{-1}$. Finally, we have

$$
\begin{aligned}
F_{\mathfrak{a}, \mathfrak{b}}\left(\Phi_{\mathfrak{b}}\right) & J_{12,3}\left(J_{12} \otimes 1\right)\left(v_{1} \otimes v_{2} \otimes v_{3}\right) \\
& =\left(1^{\otimes 3} \otimes \Phi_{\mathfrak{b} 123}\right)\left(\left(i_{+}^{*}\left(i_{+}^{*} \otimes 1\right)\right) \otimes 1^{\otimes 3}\right) A\left(v_{1} \otimes v_{2} \otimes v_{3}\right)\left(i_{-} \otimes 1\right) i_{-},
\end{aligned}
$$

where

$$
A=A_{6}\left(1^{\otimes 2} \otimes \beta_{1 \otimes 2, N_{+}^{\vee}} \otimes 1^{\otimes 2}\right) A_{5}\left(A_{2} \otimes 1^{\otimes 2}\right)\left(1 \otimes \beta_{N_{+}^{\vee}, 1} \otimes 1^{\otimes 3}\right)\left(A_{1} \otimes 1 \otimes 1\right) .
$$


3.20. On the other hand, $J_{1,2 \otimes 3}\left(1 \otimes J_{23}\right) \Phi_{\mathfrak{a}}\left(v_{1} \otimes v_{2} \otimes v_{3}\right)$ corresponds to the composition

$$
\begin{aligned}
\left(i_{+}^{*} \otimes 1^{\otimes 3}\right) A_{4}^{\prime}\left(1 \otimes \beta_{N_{+}^{\vee}, 1} \otimes 1^{\otimes 2}\right) A_{3}^{\prime}\left(1^{\otimes 2} \otimes i_{+}^{*} \otimes 1^{\otimes 2}\right)\left(1 \otimes 1 \otimes A_{2}^{\prime}\right) \\
\left(1^{\otimes 3} \otimes \beta_{2, N_{+}^{\vee}} \otimes 1\right)\left(1 \otimes 1 \otimes A_{1}^{\prime}\right) \Phi_{\mathfrak{a}}\left(v_{1} \otimes v_{2} \otimes v_{3}\right)\left(1 \otimes i_{-}\right) i_{-},
\end{aligned}
$$

where

$$
\begin{array}{ll}
A_{1}^{\prime}=B_{N_{-}^{\vee}, 2, N_{+}^{\vee}, 3}, & A_{3}^{\prime}=B_{N_{-}^{\vee}, 1, N_{+}^{\vee}, 2 \otimes 3}, \\
A_{2}^{\prime}=B_{N_{+}^{\vee}, N_{+}^{\vee}, 2,3}, & A_{4}^{\prime}=B_{N_{+}^{\vee}, N_{+}^{\vee}, 1,2 \otimes 3}^{\vee} .
\end{array}
$$

By functoriality of associativity and commutativity isomorphisms, we have

$$
A_{3}^{\prime}\left(1^{\otimes 2} \otimes i_{+}^{*} \otimes 1^{\otimes 2}\right)=\left(1^{\otimes 2} \otimes i_{+}^{*} \otimes 1^{\otimes 2}\right) A_{5}^{\prime},
$$

where $A_{5}^{\prime}=B_{N_{+}^{\vee}, 1, N_{+}^{\vee} \otimes N_{+}^{\vee}, 2 \otimes 3}$,

$$
\left(1 \otimes \beta_{1, N_{+}^{\vee}} \otimes 1^{\otimes 2}\right)\left(1^{\otimes 2} \otimes i_{+}^{*} \otimes 1^{\otimes 2}\right)=\left(1 \otimes i_{+}^{*} \otimes 1^{\otimes 3}\right)\left(1 \otimes \beta_{1, N_{+}^{\vee} \otimes N_{+}^{\vee}} \otimes 1^{\otimes 2}\right),
$$

and

$$
A_{4}^{\prime}\left(1 \otimes i_{+}^{*} \otimes 1^{\otimes 3}\right)=\left(1 \otimes i_{+}^{*} \otimes 1^{\otimes 3}\right) A_{6}^{\prime},
$$

where $A_{6}^{\prime}=B_{N_{+}^{\vee}, N_{+}^{\vee} \otimes N_{+}^{\vee}, 1,2 \otimes 3}^{-1}$. Thus,

$$
\begin{aligned}
J_{1,23}\left(1 \otimes J_{23}\right) & \Phi_{\mathfrak{a}}\left(v_{1} \otimes v_{2} \otimes v_{3}\right) \\
& =\left(i_{+}^{*} \otimes 1^{\otimes 3}\right)\left(\left(1 \otimes i_{+}^{*}\right) \otimes 1^{\otimes 3}\right) B \Phi_{\mathfrak{a}}\left(v_{1} \otimes v_{2} \otimes v_{3}\right)\left(1 \otimes i_{-}\right) i_{-},
\end{aligned}
$$

where

$$
B=A_{6}^{\prime}\left(1 \otimes \beta_{1, N_{+}^{\vee} \otimes N_{+}^{\vee}} \otimes 1^{\otimes 2}\right) A_{5}^{\prime}\left(1^{\otimes 2} \otimes A_{2}^{\prime}\right)\left(1^{\otimes 3} \otimes \beta_{2, N_{+}^{\vee}} \otimes 1\right)\left(1 \otimes 1 \otimes A_{1}^{\prime}\right) .
$$

3.21. Comparing (3.2) and (3.3), we see that it suffices to show that the outer arrows of the following form a commutative diagram.

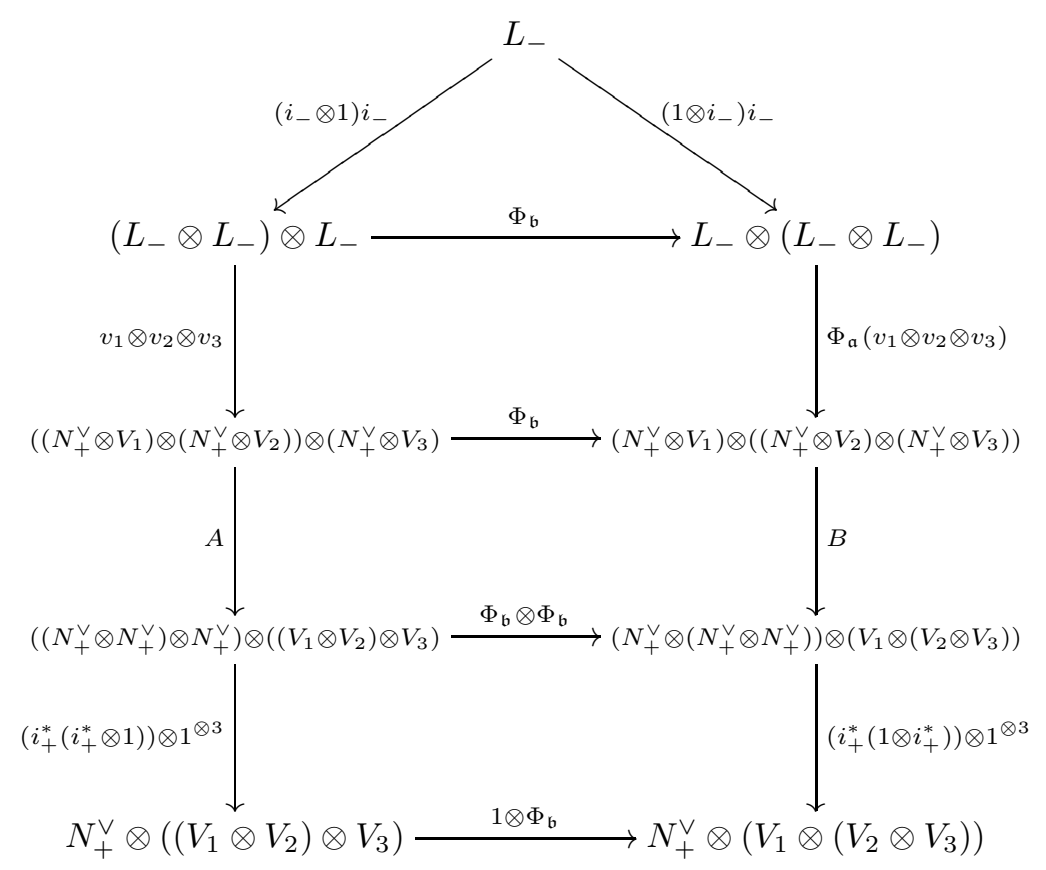


Using the pentagon and the hexagon axiom, one shows that $\left(\Phi_{\mathfrak{b}} \otimes \Phi_{\mathfrak{b}}\right) A=B \Phi_{\mathfrak{b}}$. We have to show that

$$
F_{\mathfrak{a}, \mathfrak{b}}\left(\Phi_{\mathfrak{b}}\right) J_{12,3}\left(J_{12} \otimes 1\right)\left(v_{1} \otimes v_{2} \otimes v_{3}\right)=J_{1,23}\left(1 \otimes J_{23}\right) \Phi_{\mathfrak{a}}\left(v_{1} \otimes v_{2} \otimes v_{3}\right)
$$

in $\operatorname{Hom}_{\mathfrak{g}}\left(L_{-}, N_{+}^{\vee} \otimes\left(V_{1} \otimes\left(V_{2} \otimes V_{3}\right)\right)\right)$ :

$$
\begin{aligned}
J_{1,23}\left(\mathrm{id} \otimes J_{23}\right) \Phi_{\mathfrak{a}}\left(v_{1} \otimes v_{2} \otimes v_{3}\right) \\
=\left(i_{+}^{\vee}\left(\mathrm{id} \otimes i_{+}^{\vee}\right) \otimes \mathrm{id}^{\otimes 3}\right) B \Phi_{\mathfrak{a}}\left(v_{1} \otimes v_{2} \otimes v_{3}\right)\left(\mathrm{id} \otimes i_{-}\right) i_{-} \\
=\left(i_{+}^{\vee}\left(\mathrm{id} \otimes i_{+}^{\vee}\right) \otimes \mathrm{id}^{\otimes 3}\right) B \Phi_{\mathfrak{a}}\left(v_{1} \otimes v_{2} \otimes v_{3}\right) \Phi_{\mathfrak{b}}\left(i_{-} \otimes \mathrm{id}\right) i_{-} \\
\quad=\left(i_{+}^{\vee}\left(\mathrm{id} \otimes i_{+}^{\vee}\right) \otimes \mathrm{id}^{\otimes 3}\right) B \Phi_{\mathfrak{b}} \Phi_{\mathfrak{a}}\left(v_{1} \otimes v_{2} \otimes v_{3}\right)\left(i_{-} \otimes \mathrm{id}\right) i_{-} \\
\quad=\left(i_{+}^{\vee}\left(\mathrm{id} \otimes i_{+}^{\vee}\right) \Phi_{\mathfrak{b}} \otimes \Phi_{\mathfrak{b}}\right) A \Phi_{\mathfrak{a}}\left(v_{1} \otimes v_{2} \otimes v_{3}\right)\left(i_{-} \otimes \mathrm{id}\right) i_{-} \\
=\left(i_{+}^{\vee}\left(\mathrm{id} \otimes i_{+}^{\vee}\right) \Phi_{\mathfrak{b}} \otimes \Phi_{\mathfrak{b}}\right)\left(S^{\otimes 3}\left(\Phi_{\mathfrak{a}}\right)^{\rho} \otimes \mathrm{id}^{\otimes 3}\right) A\left(v_{1} \otimes v_{2} \otimes v_{3}\right)\left(i_{-} \otimes \mathrm{id}\right) i_{-} \\
=\left(i_{+}^{\vee}\left(\mathrm{id} \otimes i_{+}^{\vee}\right) \Phi_{\mathfrak{b}} S^{\otimes 3}\left(\Phi_{\mathfrak{a}}\right)^{\rho} \otimes \Phi_{\mathfrak{b}}\right) A\left(v_{1} \otimes v_{2} \otimes v_{3}\right)\left(i_{-} \otimes \mathrm{id}\right) i_{-} \\
=\left(i_{+}^{\vee}\left(i_{+}^{\vee} \otimes \mathrm{id}\right) \otimes \Phi_{\mathfrak{b}}\right) A\left(v_{1} \otimes v_{2} \otimes v_{3}\right)\left(i_{-} \otimes \mathrm{id}\right) i_{-} \\
\quad=F_{\mathfrak{a}, \mathfrak{b}}\left(\Phi_{\mathfrak{b}}\right) J_{12,3}\left(J_{12} \otimes \mathrm{id}\right)\left(v_{1} \otimes v_{2} \otimes v_{3}\right),
\end{aligned}
$$

where the second and seventh equalities follow from Proposition 3.13, the fifth one from the definition of the $\mathfrak{g}_{\mathfrak{a}}$-action on the modules $F_{\mathfrak{a}, \mathfrak{b}}\left(V_{i}\right)$ and the others from functoriality of the associator $\Phi_{\mathfrak{b}}$. We shall henceforth denote the tensor structure on $F_{\mathfrak{a}, \mathfrak{b}}$ by $J_{\mathfrak{a}, \mathfrak{b}}$.

3.22. Infinitesimal of relative twist $J_{\mathfrak{a}, \mathfrak{b}}$. The following is a straightforward extension of the computation of the 1-jet of the Etingof-Kazhdan twist.

Proposition. Under the natural identification $\alpha_{V}: F_{\mathfrak{a}, \mathfrak{b}}(V) \rightarrow V$, the relative twist $J_{\mathfrak{a}, \mathfrak{b}}$ satisfies

$$
\alpha_{V \otimes W} \circ J_{\mathfrak{a}, \mathfrak{b}} \circ\left(\alpha_{V}^{-1} \otimes \alpha_{W}^{-1}\right) \equiv \mathrm{id}^{\otimes 2}+\frac{\hbar}{2}\left(r_{\mathfrak{b}}+i^{\otimes 2}\left(r_{\mathfrak{a}}^{21}\right)\right) \quad \bmod \hbar^{2}
$$

in $\operatorname{End}(V \otimes W)$, where $r_{\mathfrak{b}}, r_{\mathfrak{a}}$ are the canonical elements in $\mathfrak{b} \otimes \mathfrak{b}^{*}, \mathfrak{a} \otimes \mathfrak{a}^{*}$ respectively.

Proof. For $v \in V, w \in W$, let

$$
\alpha_{V}^{-1}(v)\left(1_{-}\right)=\sum f_{i} \otimes v_{i} \quad \alpha_{W}^{-1}(w)\left(1_{-}\right)=\sum g_{j} \otimes w_{j}
$$

in $\left(N_{+}^{\vee} \otimes V\right)^{\mathfrak{p}_{+}}$and $\left(N_{+}^{\vee} \otimes W\right)^{\mathfrak{p}_{+}}$respectively. Then we observe

$$
\left\langle\left(1_{+} \otimes 1\right)^{\otimes 2}, \bar{\Omega}_{23} \sum_{i, j} f_{i} \otimes v_{i} \otimes g_{j} \otimes w_{j}\right\rangle=-\bar{r}(v \otimes w)
$$

and

$$
\left\langle\left(1_{+} \otimes 1\right)^{\otimes 2}, i^{\otimes 2}\left(\Omega_{\mathfrak{a}}\right)_{23} \sum_{i, j} f_{i} \otimes v_{i} \otimes g_{j} \otimes w_{j}\right\rangle=-i^{\otimes 2}\left(\Omega_{\mathfrak{a}}\right)(v \otimes w),
$$

where $\Omega=\bar{\Omega}+i^{\otimes 2}\left(\Omega_{\mathfrak{a}}\right)$. Together with the fact that $\Phi_{\mathfrak{a}}, \Phi_{\mathfrak{b}}=1^{\otimes 3} \bmod \hbar^{2}$, we obtain

$$
\alpha_{V \otimes W} \circ J_{\mathfrak{a}, \mathfrak{b}} \circ\left(\alpha_{V}^{-1} \otimes \alpha_{W}^{-1}\right)(v \otimes w) \equiv v \otimes w+\frac{\hbar}{2}\left(r_{\mathfrak{b}}+i^{\otimes 2}\left(r_{\mathfrak{a}}\right)\right)(v \otimes w) \quad \bmod \hbar^{2} .
$$

The computation of the 1 -jet of $J_{\mathfrak{a}, \mathfrak{b}}$ shows, in particular, that for $\mathfrak{a}=\mathfrak{b}$ the twist $J_{\mathfrak{b}, \mathfrak{b}}$ is not trivial. However, by adapting the arguments of [14, Prop. 9.7] and [5, Prop. 3.10], one observes that the functor $F_{\mathfrak{b}, \mathfrak{b}}$ is in fact tensor equivalent to 
the Etingof-Kazhdan functor $F_{\mathfrak{b}}$ equipped with the trivial tensor structure, and it is therefore tensor equivalent to the identity functor on $\mathcal{E}_{\mathfrak{g}}^{\Phi} \simeq \mathrm{DY}_{\mathfrak{b}_{-}}^{\Phi}$.

This complete the proof of Theorem 3.1.

\section{Quantisation of Verma modules}

In this section, we construct a natural transformation $v_{\mathfrak{a}, \mathfrak{b}}$ making the following diagram commute

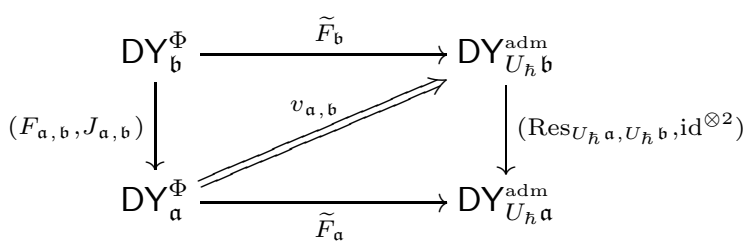

where $\left(F_{\mathfrak{a}, \mathfrak{b}}, J_{\mathfrak{a}, \mathfrak{b}}\right)$ is the tensor functor constructed in Section 3 , and $\operatorname{Res}_{U_{\hbar} \mathfrak{b}, U_{\hbar} \mathfrak{a}}$ is the tensor functor induced by the split embedding $U_{\hbar} \mathfrak{a} \hookrightarrow U_{\hbar} \mathfrak{b}$. The proof relies on the construction of a quantum restriction functor $F_{\mathfrak{a}, \mathfrak{b}}^{\hbar}$ using a quantum version $L_{-}^{\hbar}, N_{+}^{\hbar}$ of the relative Verma modules $L_{-}, N_{+}^{\vee}$. The natural transformation $v_{\mathfrak{a}, \mathfrak{b}}$ is then obtained under the assumption that the Etingof-Kazhdan quantisation of $L_{-}, N_{+}^{\vee}$ is given by their their quantum counterparts $L_{-}^{\hbar}, N_{+}^{\hbar}$, a fact which will proved in Section $\S 7$. We begin in $\S 4.1-\S 4.5$ by discussing the analogous, but simpler, quantisation of the Verma modules $M_{-}, M_{+}^{\vee}$. The quantum Verma modules $L_{-}^{\hbar}, N_{+}^{\hbar}$ are studied in $\S 4.10-\$ 4.12$. Their definition relies on the Radford biproduct associated on a split pair of Hopf algebras, which is reviewed in $\S 4.6-\S 4.9$

4.1. The quantum Verma module $M_{B}$. The Verma module $M_{-}$defined in $\S 2.13$ has a natural counterpart in the category of Drinfeld-Yetter modules over a Hopf algebra $(B, m, \iota, \Delta, \epsilon, S)$ over k. Following $[15, \S 2.3]$, define the Drinfeld-Yetter $B$ module $M_{B}$ to be the k-module $B$, with action $\pi_{-}$given by the multiplication $m$. Since $M_{B}$ is a free $B$-module of rank one, with cyclic vector 1 , the coaction $\pi_{-}^{*}$ on $M_{B}$ is uniquely determined by its compatibility (2.7) with the action, together with the requirement that $\pi_{-}^{*} 1=1 \otimes 1$. $\pi_{-}^{*}$ is easily seen to be given by

$$
\pi_{-}^{*}=m^{21} \otimes \mathrm{id} \circ S^{-1} \otimes(23) \circ \Delta^{(3)} .
$$

which coincides with the adjoint coaction of $B$ on itself.

$M_{B}$ satisfies the following universal property. For every $V \in \mathrm{DY}_{B}$,

$$
\operatorname{Hom}_{B}^{B}\left(M_{B}, V\right) \simeq \operatorname{Hom}^{B}(\mathrm{k}, V) .
$$

The isomorphism (4.2) is easily described. In one direction, for any Drinfeld-Yetter morphism $g: M_{B} \rightarrow V$, one sets $\phi(g)=g \circ \iota$. Since $\iota$ commutes with the coaction, $\phi(g)$ is a morphism of $B$-comodules. Conversely, for any morphism of comodules $f: \mathrm{k} \rightarrow V$, one defines a map $\psi(f): M_{B} \rightarrow V$ by

$$
\psi(f)=\pi_{V} \circ \mathrm{id} \otimes f
$$

where $\pi_{V}$ denotes the action on $V$. It is easy to see that $\psi(f)$ is a morphism of Drinfeld-Yetter modules and $\phi, \psi$ are inverse of each other.

It follows from (4.2) that $M_{B}$ is naturally endowed with a cocommutative coalgebra structure in $\mathrm{DY}_{B}$, induced by the coproduct of $B$. 
4.2. The quantum Verma module $\widehat{M_{B}^{\vee}}$. The module $\widehat{M_{B}^{\vee}}$ is defined from $M_{B}$ by reversing all arrows and exchanging product with coproduct and action with coaction. Namely, set $\widehat{M_{B}^{\vee}}=B$ with coaction $\pi_{+}^{*}=\Delta^{21}$, and action

$$
\pi_{+}=m^{(3)} \circ(23) \circ\left(\mathrm{id} \otimes S^{-1} \otimes \mathrm{id}\right) \circ \Delta^{21} \otimes \mathrm{id},
$$

given by the adjoint action of $B$ on itself. The module $\widehat{M_{B}^{\vee}}=B$ satisfies the following universal property. For any $V \in \mathrm{DY}_{B}$,

$$
\operatorname{Hom}_{B}^{B}\left(V, \widehat{M_{B}^{\vee}}\right) \simeq \operatorname{Hom}_{B}(V, \mathrm{k})
$$

The isomorphism is described as follows. For any Drinfeld-Yetter morphism $g$ : $V \rightarrow \widehat{M_{B}^{\vee}}$, one sets $\phi(g)=\epsilon \circ g$. Since the counit commutes with the action of $B, \phi(g)$ is a morphism of $B$-modules. Conversely, for any morphism of modules $f: V \rightarrow$ k, one defines a map $\psi(f): V \rightarrow \widehat{M_{B}^{\vee}}$ by

$$
\psi(f)=\mathrm{id} \otimes f \circ \pi_{V}^{*},
$$

where $\pi_{V}^{*}$ denotes the coaction on $V$. It is easy to see that $\psi(f)$ is a morphism of Drinfeld-Yetter modules and $\phi, \psi$ are inverse of each other. Moreover, it follows from (4.4) that $\widehat{M_{B}^{\mathrm{V}}}$ is naturally endowed with a commutative algebra structure in $\mathrm{DY}_{B}$, induced by the product of $B$.

As pointed out in $\S 2.18$, for any finite-dimensional Hopf algebra $B$, the category of Drinfeld-Yetter $B$-modules is equivalent to that of modules over the quantum double $D B=B \otimes B^{\circ}$ (see also Appendix A). Under this equivalence, the module $M_{B}$ corresponds to the $D B$-module induced by the inclusion $B^{\circ} \subset D B$, while $\widehat{M_{B}^{\vee}}$ corresponds to the coinduced $D B$-module corresponding to the inclusion $B \subset D B$.

4.3. Admissibility of $M_{B}$. If $B$ is a quantised enveloping algebra, it is natural to restrict attention to the category of admissible Drinfeld-Yetter module defined in $\S 2.20$, i.e., those whose coaction factors through the Hopf subalgebra $B^{\prime} \subset B$.

Proposition. Let $B$ be a QUE. The adjoint coaction (4.1) of $B$ on itself factors through $B^{\prime} \otimes B$. In particular, the Drinfeld-Yetter module $M_{B}$ is admissible.

Proof. By Proposition 2.21, it is enough to check that $\pi_{-}^{*}$ satisfies (2.9). One has

$$
\begin{aligned}
m^{21} \otimes \mathrm{id} \circ S^{-1} \otimes(23) \circ\left(\mathrm{id} \otimes \Delta^{21} \circ \Delta\right) & =\left(m^{21} \circ S^{-1} \otimes \mathrm{id} \circ \Delta\right) \otimes \mathrm{id} \circ \Delta \\
& =(\iota \circ \epsilon) \otimes \mathrm{id} \circ \Delta=\iota \otimes \mathrm{id} \\
& =(\iota \circ \epsilon) \otimes \mathrm{id} \circ \pi_{-}^{*} .
\end{aligned}
$$

It follows that

$$
(\mathrm{id}-\iota \circ \epsilon) \otimes \mathrm{id} \circ \pi_{-}^{*}=m^{21} \otimes \mathrm{id} \circ S^{-1} \otimes(23) \circ\left(\mathrm{id} \otimes\left(\Delta-\Delta^{21}\right) \circ \Delta\right),
$$

which is divisible by $\hbar$ since $\Delta-\Delta^{21}$ is. Therefore $\pi_{-}^{*}$ satisfies (2.9) and $M_{B}$ is admissible.

4.4. The quantum Verma module $M_{B}^{\vee}$. It is easy to check that the module $\widehat{M_{B}^{\vee}}$ is not admissible. This suggests modifying the definition of $\widehat{M_{B}^{\vee}}$ in order to obtain a solution to the universal property (4.4) in the category of admissible DrinfeldYetter modules.

Proposition. Let $B$ be a $Q U E$.

(i) The adjoint action (4.3) $\pi_{+}$of $B$ onto itself preserves $B^{\prime} \subset B$. 
(ii) The $\mathrm{K}$-module $M_{B}^{\vee}=B^{\prime}$, with action given by $\pi_{+}$and coaction by $\pi_{+}^{*}=$ $\Delta^{21}$ is an admissible Drinfeld-Yetter B-module.

(iii) $M_{B}^{\vee}$ satisfies the universal property (4.4) in the category $\mathrm{DY}_{B}^{\mathrm{adm}}$.

Proof. (ii) and (iii) follows from (i) and the previous discussion. (i) Set $\delta^{(n)}=$ $(\text { id }-\iota \circ \varepsilon)^{\otimes n} \circ \Delta^{(n)}$. We have to show that, for any $n \geq 1, \operatorname{Im}\left(\delta^{(n)} \circ \pi_{+}\right) \subset \hbar^{n} B^{\otimes n}$. We proceed by induction. For $n=1$,

$$
\delta^{(1)} \circ \pi_{+}=\pi_{+} \circ \text { id } \otimes \delta^{(1)} .
$$

Namely, for every $b \in B, b^{\prime} \in B^{\prime}$, one has

$$
\begin{aligned}
b_{2} b^{\prime} S^{-1}\left(b_{1}\right)-\varepsilon\left(b_{2} b S^{-1} b_{1}\right) & =b_{2} b^{\prime} S^{-1} b_{1}-\varepsilon(b) \varepsilon\left(b^{\prime}\right) \\
& =b_{2} b S^{-1} b_{1}-b_{2} S^{-1} b_{1} \varepsilon\left(b^{\prime}\right) \\
& =b_{2} b S^{-1} b_{1}-b_{2} \varepsilon\left(b^{\prime}\right) S^{-1} b_{1}=\pi_{+} \circ \mathrm{id} \otimes \delta^{(1)} .
\end{aligned}
$$

Assume now that $\operatorname{Im}\left(\delta^{(n)} \circ \pi_{+}\right) \subset \hbar^{n} B^{\otimes n}$ and set $\Delta-\Delta^{21}=\hbar \Xi$. Then

$$
\begin{gathered}
\delta^{(n+1)} \circ \pi_{+}=\mathrm{id} \otimes \delta^{(1)} \circ \mathrm{id} \otimes m^{(3)} \circ(1432) \circ \mathrm{id} \otimes\left(\delta^{(n)} \circ \pi_{+}\right) \otimes \mathrm{id}^{\otimes 2} \\
\circ S^{-1} \otimes \mathrm{id} \otimes(34) \otimes \mathrm{id} \circ \Delta^{(3)} \otimes \Delta \\
=\left(\delta^{(n)} \circ \pi_{+}\right) \otimes\left(\delta^{(1)} \circ \pi_{+}\right) \circ(23) \circ \Delta \otimes \Delta \\
+\hbar \mathrm{id} \otimes \delta^{(1)} \circ \mathrm{id} \otimes m^{(3)} \circ(1432) \circ \mathrm{id} \otimes\left(\delta^{(n)} \circ \pi_{+}\right) \otimes \mathrm{id}^{\otimes 2} \\
\circ S^{-1} \otimes \mathrm{id} \otimes(34) \otimes \mathrm{id} \circ \Xi \otimes \mathrm{id}^{\otimes 2} \circ \Delta \otimes \Delta .
\end{gathered}
$$

It follows by induction that $\operatorname{Im}\left(\delta^{(n+1)} \circ \pi_{+}\right) \subset \hbar^{n+1} B^{\otimes n+1}$.

4.5. Quantisation of $M_{-}$and $M_{+}^{\vee}$. Let now $\mathfrak{b}$ be a Lie bialgebra, and $U_{\hbar} \mathfrak{b}$ its quantisation. We denote by $M_{-}^{\hbar}$ and $\left(M_{+}^{\vee}\right)^{\hbar}$ the admissible Drinfeld-Yetter $U_{\hbar} \mathfrak{b}-$ modules $M_{U_{\hbar} \mathfrak{b}}$ and $M_{U_{\hbar} \mathfrak{b}}^{\vee}$, respectively.

Proposition. The following holds in the category $\mathrm{DY}_{U_{\hbar} \mathfrak{b}}^{\mathrm{adm}}$

(a) $\widetilde{F}_{\mathfrak{b}}\left(M_{-}\right) \simeq M_{-}^{\hbar}$ as coalgebras,

(b) $\widetilde{F}_{\mathfrak{b}}\left(M_{+}^{\vee}\right) \simeq\left(M_{+}^{\vee}\right)^{\hbar}$ as algebras.

Proof. The Hopf algebra $U_{\hbar} \mathfrak{b}$ is constructed on the vector space $F_{\mathfrak{b}}\left(M_{-}\right)$with unit element $u \in F_{\mathfrak{b}}\left(M_{-}\right)$defined by $u\left(1_{-}\right)=\epsilon_{+} \otimes 1_{-}$, where $\epsilon_{+} \in M_{+}^{\vee}$ is given by $\epsilon_{+}\left(x 1_{+}\right)=\epsilon(x)$ for any $x \in U \mathfrak{b}_{+}$. Consequently, the action of $U_{\hbar} \mathfrak{b}$ on $u \in F_{\mathfrak{b}}\left(M_{-}\right)$ is free, as multiplication with the unit element. The coaction of $U_{\hbar} \mathfrak{b}$ on $F_{\mathfrak{b}}\left(M_{-}\right)$is defined using the $\mathcal{R}$-matrix associated to the braided tensor functor $F$, i.e.,

$$
\pi_{M_{-}}^{*}: F_{\mathfrak{b}}\left(M_{-}\right) \rightarrow F_{\mathfrak{b}}\left(M_{-}\right) \otimes F_{\mathfrak{b}}\left(M_{-}\right), \quad \pi^{*}(x)=\mathcal{R}(u \otimes x),
$$

where $x \in F_{\mathfrak{b}}\left(M_{-}\right)$and $\mathcal{R}_{V W} \in \operatorname{End}_{U_{\hbar} \mathfrak{g}}\left(F_{\mathfrak{b}}(V) \otimes F_{\mathfrak{b}}(W)\right)$ is given by $\mathcal{R}_{V W}=$ $\sigma J_{W V}^{-1} F_{\mathfrak{b}}\left(\beta_{V W}\right) J_{V W},\left\{J_{V, W}\right\}_{V, W \in \mathrm{DY}}$ being the tensor structure on $F$. It is easy to show that $\left.J(u \otimes u)\right|_{1_{-}}=\epsilon_{+} \otimes 1_{-} \otimes 1_{-}$, and, since $\Omega\left(1_{-} \otimes 1_{-}\right)=0$, we have

$$
\pi_{M_{-}}^{*}=\mathcal{R}(u \otimes u)=u \otimes u .
$$

By construction the coalgebra structure on $F_{\mathfrak{b}}\left(M_{-}\right)$coincides with that on $U_{\hbar} \mathfrak{b}$. This proves $(a)$. 
The module $M_{+}^{\vee}$ satisfies the following universal property: for any $V \in \mathrm{DY}_{\mathfrak{b}}^{\Phi}$, one has

$$
\operatorname{Hom}_{\mathfrak{b}}^{\mathfrak{b}}\left(V, M_{+}^{\vee}\right) \simeq \operatorname{Hom}_{\mathfrak{b}}(V, \mathrm{k}) .
$$

Since $\widetilde{F}_{\mathfrak{b}}$ is an equivalence of categories,

$$
\operatorname{Hom}_{U_{\hbar} \mathfrak{b}}^{U_{\hbar} \mathfrak{b}}\left(\widetilde{F}_{\mathfrak{b}}(V), \widetilde{F}_{\mathfrak{b}}\left(M_{+}^{\vee}\right)\right) \simeq \operatorname{Hom}_{\mathfrak{b}}^{\mathfrak{b}}\left(V, M_{+}^{\vee}\right) \simeq \operatorname{Hom}_{\mathfrak{b}}(V, \mathrm{k})
$$

Using the isomorphism $\alpha_{V}: F_{\mathfrak{b}}(V) \rightarrow V$ defined by

$$
\alpha_{V}(f)=\left\langle f\left(1_{-}\right), 1_{+} \otimes \mathrm{id}\right\rangle,
$$

we obtain a map $\operatorname{Hom}_{\mathfrak{b}}(V, \mathrm{~K}) \rightarrow \operatorname{Hom}_{\mathfrak{K}}\left(F_{\mathfrak{b}}(V), \mathrm{K}\right)$. For any $x \in U \mathfrak{b}$ let $\psi_{x}: M_{-} \rightarrow$ $M_{+}^{\vee} \otimes M_{-}$be the morphism defined by $\psi_{x}\left(1_{-}\right)=\epsilon_{+} \otimes x 1_{-}$. It is clear that, if $f\left(1_{-}\right)=f_{(1)} \otimes f_{(2)}$ in sumless Swedler's notation,

$$
\begin{aligned}
\alpha_{V}\left(\psi_{x} . f\right) & =\left\langle\left(i_{+}^{\vee} \otimes \mathrm{id}\right) \Phi^{-1}(\mathrm{id} \otimes f)\left(\epsilon_{+} \otimes x .1_{-}\right), 1_{+} \otimes \mathrm{id}\right\rangle \\
& =\left\langle\Phi^{-1}\left(\epsilon_{+} \otimes \mathrm{id} \otimes \mathrm{id}\right)(\mathrm{id} \otimes \Delta(x))\left(\mathrm{id} \otimes f_{(1)} \otimes f_{(2)}\right),(T \otimes \mathrm{id})\left(1_{+} \otimes 1_{+} \mathrm{id}\right)\right\rangle \\
& =\left\langle\Delta(x)\left(f_{(1)} \otimes f_{(2)}\right), 1_{+} \otimes \mathrm{id}\right\rangle \\
& =\left\langle f_{(1)}, 1_{+}\right\rangle x \cdot f_{(2)} \\
& =x \cdot \alpha_{V}(f),
\end{aligned}
$$

using the fact that $(\epsilon \otimes 1 \otimes 1)(\Phi)=1^{\otimes 2}$ and $(\epsilon \otimes 1)(T)=1$. So, clearly, if $\phi \in \operatorname{Hom}_{\mathfrak{b}}(V, \mathrm{k})$, then $\phi \circ \alpha_{V} \in \operatorname{Hom}_{U_{\hbar} \mathfrak{b}}\left(F_{\mathfrak{b}}(V), \mathrm{k} \llbracket \hbar \rrbracket\right)$ and we obtain an isomorphism of Drinfeld-Yetter modules $F_{\mathfrak{b}}\left(M_{+}^{\vee}\right) \simeq\left(M_{+}^{\vee}\right)^{\hbar}$. By universal property, this is compatible with the algebra structures and $(b)$ is proved.

4.6. Split pair of Hopf algebras. We briefly recall the characterisation of a Hopf algebra with a projection given by Radford [26]. Let $A \stackrel{i}{\rightarrow} B \stackrel{p}{\rightarrow} A, p \circ i=\mathrm{id}_{A}$, be a split pair of Hopf algebras. One can describe the kernel of the projection $p$ and obtain $B$ as a semidirect product of Hopf algebras. Let $\pi \in \operatorname{End}(B)$ be the idempotent $\pi=i \circ p$, and define $\Pi \in \operatorname{End}(B)$ by

$$
\Pi=m \circ \mathrm{id} \otimes(S \circ \pi) \circ \Delta
$$

(equivalently, $\Pi=\mathrm{id} *(S \circ \pi)$ in the convolution algebra $\operatorname{End}(B))$.

It is easy to show, using standard graphical calculus, that $\Pi$ is an idempotent such that

$$
\Pi \circ \pi=\iota \circ \epsilon=\pi \circ \Pi \text {. }
$$

Moreover, if $L=\Pi(B) \subseteq B$, then $B \simeq L \otimes A$ via the mutually inverse maps

$$
B \underset{m}{\stackrel{\Pi \otimes \pi \circ \Delta}{\rightleftarrows}} L \otimes A
$$

The following result describes the structure of $L$.

\section{Theorem.}

(i) The following identities hold:

$$
\Pi \circ m=m^{(3)} \circ(23) \circ \mathrm{id} \otimes(S \circ \pi) \otimes \mathrm{id} \circ \Delta \otimes \Pi
$$

and

$$
\Pi \circ m \circ \mathrm{id} \otimes \pi=\Pi \otimes \epsilon .
$$

Moreover, $L$ is a $B$-module with respect to the adjoint action of $B$

$$
\pi_{L}=m^{(3)} \circ \mathrm{id} \otimes \mathrm{id} \otimes(S \circ \pi) \circ(23) \circ \Delta \otimes \Pi .
$$


(ii) The following identity holds

$$
\mathrm{id} \otimes \pi \circ \Delta \circ \Pi=\Pi \otimes \mathrm{id}
$$

and it characterises $L$. Moreover, $L$ is a subalgebra in $B$.

(iii) The following identities hold:

$$
\Delta \circ \Pi=m \otimes \mathrm{id} \circ(23) \circ \mathrm{id} \otimes \Pi \otimes(S \circ \pi) \circ \Delta^{(3)}
$$

and

$$
\mathrm{id} \otimes \pi \circ \Delta \circ \Pi=\Pi \otimes \eta .
$$

Moreover, the map

$$
\pi_{L}^{*}=m^{21} \otimes \mathrm{id} \circ S^{-1} \otimes(23) \circ \Delta^{(3)} \circ \Pi
$$

defines a $B$-comodule structure on $L$.

(iv) $\left(L, \pi_{L}, \pi_{L}^{*}\right)$ is a Drinfeld-Yetter $B$-module (and therefore a Drinfeld-Yetter $A$-module by restriction), and satisfies the universal property

$$
\operatorname{Hom}_{B}^{B}(L, V) \simeq \operatorname{Hom}_{A}^{B}(\mathrm{k}, V)
$$

for every $V \in \mathrm{DY}_{B}$.

(v) $L$ is a cocommutative coalgebra object in $\mathrm{DY}_{B}$ with comultiplication

$$
\Delta_{L}=\Pi \otimes \Pi \circ \Delta \circ \Pi \text {. }
$$

(vi) $L$ is a Hopf algebra in $\mathrm{DY}_{A}$.

(vii) If $(B, A)$ is a split pair of $Q U E s$, then $L$ is an admissible Drinfeld-Yetter $B$-module.

Proof. The identities (4.7), (4.8), (4.9), (4.10), and (4.11) are verified by standard graphical calculus for Hopf algebras (e.g. [17, Lecture 8]).

(i) The identity (4.7) implies that the adjoint action of $B$ on itself preserves $L$, and therefore the module structure on $L$ is well-defined.

(ii) One shows that

$$
\mathrm{id} \otimes \pi \circ \Delta \circ(m \circ \Pi \otimes \Pi)=(m \circ \Pi \otimes \Pi) \otimes \iota .
$$

Since (4.9) characterises $L$, it follows that $L$ closed under multiplication in $B$.

(iii) It follows from (4.10) that the coaction (4.12) takes values in $B \otimes L$. One then shows directly that $\pi_{L}^{*}$ is a coaction.

(iv) The compatibility between $\pi_{L}$ and $\pi_{L}^{*}$ is verified by direct inspection. Then, we observe that the action of $A$ and the coaction of $B$ on the unit element $u_{L} \in L$ are trivial. In particular, by restriction to $u_{L} \in L$, we get a map

$$
\operatorname{Hom}_{B}^{B}(L, V) \rightarrow \operatorname{Hom}_{A}^{B}(\mathrm{k}, V)
$$

for every $V \in \mathrm{DY}_{B}$, whose inverse is given by composition with the action of $B$ restricted to $L$.

$(v)$ By (4.13), the coalgebra structure on $L$ is uniquely determined by the condition $\Delta_{L}\left(u_{L} \otimes u_{L}\right)=u_{L} \otimes u_{L}$. Since the action of $B$ on $u_{L}$ coincides with the projection $\Pi$, one recovers (4.14). Finally, since the $R$-matrix preserves $u_{L} \otimes u_{L}$, i.e.,

$$
\pi_{L} \otimes \mathrm{id} \circ(23) \circ \mathrm{id} \otimes \pi_{L}^{*}\left(u_{L} \otimes u_{L}\right)=u_{L} \otimes u_{L}
$$

it follows that $\Delta_{L}=(12) \circ R \circ \Delta$, i.e., $L$ is a cocommutative coalgebra in the category $\mathrm{DY}_{B}$. 
(vi) The compatibility between the product and the coproduct on $L$ is a straightforward computation. It follows from (4.8) that the map $\Pi$ is a morphism of $A$ modules with respect to the adjoint action of $A$ on $B$. Since the multiplication in $B$ commutes with the action of $A$ and $L$ is a subalgebra in $B, L$ is a $A$-module algebra. Similarly one shows that $L$ is a $A$-comodule coalgebra.

(vii) The admissibility of $L$ follows as in Proposition 4.3.

4.7. Radford biproduct. The object $L \otimes A$ is naturally endowed with a Hopf algebra structure, induced by the identification $B \simeq L \otimes A$ (4.6). The description of such structure relies exclusively on that of $A$ and $L$ (as Hopf algebra in DY $A$ ).

Conversely, given a Hopf algebra $A$ in Vect and a Hopf algebra $L$ in $\mathrm{DY}_{A}$, the tensor product $L \otimes A$ is endowed with a Hopf algebra structure, called Radford biproduct (further studied by Majid under the name of bosonisation [24]).

Namely, let $\left(A, m_{A}, \eta_{A}, \Delta_{A}, \varepsilon_{A}, S_{A}\right)$ be a Hopf algebra in Vect and $\left(L, m_{L}, \eta_{L}, \Delta_{L}\right.$, $\left.\varepsilon_{L}, S_{L}\right)$ a Hopf algebra in $\mathrm{DY}_{A}$, with Drinfeld-Yetter structure $\left(L, \pi_{L}, \pi_{L}^{*}\right)$. The Radford biproduct $L \star A$ is the Hopf algebra defined on $L \otimes A$ with operations

$$
\begin{aligned}
m_{L \star A} & =m_{L} \otimes m_{A} \circ \mathrm{id} \otimes \pi_{L} \otimes \mathrm{id}^{\otimes 2} \circ(34) \circ \mathrm{id} \otimes \Delta_{A} \otimes \mathrm{id}^{\otimes 2}, \\
\Delta_{L \star A} & =\mathrm{id} \otimes m_{A} \otimes \mathrm{id}^{\otimes 2} \circ(34) \circ \mathrm{id} \otimes \pi_{L}^{*} \otimes \mathrm{id}^{\otimes 2} \circ \Delta_{L} \otimes \Delta_{A}, \\
S_{L \star A} & =\pi_{L} \otimes \mathrm{id}^{\otimes 2} \circ(23) \circ \Delta_{L} \otimes \mathrm{id} \circ S_{L} \otimes S_{A} \circ m_{A} \otimes \mathrm{id} \circ(23) \circ \pi_{L}^{*} \otimes \mathrm{id} .
\end{aligned}
$$

unit $\eta_{L} \otimes \eta_{A}$ and counit $\varepsilon_{L} \otimes \varepsilon_{A}$. It is easy to see that $L \star A$ contains $L$ as a subalgebra and $A$ as a Hopf subalgebra. In fact,

$$
A \stackrel{\eta_{L} \otimes \mathrm{id}}{\longrightarrow} L \star A \stackrel{\epsilon_{L} \otimes \mathrm{id}}{\longrightarrow} A
$$

is a split pair of Hopf algebras.

4.8. Radford biproduct and Drinfeld-Yetter modules. Since $L$ is a Hopf algebra in $\mathrm{DY}_{A}$, a Drinfeld-Yetter module over the Radford biproduct $L \star A$ is conveniently described as a Drinfeld-Yetter module over $L$ in the category $\mathrm{DY}_{A}$.

Proposition. There is a canonical equivalence of braided tensor categories

$$
\mathrm{DY}_{L \star A} \simeq \mathrm{DY}_{L, A},
$$

where $\mathrm{DY}_{L, A}$ denotes the category of Drinfeld-Yetter L-modules in $\mathrm{DY}_{A}$. Namely,

(i) Any $\left(V, \pi_{L, V}, \pi_{L, V}^{*}, \pi_{A, V}, \pi_{A, V}^{*}\right) \in \mathrm{DY}_{L, A}$ is naturally a Drinfeld-Yetter $L \star A-$ module with action and coaction

$$
\pi_{L \star A, V}=\pi_{L, V} \circ \mathrm{id} \otimes \pi_{A, V} \quad \text { and } \quad \pi_{L \star A, V}^{*}=\mathrm{id} \otimes \pi_{A, V}^{*} \circ \pi_{L, V}^{*} .
$$

(ii) Conversely, any $\left(V, \pi_{L \star A, V}, \pi_{L \star A, V}^{*}\right) \in \mathrm{DY}_{L \star A}$ has, by restriction to $L$ and $A$, a structure of Drinfeld-Yetter L-module in $\mathrm{DY}_{A}$ with

$$
\begin{array}{ll}
\pi_{A, V}=\pi_{L \star A, V} \circ \iota_{L} \otimes \mathrm{id}_{A} \otimes \mathrm{id}_{V} & \pi_{A, V}^{*}=\varepsilon_{L} \otimes \operatorname{id}_{A} \otimes \mathrm{id}_{V} \circ \pi_{L \star A, V}^{*} \\
\quad \text { and } & \\
\pi_{L, V}=\pi_{L \star A, V} \circ \mathrm{id}_{L} \otimes \iota_{A} \otimes \mathrm{id}_{V} & \pi_{L, V}^{*}=\mathrm{id}_{L} \otimes \varepsilon_{A} \otimes \mathrm{id}_{V} \circ \pi_{L \star A, V}^{*} .
\end{array}
$$

Proof. First, one shows that the assignments in $(i)$ and $(i i)$ define an equivalence between the tensor categories $\operatorname{Rep}(L \star A)$ of $(L \star A)$-modules and $\operatorname{Rep}_{A}(L)$ of $L^{-}$ modules in $\operatorname{Rep}(A)$ (cf. [28, Sec. 1.7, Prop. 2]). This restricts to an equivalence 
of braided tensor categories at the level of Drinfeld centers (cf. [19, XIII.4]), which are equivalent to the categories $\mathrm{DY}_{L \star A}$ and $\mathrm{DY}_{L, A}$, respectively.

4.9. Admissible Drinfeld-Yetter modules. In Section §5, we will study the relation between the quantisation of a split pair of Lie bialgebras and the Radford biproduct. In particular, we will need the following result.

Proposition. Let $(B, A)$ be a split pair of QUEs. The equivalence given by Proposition 4.8 restricts to an equivalence of admissible Drinfeld-Yetter modules

$$
\mathrm{DY}_{L, A}^{\mathrm{adm}} \simeq \mathrm{DY}_{L \star A}^{\mathrm{adm}} .
$$

This reduces to proving the following lemma.

Lemma. Let $A$ be a QUE, $L$ a Hopf algebra object in $\mathrm{DY}_{A}^{\mathrm{adm}}$ and $B=L \star A$ the corresponding Radford biproduct. Then $B^{\prime}=L^{\prime} \star A^{\prime}$, where, for any (possibly braided) Hopf algebra $H$ over $\mathrm{k} \llbracket \hbar$, we set $p_{H}=\operatorname{id}_{H}-\iota_{H} \circ \epsilon_{H}, d_{H}^{(n)}=p_{H}^{\otimes n} \circ \Delta_{H}^{(n)}$, and

$$
H^{\prime}=\left\{x \in H \mid d_{H}^{(n)}(x) \in \hbar^{n} H^{\otimes n} \forall n\right\} .
$$

Proof. The Hopf algebras $\left(B^{\prime}, A^{\prime}\right)$ form a split pair, and, by Radford theorem, there is an isomorphism of Hopf algebras $B^{\prime} \simeq \widetilde{L} \star A^{\prime}$, where $\widetilde{L}$ is the image of the idempotent (4.5) corresponding to $B^{\prime}$. It is easy to show that

$$
\widetilde{L}=\left\{x \in B^{\prime} \mid \operatorname{id} \otimes \pi_{A} \circ \Delta_{B}(x)=x \otimes 1\right\}=L \cap B^{\prime} .
$$

We want to show that

$$
L^{\prime}=\left\{x \in L \mid d_{L}^{(n)}(x) \in \hbar^{n} L^{\otimes n}\right\}=L \cap B^{\prime},
$$

from which it follows that $B^{\prime}=L^{\prime} \star A^{\prime}$.

It follows immediately from the description of the Radford biproduct in $\S 4.6$ that $\Delta_{L}^{(n)}=\Pi^{\otimes n} \circ \Delta_{B}^{(n)} \circ \Pi$, and therefore

$$
\begin{aligned}
d_{L}^{(n)} & =p_{L}^{\otimes n} \circ \Delta_{L}^{(n)} \\
& =\left(\left(\mathrm{id}-\epsilon_{L}\right) \circ \Pi\right)^{\otimes n} \circ \Delta_{B} \circ \Pi \\
& =\Pi^{\otimes n} \circ\left(\mathrm{id}-\epsilon_{B}\right)^{\otimes n} \circ \Delta_{B} \circ \Pi \\
& =\Pi^{\otimes n} \circ d_{B}^{(n)} \circ \Pi .
\end{aligned}
$$

Hence, $L \cap B^{\prime} \subset L^{\prime}$. Conversely, since $\Delta_{B}(L) \subset B \otimes L$ and, under the identification $B \simeq L \star A, p_{B}=p_{L} \otimes \mathrm{id}+\epsilon_{L} \otimes p_{A}$, one has

$$
\begin{aligned}
d_{B}^{(2)} \circ \Pi & =p_{B}^{\otimes 2} \circ \Delta_{B} \circ \Pi \\
& =p_{B}^{\otimes 2} \circ \mathrm{id} \otimes \pi_{L}^{*} \circ \Delta_{L} \\
& =p_{L} \otimes \mathrm{id} \otimes p_{L} \circ \mathrm{id} \otimes \pi_{L}^{*} \circ \Delta_{L}+\epsilon_{L} \otimes p_{A} \otimes p_{L} \circ \mathrm{id} \otimes \pi_{L}^{*} \circ \Delta_{L} \\
& =\mathrm{id} \otimes \pi_{L}^{*} \circ d_{L}^{(2)}+d_{A}^{(1)} \otimes \mathrm{id} \circ \pi_{L}^{*} \circ d_{L}^{(1)},
\end{aligned}
$$

where $\pi_{L}^{*}: L \rightarrow A^{\prime} \otimes L$ denotes the admissible coaction of $A$ on $L$. It is then easy to see, by induction on $n$, that

$$
d_{B}^{(n)} \circ \Pi=\sum_{k=0}^{n-1} \gamma_{n}^{(n-k)} \circ d_{L}^{(k)},
$$


where $\gamma_{n}^{(n-k)}: L^{\otimes k} \rightarrow(L \otimes A)^{\otimes k-1} \otimes L$ is divisible by $\hbar^{n-k}$. Namely, we have

$$
\begin{aligned}
d_{B}^{(n)} \circ \Pi & =p_{B} \otimes\left(d_{B}^{(n-1)} \circ \Pi\right) \circ \Delta_{B} \\
& =\sum_{k=0}^{n-1} p_{B} \otimes \mathrm{id}^{\otimes n-1} \circ \mathrm{id} \otimes \gamma_{n-1}^{(n-k-1)} \circ \mathrm{id} \otimes d_{L}^{(k)} \circ \mathrm{id} \otimes \pi_{L}^{*} \circ \Delta_{L} \\
& =\sum_{k=0}^{n-1} \mathrm{id} \otimes \gamma_{n-1}^{(n-k-1)} \circ \mathrm{id} \otimes \pi_{L}^{* \otimes n-1} \circ d_{L}^{(k+1)}+d_{A}^{(1)} \otimes \gamma_{n-1}^{(n-k-1)} \circ \pi_{L}^{* \otimes n-1} \circ d_{L}^{(k)} .
\end{aligned}
$$

Since the coaction of $A$ on $L$ is admissible, one can proceed by setting for every $k=0,1, \ldots, n-1$

$$
\gamma_{n}^{(n-k)}=\mathrm{id} \otimes \gamma_{n-1}^{(n-k)} \circ \mathrm{id} \otimes \pi_{L^{\otimes n-1}}^{*}+d_{A}^{(1)} \otimes \gamma_{n-1}^{(n-k-1)} \circ \pi_{L^{\otimes n-1}}^{*} .
$$

Finally, relation (4.15) implies $L^{\prime} \subset L \cap B^{\prime}$ and proves the result.

4.10. The quantum relative Verma modules. The relative Verma modules $L_{-}, N_{+}^{\vee}$, constructed in Section $\S 3$ for any split pair of Lie bialgebras, have natural quantum counterparts, given by the relative analogues of the quantum Verma modules $M_{B}, M_{B}^{\vee}$ with respect to a split pair of Hopf algebras. In the following, we focus on the case of as split pair of QUEs.

We have seen in $\S 4.1$ that the Drinfeld-Yetter structures of $M_{B}$ and $M_{B}^{\vee}$ are tailored around the universal properties (4.2), (4.4). The same principle applies to the construction of the quantum relative Verma modules.

Let $A \stackrel{i_{\hbar}}{\rightarrow} B \stackrel{p_{\hbar}}{\rightarrow} A, p_{\hbar} \circ i_{\hbar}=\mathrm{id}_{A}$, be a split pair of QUEs, and $\mathfrak{a} \stackrel{i}{\rightarrow} \mathfrak{b} \stackrel{p}{\rightarrow} \mathfrak{a}$ the corresponding split pair of Lie bialgebras. We denote by $L_{B, A}$ the Drinfeld-Yetter $B$-module $L$ constructed in $\S 4.6$ on the image of the idempotent (4.5)

$$
\Pi=m_{B} \circ \mathrm{id} \otimes\left(S_{B} \circ \pi_{\hbar}\right) \circ \Delta_{B} .
$$

It follows from $\S 4.6$ that $L_{B, A} \subset B$ is a subalgebra and $B \simeq L_{B, A} \star A$. In particular, for $A=\mathrm{K}$, it is easy to check that the modules $L_{B, \mathrm{~K}}$ and $M_{B}$ coincide.

The construction of $N_{B, A}^{\vee}$ is similar to that of $M_{B}^{\vee}$ described in $\S 4.4$. As a Drinfeld-Yetter $B$-modules it is realised on $B^{\prime} \otimes A^{*}$ with coaction $\pi_{N_{B, A}^{\vee}}^{*}=\Delta_{B}^{21} \otimes$ id and action

$$
\pi_{N_{B, A}^{\vee}}=m_{B}^{(3)} \otimes \triangleleft_{A} \circ \mathrm{id}^{\otimes 2} \otimes S_{B}^{-1} \otimes \mathrm{id}^{\otimes 2} \circ(24) \circ \mathrm{id} \otimes p_{\hbar} \otimes \mathrm{id}^{\otimes 3} \circ \Delta_{B}^{(3)} \otimes \mathrm{id}^{\otimes 2},
$$

where $\triangleleft_{A}$ denotes the coadjoint action of $A$ on $A^{*}$. The structure of Drinfeld-Yetter $A^{\text {op }}$-module is given by the same formulae applied on the right to $A^{\prime} \otimes A^{*} \subset B^{\prime} \otimes A^{*}$. For $A=\mathrm{K}$, the modules $M_{B}^{\vee}$ and $N_{B, \mathrm{~K}}^{\vee}$ coincide.

\subsection{Properties of relative Verma modules.}

\section{Proposition.}

(i) $L_{B, A}, N_{B, A}^{\vee}$ are admissible Drinfeld-Yetter B-modules and satisfy the universal properties

$$
\begin{aligned}
& \operatorname{Hom}_{B}^{B}\left(L_{B, A}, \mathcal{V}\right) \simeq \operatorname{Hom}_{A}^{B}(K, \mathcal{V}), \\
& \operatorname{Hom}_{B}^{B}\left(\mathcal{V}, N_{B, A}^{\vee}\right) \simeq \operatorname{Hom}_{L_{B, A}}(\mathcal{V}, K),
\end{aligned}
$$

for any admissible $\mathcal{V} \in \mathrm{DY}_{B}^{\mathrm{adm}}$. Moreover, $L_{B, A}, N_{B, A}^{\vee}$ have, respectively, natural structures of coalgebra and algebra objects in $\mathrm{DY}_{B}^{\mathrm{adm}}$. 
(ii) $N_{B, A}^{\vee}$ satisfies the universal property (equivalent to (4.18))

$$
\operatorname{Hom}_{B}^{B}\left(\mathcal{V}, N_{B, A}^{\vee}\right) \simeq \operatorname{Hom}_{A}^{B}\left(\mathcal{V}, p_{\hbar}^{*} N_{A, A}^{\vee}\right)
$$

where $B$ acts on $N_{A, A}^{\vee}$ by projection to $A$.

(iii) As Drinfeld-Yetter $(\mathfrak{b}, \mathfrak{a})$-bimodules, the semiclassical limit of $L_{B, A}, N_{B, A}^{\vee}$ are, respectively, $L_{-}$and $N_{+}^{\vee}$.

Proof. (i) The universal properties (4.17), (4.18) generalise those of $M_{B}$ and $M_{B}^{\vee}$ described in $\S 4.1$. Specifically, we observe that the action of $A$ and the coaction of $B$ on the element $1 \in L_{B, A}$ are trivial. In particular, by restriction to $1 \in L_{B, A}$, we get a map

$$
\operatorname{Hom}_{B}^{B}\left(L_{B, A}, \mathcal{V}\right) \rightarrow \operatorname{Hom}_{A}^{B}(K, \mathcal{V})
$$

whose inverse is given by composition with the action of $B$ restricted to $L_{B, A}$. This proves (4.17) and implies that $L_{B, A}$ is endowed with a coalgebra structure in $\mathrm{DY}_{B}$.

The proof of (4.18) goes along the same lines. There is a map

$$
\operatorname{Hom}_{B}^{B}\left(\mathcal{V}, N_{B, A}^{\vee}\right) \rightarrow \operatorname{Hom}_{L_{B, A}}(\mathcal{V}, K),
$$

obtained by composition with the counits of $B^{\prime}$ and $A^{*}$, whose inverse is given by

$$
f \mapsto \mathrm{id} \otimes \mathrm{id} \otimes f \circ \pi_{V}^{*} \otimes \mathrm{id} \otimes \pi_{V} \circ R_{A} \otimes \mathrm{id},
$$

where $R_{A}$ denotes the universal $R$-matrix of $A$. It follows in particular that $N_{B, A}^{\vee}$ is naturally endowed with an algebra structure in $\mathrm{DY}_{B}$.

(ii) The isomorphism (4.19) is proved similarly. The map

$$
\operatorname{Hom}_{B}^{B}\left(\mathcal{V}, N_{B, A}^{\vee}\right) \rightarrow \operatorname{Hom}_{B}^{A}\left(\mathcal{V}, p_{\hbar}^{*} N_{A, A}^{\vee}\right)
$$

is obtained by composition with the canonical projection $N_{B, A}^{\vee} \rightarrow N_{A, A}^{\vee}$ induced by $B^{\prime} \rightarrow A^{\prime}$. The inverse map is obtained by precomposing with the coaction of $\mathcal{V}$ projected onto $L_{B, A}^{\prime}$. It is easy to check from Lemma 4.9 that this is well-defined and gives the desired isomorphism.

(iii) The idempotent $\Pi$ reduces modulo $\hbar$ to the canonical projection $U \mathfrak{b} \rightarrow U \mathfrak{m}$, where $\mathfrak{m}=\operatorname{ker}(p)$. It follows that $\mathrm{SC}\left(L_{B, A}\right) \simeq U \mathfrak{m}$. One checks that, under the canonical identification $L_{-} \simeq U \mathfrak{m} \simeq \mathrm{SC}\left(L_{B, A}\right)$, the $B$-action on $L_{B, A}$ reduces to the standard $\mathfrak{b}$-action on $L_{-}$. Since the coaction on $L_{-}$is uniquely determined by the condition $\pi_{L_{-}}^{*}\left(u_{L}\right)=0$, one concludes that $\mathrm{SC}\left(\pi_{L_{B, A}}^{*}\right)=\pi_{L_{-}}^{*}$, and therefore $\mathrm{SC}\left(L_{B, A}\right) \simeq L_{-}$in $\mathrm{DY}_{\mathfrak{b}}$.

The proof for $N_{B, A}^{\vee}$ goes as follows. It is clear that $\mathrm{SC}\left(N_{B, A}^{\vee}\right) \simeq N_{+}^{\vee}$ as $\mathfrak{b}_{-}$ comodules. One then observes that the maps

$$
\operatorname{Hom}_{\mathfrak{b}}^{\mathfrak{b}}\left(V, \mathrm{SC}\left(N_{B, A}^{\vee}\right)\right) \underset{\phi}{\stackrel{\psi}{\rightleftarrows}} \operatorname{Hom}_{\mathfrak{m}}(V, \mathbf{k}),
$$

defined by $\psi=\mathrm{SC}\left(\varepsilon_{N_{B, A}^{\vee}}\right) \circ-$ and

$$
\phi=\mathrm{id} \otimes-\circ \sum_{n, m \geq 0} \operatorname{Sym}_{n} \otimes \operatorname{Sym}_{m} \otimes \mathrm{id} \circ \mathrm{id}^{\otimes m} \otimes \pi_{V}^{(m)} \circ \mathrm{id}^{\otimes n} \otimes r_{\mathfrak{a}}^{(m)} \circ\left(\pi_{V}^{*}\right)^{(n)},
$$

are well-defined and inverse of each other. It follows that $\mathrm{SC}\left(N_{B, A}^{\vee}\right)$ satisfies the universal property of $N_{+}^{\vee}$, and it is therefore isomorphic to $N_{+}^{\vee}$ in DY $\mathfrak{b}$. Similarly for the structure of Drinfeld-Yetter $\mathfrak{a}^{o p}$-module. 
4.12. Quantum restriction functor. Let $(B, A)$ be a split pair of QUEs. The relative quantum Verma modules $L_{B, A}, N_{B, A}^{\vee}$ allow to define the functor

$$
F_{A, B}: \mathrm{DY}_{B}^{\mathrm{adm}} \rightarrow \mathrm{DY}_{A}^{\mathrm{adm}} \quad F_{A, B}(\mathcal{V})=\operatorname{Hom}_{B}^{B}\left(L_{B, A}, N_{B, A}^{\vee} \otimes \mathcal{V}\right)
$$

Proposition. The functor $F_{A, B}$ is tensor isomorphic to the restriction functor $\operatorname{Res}_{A, B}: \mathrm{DY}_{B}^{\mathrm{adm}} \rightarrow \mathrm{DY}_{A}^{\mathrm{adm}}$.

Proof. For any $\mathcal{V} \in \mathrm{DY}_{B}^{\mathrm{adm}}$, the description of the isomorphism $F_{A, B}(\mathcal{V}) \simeq \mathcal{V}$ as admissible Drinfeld-Yetter $A$-module is identical to that of Proposition 3.14, and relies on the admissibility of $L_{B, A}$ and $N_{B, A}^{\vee}$ and their universal properties in DY ${ }_{A}^{\text {adm }}$.

It is immediate to verify that the tensor structure $J_{A, B}$ on $F_{A, B}$ is trivial, and therefore $F_{A, B}$ is isomorphic to $\operatorname{Res}_{A, B}$ as tensor functors. Namely, for every $v \in$ $F_{A, B}(\mathcal{V}), w \in F_{A, B}(\mathcal{W})$, one has

$$
\begin{aligned}
\left(1_{+} \otimes \mathrm{id} \otimes \mathrm{id}\right) & J_{A, B}(v \otimes w)\left(1_{-}\right) \\
& =\left(1_{+} \otimes \mathrm{id} \otimes \mathrm{id}\right)\left(i_{+}^{\hbar} \otimes \mathrm{id} \otimes \mathrm{id}\right) \circ R_{23}^{-1}(23) \circ v\left(1_{-}\right) \otimes w\left(1_{-}\right) \\
& =\left(1_{+} \otimes \mathrm{id} \otimes \mathrm{id}\right)\left(i_{+}^{\hbar} \otimes \mathrm{id} \otimes \mathrm{id}\right) \circ(23) \circ v\left(1_{-}\right) \otimes w\left(1_{-}\right) \\
& =\left(1_{+} \otimes \mathrm{id}\right) v\left(1_{-}\right) \otimes\left(1_{+} \otimes \mathrm{id}\right) w\left(1_{-}\right),
\end{aligned}
$$

where the second identification follows from the fact that the coaction on $v\left(1_{-}\right)$, $w\left(1_{-}\right)$is trivial.

4.13. Quantisation of $L_{-}$and $N_{+}^{\vee}$. The following is a relative analogue of Proposition 4.5

Theorem. The following holds in the category of Drinfeld-Yetter $\left(U_{\hbar} \mathfrak{b}, U_{\hbar} \mathfrak{a}_{-}\right)-$ modules

(a) $\widetilde{F}_{\mathfrak{b}} \circ \widetilde{F}_{\mathfrak{a}}\left(L_{-}\right) \simeq L_{-}^{\hbar}$ as coalgebras,

(b) $\widetilde{F}_{\mathfrak{b}} \circ \widetilde{F}_{\mathfrak{a}}\left(N_{+}^{\vee}\right) \simeq\left(N_{+}^{\vee}\right)^{\hbar}$ as algebras.

The proof amounts to constructing the intertwiners corresponding to the universal properties of $L_{-}^{\hbar},\left(N_{+}^{\vee}\right)^{\hbar}$. A direct construction along the lines of the proof of Proposition 4.5 is not straightforward, however. We prove this theorem in Section $\S 7$ by describing the modules $L_{-}, N_{+}^{\vee}$, and their quantisation, in the framework of PROPs. These descriptions show that the quantisation of the classical intertwiners of $L_{-}$and $N_{+}^{\vee}$ satisfy the required properties and yield canonical identifications

$$
\widetilde{F}_{\mathfrak{b}} \widetilde{F}_{\mathfrak{a}}\left(L_{-}\right) \simeq L_{-}^{\hbar}, \quad \widetilde{F}_{\mathfrak{b}} \widetilde{F}_{\mathfrak{a}}\left(N_{+}^{\vee}\right) \simeq\left(N_{+}^{\vee}\right)^{\hbar} .
$$

\subsection{The natural transformation $v_{\mathfrak{a}, \mathfrak{b}}$.}

Theorem. Let $\mathfrak{a} \hookrightarrow \mathfrak{b}$ be a split pair of Lie bialgebras, $\mathfrak{g}_{\mathfrak{a}} \hookrightarrow \mathfrak{g}_{\mathfrak{b}}$ the corresponding inclusion of Manin triples, and

$$
\left(\operatorname{Res}_{\mathfrak{a}, \mathfrak{b}}, J_{\mathfrak{a}, \mathfrak{b}}\right): \mathrm{DY}_{\mathfrak{b}}^{\Phi} \rightarrow \mathrm{DY}_{\mathfrak{a}}^{\Phi}
$$


the tensor restriction functor constructed in Section $\S 3$. Then, there exists a natural isomorphism $v_{\mathfrak{a}, \mathfrak{b}}$ such that the following is a commutative diagram

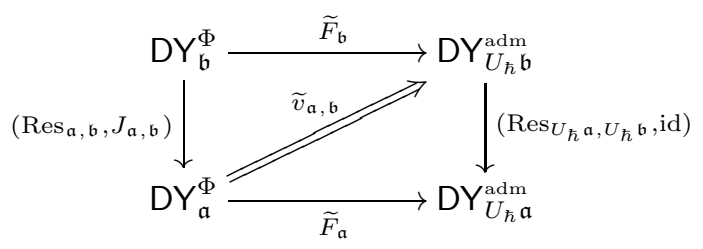

where $\operatorname{Res}_{U_{\hbar} \mathfrak{b}, U_{\hbar} \mathfrak{a}}$ is the functor induced by the split embedding $U_{\hbar} \mathfrak{a} \hookrightarrow U_{\hbar} \mathfrak{b}$.

Proof. By construction of the tensor structure $J_{\mathfrak{a}, \mathfrak{b}}$ and Proposition 4.12 , it is equivalent to prove the commutativity of the above diagram when $\operatorname{Res}_{\mathfrak{a}, \mathfrak{b}}$ and $\operatorname{Res}_{U_{\hbar} \mathfrak{a}, U_{\hbar} \mathfrak{b}}$ are replaced by the tensor restriction functors $F_{\mathfrak{a}, \mathfrak{b}}$ and $F_{\mathfrak{a}, \mathfrak{b}}^{\hbar}:=F_{U_{\hbar} \mathfrak{a}_{-}, U_{\hbar} \mathfrak{b}}$, respectively. Let now $V \in \mathrm{DY}_{\mathfrak{b}}^{\Phi}$, then

$$
\begin{aligned}
F_{\mathfrak{a}, \mathfrak{b}}^{\hbar} \circ \widetilde{F}_{\mathfrak{b}}(V) & =\operatorname{Hom}_{\mathrm{DY}_{U_{\hbar} \mathfrak{b}}}\left(L_{-}^{\hbar},\left(N_{+}^{\vee}\right)^{\hbar} \otimes \widetilde{F}_{\mathfrak{b}}(V)\right) \\
& \cong \operatorname{Hom}_{\mathrm{DY}}\left(\widetilde{F}_{U_{\hbar} \mathfrak{b}} \circ \widetilde{F}_{\mathfrak{a}}\left(L_{-}\right), \widetilde{F}_{\mathfrak{b}} \circ \widetilde{F}_{\mathfrak{a}}\left(N_{+}^{\vee}\right) \otimes \widetilde{F}_{\mathfrak{b}}(V)\right) \\
& \cong \operatorname{Hom}_{\mathrm{DY}_{\mathfrak{b}}^{\Phi}}\left(\widetilde{F}_{\mathfrak{a}}\left(L_{-}\right), \widetilde{F}_{\mathfrak{a}}\left(N_{+}^{\vee}\right) \otimes V\right) \\
& =\widetilde{F}_{\mathfrak{a}}\left(\operatorname{Hom}_{\mathrm{DY}_{\mathfrak{b}}^{\Phi}}\left(L_{-}, N_{+}^{\vee} \otimes V\right)\right) \\
& =\widetilde{F}_{\mathfrak{a}} \circ F_{\mathfrak{a}, \mathfrak{b}}(V),
\end{aligned}
$$

where the first isomorphism follows by Theorem 4.13, the second one from the fact that $\widetilde{F}_{\mathfrak{b}}$ is a tensor equivalence, the subsequent equality from the fact that $L_{-}$is a trivial Drinfeld-Yetter module over $\mathfrak{a}$, and the final one by definition of $F_{\mathfrak{a}, \mathfrak{b}}$. This gives rise to an isomorphism $\widetilde{v}_{\mathfrak{a}, \mathfrak{b}}: F_{\mathfrak{a}, \mathfrak{b}}^{\hbar} \circ \widetilde{F}_{\mathfrak{b}} \cong \widetilde{F}_{\mathfrak{a}} \circ F_{\mathfrak{a}, \mathfrak{b}}$ which is readily seen to preserve the tensor structures.

\section{Quantisation of Split Pairs of Lie Bialgebras}

In this section, we provide a complementary interpretation of the natural isomorphism constructed in the previous section, which relies on a generalisation of the results described in $\S 2.16$.

Specifically, we show that the relative twist on the functor $F_{\mathfrak{a}, \mathfrak{b}}$ gives rise to a Hopf algebra $U_{\hbar} \mathfrak{m}$ in the braided tensor category $\mathrm{DY}_{U_{\hbar} \mathfrak{a}}^{\text {adm }}$. This allows to construct, through the Radford biproduct of $U_{\hbar} \mathfrak{m}$ and $U_{\hbar} \mathfrak{a}$, an alternative quantisation of the split pair $(\mathfrak{b}, \mathfrak{a})$. The natural isomorphism $v_{\mathfrak{a}, \mathfrak{b}}$ provides an isomorphism between this quantisation and $U_{\hbar} \mathfrak{b}$, which restricts to the identity on $U_{\hbar} \mathfrak{a}$.

5.1. Lifting the tensor functor $F_{\mathfrak{a}, \mathfrak{b}}$. The tensor structure $J_{\mathfrak{a}, \mathfrak{b}}$ on the functor $F_{\mathfrak{a}, \mathfrak{b}}$ induces on the algebra End $\left(F_{\mathfrak{a}, \mathfrak{b}}\right)$ a bialgebra structure with coproduct defined for every $\phi \in \operatorname{End}\left(F_{\mathfrak{a}, \mathfrak{b}}\right)$ by the relation

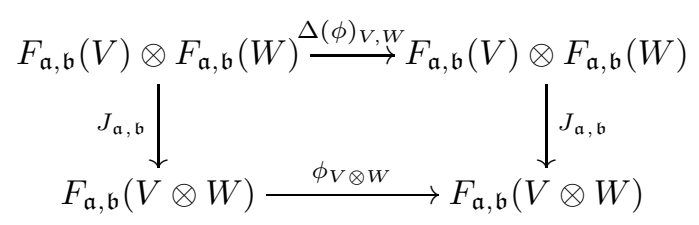


for every $\phi \in \operatorname{End}\left(F_{\mathfrak{a}, \mathfrak{b}}\right)$ and $V, W \in \mathrm{DY}_{\mathfrak{b}}^{\Phi}$, and counit given by evaluation on the trivial module $V=K \in \mathrm{DY}_{\mathfrak{b}}^{\Phi}$. The object $F_{\mathfrak{a}, \mathfrak{b}}\left(L_{-}\right)$naturally embeds in End $\left(F_{\mathfrak{a}, \mathfrak{b}}\right)$ and inherits a (non-topological) Hopf algebra structure. It is essential to observe that these structures are constructed in $D Y_{\mathfrak{a}}^{\Phi}$, and therefore the notion of Hopf algebra is adapted to the associativity and commutativity constraints in $\mathrm{DY}_{\mathfrak{a}}^{\Phi}$. More specifically, we prove the following

\section{Theorem.}

(i) The object $F_{\mathfrak{a}, \mathfrak{b}}\left(L_{-}\right)$is a Hopf algebra in the category $\mathrm{DY} \mathrm{Y}_{\mathfrak{a}}^{\Phi}$.

(ii) The tensor functor $\left(F_{\mathfrak{a}, \mathfrak{b}}, J_{\mathfrak{a}, \mathfrak{b}}\right)$ naturally lifts to a braided tensor functor $\widetilde{F}_{\mathfrak{a}, \mathfrak{b}}$ from $\mathrm{DY}_{\mathfrak{b}}^{\Phi}$ to the category of Drinfeld-Yetter $F_{\mathfrak{a}, \mathfrak{b}}\left(L_{-}\right)$-modules in $\mathrm{DY}_{\mathrm{a}}^{\Phi}$.

(iii) For $\mathfrak{a}=0$, the Hopf algebra $F_{\mathfrak{a}, \mathfrak{b}}\left(L_{-}\right)$is the Etingof-Kazhdan quantisation of $\mathfrak{b}$ and $\widetilde{F}_{\mathfrak{a}, \mathfrak{b}}$ is the braided tensor equivalence 2.23. For $\mathfrak{a}=\mathfrak{b}, \widetilde{F}_{\mathfrak{a}, \mathfrak{b}}$ is the identity functor on $\mathrm{DY}_{\mathfrak{b}}^{\Phi}$.

The proof is carried out in $\S 5.1 .1-\S 5.1 .4$.

5.1.1. Algebra structure on $F_{\mathfrak{a}, \mathfrak{b}}\left(L_{-}\right)$. For every $V \in \mathrm{DY}_{\mathfrak{b}}^{\Phi}$, we define a map $\mu_{V}$ : $F_{\mathfrak{a}, \mathfrak{b}}\left(L_{-}\right) \otimes F_{\mathfrak{a}, \mathfrak{b}}(V) \rightarrow F_{\mathfrak{a}, \mathfrak{b}}(V)$

$$
\mu_{V}(x \otimes v)=\left(i_{+}^{\vee} \otimes \mathrm{id}\right) \Phi_{\mathfrak{b}}{ }^{-1}(\mathrm{id} \otimes v) x .
$$

Let $u \in F_{\mathfrak{a}, \mathfrak{b}}\left(L_{-}\right)$be the element satisfying $u\left(1_{-}\right)=\varepsilon \otimes 1_{-}$.

Proposition. The map $\mu_{V}$ is a morphism in $\mathrm{DY}_{\mathfrak{a}}^{\Phi}$, it is natural in $V$, and it satisfies

$$
\mu_{V}\left(\mathrm{id} \otimes \mu_{V}\right) \Phi_{\mathfrak{a}}=\mu_{V}\left(\mu_{L_{-}} \otimes \mathrm{id}\right) \quad \text { and } \quad \mu_{V} \circ(u \otimes \mathrm{id})=\mathrm{id}_{V} .
$$

Therefore, $\left(F_{\mathfrak{a}, \mathfrak{b}}\left(L_{-}\right), \mu, u\right)$ is an associative algebra object in $\mathrm{DY}_{\mathfrak{a}}^{\Phi}$ acting on the functor $F_{\mathfrak{a}, \mathfrak{b}}$.

Proof. The fact that the map $\mu_{V}$ is a morphism in $\mathrm{DY} \mathrm{q}_{\mathfrak{a}}^{\Phi}$, its naturality in $V$, and its compatibility with $u \in F_{\mathfrak{a}, \mathfrak{b}}\left(L_{-}\right)$are straightforward.

Let now $x, y \in F_{\mathfrak{a}, \mathfrak{b}}\left(L_{-}\right), v \in F_{\mathfrak{a}, \mathfrak{b}}(V)$ and $\Phi_{\mathfrak{a}}=\sum P_{\mathfrak{K}} \otimes Q_{\mathfrak{K}} \otimes R_{\mathfrak{K}}$. Then

$$
\begin{aligned}
\mu_{V} & \left(\mathrm{id} \otimes \mu_{V}\right) \Phi_{\mathfrak{a}}(x \otimes y \otimes v) \\
& =\left(i_{+}^{\vee} \otimes \mathrm{id}\right) \Phi_{\mathfrak{b}}{ }^{-1}\left(\mathrm{id} \otimes i_{+}^{\vee} \otimes \mathrm{id}\right)\left(\mathrm{id} \otimes \Phi_{\mathfrak{b}}{ }^{-1}\right)\left(\mathrm{id} \otimes \mathrm{id} \otimes R_{\mathrm{K}} \cdot v\right)\left(\mathrm{id} \otimes Q_{\mathrm{K}} \cdot y\right)\left(P_{\mathrm{k}} \cdot x\right) \\
& =\left(i_{+}^{\vee} \otimes \mathrm{id}\right)\left(\mathrm{id} \otimes i_{+}^{\vee} \otimes \mathrm{id}\right) \Phi_{\mathfrak{b}}{ }_{1,23,4} \Phi_{\mathfrak{b}}{ }_{234} \Phi_{\mathfrak{a}}{ }_{123}(\mathrm{id} \otimes \mathrm{id} \otimes v)(\mathrm{id} \otimes y) x \\
& =\left(i_{+}^{\vee} \otimes \mathrm{id}\right)\left(\mathrm{id} \otimes i_{+}^{\vee} \otimes \mathrm{id}\right) \Phi_{\mathfrak{b}_{123}} \Phi_{\mathfrak{a}_{123}}{ }_{123} \Phi_{\mathfrak{b}}{ }_{12,3,4}^{-1} \Phi_{\mathfrak{b}}{ }_{1,2,34}^{-1}(\mathrm{id} \otimes \mathrm{id} \otimes v)(\mathrm{id} \otimes y) x \\
& =\left(i_{+}^{\vee} \otimes \mathrm{id}\right)\left(i_{+}^{\vee} \otimes \mathrm{id} \otimes \mathrm{id}\right) \Phi_{\mathfrak{b}}{ }_{12,3,4}{ }_{\mathfrak{b}_{1,2,34}}^{-1}(\mathrm{id} \otimes \mathrm{id} \otimes v)(\mathrm{id} \otimes y) x \\
& =\left(i_{+}^{\vee} \otimes \mathrm{id}\right) \Phi_{\mathfrak{b}}{ }^{-1}(\mathrm{id} \otimes \mathrm{id} \otimes v)\left(i_{+}^{\vee} \otimes \mathrm{id}\right) \Phi_{\mathfrak{b}}{ }^{-1}(\mathrm{id} \otimes y) x \\
& =\mu_{V}\left(\mu_{L_{-}} \otimes \mathrm{id}\right)(x \otimes y \otimes v)
\end{aligned}
$$

by applications of the pentagon axiom and the associativity of $i_{+}^{\vee}$. 
5.1.2. Coalgebra structure on $F_{\mathfrak{a}, \mathfrak{b}}\left(L_{-}\right)$. The coproduct $i_{-}$on $L_{-}$induces a coproduct on $F_{\mathfrak{a}, \mathfrak{b}}\left(L_{-}\right)$by the formula

$$
\Delta_{L}: F_{\mathfrak{a}, \mathfrak{b}}\left(L_{-}\right) \rightarrow F_{\mathfrak{a}, \mathfrak{b}}\left(L_{-}\right) \otimes F_{\mathfrak{a}, \mathfrak{b}}\left(L_{-}\right) \quad \Delta_{L}=J_{\mathfrak{a}, \mathfrak{b}}^{-1} \circ F_{\mathfrak{a}, \mathfrak{b}}\left(i_{-}\right) .
$$

Proposition. The map $\Delta_{L}$ is a morphism in $\mathrm{DY}_{\mathfrak{a}}^{\Phi}$ and it satisfies

$$
\Phi_{\mathfrak{a}}\left(\Delta_{L} \otimes \mathrm{id}\right) \Delta_{L}=\left(\mathrm{id} \otimes \Delta_{L}\right) \Delta_{L} .
$$

Proof. The maps $J_{\mathfrak{a}, \mathfrak{b}}$ and $F_{\mathfrak{a}, \mathfrak{b}}\left(i_{-}\right)$are both morphisms $\mathrm{DY}_{\mathfrak{a}}^{\Phi}$, therefore $\Delta_{L}$ is. Moreover,

$$
\begin{aligned}
\Phi_{\mathfrak{a}}\left(\Delta_{L} \otimes \mathrm{id}\right) \Delta_{L} & =\Phi_{\mathfrak{a}} J_{F_{\mathfrak{a}, \mathfrak{b}}, 1,2}^{-1}\left(F_{\mathfrak{a}, \mathfrak{b}}\left(i_{-}\right) \otimes \mathrm{id}\right) J_{\mathfrak{a}, \mathfrak{b}}^{-1} F_{\mathfrak{a}, \mathfrak{b}}\left(i_{-}\right) \\
& =\Phi_{\mathfrak{a}} J_{F_{\mathfrak{a}, \mathfrak{b}}, 1,2}^{-1} J_{F_{\mathfrak{a}, \mathfrak{b}}, 12,3}^{-1} F_{\mathfrak{a}, \mathfrak{b}}\left(i_{-} \otimes \mathrm{id}\right) F_{\mathfrak{a}, \mathfrak{b}}\left(i_{-}\right) \\
& =J_{F_{\mathfrak{a}, \mathfrak{b}}, 2,3}^{-1} J_{F_{\mathfrak{a}, \mathfrak{b}}, 1,23}^{-1} F_{\mathfrak{a}, \mathfrak{b}}\left(\Phi_{\mathfrak{b}}\right) F_{\mathfrak{a}, \mathfrak{b}}\left(i_{-} \otimes \mathrm{id}\right) F_{\mathfrak{a}, \mathfrak{b}}\left(i_{-}\right) \\
& =J_{F_{\mathfrak{a}, \mathfrak{b}}, 2,3}^{-1}\left(\mathrm{id} \otimes F_{\mathfrak{a}, \mathfrak{b}}\left(i_{-}\right)\right) J_{\mathfrak{a}, \mathfrak{b}}^{-1} F_{\mathfrak{a}, \mathfrak{b}}\left(i_{-}\right) \\
& =\left(\operatorname{id} \otimes \Delta_{L}\right) \Delta_{L} .
\end{aligned}
$$

For simplicity, from now on, we omit the action of the associator $\Phi_{\mathfrak{a}}$ since we proved that it is natural with respect to $\mu, \Delta_{L}$ and $J_{\mathfrak{a}, \mathfrak{b}}$.

5.1.3. Relation with End $\left(F_{\mathfrak{a}, \mathfrak{b}}\right)$. It follows from Proposition 5.1.1 that the collection of morphisms $\mu_{V}, V \in \mathrm{DY}_{\mathfrak{b}}^{\Phi}$, defines a morphism of algebras from $F_{\mathfrak{a}, \mathfrak{b}}\left(L_{-}\right)$to End $\left(F_{\mathfrak{a}, \mathfrak{b}}\right)$, whose injectivity follows immediately from the action of $F_{\mathfrak{a}, \mathfrak{b}}\left(L_{-}\right)$on itself. We now show that this embedding is in fact a morphism of coalgebras. To this extent, we denote by $\Delta$ the coproduct on $\operatorname{End}\left(F_{\mathfrak{a}, \mathfrak{b}}\right)$, by $\varphi^{(1)}$ the embedding defined in $\S 5.1 .1$, and by $\varphi^{(2)}$ the embedding from $F_{\mathfrak{a}, \mathfrak{b}}\left(L_{-}\right)^{\otimes 2} \operatorname{into}$ End $\left(F_{\mathfrak{a}, \mathfrak{b}}^{2}\right)$ defined for every $V, W \in \mathrm{DY}_{\mathfrak{a}}^{\Phi}$ by

$$
\varphi_{V, W}^{(2)}=\left(\mu_{V} \otimes \mu_{W}\right) \circ \beta_{\mathfrak{a}, 23}
$$

as a map from $F_{\mathfrak{a}, \mathfrak{b}}\left(L_{-}\right) \otimes F_{\mathfrak{a}, \mathfrak{b}}\left(L_{-}\right) \otimes F_{\mathfrak{a}, \mathfrak{b}}(V) \otimes \mathcal{F} F_{\mathfrak{b}}(W)$ to $F_{\mathfrak{a}, \mathfrak{b}}(V) \otimes F_{\mathfrak{a}, \mathfrak{b}}(W)$.

Proposition. One has

$$
\Delta \circ \varphi^{(1)}=\varphi^{(2)} \circ \Delta_{L}
$$

In particular, the counit of End $\left(F_{\mathfrak{a}, \mathfrak{b}}\right)$, i.e., the evaluation on the identity object $V=K \in \mathrm{DY}_{\mathfrak{b}}^{\Phi}$, restricts to a counit $\varepsilon=\mu_{K}$ on $F_{\mathfrak{a}, \mathfrak{b}}\left(L_{-}\right)$and the object $\left(F_{\mathfrak{a}, \mathfrak{b}}\left(L_{-}\right), \mu_{L}, u, \Delta_{L}, \varepsilon\right)$ is a bialgebra object in $\mathrm{DY}_{\mathfrak{a}}^{\Phi}$.

Proof. From the definition of $\Delta$, it follows that (5.1) is equivalent to

$$
\mu_{V \otimes W}\left(\mathrm{id} \otimes J_{\mathfrak{a}, \mathfrak{b}}\right)=J_{\mathfrak{a}, \mathfrak{b}}\left(\mu_{V} \otimes \mu_{W}\right) \beta_{\mathfrak{a}, 23}\left(\Delta_{L} \otimes \mathrm{id} \otimes \mathrm{id}\right)
$$

as morphisms from $F_{\mathfrak{a}, \mathfrak{b}}\left(L_{-}\right) \otimes F_{\mathfrak{a}, \mathfrak{b}}(V) \otimes F_{\mathfrak{a}, \mathfrak{b}}(W)$ to $F_{\mathfrak{a}, \mathfrak{b}}(V \otimes W)$, where $V, W \in$ $\mathrm{DY}_{\mathfrak{b}}^{\Phi}$. In $F_{\mathfrak{a}, \mathfrak{b}}(V \otimes W)$, one has to show

$\left(i_{+}^{\vee} \otimes \mathrm{id} \otimes \mathrm{id}\right)\left(\mathrm{id} \otimes i_{+}^{\vee} \otimes \mathrm{id} \otimes \mathrm{id}\right) \beta_{34}(\mathrm{id} \otimes v \otimes w)\left(\mathrm{id} \otimes i_{-}\right) x$

$$
=\left(i_{+}^{\vee} \otimes \mathrm{id} \otimes \mathrm{id}\right) \beta_{23}\left(i_{+}^{\vee} \otimes \mathrm{id} \otimes i_{+}^{\vee} \otimes \mathrm{id}\right)\left(\mathrm{id} \otimes b_{i} \cdot v \otimes \mathrm{id} \otimes w\right)\left(x_{1} \otimes a_{i} . x_{2}\right) i_{-},
$$

where $v \in F_{\mathfrak{a}, \mathfrak{b}}(V), w \in F_{\mathfrak{a}, \mathfrak{b}}(W), x \in F_{\mathfrak{a}, \mathfrak{b}}\left(L_{-}\right)$, and, in sumless Sweedler notation, $\Delta_{L}(x)=x_{1} \otimes x_{2}, \exp \left(-\hbar \Omega_{\mathfrak{a}} / 2\right)=a_{i} \otimes b_{i}$. 
It is more convenient, in this case, to give a pictorial proof, relying on the graphical calculus for Lie bialgebras (e.g. [17, Lecture 19]). We read the diagrams from top to bottom and from left to right.

We have to show that the following diagrams are equivalent:
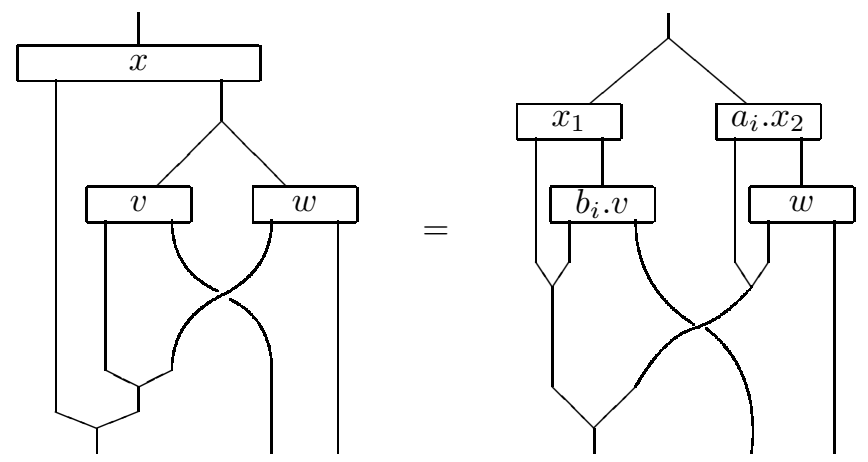

The coproduct $\Delta_{L}$ is defined by the equation
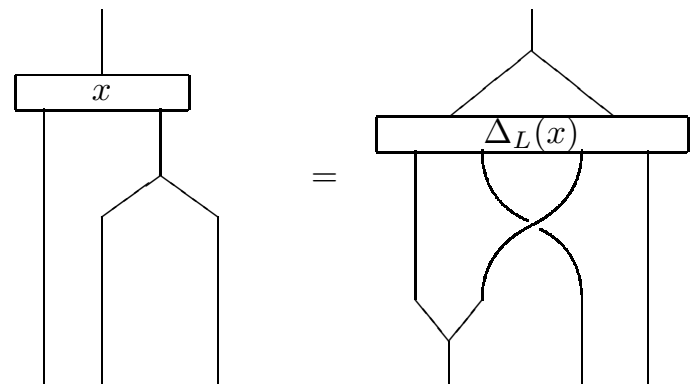

Since the action of $\mathfrak{g}_{\mathfrak{a}}$ on the objects $F_{\mathfrak{a}, \mathfrak{b}}(V)$ is given by right action on $N_{+}^{\vee}$, we represent the braiding $\beta_{\mathfrak{a}}^{-1}$ as

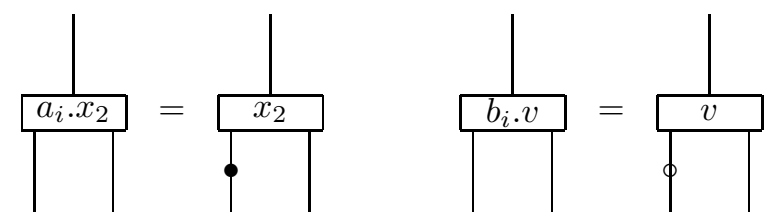

We are allowed to move the black and the white bullets along the lines, since they commute with the left action of $\mathfrak{g}_{\mathfrak{b}}$. The RHS corresponds to
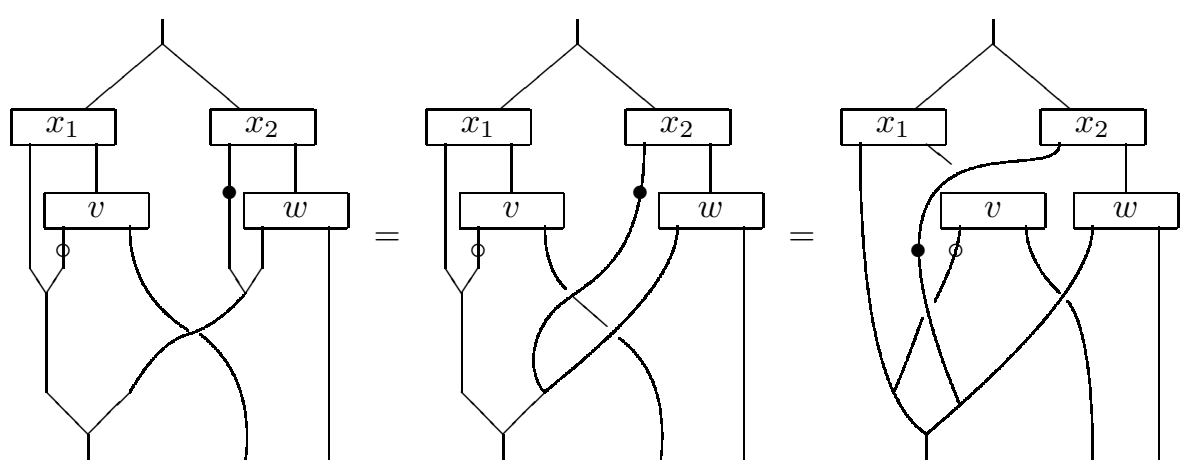
We now use the fact that the map $i_{+}^{\vee}$ satisfies $i_{+}^{\vee} \circ \beta \circ\left(\beta_{\mathfrak{a}}^{-1}\right)^{\rho}=i_{+}^{\vee}$, i.e.,

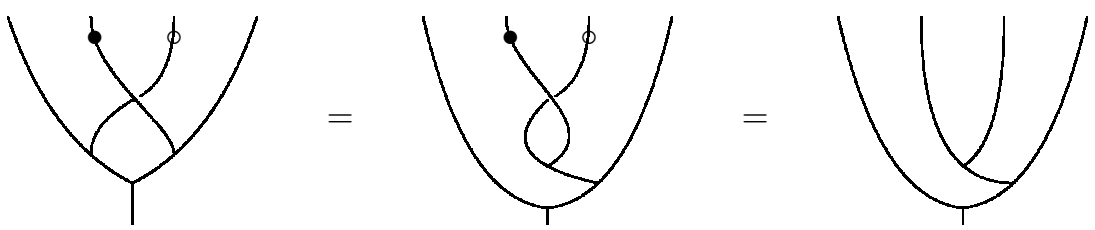

Finally we get

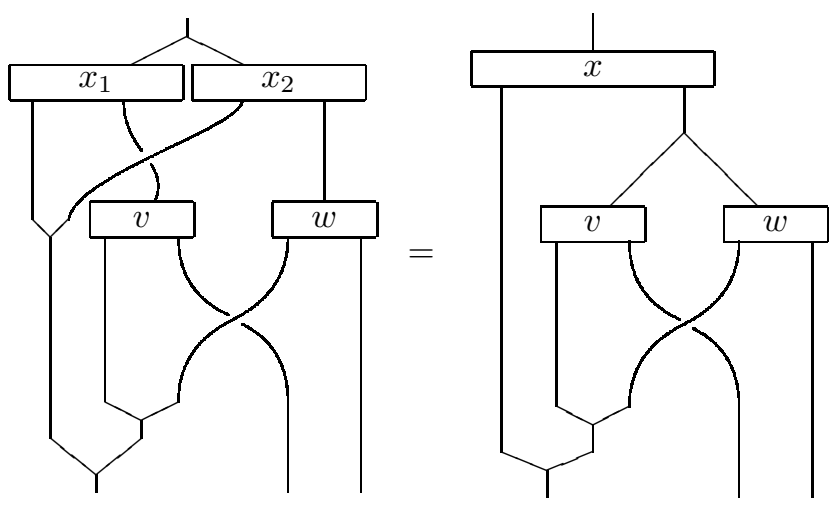

It follows immediately from (5.1) that the restriction of the counit $\varepsilon$ of End $\left(F_{\mathfrak{a}, \mathfrak{b}}\right)$ to $F_{\mathfrak{a}, \mathfrak{b}}\left(L_{-}\right)$satisfies

$$
(\varepsilon \otimes \mathrm{id}) \circ \Delta_{L}=\mathrm{id}=(\mathrm{id} \otimes \varepsilon) \circ \Delta_{L} .
$$

The compatiblity of product and coproduct on $F_{\mathfrak{a}, \mathfrak{b}}\left(L_{-}\right)$follows from that in End $\left(F_{\mathfrak{a}, \mathfrak{b}}\right)$, and the tuple $\left(F_{\mathfrak{a}, \mathfrak{b}}\left(L_{-}\right), \mu, u, \Delta_{L}, \varepsilon\right)$ gives a bialgebra object in $\mathrm{DY}_{\mathfrak{a}}^{\Phi}$. Moreover, since $F_{\mathfrak{a}, \mathfrak{b}}\left(L_{-}\right)$reduces to $U \mathfrak{m}_{-}$modulo $\hbar$, there exists a unique antipode defining $F_{\mathfrak{a}, \mathfrak{b}}\left(L_{-}\right)$a Hopf algebra object in $\mathrm{DY}_{\mathfrak{a}}^{\Phi}$. This complete the proof of part (i) in 5.1 .

5.1.4. Twisted $R$-matrix and coactions. The tensor functor $\left(F_{\mathfrak{a}, \mathfrak{b}}, J_{\mathfrak{a}, \mathfrak{b}}\right)$ induces a natural braiding on the subcategory generated in $\mathrm{DY}_{\mathfrak{a}}^{\Phi}$ by the objects $F_{\mathfrak{a}, \mathfrak{b}}(V)$, for any $V \in \mathrm{DY}_{\mathfrak{b}}^{\Phi}$. The braiding is given by the usual formula

$$
\beta_{J_{\mathfrak{a}, \mathfrak{b}}}=J_{F_{\mathfrak{a}, \mathfrak{b}}, 21}^{-1} \circ F_{\mathfrak{a}, \mathfrak{b}}(\beta) \circ J_{\mathfrak{a}, \mathfrak{b}},
$$

where $\beta$ is the usual braiding in $\mathrm{DY}_{\mathfrak{b}}^{\Phi}$. Clearly, $\beta_{J_{\mathfrak{a}, \mathfrak{b}}}$ is a morphism in $\mathrm{DY} \mathbf{Y}_{\mathfrak{a}}^{\Phi}$.

For every $V \in \mathrm{DY}_{\mathfrak{b}}^{\Phi}$, the object $F_{\mathfrak{a}, \mathfrak{b}}(V)$ is endowed with a trivial comodule structure over $F_{\mathfrak{a}, \mathfrak{b}}\left(L_{-}\right)$by the map

$$
\eta_{V}: F_{\mathfrak{a}, \mathfrak{b}}(V) \rightarrow F_{\mathfrak{a}, \mathfrak{b}}\left(L_{-}\right) \otimes F_{\mathfrak{a}, \mathfrak{b}}(V) \quad \eta_{V}(v)=u \otimes v .
$$

The maps $\eta$ are morphisms in $D Y_{\mathfrak{a}}^{\Phi}$ and they satisfy

$$
\Phi_{\mathfrak{a}}(\eta \otimes \mathrm{id}) \eta_{V}=\left(\mathrm{id} \otimes \eta_{V}\right) \eta_{V}
$$

where $\eta:=\eta_{L_{-}}$and $\eta(u)=u \otimes u=\Delta_{L}(u)$. Set

$$
\mu_{V}^{*}: F_{\mathfrak{a}, \mathfrak{b}}(V) \rightarrow F_{\mathfrak{a}, \mathfrak{b}}\left(L_{-}\right) \otimes F_{\mathfrak{a}, \mathfrak{b}}(V), \quad \mu_{V}^{*}=\mathcal{R}_{V} \circ \eta_{V}
$$

where $\mathcal{R}:=\sigma \cdot \beta_{J_{\mathfrak{a}, \mathfrak{b}}}$ is the relative $R$-matrix. 
Proposition. The map $\mu_{V}^{*}$ defines on $F_{\mathfrak{a}, \mathfrak{b}}(V)$ a structure of comodule over $F_{\mathfrak{a}, \mathfrak{b}}\left(L_{-}\right)$. This is compatible with the action of $F_{\mathfrak{a}, \mathfrak{b}}\left(L_{-}\right)$and defines on $F_{\mathfrak{a}, \mathfrak{b}}(V)$ a DrinfeldYetter structure over $F_{\mathfrak{a}, \mathfrak{b}}\left(L_{-}\right)$.

Proof. For any $V \in \mathrm{DY}_{\mathfrak{b}}^{\Phi}$

$$
\left(\mathrm{id} \otimes \eta_{V}\right) \mathcal{R} \eta_{V}=\mathcal{R}_{23}\left(\mathrm{id} \otimes \eta_{V}\right) \eta_{V}, \quad\left(\eta_{V} \otimes \mathrm{id}\right) \mathcal{R} \eta_{V}=\mathcal{R}_{13}\left(\eta_{V} \otimes \mathrm{id}\right) \eta_{V}
$$

Therefore, by the hexagon relations of $\mathcal{R}$ it follows

$$
\begin{aligned}
\left(\mathrm{id} \otimes \mu_{V}^{*}\right) \mu_{V}^{*} & =\mathcal{R}_{13}\left(\mathrm{id} \otimes \eta_{V}\right) \mathcal{R} \eta_{V} \\
& =\mathcal{R}_{13} \mathcal{R}_{23}\left(\mathrm{id} \otimes \eta_{V}\right) \eta_{V} \\
& =\left(\Delta_{L}{ }^{21} \otimes \mathrm{id}\right) \mu_{V}^{*} .
\end{aligned}
$$

The compatibility with the action is similarly proved, relying on the fact that the relative $R$-matrix defines a quasi-triangular structure on End $\left(F_{\mathfrak{a}, \mathfrak{b}}\right)$.

Let now denote by $\mathrm{DY}_{L, \mathfrak{a}}^{\Phi}$ the braided category of Drinfeld-Yetter $F_{\mathfrak{a}, \mathfrak{b}}\left(L_{-}\right)$modules in $D Y_{\mathfrak{a}}^{\Phi}$ with braiding $\beta_{J_{\mathfrak{a}, \mathfrak{b}}}$. It follows from the last Proposition that the tensor functor $F_{\mathfrak{a}, \mathfrak{b}}$ factors through $\mathrm{DY}_{L, \mathfrak{a}}^{\Phi}$, i.e.,

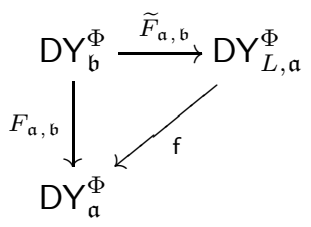

where $f$ denotes the forgetful functor. This completes the proof of part $(i i)$ and (iii) of Theorem 5.1.

5.2. Quantisation of a split pair. The application of Etingof-Kazhdan functor $\widetilde{F}_{\mathfrak{a}}: \mathrm{DY}_{\mathfrak{a}}^{\Phi} \rightarrow \mathrm{DY}_{U_{\hbar} \mathfrak{a}}$ allows to further transform $F_{\mathfrak{a}, \mathfrak{b}}\left(L_{-}\right)$into a Hopf algebra object

$$
U_{\hbar} \mathfrak{m}=\left(\widetilde{F}_{\mathfrak{a}} \circ F_{\mathfrak{a}, \mathfrak{b}}\left(L_{-}\right), \widetilde{F}_{\mathfrak{a}}(\mu), \widetilde{F}_{\mathfrak{a}}(\delta)\right)
$$

in the category DY $\mathrm{U}_{U_{\hbar} \mathfrak{a}}^{\mathrm{adm}}$. The action and coaction of $F_{\mathfrak{a}, \mathfrak{b}}\left(L_{-}\right)$on $F_{\mathfrak{a}, \mathfrak{b}}(V)$ provide through $\widetilde{F}_{\mathfrak{a}}$ an action and an admissible coaction of $U_{\hbar} \mathfrak{m}$ on $\widetilde{F}_{\mathfrak{a}} \circ F_{\mathfrak{a}, \mathfrak{b}}(V)$ in the category $\mathrm{DY}_{U_{\hbar} \mathfrak{a}}$. Namely, we have a diagram of functors

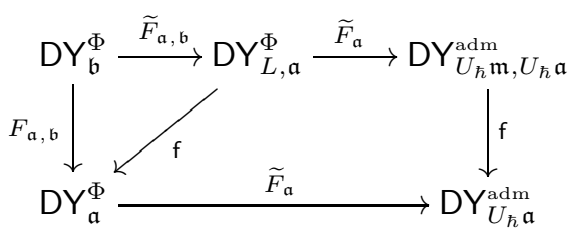

where $\mathrm{DY}_{U_{\hbar} \mathfrak{m}, U_{\hbar} \mathfrak{a}}$ denotes the category of Drinfeld-Yetter modules over $U_{\hbar} \mathfrak{m}$ in $\mathrm{DY}_{U_{\hbar} \mathrm{a}}$. 
5.2.1. Since $U_{\hbar} \mathfrak{m}$ is a Hopf algebra in $\mathrm{DY}_{U_{\hbar} \mathfrak{a}}$, it follows from $\S 4.6-\S 4.8$ that we can construct a Hopf algebra $U_{\hbar}^{\text {rel }} \mathfrak{b}=U_{\hbar} \mathfrak{m} \star U_{\hbar} \mathfrak{a}$, and include the equivalence $\mathrm{DY}_{U_{\hbar} \mathfrak{m}, U_{\hbar} \mathfrak{a}}^{\mathrm{adm}} \simeq \mathrm{DY}_{U_{\hbar}^{\text {rel }} \mathfrak{b}}^{\mathrm{ardm}}$ in the diagram (5.2),

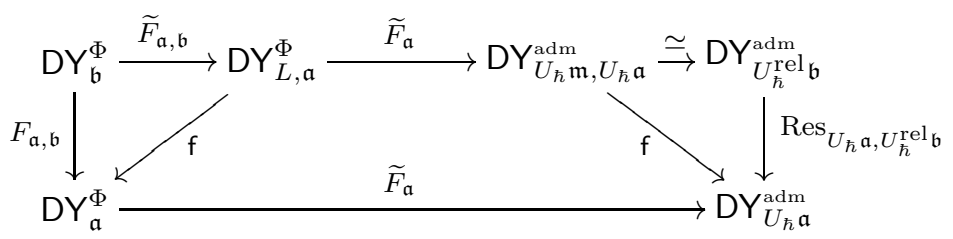

The equivalence $\widetilde{F_{\mathfrak{a}} F_{\mathfrak{a}, \mathfrak{b}}}$, obtained by composition along the top row of (5.3), is a Tannakian lift of the fiber functor $F_{\mathfrak{a}} F_{\mathfrak{a}, \mathfrak{b}}$ with tensor structure given by $J_{\mathfrak{a}}$ and $J_{\mathfrak{a}, \mathfrak{b}}$. The isomorphism $\widetilde{v}_{\mathfrak{a}, \mathfrak{b}}$ constructed in Section $\S 4$ descends to an isomorphism of tensor functors between $F_{\mathfrak{b}}$ and $F_{\mathfrak{a}} \circ F_{\mathfrak{a}, \mathfrak{b}}$, and leads to the following

\section{Theorem.}

(i) The pair $\left(U_{\hbar}^{\text {rel }} \mathfrak{b}, U_{\hbar} \mathfrak{a}\right)$ is a split pair of Hopf algebras quantising the split pair $(\mathfrak{b}, \mathfrak{a})$.

(ii) There is an isomorphism of split pairs of Hopf algebras

$$
v_{\mathfrak{a}, \mathfrak{b}}:\left(U_{\hbar}^{\mathrm{rel}} \mathfrak{b}, U_{\hbar} \mathfrak{a}\right) \simeq\left(U_{\hbar} \mathfrak{b}, U_{\hbar} \mathfrak{a}\right) .
$$

Proof. ( $i$ ) follows by construction. It is in fact immediate to show that the 1 -jet of the composition of the twists $J_{\mathfrak{a}, \mathfrak{b}}$ and $J_{\mathfrak{a}}$ gives back the $r$-matrix of $\mathfrak{b}$.

In order to prove ( $i i)$, we observe that, by functoriality of the quantisation, $\left(U_{\hbar} \mathfrak{b}, U_{\hbar} \mathfrak{a}\right)$ is a split pair and, by Radford theorem, there is an isomorphism of Hopf algebras $U_{\hbar} \mathfrak{b} \simeq L_{-}^{\hbar} \star U_{\hbar} \mathfrak{a}$, where $L_{-}^{\hbar}$ is the Verma module constructed in $\S 4.10$. Through $\widetilde{v}_{\mathfrak{a}, \mathfrak{b}}$ we obtain an identification

$$
\widetilde{F}_{\mathfrak{a}} F_{\mathfrak{a}, \mathfrak{b}}\left(L_{-}\right) \simeq \widetilde{F}_{\mathfrak{b}}\left(L_{-}\right) \simeq L_{-}^{\hbar}
$$

as Hopf algebra objects in DY $U_{U_{\hbar} \mathfrak{a}}$. More specifically, it is clear that (5.4) preserves the structures of coalgebra and Drinfeld-Yetter $U_{\hbar} \mathfrak{a}$-module. The algebra structure on $L_{-}^{\hbar} \simeq \widetilde{F}_{\mathfrak{b}}\left(L_{-}\right)$is induced by its inclusion in $U_{\hbar} \mathfrak{b} \simeq \widetilde{F}_{\mathfrak{b}}\left(M_{-}\right)$, which is a subalgebra in End $\left(F_{\mathfrak{b}}\right)$. Similarly, $\widetilde{F}_{\mathfrak{a}} F_{\mathfrak{a}, \mathfrak{b}}\left(L_{-}\right)$is a subalgebra in End $\left(F_{\mathfrak{a}} F_{\mathfrak{a}, \mathfrak{b}}\right)$, which is isomorphic to End $\left(F_{\mathfrak{b}}\right)$ through $v_{\mathfrak{a}, \mathfrak{b}}$. Therefore, we obtain an isomorphism of Hopf algebras

$$
U_{\hbar} \mathfrak{b} \simeq L_{-}^{\hbar} \star U_{\hbar} \mathfrak{a} \simeq \widetilde{F}_{\mathfrak{a}} F_{\mathfrak{a}, \mathfrak{b}}\left(L_{-}\right) \star U_{\hbar} \mathfrak{a}=U_{\hbar}^{\mathrm{rel}} \mathfrak{b},
$$

which restricts to the identity on $U_{\hbar} \mathfrak{a}$.

\section{UNIVERSAL CONSTRUCTIONS}

We review in this section the PROPic quantisation of a Lie bialgebra $\mathfrak{b}$ following [15]. We then extend that description to the Tannakian functor $\widetilde{F}_{\mathfrak{b}}: \mathrm{DY}_{\mathfrak{b}}^{\Phi} \rightarrow \mathrm{DY}_{U_{\hbar} \mathfrak{b}}^{\mathrm{adm}}$, and use it to give an alternative proof that $\widetilde{F}_{\mathfrak{b}}$ is an equivalence.

6.1. PROPs [23, 21]. A PROP is a $\mathbf{k}$-linear, strict, symmetric monoidal category $\mathcal{C}$ whose objects are the non-negative integers, and such that $[n] \otimes[m]=[n+m]$. In particular $[0]$ is the unit object, and $[1]^{\otimes n}=[n]$. A morphism between two PROPs $\mathcal{C}, \mathcal{D}$ is a symmetric tensor functor $\mathcal{G}: \mathcal{C} \rightarrow \mathcal{D}$, which is the identity on objects, i.e., $\mathcal{G}\left([n]_{\mathcal{C}}\right)=[n]_{\mathcal{D}}, n \geqslant 0$, and has a trivial tensor structure. 
6.2. Associative algebras. Let Alg be the PROP whose morphisms are generated by two elements $\iota:[0] \rightarrow[1]$ (the unit) and $m:[2] \rightarrow[1]$ (the multiplication) satisfying the relations

$$
\begin{gathered}
m \circ\left(m \otimes \operatorname{id}_{[1]}\right)=m \circ\left(\operatorname{id}_{[1]} \otimes m\right), \\
m \circ\left(\iota \otimes \operatorname{id}_{[1]}\right)=\operatorname{id}_{[1]}=m \circ\left(\operatorname{id}_{[1]} \otimes \iota\right) .
\end{gathered}
$$

A (unital, associative) $k$-algebra is the same as a symmetric tensor functor Alg $\rightarrow$ Vect $t_{k}$. More precisely, there is functor ev from the category Fun ${ }^{\otimes}\left(\mathrm{Alg}\right.$, Vect $_{k}$ ) of symmetric tensor functors from Alg to $\operatorname{Vect}_{k}$, to the category $\mathrm{Alg}\left(\operatorname{Vect}_{\mathrm{k}}\right)$ of $\mathrm{k}$ algebras. The functor ev sends $(\mathcal{G}, J)$ to $\left(\mathcal{G}([1]), \mathcal{G}(m) \circ J_{[1],[1]}, \mathcal{G}(\iota)\right)$, and is easily seen to be an equivalence of categories.

The equivalence ev may be restricted to an isomorphism as follows. Choose a family $\mathbf{b}=\left\{b_{n}\right\}_{n \geq 2}$, where $b_{n}$ is a complete bracketing on $x_{1} \cdots x_{n}$, and restrict ev to the subcategory $\operatorname{Fun}_{\mathbf{b}}^{\otimes}\left(\mathrm{Alg}\right.$, Vect $\left._{\mathrm{k}}\right)$ of symmetric tensor functors $(\mathcal{G}, J)$ such that

$$
\mathcal{G}([n])=\mathcal{G}([1])_{b_{n}}^{\otimes n}
$$

and the following diagram is commutative

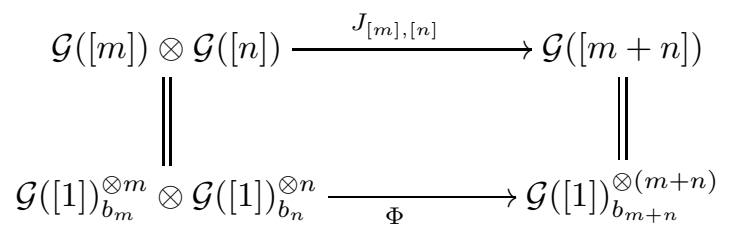

where $\Phi$ is the corresponding associativity constraint in Vect. Then, ev restricts to an isomorphism of categories

$$
\text { ev : } \operatorname{Fun}_{\mathbf{b}}^{\otimes}\left(\mathrm{Alg}, \operatorname{Vect}_{\mathrm{k}}\right) \rightarrow \operatorname{Alg}\left(\text { Vect }_{\mathrm{k}}\right) .
$$

Moreover, it is clear that, for any choices $\mathbf{b}, \mathbf{b}^{\prime}$, there is a canonical isomorphism Fun $_{\mathbf{b}}^{\otimes}\left(\right.$ Alg, Vect $\left.{ }_{k}\right) \rightarrow$ Fun $_{\mathbf{b}^{\prime}}^{\otimes}\left(\right.$ Alg, Vect $\left._{k}\right)$.

6.3. Modules over a PROP. The discussion in 6.2 may be extended to an arbitrary PROP P as follows. Fix henceforth a choice $\mathbf{b}=\left\{b_{n}\right\}_{n \geq 2}$ of bracketings.

Definition. A module over $\mathrm{P}$ in a symmetric monoidal category $\mathcal{N}$ is a symmetric tensor functor $\mathrm{P} \rightarrow \mathcal{N}$ such that (6.1) and (6.2) above hold, where $\Phi$ is the associativity constraint in $\mathcal{N}$. A morphism of modules over $\mathrm{P}$ is a natural transformation of functors. The category of $\mathrm{P}$-modules is denoted by $\operatorname{Fun}_{\mathbf{b}}^{\otimes}(\mathrm{P}, \mathcal{N})$.

In particular, by definition, a Alg-module in Vect $\mathrm{k}_{\mathrm{k}}$ is a $\mathrm{k}$-algebra. The notion of $\mathrm{P}$-module can be rephrased in term of morphisms of PROPs (cf. [11, §2.4]). Namely, for any object $X \in \mathcal{N}$, we can consider the PROP $\mathrm{P}_{\mathbf{b}, X}$ with morphisms

$$
\mathrm{P}_{\mathbf{b}, X}([n],[m])=\operatorname{Hom}_{\mathcal{N}}\left(X_{b_{n}}^{\otimes n}, X_{b_{m}}^{\otimes m}\right)
$$

Then, $X$ is a $\mathrm{P}$-module in $\mathcal{N}$ if and only if there is a morphism of PROPs $\mathrm{P} \rightarrow \mathrm{P}_{\mathbf{b}, X}$.

6.4. Universal Constructions. Let LA be the PROP generated by a morphism $\mu:[2] \rightarrow[1]$ (the bracket), subject to the relations

$$
\mu \circ\left(\operatorname{id}_{[2]}+(12)\right)=0 \quad \text { and } \quad \mu \circ\left(\mu \otimes \operatorname{id}_{[1]}\right) \circ\left(\operatorname{id}_{[3]}+(123)+(312)\right)=0
$$

as morphisms [2] $\rightarrow$ [1] and [3] $\rightarrow$ [1] respectively. Thus, a LA-module in Vect k $_{\mathrm{k}}$ is k-Lie algebra. 
The fact that any associative algebra $A$ is naturally a Lie algebra may be described in terms of PROPs as follows. Let $\left(\mathcal{G}_{A}, J_{A}\right) \in \mathrm{Fun}_{\mathbf{b}}^{\otimes}\left(\mathrm{Alg}\right.$, Vect , $\left._{\mathrm{k}}\right)$ be such that $\mathcal{G}_{A}[1]=A$. The corresponding Lie algebra arises from the composition

$$
\mathrm{LA} \stackrel{L}{\longrightarrow} \mathrm{Alg} \stackrel{\left(\mathcal{G}_{A}, J_{A}\right)}{\longrightarrow} \operatorname{Vect}_{\mathrm{k}}
$$

where $L: \mathrm{LA} \rightarrow \mathrm{Alg}$ is the strict symmetric tensor functor mapping [1] $\mathrm{LA}$ to $[1]_{\mathrm{Alg}}$, and $\mu$ to $m-m \circ(21)$. The functor from the category of associative algebras to the category of Lie algebras is then realized as a morphism of PROP from LA to Alg.

6.5. Baker-Campbell-Hausdorff multiplication. The PROP LA is not wellsuited for the description of the adjoint construction, which assigns to a Lie algebra $\mathfrak{g}$ its universal enveloping algebra $U \mathfrak{g}$, however. The latter is isomorphic to the symmetric algebra $S \mathfrak{g}$ of $\mathfrak{g}$ as a vector space via the symmetrisation map $\sigma: S \mathfrak{g} \rightarrow$ $U \mathfrak{g}$. The multiplication $m$ on $U \mathfrak{g}$ can therefore be transported to $S \mathfrak{g}$ as the star product

$$
\star:=\sigma^{-1} \circ m \circ(\sigma \otimes \sigma)=\bigoplus_{k \leq i+j} m_{i, j}^{k},
$$

where $m_{i, j}^{k}: S^{i} \mathfrak{g} \otimes S^{j} \mathfrak{g} \rightarrow S^{k} \mathfrak{g}$ can be expressed in terms of the bracket on $\mathfrak{g}$ (see for example [6]). Indeed, $S \mathfrak{g}$ is spanned by the elements $Z^{k}, Z \in \mathfrak{g}, k \in \mathbb{N}$, and the corresponding generating series $\exp _{S}(v Z) \in S \mathfrak{g}[[v]]$ is mapped to $\exp _{U}(v Z) \in$ $U \mathfrak{g}[[v]]$ by $\sigma$. It follows that, for $X, Y \in \mathfrak{g}$,

$$
\left.\exp _{S}(t X) \star \exp _{S}(s Y)=\sigma^{-1}\left(\exp _{U}(t X) \exp _{U}(t X)\right)\right)=\exp _{S}(B(t X, s Y))
$$

where $B$ is the Baker-Campbell-Hausdorff Lie series. Thus,

$$
X^{i} \star Y^{j}=\left.\partial_{t}^{i} \partial_{s}^{j} \exp _{S}(B(t X, s Y))\right|_{t=s=0} .
$$

To describe the above procedure in terms of PROPs, ones needs to construct the subobjects $S^{k}[1] \subset[k]$ in LA. This requires replacing the PROP LA with its Karoubi envelope.

6.6. The Karoubi envelope. The Karoubi envelope of a category $\mathcal{C}$ is the category $\mathcal{C}^{\text {kar }}$ whose objects are pairs $(X, \pi)$, where $X \in \mathcal{C}$ and $\pi: X \rightarrow X$ is an idempotent. The morphisms in $\mathcal{C}^{\mathrm{kar}}$ are defined as

$$
\mathcal{C}^{\mathrm{kar}}((X, \pi),(Y, \rho))=\{f \in \mathcal{C}(X, Y) \mid \rho \circ f=f=f \circ \pi\}
$$

with $\operatorname{id}_{(X, \pi)}=\pi$. In particular,

$$
\mathcal{C}^{\mathrm{kar}}((X, \mathrm{id}),(Y, \mathrm{id}))=\mathcal{C}(X, Y)
$$

and the functor $\mathcal{C} \rightarrow \mathcal{C}^{\text {kar }}$, mapping $X \mapsto(X$, id $), f \mapsto f$, is fully faithful.

Every idempotent in $\mathcal{C}^{\mathrm{kar}}$ splits. Namely, if $q \in \mathcal{C}^{\mathrm{kar}}((X, \pi),(X, \pi))$ satisfies $q^{2}=q$, the maps

$$
i=q:(X, q) \rightarrow(X, \pi) \quad \text { and } \quad p=q:(X, \pi) \rightarrow(X, q)
$$

satisfy $i \circ p=q$ and $p \circ i=\operatorname{id}_{(X, q)}$. In particular, the Karoubi envelope $\mathcal{C}^{\text {kar }}$ of a PROP $\mathcal{C}$ contains the image of all the idempotents in $\mathrm{kS}_{n}$. This allows to consider the objects $S^{n}[1]:=\left([n], \operatorname{Sym}_{n}\right)$ for any $n \geq 0$, where $\operatorname{Sym}_{n}$ is the symmetriser $\frac{1}{n !} \sum_{\sigma \in \mathfrak{S}_{n}} \sigma$. If one further takes the closure $\underline{\mathcal{C}}$ of $\mathcal{C}^{\text {kar }}$ under possibly infinite inductive limits, then $\underline{\mathcal{C}}$ contains, in particular, the symmetric algebra $S[1]=\bigoplus_{n \geq 0} S^{n}[1]$. The construction of the universal enveloping algebra of a Lie algebra corresponds to a functor $U: A \mid g \rightarrow \underline{\mathrm{LA}}$ mapping $[1]_{\text {Alg }}$ to $S[1]_{\mathrm{LA}}$, and $m$ to $\bigoplus_{k \leq i+j} m_{i j}^{k}$. 
6.7. Etingof-Kazhdan quantisation. We consider the following categories

- the PROP LBA generated by morphisms [, ] : [2] $\rightarrow[1]$ and $\delta:[1] \rightarrow[2]$ such that $([1],[],, \delta)$ is a Lie bialgebra;

- the $\mathrm{k} \llbracket \hbar \rrbracket-$ linear category $\underline{\mathrm{LBA}} \llbracket \hbar \rrbracket$ with the same objects as $\underline{\mathrm{LBA}}$, and morphisms given by

$$
\operatorname{Hom}_{\underline{\underline{L A} \llbracket \llbracket \rrbracket}}(V, W)=\operatorname{Hom}_{\underline{\underline{L A A}}}(V, W) \llbracket \hbar \rrbracket ;
$$

- the PROP BA generated by morphisms $m:[2] \rightarrow[1], \iota:[0] \rightarrow[1], \Delta:$ $[1] \rightarrow[2]$, and $\varepsilon:[1] \rightarrow[0]$, such that $([1], m, \iota, \Delta, \varepsilon)$ is a bialgebra;

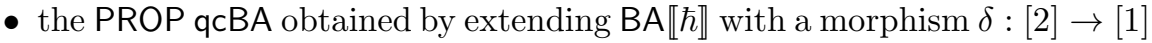
satisfying the relation $\Delta-(12) \circ \Delta=\hbar \delta$, and modding out the torsion ideal (cf. [11, §4.1]);

- the topological PROP QUE obtained by completing qcBA with respect to the ideal generated by $(\text { id }-\eta \circ \varepsilon)^{\otimes n} \circ \Delta^{(n)}$.

The quantisation functor can be described in this context [15, Thm. 1.2].

Theorem. There exists a universal quantisation functor

$$
Q: \text { QUE } \longrightarrow \underline{\text { LBA } \llbracket \hbar \rrbracket}
$$

such that

$$
\begin{aligned}
& Q[B]=S[\mathfrak{b}], \\
& Q(m)=m_{0} \\
& Q(\Delta)=\Delta_{0} \\
& \left.Q\left(\Delta-\Delta^{21}\right)\right|_{[\mathfrak{b}]}=\hbar \delta \quad \bmod \hbar^{2} .
\end{aligned}
$$

where $[B]$ and $[\mathfrak{b}]$ the generating objects in QUE and LBA, respectively, and $m_{0}, \Delta_{0}$ are the product and coproduct of the enveloping algebra $U[\mathfrak{b}]$.

The functor $Q$ depends upon the choice of an associator $\Phi$, and is equivalent to the construction of a Hopf algebra structure on $U[\mathfrak{b}]=S[\mathfrak{b}]$, which lifts to the category LBA $\llbracket \hbar \rrbracket$ the construction described in $\S 2.16$. We outline this lift in $\S 6.8-$ 6.16 below.

6.8. Drinfeld-Yetter modules. Let $\mathrm{DY}_{[\mathfrak{b}]}$ be the strict, symmetric tensor category of Drinfeld-Yetter modules over the Lie bialgebra [b] in LBA $\llbracket \hbar \rrbracket$. For any

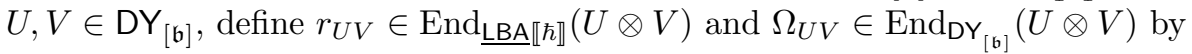

$$
r_{U V}=\pi_{U} \otimes \mathrm{id} \circ(12) \circ \mathrm{id} \otimes \pi_{V}^{*} \quad \text { and } \quad \Omega_{U V}=r_{U V}+r_{V, U}^{21} .
$$

Let $\Phi$ be a Lie associator, and $D Y_{[\mathfrak{b}]}^{\Phi}$ the category $\mathrm{DY}_{[\mathfrak{b}]}$ with deformed commutativity and associativity respectively given by

$$
\beta_{U V}=(12) \circ \exp \left(\frac{\hbar}{2} \Omega_{U V}\right) \quad \text { and } \quad \Phi_{U V W}=\Phi\left(\hbar \Omega_{U V}, \hbar \Omega_{V W}\right) .
$$

6.9. The Verma module $\left[M_{-}\right]$. The Verma modules $M_{-}, M_{+}^{\vee}$ may be lifted to Drinfeld-Yetter modules $\left[M_{-}\right],\left[M_{+}^{\vee}\right]$ over $[\mathfrak{b}]$ in LBA. $\left[M_{-}\right]$is obtained as follows.

- As an object, $\left[M_{-}\right]=S[\mathfrak{b}]$.

- The action $\pi$ of $[\mathfrak{b}]$ on $\left[M_{-}\right]$is given by the multiplication map

$$
\bigoplus_{0 \leq j \leq i+1} m_{1, i}^{j}:[\mathfrak{b}] \otimes S[\mathfrak{b}] \rightarrow S[\mathfrak{b}]
$$

obtained from the Baker-Campbell-Hausdorff series described in 6.5. 
- The coaction $\pi^{*}$ of $[\mathfrak{b}]$ is uniquely determined by requiring that it be

- trivial on $S^{0}[\mathfrak{b}]$;

- compatible with the action of $[\mathfrak{b}]$ via the relation

$$
\pi^{*} \circ \pi=\mathrm{id} \otimes \pi \circ(12) \circ \mathrm{id} \otimes \pi^{*}-\mathrm{id} \otimes \pi \circ \delta \otimes \mathrm{id}+[,] \otimes \mathrm{id} \circ \mathrm{id} \otimes \pi^{*} .
$$

Specifically, the above requirements uniquely determine a map $\pi^{*}:\left[M_{-}\right] \rightarrow$ $[\mathfrak{b}] \otimes\left[M_{-}\right]$, by induction on the $\mathbb{N}$-grading of $S[\mathfrak{b}]$. A diagrammatic computation then shows that $\pi_{*}$ is a coaction of $[\mathfrak{b}]$.

\subsection{Universal property of $\left[M_{-}\right]$.}

Lemma. For any $[V] \in \mathrm{DY}_{[\mathfrak{b}]}$, there is an isomorphism

$$
\operatorname{Hom}_{[\mathfrak{b}]}^{[\mathfrak{b}]}\left(\left[M_{-}\right],[V]\right) \simeq \operatorname{Hom}^{[\mathfrak{b}]}([0],[V])
$$

which is natural in $[V]$.

Proof. We proceed as in the case of the quantum Verma module $M_{B}$ (cf. §4.1). $\left[M_{-}\right]$is a unital algebra, with multiplication given by the BCH series $m_{\mathfrak{b}}$, and unit given by the inclusion $\iota:[0] \rightarrow\left[M_{-}\right] .\left[M_{-}\right]$is therefore a module over itself, with $\bar{\pi}_{\left[M_{-}\right]}=m_{\mathfrak{b}}$, and the action of $[\mathfrak{b}]$ on $\left[M_{-}\right]$is recovered by restriction to $[\mathfrak{b}]$, i.e., $\pi_{\left[M_{-}\right]}=m_{\mathfrak{b}} \circ \iota_{\mathfrak{b}} \otimes \mathrm{id}_{\left[M_{-}\right]}$where $\iota_{\mathfrak{b}}$ is the canonical inclusion $\iota_{\mathfrak{b}}:[\mathfrak{b}] \rightarrow\left[M_{-}\right]$. Similarly, any action $\pi_{[V]}:[\mathfrak{b}] \otimes[V] \rightarrow[V]$ extends to an action $\bar{\pi}_{[V]}:\left[M_{-}\right] \otimes[V] \rightarrow$ $[V]$ of $\left(\left[M_{-}\right], m_{\mathfrak{b}}, \iota\right)$ on $[V]$, which is defined on $S^{n}[\mathfrak{b}]$ by $\pi_{[V]}^{(n)} \circ$ Sym ${ }_{n}$, where $\pi_{[V]}^{(n)}$ denotes the $n$th iterated action.

The description of (6.3) is the following. In one direction, for any Drinfeld-Yetter morphism $g:\left[M_{-}\right] \rightarrow[V]$, one obtains a $[\mathfrak{b}]$-comodule map $\phi(g)=g \circ \iota:[0] \rightarrow[V]$. Conversely, for any morphism of comodules $f:[0] \rightarrow[V]$, one defines a module $\operatorname{map} \psi(f)=\bar{\pi}_{[V]} \circ \mathrm{id}_{\left[M_{-}\right]} \otimes f:\left[M_{-}\right] \rightarrow[V]$. One has $\psi \circ \phi(g)=g$, since $\left[M_{-}\right]$is free of rank one on itself (i.e., $\left.\bar{\pi}_{\left[M_{-}\right]} \circ \mathrm{id}_{\left[M_{-}\right]} \otimes \iota=\operatorname{id}_{\left[M_{-}\right]}\right)$, and $\phi \circ \psi(f)=f$, since $\bar{\pi}_{[V]}$ is an action map (i.e., $\left.\bar{\pi}_{[V]} \circ \iota \otimes \mathrm{id}_{[V]}=\operatorname{id}_{[V]}\right)$.

The same argument shows that, if $\mathfrak{b}$ is a Lie bialgebra, and $\mathcal{G}_{\mathfrak{b}}:$ LBA $\rightarrow$ Vect $_{k}$ a symmetric tensor functor such that $\mathcal{G}_{\mathfrak{b}}[\mathfrak{b}]=\mathfrak{b}$, then $\mathcal{G}_{\mathfrak{b}}\left(\left[M_{-}\right]\right)$is a solution of the universal property

$$
\operatorname{Hom}_{\mathfrak{b}}^{\mathfrak{b}}\left(\mathcal{G}_{\mathfrak{b}}\left(\left[M_{-}\right]\right), V\right) \simeq \operatorname{Hom}^{\mathfrak{b}}(\mathrm{k}, V)
$$

for any $V \in \mathrm{DY}_{\mathfrak{b}}$. In particular, $\mathcal{G}_{\mathfrak{b}}\left(\left[M_{-}\right]\right)$is the Drinfeld-Yetter module $M_{-}=$ $\operatorname{Ind}_{\mathfrak{b}^{*}}^{\mathfrak{g}_{\mathfrak{b}}} \mathbf{k}$ over $\mathfrak{b}$. Alternatively, the result follows by identifying $\mathcal{G}_{\mathfrak{b}}\left(\left[M_{-}\right]\right)$and $M_{-}$ as modules over $[\mathfrak{b}]$, and using the fact that the coaction of $[\mathfrak{b}]$ on $M_{-}$is uniquely determined.

6.11. Dualising Drinfeld-Yetter modules. The PROPic description of the module $M_{+}^{\vee}$ given in $\S 6.12$ below relies on the following considerations.

Let $V$ be a Drinfeld-Yetter module over a Lie bialgebra $(\mathfrak{b},[],, \delta)$ with left action and right coaction

$$
\pi_{V}: \mathfrak{b} \otimes V \rightarrow V \quad \text { and } \quad \pi_{V}^{*}: V \rightarrow \mathfrak{b} \otimes V .
$$

Then, the dual vector space $V^{*}$ is a Drinfeld-Yetter module over the dual (topological) Lie bialgebra $\left(\mathfrak{b}^{*}, \delta^{t},[,]^{t}\right)$, with action and coaction given by

$$
\rho_{V^{*}}=-\left(\pi_{V}^{*}\right)^{t}: \mathfrak{b}^{*} \otimes V^{*} \rightarrow V^{*} \quad \text { and } \quad \rho_{V^{*}}^{*}=-\pi_{V}^{t}: V^{*} \rightarrow \mathfrak{b}^{*} \otimes V^{*} .
$$


If $\mathfrak{b}$ if finite-dimensional, the corresponding functor $\mathrm{DY}_{\mathfrak{b}} \rightarrow \mathrm{DY}_{\mathfrak{b} *}$ is readily seen to coincide with the duality enodfunctor on $\operatorname{Rep}\left(\mathfrak{g}_{\mathfrak{b}}\right)$, via the identifications $\mathrm{DY}_{\mathfrak{b}}=\operatorname{Rep}\left(\mathfrak{g}_{\mathfrak{b}}\right)=\mathrm{DY}_{\mathfrak{b}^{*}}$.

To lift this to the PROPic setting, notice the following.

- Let $\Theta:$ LBA $\rightarrow$ LBA be the strict, contravariant symmetric tensor functor mapping $[n]$ to $[n]$, a permutation $\sigma \in \operatorname{LBA}([n],[n])$ to $\sigma^{-1}$, and permuting the bracket [, ] : [2] $\rightarrow[1]$ and the cobracket $\delta:[1] \rightarrow[2]$. Since $\Theta \circ \Theta$ is the identity functor, $\Theta$ is an isomorphism of LBA onto its opposite category.

- $\Theta$ is a PROPic lift of the (symmetric, tensor) duality functor D : Vect ${ }_{k} \rightarrow$ Vect $_{k}$ in the following sense. If $\mathcal{G}_{\mathfrak{b}}:$ LBA $\rightarrow$ Vect $_{k}$ is a symmetric tensor functor such that $\mathcal{G}_{\mathfrak{b}}[1]=\mathfrak{b}$, the functor $\mathcal{G}_{\mathfrak{b} *}=D \circ \mathcal{G}_{\mathfrak{b}} \circ \Theta$ maps [1] to the Lie bialgebra $\mathfrak{b}^{*}$ and, evidently, makes the following a commutative diagram

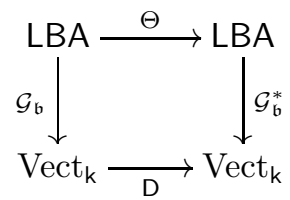

- If $\left(V, \pi_{V}, \pi_{V}^{*}\right) \in \mathrm{DY}_{\mathfrak{b}}$ is PROPic, that is of the form $\mathcal{G}_{\mathfrak{b}}\left([V], \pi, \pi^{*}\right)$, where $[V] \in \mathrm{DY}_{[\mathfrak{b}]}$, then so is $V^{*}$ as a Drinfeld-Yetter module over $\mathfrak{b}^{*}$. Specifically,

$$
\left(V^{*}, \rho_{V^{*}}, \rho_{V^{*}}^{*}\right)=\mathcal{G}_{\mathfrak{b}^{*}}\left(\Theta[V], \Theta\left(-\pi^{*}\right), \Theta(-\pi)\right)
$$

as follows from (6.4) and (6.5).

6.12. The Verma module $\left[M_{+}^{\vee}\right]$. Let $\mathfrak{b}$ be a finite-dimensional Lie bialgebra. Since the action and coaction of $\mathfrak{b}^{*}$ on the Verma module $M_{+}=\operatorname{Ind}_{\mathfrak{b}}^{\mathfrak{g}_{\mathfrak{b}}} \mathrm{k}$ are described by the same formulae as those of $\mathfrak{b}$ on $M_{-}=\operatorname{Ind}_{\mathfrak{b}^{*}}^{\mathfrak{g}_{\mathfrak{b}}} \mathbf{k},(6.4)$ may be used to describe $M_{+}^{\vee}=M_{+}^{*}$ as a Drinfeld-Yetter module over $\mathfrak{b}=\left(\mathfrak{b}^{*}\right)^{*}$. It then follows from (6.6) that the Drinfeld-Yetter module $M_{+}^{\vee}$ possesses a lift to the PROP $\underline{\text { LBA }}$, given by

$$
\left(\left[M_{+}^{\vee}\right], \pi_{+}, \pi_{+}^{*}\right)=\left(\Theta\left[M_{-}\right], \Theta\left(-\pi_{-}^{*}\right), \Theta\left(-\pi_{-}\right)\right),
$$

where $\pi_{-}, \pi_{-}^{*}$ are the action and coaction on $\left[M_{-}\right]$. In other words, $\left[M_{+}^{\vee}\right]$ is obtained from $\left[M_{-}\right]$by exchanging action and coaction, permuting brackets and cobrackets, reversing the order of operations, and applying a minus sign, as stated in [15, $\S 1.4]$. Note in particular that the application of the functor $\Theta$ turns the ind-object $\left[M_{-}\right]=S[\mathfrak{b}]$ into the pro-object $\left[M_{+}^{\vee}\right]=\widehat{S[\mathfrak{b}]}$.

Let now $\mathfrak{b}$ an arbitrary Lie bialgebra, and $\mathcal{G}_{\mathfrak{b}}:$ LBA $\rightarrow$ Vect $_{\mathrm{k}}$ a symmetric tensor functor such that $\mathcal{G}_{\mathfrak{b}}[\mathfrak{b}]=\mathfrak{b}$. We show in $\S 6.13$ that $\mathcal{G}_{\mathfrak{b}}\left[M_{+}^{\vee}\right]$ satisfies the same universal property as $M_{+}^{\vee}$. It follows that $\left[M_{+}^{\vee}\right]$ is PROPic lift of the module $M_{+}^{\vee}$ for any $\mathfrak{b}$. Alternatively, to show that $\mathcal{G}_{\mathfrak{b}}\left[M_{+}^{\vee}\right]=M_{+}^{\vee}$ in $\mathrm{DY} \mathfrak{b}$, it suffices to prove that their duals are equal in $\mathrm{DY}_{\mathfrak{b}^{*}}$. By (6.5) and Definition (6.7),

$$
\mathcal{G}_{\mathfrak{b}}\left[M_{+}^{\vee}\right]^{*}=\mathcal{G}_{\mathfrak{b} *}\left[M_{-}\right]=\widehat{M_{+}}
$$

where $\widehat{M_{+}}$is the completion of the (algebraic) symmetric algebra of $\mathfrak{b}^{*}$ with respect to the weak topology. The action of $\mathfrak{b}^{*}$ on $\widehat{M_{+}}$is given by the $\mathrm{CBH}$ product, and its coaction is uniquely determined by the fact that it kills the generating vector, and is compatible with the action. Since $\left(M_{+}^{\vee}\right)^{*}$ also satisfies these properties, the two coincide. 


\subsection{Universal property of $\left[M_{+}^{\vee}\right]$.}

\section{Lemma.}

(i) For any $[V] \in \mathrm{DY}_{[\mathfrak{b}]}$, there is an isomorphism

$$
\operatorname{Hom}_{[\mathfrak{b}]}^{[\mathfrak{b}]}\left([V],\left[M_{+}^{\vee}\right]\right) \simeq \operatorname{Hom}^{[\mathfrak{b}]}([V],[0])
$$

which is natural in $[V]$.

(ii) If $\mathfrak{b}$ is a Lie bialgebra, and $\mathcal{G}_{\mathfrak{b}}:$ LBA $\rightarrow$ Vect $_{\mathfrak{k}}$ a symmetric tensor functor such that $\mathcal{G}_{\mathfrak{b}}[\mathfrak{b}]=\mathfrak{b}$, then $\mathcal{G}_{\mathfrak{b}}\left(\left[M_{+}^{\vee}\right]\right)$ is a solution of the universal property

$$
\operatorname{Hom}_{\mathfrak{b}}^{\mathfrak{b}}\left(V, M_{+}^{\vee}\right) \simeq \operatorname{Hom}^{\mathfrak{b}}(V, \mathbf{k})
$$

for any $V \in \mathrm{DY}_{\mathfrak{b}}$.

Proof. We argue as in the case of the quantum Verma module $M_{B}^{\vee}$ (cf. $\S 4.2-$ 4.4). $\left[M_{+}^{\vee}\right]=\widehat{S}[\mathfrak{b}]$ is a counital coalgebra with comultiplication $\Delta_{\mathfrak{b}}=\Theta\left(m_{\mathfrak{b}}\right)$, and unit given by the canonical projection $\varepsilon=\Theta(\iota):\left[M_{+}^{\vee}\right] \rightarrow[0]$. Therefore it is a comodule over itself, with $\bar{\pi}_{\left[M_{+}^{\vee}\right]}^{*}=\Theta\left(m_{\mathfrak{b}}\right)^{\text {op }}$, and the coaction of [b] on $\left[M_{-}\right]$ is recovered by projection to $[\mathfrak{b}]$, i.e., $\pi_{\left[M_{-}\right]}^{*}=p_{\mathfrak{b}} \otimes \mathrm{id}_{\left[M_{+}^{\vee}\right]} \circ \Delta_{\mathfrak{b}}^{o p}$ where $p_{\mathfrak{b}}$ is the canonical projection $p_{\mathfrak{b}}=\Theta\left(\iota_{\mathfrak{b}}\right):\left[M_{+}^{\vee}\right] \rightarrow[\mathfrak{b}]$. Similarly, any coaction $\pi_{[V]}^{*}:[V] \rightarrow$ $[\mathfrak{b}] \otimes[V]$ extends to a coaction of $\left(\left[M_{+}^{\vee}\right], \Delta_{\mathfrak{b}}, \varepsilon\right)$ on $[V], \bar{\pi}_{[V]}^{*}:[V] \rightarrow\left[M_{+}^{\vee}\right] \otimes[V]$, given by $\sum_{n \geqslant 0} \operatorname{Sym}_{n} \circ\left(\pi^{*}\right)_{[V]}^{(n)}$, where $\left(\pi^{*}\right)_{[V]}^{(n)}$ denotes the $n$th iterated coaction.

(i) For any Drinfeld-Yetter morphism $g:[V] \rightarrow\left[M_{+}^{\vee}\right]$, one obtains a [b]-module $\operatorname{map} \phi(g)=\varepsilon \circ g:[V] \rightarrow[0]$. Conversely, for any morphism of modules $f:[V] \rightarrow[0]$, one defines a module map $\psi(f)=\circ \operatorname{id}_{\left[M_{+}^{\vee}\right]} \otimes f \circ \bar{\pi}_{[V]}^{*}:[V] \rightarrow\left[M_{+}^{\vee}\right]$. One has $\psi \circ \phi(g)=g$, since $\left[M_{+}^{\vee}\right]$ is cofree of rank one on itself, i.e., $\operatorname{id}_{\left[M_{+}^{\vee}\right]} \otimes \varepsilon \circ \bar{\pi}_{\left[M_{+}^{\vee}\right]}^{*}=$ $\operatorname{id}_{\left[M_{+}^{\vee}\right]}$, and $\phi \circ \psi(f)=f$, since $\bar{\pi}_{[V]}^{*}$ is a coaction map (i.e., $\left.\varepsilon \otimes \operatorname{id}_{[V]} \circ \bar{\pi}_{[V]}^{*}=\operatorname{id}_{[V]}\right)$. (ii) Follows by the same argument.

6.14. Fiber functor. There is a tensor structure on the tautological forgetful functor $\mathrm{f}_{[\mathfrak{b}]}: \mathrm{DY}_{[\mathfrak{b}]}^{\Phi} \rightarrow \underline{\mathrm{LBA}} \llbracket \hbar \rrbracket$. The latter is obtained by imitating the construction of the fiber functor $F_{\mathfrak{b}}$ defined in $\S 2.15$, once the latter has been identified with the forgetful functor $\mathrm{f}_{\mathfrak{b}}: \mathrm{DY}_{\mathfrak{b}}^{\Phi} \rightarrow$ Vect $_{\mathrm{k} \llbracket \hbar \rrbracket} \cdot{ }^{9}$

Specifically, the modules $\left[M_{-}\right],\left[M_{+}^{\vee}\right]$ satisfy, for any $V, W \in \mathrm{DY}_{[\mathfrak{b}]}$

$$
\operatorname{Hom}_{[\mathfrak{[ b ]}]}^{[\mathfrak{b}]}\left(\left[M_{-}\right] \otimes V^{0},\left[M_{+}^{\vee}\right] \otimes W\right) \simeq \operatorname{Hom}_{\underline{\underline{L B A}} \llbracket \hbar \rrbracket}(V, W)
$$

where $V^{0}$ denotes the object $V$ with trivial action and coaction. Let $\psi_{V}:\left[M_{-}\right] \otimes$ $V^{0} \rightarrow\left[M_{+}^{\vee}\right] \otimes V$ be the morphism corresponding to $\operatorname{id}_{V}$ under (6.8), and let $\eta_{V} \in$ $\operatorname{Hom}_{\underline{L B A} \llbracket \hbar \rrbracket}\left(V,\left[M_{+}^{\vee}\right] \otimes V\right)$ be defined by $\eta_{V}=\psi_{V} \circ 1_{-} \otimes \operatorname{id}_{V}$, where $1_{-}:[0] \rightarrow\left[M_{-}\right]$ is the canonical inclusion in $S[\mathfrak{b}]$. by

The tensor structure on the forgetful functor from $D Y_{[\mathfrak{b}]}^{\Phi}$ to $\underline{L B A} \llbracket \hbar \rrbracket$ is then given

$$
J_{V W}=\left(1_{+} \otimes \mathrm{id}\right)^{\otimes 2} \circ A_{\Phi} \circ \eta_{V} \otimes \eta_{W} \in \operatorname{End}_{\underline{\underline{L A}} \llbracket \hbar \rrbracket}(V \otimes W),
$$

where $V, W \in \mathrm{DY}_{[\mathfrak{b}]}^{\Phi}$, and $A_{\Phi}$ is the composition of associativity and commutativity constraints defined in $\S 2.15$.

\footnotetext{
${ }^{9}$ The functor $F_{\mathfrak{b}}(V)=\operatorname{Hom}_{\mathfrak{b}}^{\mathfrak{b}}\left(M_{-}, M_{+}^{\vee} \widehat{\otimes} V\right)$ itself is not PROPic since, for $V \in \mathrm{DY}_{[\mathfrak{b}]}^{\Phi}$, $\operatorname{Hom}_{[\mathfrak{b}]}^{[\mathfrak{b}]}\left(\left[M_{-}\right],\left[M_{+}^{\vee}\right] \otimes V\right)$ is a vector space, not an object in $\underline{\mathrm{LBA}} \llbracket \hbar \rrbracket$.
} 
6.15. Quantisation of $U[\mathfrak{b}]$. The object [ $\left.M_{-}\right]$has a structure of algebra and coalgebra with unit $\widetilde{\iota}$ and counit $\tilde{\epsilon}$ given by the inclusion of and the projection to [0], respectively, and with product and coproduct given by the maps

$$
\widetilde{m}=1_{+} \otimes 1_{+} \otimes \mathrm{id} \circ \mathrm{id} \otimes \psi_{\left[M_{-}\right]} \circ \Phi \circ \eta_{\left[M_{-}\right]} \otimes \mathrm{id} \quad \text { and } \quad \widetilde{\Delta}=J_{\left[M_{-}\right],\left[M_{-}\right]}^{-1} \circ \Delta_{0},
$$

where $1_{+}$is the canonical projection of $\left.\left[M_{+}^{\vee}\right]=\widehat{S[\mathfrak{b}}\right]$ onto [0], and $\Delta_{0}$ is the standard coproduct on $\left[M_{-}\right]=S[\mathfrak{b}]$. Moreover, $\left[M_{-}\right]$acts on any $V \in \mathrm{DY}_{[\mathfrak{b}]}^{\Phi}$, with action

$$
\rho_{V}=1_{+} \otimes 1_{+} \otimes \mathrm{id} \circ \mathrm{id} \otimes \psi_{V} \circ \Phi \circ \eta_{\left[M_{-}\right]} \otimes \mathrm{id}
$$

satisfying $\rho_{V} \circ \widetilde{m} \otimes \mathrm{id}=\rho_{V} \circ\left(\mathrm{id} \otimes \rho_{V}\right)$ and

$$
\rho_{V \otimes W}=J_{V W} \circ \rho_{V} \otimes \rho_{W} \circ(23) \circ \widetilde{\Delta} \otimes J_{V W}^{-1} .
$$

Since the bialgebra structure is a deformation of that on $U[\mathfrak{b}]$, it follows that $\left(\left[M_{-}\right], \widetilde{m}, \widetilde{\Delta}\right)$ admits a Hopf algebra structure (with antipode $\widetilde{S}$ ), and we set

$$
Q([B], m, \iota, \Delta, \epsilon, S)=\left(\left[M_{-}\right], \widetilde{m}, \widetilde{\iota}, \widetilde{\Delta}, \widetilde{\epsilon}, \widetilde{S}\right) .
$$

It is easy to check that $Q[B]$ is a quantisation of the Lie bialgebra $[\mathfrak{b}]$ and gives rise to a functor $Q:$ QUE $\rightarrow \underline{\mathrm{LBA}} \llbracket \hbar \rrbracket$.

6.16. Tannakian lift of $\mathrm{f}_{[\mathfrak{b}]} \cdot\left[M_{-}\right]$coacts on any $V \in \mathrm{DY}_{[\mathfrak{b}]}^{\Phi}$, with coaction

$$
\rho_{V}^{*}=R^{J} \circ \widetilde{\iota} \otimes \mathrm{id}_{V}
$$

where $R^{J}$ is the twisted $R$-matrix defined by $R^{J}=(12) J_{V, W}^{-1} \beta_{V, W} J_{V, W}$. The fiber functor $\mathrm{f}_{[\mathfrak{b}]}$ then lifts to a braided monoidal functor $\widetilde{f}_{[\mathfrak{b}]}: D Y_{[\mathfrak{b}]}^{\Phi} \rightarrow D Y_{Q[B]}^{\text {adm }}$, where the latter is the category of Drinfeld-Yetter modules over the Hopf algebra $Q[B]$ in $\underline{\mathrm{LBA}} \llbracket \hbar \rrbracket$.

The functor $\widetilde{f}_{[\mathfrak{b}]}$ is a propic version of the Tannakian equivalence $\widetilde{F}_{\mathfrak{b}}$. More precisely, any symmetric tensor functor $\mathcal{G}_{\mathfrak{b}}:$ LBA $\rightarrow$ Vect $_{k}$, which maps $[\mathfrak{b}]$ to a Lie bialgebra $\mathfrak{b}$ gives rise to functors

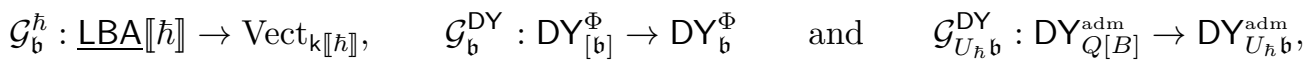

where $\mathcal{G}_{\mathfrak{b}}^{\hbar}([\mathfrak{b}])=\mathfrak{b} \llbracket \hbar \rrbracket, \mathcal{G}_{\mathfrak{b}}^{\hbar}(\mu)=\mu, \mathcal{G}_{\mathfrak{b}}^{\hbar}(\delta)=\delta$, and $\mathrm{DY}_{Q[B]}^{\text {adm }}$ is the PROP defined in $\S 6.17 .1$, such that

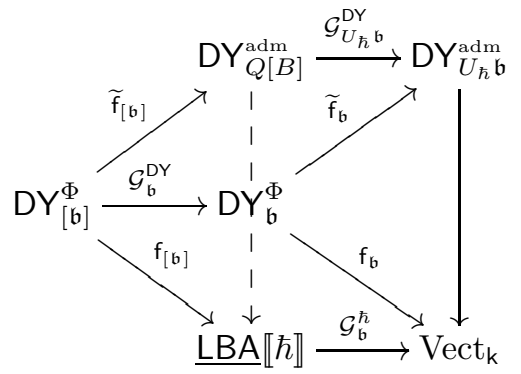

is a commutative diagram of tensor functors, where $\widetilde{f}_{\mathfrak{b}} \cong \widetilde{F}_{\mathfrak{b}}$ is obtained through the isomorphism $f_{\mathfrak{b}} \cong F_{\mathfrak{b}}$. 
6.17. The equivalence $\widetilde{f}_{\mathfrak{b}}$. We now sketch an alternative proof of Theorem 2.24 that the functor $\widetilde{f}_{\mathfrak{b}}: D Y_{\mathfrak{b}}^{\Phi} \rightarrow D Y_{U_{\mathfrak{b} \mathfrak{b}}}^{\text {adm }}$ is a braided tensor equivalence.

It was shown in [11, Cor. 6.4] that the existence of a quantisation functor $Q:$ QUE $\rightarrow$ LBA $\llbracket \hbar \rrbracket$, together with Hensel's Lemma, imply that $Q$ gives rise to an isomorphism of QUE and the PROP UE $\mathrm{CP}_{\mathrm{cP}} \llbracket \hbar \rrbracket$ of co-Poisson universal enveloping algebras. We shall adopt a similar strategy to show that $f_{\mathfrak{b}}$ is an equivalence.

6.17.1. Consider the colored PROPs (see §7.2) DY LBA $_{\text {and }}$ DY QUE describing, respectively, a Drinfeld-Yetter module over a Lie bialgebra, and an admissible DrinfeldYetter module over a quantised enveloping algebra.

The PROP DY ${ }_{\text {LBA }}$ is generated by a Lie bialgebra object $[\mathfrak{b}]$, together with an object $[V]$ endowed with the structure of Drinfeld-Yetter module over $[\mathfrak{b}]$. There is a canonical equivalence of categories

$$
\mathrm{DY}_{\mathfrak{b}}^{\Phi} \simeq\left\{\mathcal{F} \in \operatorname{Fun}_{\mathbf{b}}^{\otimes}\left(\mathrm{DY}_{\mathrm{LBA}}, \operatorname{Vect}_{\mathrm{k}}\right) \mid \mathcal{F}[\mathfrak{b}]=\mathfrak{b}\right\}
$$

The PROP DY QUE is generated by a QUE object $[B]$, together with a DrinfeldYetter module $[V]$ over $[B]$, whose coaction factors through the QFSH subalgebra of $[B]$. This last condition is encoded by requiring the existence of a map $\Xi_{[V]}$ : $[V] \rightarrow[B] \otimes[V]$ such that

$$
\left(\mathrm{id}-\eta_{[B]} \circ \epsilon_{[B]}\right) \otimes \operatorname{id}_{[V]} \circ \pi_{[V]}^{*}=\hbar \Xi_{[V]}
$$

and modding out the torsion ideal. In particular, it follows, as in the proof of Proposition 2.21,

$$
\left(\left(\mathrm{id}-\eta_{[B]} \circ \epsilon_{[B]}\right)^{\otimes n} \circ \Delta^{(n)}\right) \otimes \operatorname{id}_{[V]} \circ \pi_{[V]}^{*}=\hbar^{n} \Xi_{[V]}^{(n)},
$$

where $\Xi_{[V]}^{(n)}:[V] \rightarrow[B]^{\otimes n} \otimes[V]$ is the $n$th iteration of $\Xi_{[V]}$. Therefore, for any quantised universal enveloping algebra $B$, there is an equivalence

$$
\mathrm{DY}_{B}^{\text {adm }} \simeq\left\{\mathcal{F} \in \operatorname{Fun}_{\mathbf{b}}^{\otimes}\left(\text { DY }_{\text {QUE }}^{\text {adm }}, \text { Vect }_{\mathrm{k}}\right) \mid \mathcal{F}[B]=B\right\} .
$$

6.17.2. The formulae from $\S 6.15$ and $\S 6.16$ allow to extend the quantisation functor $Q:$ QUE $\rightarrow$ LBA $\llbracket \hbar \rrbracket$ to a tensor functor $\widetilde{Q}: \mathrm{DY}_{\mathrm{QUE}}^{\mathrm{adm}} \rightarrow \mathrm{DY} \mathrm{LBA}_{\mathrm{L}} \llbracket \hbar \rrbracket$. Under the equivalences $(6.9)-(6.10)$, the braided tensor functor $\widetilde{f}_{\mathfrak{b}}$ corresponds to the pullback of $\widetilde{Q}$.

Much like $Q$, the functor $\widetilde{Q}$ is not essentially surjective since DY LBA $\llbracket \rrbracket$ has many more objects. To remedy this, one can introduce, by analogy with [11, Cor. 6.4], a PROP DY $\mathrm{UE}_{\mathrm{CP}}$ describing the notion of Drinfeld-Yetter module over a co-Poisson universal enveloping algebra.

The presentation of $D Y_{U E_{c P}}^{a d m}$ is tailored to describe the subcategory of DY ${ }_{L B A}$ generated by the $\mathrm{UE}_{\mathrm{cP}}-$ module $S[\mathfrak{b}]$ and the object $[V]$. The latter is endowed with an action map $\mu_{[V]}: S[\mathfrak{b}] \otimes[V] \rightarrow[V]$ given by

$$
\mu_{[V]}=\bigoplus_{n \geq 0} \pi_{[V]}^{(n)} \circ \operatorname{Sym}_{n} \otimes \operatorname{id}_{[V]},
$$

where $\operatorname{Sym}_{n}: S^{n}[\mathfrak{b}] \rightarrow[\mathfrak{b}]^{\otimes n}$ is the inclusion, and the maps $\pi_{[V]}^{(n)}:[\mathfrak{b}]^{\otimes n} \otimes[V] \rightarrow[V]$ are recursively defined by $\pi_{[V]}^{(1)}=\pi_{[V]}$, with $\pi_{[V]}$ the action of $[\mathfrak{b}]$ on $[V]$, and, for 
$n \geq 2, \pi_{[V]}^{(n)}=\pi_{[V]} \circ \mathrm{id}_{[\mathfrak{b}]} \otimes \pi_{[V]}^{(n-1)}$. It is also endowed with a family of coaction maps $\xi_{[V]}^{(n)}:[V] \rightarrow S[\mathfrak{b}] \otimes[V]$

$$
\xi_{[V]}^{(n)}=i_{n} \circ \operatorname{Sym}_{n} \otimes \operatorname{id}_{[V]} \circ\left(\pi_{[V]}^{*}\right)^{(n)},
$$

where $\left(\pi_{[V]}^{*}\right)^{(n)}:[V] \rightarrow[\mathfrak{b}]^{\otimes n} \otimes[V]$ is the iterated coaction, $\operatorname{Sym}_{n}:[\mathfrak{b}]^{\otimes n} \rightarrow S^{n}[\mathfrak{b}]$ the projection, and $i_{n}: S^{n}[\mathfrak{b}] \hookrightarrow S[\mathfrak{b}]$ the inclusion. The relations satisfied by $\mu_{[V]}$ and $\xi_{[V]}^{(n)}$ are deduced from those in DY LBA.

6.17.3. The functor $\widetilde{Q}$ restricts to a morphism of PROPs DY $Y_{Q U E}^{\mathrm{adm}} \stackrel{\sim}{\longrightarrow} \mathrm{DY}_{U \mathrm{UE}_{c \mathrm{P}}}^{\mathrm{adm}} \llbracket \hbar \rrbracket$ which is essentially surjective by construction and fully faithful by Hensel's Lemma. Finally, since the category of Drinfeld-Yetter $\mathfrak{b}$-modules is equivalent to the category of $\mathrm{DY}_{\mathrm{UE}_{\mathrm{cP}}}^{\mathrm{adm}}-$ modules, i.e.,

$$
\mathrm{DY}_{\mathfrak{b}} \simeq\left\{\mathcal{F} \in \mathrm{Fun}_{\mathrm{b}}^{\otimes}\left(\mathrm{DY}_{\mathrm{UE}}^{\mathrm{adm}}, \operatorname{Vect}\right) \mid \mathcal{F}(S[\mathfrak{b}])=S \mathfrak{b}\right\}
$$

one concludes that the pullback of $\widetilde{Q}$, and therefore the functor $\widetilde{f}_{\mathfrak{b}}$ from $D Y_{\mathfrak{b}}^{\Phi}$ to $\mathrm{DY}_{U_{\hbar} \mathfrak{b}}$, is an equivalence of braided tensor categories.

\section{UNIVERSAL RELATIVE CONSTRUCTIONS}

In this section, we show that the quantisations of the Verma modules $L_{-}, N_{+}^{\vee}$ are isomorphic to their quantum counterparts $L_{-}^{\hbar},\left(N_{+}^{\vee}\right)^{\hbar}$, thus proving Theorem 4.13. We also show that the constructions of Sections $\S 3, \S 4$ and $\S 5$ can be realised in the context of PROPs, and are therefore functorial with respect to morphisms of split pairs of Lie bialgebras.

7.1. Colored PROPs. The definition of PROP is easily generalised to allow a larger set of generating objects. A colored PROP P is a k-linear, strict, symmetric monoidal category whose objects are finite sequences over a set $A$, i.e.,

$$
\operatorname{Obj}(\mathrm{P})=\coprod_{n \geq 0} \mathrm{~A}^{n}
$$

with tensor product given by concatenation of sequences, and tensor unit given by the empty sequence. The notion of module over a colored PROP is easily generalised from $\S 6.1$.

7.2. PROP for split pairs of Lie bialgebras. Let PLBA be the PROP generated by two Lie bialgebra objects $[\mathfrak{a}],[\mathfrak{b}]$ and Lie bialgebra morphisms $i:[\mathfrak{a}] \rightarrow[\mathfrak{b}]$, $p:[\mathfrak{b}] \rightarrow[\mathfrak{a}]$ satisfying $p \circ i=\operatorname{id}_{[\mathfrak{a}]}$.

The PROP PLBA is endowed with a pair of (strict) tensor functors

$$
\mathcal{G}_{[\mathfrak{b}]}, \mathcal{G}_{[\mathfrak{a}]}: \text { LBA } \rightarrow \text { PLBA } \quad \text { given by } \quad \mathcal{G}_{[\mathfrak{b}]}[1]=[\mathfrak{b}], \quad \mathcal{G}_{[\mathfrak{a}]}[1]=[\mathfrak{a}]
$$

and natural transformations $i: \mathcal{G}_{[\mathfrak{a}]} \rightarrow \mathcal{G}_{[\mathfrak{b}]}, p: \mathcal{G}_{[\mathfrak{b}]} \rightarrow \mathcal{G}_{[\mathfrak{a}]}$ such that $p \circ i=\operatorname{id}_{\mathcal{G}_{[\mathfrak{a}]}}$.

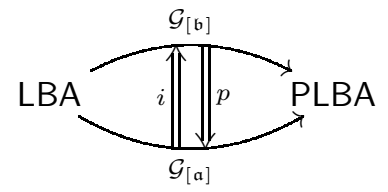

Moreover, PLBA satisfies the following universal property: for any symmetric tensor category $\mathcal{C}$ with the same property above as PLBA, there exists, up to a unique 
equivalence, a unique symmetric tensor functor PLBA $\rightarrow \mathcal{C}$ such that the following diagram commutes

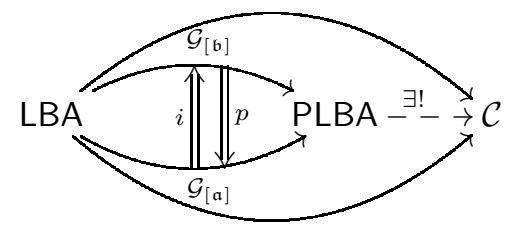

A module over PLBA in Vect $t_{k}$ is a split pair $(\mathfrak{b}, \mathfrak{a})$ of Lie bialgebras over $k$, and a morphism $(\mathfrak{b}, \mathfrak{a}) \rightarrow\left(\mathfrak{b}^{\prime}, \mathfrak{a}^{\prime}\right)$ between two such pairs is a pair of morphisms $(f, g) \in \operatorname{LBA}\left(\mathfrak{a}, \mathfrak{a}^{\prime}\right) \times \operatorname{LBA}\left(\mathfrak{b}, \mathfrak{b}^{\prime}\right)$ such that the following diagrams commute
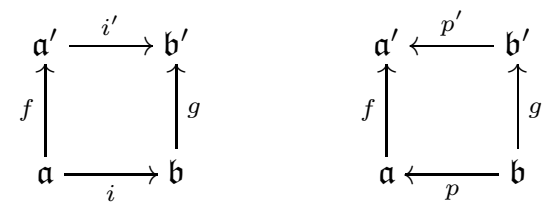

7.3. PROP description of the Verma module $L_{-}$. Let $\pi \in \operatorname{End}_{\mathrm{PLBA}}([\mathfrak{b}])$ be the idempotent $i \circ p$. The kernel of $\pi,[\mathfrak{m}]:=([\mathfrak{b}], 1-\pi)$, is an object in the Karoubi envelope of PLBA, which is a both a Lie ideal and coideal of $[\mathfrak{b}]$.

The module $L_{-}$introduced in $\S 3.7$ can be realised in PLBA. As an object, $\left[L_{-}\right]$ is equal to $S[\mathfrak{m}]$. The structure of Drinfeld-Yetter $[\mathfrak{b}]$-module is determined in the following way

- The action $\pi_{[\mathfrak{m}]}$ of $[\mathfrak{m}]$ is defined by the multiplication map

$$
\bigoplus_{0 \leq j \leq i+1} m_{1, i}^{j}:[\mathfrak{m}] \otimes S[\mathfrak{m}] \rightarrow S[\mathfrak{m}]
$$

given by Baker-Campbell-Hausdorff series.

- The action $\pi_{[\mathfrak{a}]}$ of $[\mathfrak{a}]$ is given by extending its action on $[\mathfrak{m}]$, which is an ideal in $[\mathfrak{b}]$, to $T[\mathfrak{m}]=\bigoplus_{n \geq 0}[\mathfrak{m}]^{\otimes n}$ and restricting it to $S[\mathfrak{m}]$.

- The action of $[\mathfrak{b}]$ is determined by those of $[\mathfrak{a}]$, $[\mathfrak{m}]$ since $[\mathfrak{b}]$ is the semidirect product $[\mathfrak{a}] \ltimes[\mathfrak{m}]$, and $\pi_{[\mathfrak{a}]}, \pi_{[\mathfrak{m}]}$ satisfy the following identity

$$
\pi_{[\mathfrak{a}]} \circ\left(\mathrm{id}_{[\mathfrak{a}]} \otimes \pi_{[\mathfrak{m}]}\right)-\pi_{[\mathfrak{m}]} \circ\left(\mathrm{id}_{[\mathfrak{m}]} \otimes \pi_{[\mathfrak{a}]}\right) \circ(12)=\pi_{[\mathfrak{m}]} \circ\left([,] \otimes \mathrm{id}_{V}\right)
$$

as morphisms $[\mathfrak{a}] \otimes[\mathfrak{m}] \otimes\left[L_{-}\right] \rightarrow\left[L_{-}\right]$.

- The coaction $\pi^{*}$ of $[\mathfrak{b}]$ on $\left[L_{-}\right]$is then uniquely determined by requiring that it be

- trivial on $S^{0}[\mathfrak{m}]$;

- compatible with the action of the ideal $[\mathfrak{m}]$ via the relation

$$
\pi^{*} \circ \pi_{[\mathfrak{m}]}=\mathrm{id} \otimes \pi_{[\mathfrak{m}]} \circ(12) \circ \mathrm{id} \otimes \pi^{*}-\mathrm{id} \otimes \pi_{[\mathfrak{m}]} \circ \delta \otimes \mathrm{id}+[,] \otimes \mathrm{id} \circ \mathrm{id} \otimes \pi^{*} .
$$

When $[\mathfrak{a}]=0$, the description of $\left[L_{-}\right]$reproduces that of the Verma module $\left[M_{-}\right]$ given in $\S 6.9$. More precisely, since $0 \hookrightarrow[\mathfrak{b}]$ is a split pair in $\underline{\mathrm{LBA}}$, there is a unique

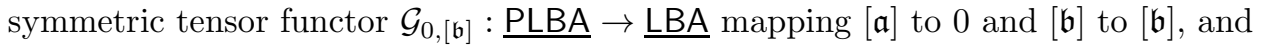
$\mathcal{G}_{0,[\mathfrak{b}]}\left[L_{-}\right]=\left[M_{-}\right]$as Drinfeld-Yetter modules over $[\mathfrak{b}]$. Moreover, the uniqueness of the coaction $\pi^{*}$ implies that if $(\mathfrak{a}, \mathfrak{b})$ is a split pair of Lie bialgebras, and $\mathcal{G}_{\mathfrak{a}, \mathfrak{b}}$ : LBA $\rightarrow$ Vect $_{\mathrm{k}}$ a corresponding realisation functor, $\mathcal{G}_{\mathfrak{a}, \mathfrak{b}}\left[L_{-}\right]$is the Drinfeld-Yetter module $L_{-}=\operatorname{Ind}_{\mathfrak{p}_{+}}^{\mathfrak{g}_{\mathfrak{b}}} \mathrm{k}$ introduced in $\S 3.7$. 
7.4. Matched pairs of Lie bialgebras. In order to describe the propic construction of the Verma module $N_{+}^{\vee}$, we shall need the following notion from [25, Section 8.3], which provides a generalisation of the notion of Drinfeld double.

Two Lie algebras $\left(\mathfrak{c},[,]_{\mathfrak{c}}\right)$ and $\left(\mathfrak{d},[,]_{\mathfrak{d}}\right)$ form a matched pair if there are maps

$$
\triangleright: \mathfrak{c} \otimes \mathfrak{d} \rightarrow \mathfrak{d} \quad \text { and } \quad \triangleleft: \mathfrak{c} \otimes \mathfrak{d} \rightarrow \mathfrak{c}
$$

such that

(i) $\triangleright$ is a left action of $\mathfrak{c}$ on $\mathfrak{d}$, i.e.,

$$
\triangleright \circ[,]_{\mathfrak{c}} \otimes \mathrm{id}=\triangleright \circ \mathrm{id} \otimes \triangleright \circ(\mathrm{id}-(12))
$$

and $\triangleleft$ is a right action of $\mathfrak{d}$ on $\mathfrak{c}$, i.e.,

$$
\triangleleft \circ \mathrm{id} \otimes[,]_{\mathrm{o}}=\triangleleft \circ \triangleleft \otimes \mathrm{id} \circ(\mathrm{id}-(23)) ;
$$

(ii) $\triangleleft, \triangleright$ satisfy the compatibility conditions

$$
\begin{aligned}
& \triangleleft \circ[,]_{\mathfrak{c}} \otimes \mathrm{id}=[,]_{\mathfrak{c}} \circ \triangleleft \otimes \mathrm{id} \circ(23)+[,]_{\mathfrak{c}} \circ \mathrm{id} \otimes \triangleleft+\triangleleft \circ \mathrm{id} \otimes \triangleright \circ(\mathrm{id}-(12)) \\
& \quad \text { and } \\
& \triangleright \circ \mathrm{id} \otimes[,]_{\mathfrak{o}}=[,]_{\mathfrak{o}} \circ \triangleright \otimes \mathrm{id}+[,]_{\mathfrak{o}} \circ \mathrm{id} \otimes \triangleright \circ(12)+\triangleright \circ \triangleleft \otimes \mathrm{id} \circ(\mathrm{id}-(23)) .
\end{aligned}
$$

The conditions (i)-(ii) are equivalent to the requirement that the vector space $\mathfrak{c} \oplus \mathfrak{d}$ is endowed with a Lie bracket for which $\mathfrak{c}, \mathfrak{d}$ are Lie subalgebras and, for $X \in \mathfrak{c}$ and $Y \in \mathfrak{d}$,

$$
[X, Y]=X \triangleright Y+X \triangleleft Y
$$

With the above bracket, $\mathfrak{c} \oplus \mathfrak{d}$ is denoted by $\mathfrak{c} \triangleright \triangleleft \mathfrak{d}$ and called the double cross sum Lie algebra of $\mathfrak{c}, \mathfrak{d}$.

EXAMPLE. If $\left(\mathfrak{a},[,]_{\mathfrak{a}}, \delta_{\mathfrak{a}}\right)$ is a Lie bialgebra, the Lie algebras $\left(\mathfrak{a},[,]_{\mathfrak{a}}\right)$ and $\left(\mathfrak{a}^{*}, \delta_{\mathfrak{a}}^{t}\right)$ form a matched pair with respect to the coadjoint action of $\mathfrak{a}$ on $\mathfrak{a}^{*}$ and the opposite coadjoint action of $\mathfrak{a}^{*}$ on $\mathfrak{a}$. The corresponding double cross sum Lie algebra $\mathfrak{a} \triangleright \triangleleft \mathfrak{a}^{*}$ is the Drinfeld double of $\mathfrak{a}$.

7.5. Extended PLBA. The definition of the Verma module $N_{+}^{\vee}$ relies on the parabolic subalgebra $\mathfrak{p}_{-}=\mathfrak{b}_{-} \oplus \mathfrak{a}_{+}=\mathfrak{m}_{-} \oplus \mathfrak{g}_{\mathfrak{a}} \subset \mathfrak{g}_{\mathfrak{b}}$, which cannot be realised in PLBA. We therefore introduce the colored PROP PLBA ${ }^{+}$obtained by adding to the presentation of PLBA a Lie algebra object $\left[\mathfrak{a}^{\sharp}\right]$ in matched pairing with the split pair $[\mathfrak{a}] \stackrel{i}{\longrightarrow}[\mathfrak{b}] \stackrel{p}{\longrightarrow}[\mathfrak{a}]$. Namely, we assume that $[\mathfrak{b}]$ and $\left[\mathfrak{a}^{\sharp}\right]$ (and, respectively, [a $]$ and $\left.\left[\mathfrak{a}^{\sharp}\right]\right)$ are endowed with mutual left and right actions

$$
\begin{aligned}
& \triangleright_{[\mathfrak{b}]}:[\mathfrak{b}] \otimes\left[\mathfrak{a}^{\sharp}\right] \rightarrow\left[\mathfrak{a}^{\sharp}\right] \quad \text { and } \quad \triangleleft_{[\mathfrak{b}]}:[\mathfrak{b}] \otimes\left[\mathfrak{a}^{\sharp}\right] \rightarrow[\mathfrak{b}], \\
& \triangleright_{[\mathfrak{a}]}:[\mathfrak{a}] \otimes\left[\mathfrak{a}^{\sharp}\right] \rightarrow\left[\mathfrak{a}^{\sharp}\right] \quad \text { and } \quad \triangleleft_{[\mathfrak{a}]}:[\mathfrak{a}] \otimes\left[\mathfrak{a}^{\sharp}\right] \rightarrow[\mathfrak{a}],
\end{aligned}
$$

satisfying the compatibility conditions of $\S 7.4$. Further, we assume that $i, p$ are morphisms of matched pairs, i.e.,

$$
\triangleright_{[\mathfrak{b}]} \circ i \otimes \mathrm{id}=\triangleright_{[\mathfrak{a}]} \quad \text { and } \quad \triangleright_{[\mathfrak{b}]}=\triangleright_{[\mathfrak{a}]} \circ p \otimes \mathrm{id}
$$

as maps $[\mathfrak{a}] \otimes\left[\mathfrak{a}^{\sharp}\right] \rightarrow\left[\mathfrak{a}^{\sharp}\right]$ and $[\mathfrak{b}] \otimes\left[\mathfrak{a}^{\sharp}\right] \rightarrow[\mathfrak{a}]$ respectively (similarly for $\triangleleft_{[\mathfrak{b}]}, \triangleleft_{[\mathfrak{a}]}$ ). It follows that $\left([\mathfrak{a}] \bowtie \triangleleft\left[\mathfrak{a}^{\sharp}\right],[\mathfrak{b}] \bowtie \triangleleft\left[\mathfrak{a}^{\sharp}\right]\right)$ is a split pair of Lie algebras. Additionally, we 
assume there is a morphism $r_{[\mathfrak{a}]}:[0] \rightarrow[\mathfrak{a}] \otimes\left[\mathfrak{a}^{\sharp}\right]$ satisfying the classical Yang-Baxter equation, together with the relations

$$
\begin{aligned}
\delta_{[\mathfrak{a}]} \otimes \operatorname{id}_{\left[\mathfrak{a}^{\sharp}\right]} \circ r_{[\mathfrak{a}]} & =\left[r_{[\mathfrak{a}]_{13}}, r_{\left.[\mathfrak{a}]_{23}\right]},\right. \\
\operatorname{id} \otimes[,]_{\left[\mathfrak{a}^{\sharp}\right]} \circ r_{[\mathfrak{a}]} \otimes \mathrm{id} & =\triangleleft_{[\mathfrak{a}]} \otimes \mathrm{id} \circ \mathrm{id} \otimes r_{[\mathfrak{a}]}, \\
{[,]_{[\mathfrak{a}]} \otimes \mathrm{id} \circ \mathrm{id} \otimes r_{[\mathfrak{a}]} } & =\operatorname{id~} \otimes \triangleright_{[\mathfrak{a}]} \circ r_{[\mathfrak{a}]} \otimes \mathrm{id},
\end{aligned}
$$

as morphisms $[0] \rightarrow[\mathfrak{a}] \otimes[\mathfrak{a}] \otimes\left[\mathfrak{a}^{\sharp}\right],\left[\mathfrak{a}^{\sharp}\right] \rightarrow[\mathfrak{a}] \otimes\left[\mathfrak{a}^{\sharp}\right]$, and $[\mathfrak{a}] \rightarrow[\mathfrak{a}] \otimes\left[\mathfrak{a}^{\sharp}\right]$, respectively.

In particular, equation (7.1) encodes the fact that the copairing $r_{[\mathfrak{a}]}$ between $[\mathfrak{a}]$ and $\left[\mathfrak{a}^{*}\right]$ identifies the transpose of $\delta_{[\mathfrak{a}]}$ with the bracket on $\left[\mathfrak{a}^{\sharp}\right]$. ${ }^{10}$

Let $\left(\mathfrak{b}, \mathfrak{a}, \mathfrak{a}^{\sharp}\right),\left(\mathfrak{b}^{\prime}, \mathfrak{a}^{\prime},\left(\mathfrak{a}^{\sharp}\right)^{\prime}\right)$ be two modules over PLBA ${ }^{+}$. Then a morphism of $\operatorname{PLBA}^{+}$-modules $\left(\mathfrak{b}, \mathfrak{a}, \mathfrak{a}^{\sharp}\right) \rightarrow\left(\mathfrak{b}^{\prime}, \mathfrak{a}^{\prime},\left(\mathfrak{a}^{\sharp}\right)^{\prime}\right)$ is a triple $(h, g, f)$ where $(h, g):(\mathfrak{b}, \mathfrak{a}) \rightarrow$ $\left(\mathfrak{b}^{\prime}, \mathfrak{a}^{\prime}\right)$ is a morphism of split pairs, $(g, f):\left(\mathfrak{a},\left(\mathfrak{a}^{\sharp}\right)\right) \rightarrow\left(\mathfrak{a}^{\prime},\left(\mathfrak{a}^{\sharp}\right)^{\prime}\right)$ and $(h, f):$ $\left(\mathfrak{b}, \mathfrak{a}^{\sharp}\right) \rightarrow\left(\mathfrak{b}^{\prime},\left(\mathfrak{a}^{\sharp}\right)^{\prime}\right)$ are morphisms of matched pairs, and $(h \otimes g)\left(r_{\mathfrak{a}}\right)=r_{\mathfrak{a}^{\prime}}$.

We shall call a module $\left(\mathfrak{b}, \mathfrak{a}, \mathfrak{a}^{\sharp}\right)$ over PLBA ${ }^{+}$in Vect ${ }_{k}$ an enhanced split pair if $\mathfrak{a}^{\sharp}=\mathfrak{a}^{*}$ and $r_{\mathfrak{a}}$ is the $r$-matrix of $\mathfrak{a}$.

7.6. PROP description of the Verma module $N_{+}^{\vee}$. The module $\left[N_{+}^{\vee}\right]$ can be realised on the object $\widehat{S[\mathfrak{p}}],[\mathfrak{p}]=[\mathfrak{b}] \oplus\left[\mathfrak{a}^{\sharp}\right]$, formally added to $\underline{\mathrm{PLBA}^{+}}$. In describing the structure of the Drinfeld-Yetter module on $\left[N_{+}^{\vee}\right]$, we proceed as in $\S 6.12$. This is obtained as the propic solution of the universal property

$$
\operatorname{Hom}_{\mathfrak{b}}^{\mathfrak{b}}\left(V, \mathcal{G}\left[N_{+}^{\vee}\right]\right) \underset{\phi}{\stackrel{\psi}{\rightleftarrows}} \operatorname{Hom}_{\mathfrak{m}}(V, \mathbf{k})
$$

where $\psi$ denotes the composition with the projection $\varepsilon_{\left[N_{+}^{\vee}\right]}:\left[N_{+}^{\vee}\right] \rightarrow[0]$ and $\phi$ is the map

$$
\phi=\mathrm{id} \otimes-\circ \sum_{n, m \geq 0} \operatorname{Sym}_{n} \otimes \operatorname{Sym}_{m} \otimes \mathrm{id} \circ \mathrm{id}^{\otimes m} \otimes \pi_{V}^{(m)} \circ \mathrm{id}^{\otimes n} \otimes r_{\mathfrak{a}}^{(m)} \circ\left(\pi_{V}^{*}\right)^{(n)} .
$$

More precisely, $\left[N_{+}^{\vee}\right]$ is constructed on the object $\widehat{S}[\mathfrak{p}]=\widehat{S}[\mathfrak{b}] \otimes \widehat{S}\left[\mathfrak{a}^{\sharp}\right]$ with the following Drinfeld-Yetter structure.

- As in $\S 6.12$, the coaction $\pi_{\left[N_{+}^{\vee}\right]}^{*}$ is obtained from that of $\left[M_{+}^{\vee}\right]$, i.e., $\pi_{\left[N_{+}^{\vee}\right]}^{*}=$ $\pi_{\left[M_{+}^{\vee}\right]}^{*} \otimes \mathrm{id}_{\widehat{S}\left[\mathfrak{a}^{\sharp}\right]}$. In particular, the projection from $\left[N_{+}^{\vee}\right]$ to $\left[M_{+}^{\vee}\right]$ is a map of $[\mathfrak{b}]$-comodules.

- The formula for the action $\pi_{\left[N_{+}^{\vee}\right]}$ is obtained by imposing the following two conditions:

$$
\varepsilon_{\left[N_{+}^{\vee}\right]} \circ \pi_{\left[N_{+}^{\vee}\right]}=\varepsilon_{\left[N_{+}^{\vee}\right]} \circ \pi_{\left[N_{+}^{\vee}\right]} \circ p \otimes \mathrm{id}
$$

as maps from $[\mathfrak{b}] \otimes\left[N_{+}^{\vee}\right] \rightarrow[0]$, and

$$
\phi\left(\varepsilon_{\left[N_{+}^{\vee}\right]}\right)=\operatorname{id}_{\left[N_{+}^{\vee}\right]}
$$

as maps from $\left[N_{+}^{\vee}\right]$ to $\widehat{S}[\mathfrak{b}] \otimes \widehat{S}\left[\mathfrak{a}^{\sharp}\right]=\left[N_{+}^{\vee}\right]$. One sees easily that the action $\pi_{\left[N_{+}^{\vee}\right]}^{*}$ exists and it is uniquely defined by these properties. Namely, one

\footnotetext{
${ }^{10}$ The PROP PLBA ${ }^{+}$is a generalisation to the relative case of the Drinfeld double PROP $D_{\oplus}($ LBA $)$ introduced in [12, Section 3].
} 
has

$$
\begin{aligned}
\pi_{\left[N_{+}^{\vee}\right]}= & \operatorname{id}_{\left[N_{+}^{\vee}\right]} \circ \pi_{\left[N_{+}^{\vee}\right]} \\
= & \left(\sum_{n, m \geq 0} \operatorname{id}_{S^{n}[\mathfrak{b}] \otimes S^{m}\left[\mathfrak{a}^{\sharp}\right]} \otimes \varepsilon \circ \operatorname{Sym}_{n} \otimes \operatorname{Sym}_{m} \otimes \mathrm{id} \circ\right. \\
& \left.\circ \mathrm{id}^{\otimes m} \otimes \pi_{\left[N_{+}^{\vee}\right]}^{(m)} \circ \mathrm{id}^{\otimes n} \otimes r_{\mathfrak{a}}^{(m)} \circ\left(\pi_{\left[N_{+}^{\vee}\right]}^{*}\right)^{(n)}\right) \circ \pi_{\left[N_{+}^{\vee}\right]} .
\end{aligned}
$$

By iterated application of the compatibility condition $(2.4)$ on $\left(\pi_{\left[N_{+}^{\vee}\right]}^{*}\right)_{\left[N_{+}\right.}^{(n)}$ 。 $\pi_{\left[N_{+}^{\vee}\right]}$, relations (2.2) and (7.3), one obtains an explicit description of $\pi_{\left[N_{+}^{\vee}\right]}$, given exclusively in terms of morphisms in $\mathrm{PLBA}^{+}$.

- The description of the Drinfeld-Yetter structure over $[\mathfrak{a}]^{\text {op }}$ is obtained with a similar argument.

When $[\mathfrak{a}]=0=\left[\mathfrak{a}^{\sharp}\right]$, the description of $\left[N_{+}^{\vee}\right]$ reproduces that of the Verma modules $\left[M_{+}^{\vee}\right]$ given in $\S 6.12$. More precisely, since $([\mathfrak{b}], 0,0)$ is an enhanced split pair in LBA, there is a unique symmetric tensor functor $\mathcal{G}_{([\mathfrak{b}], 0,0)}: \mathrm{PLBA}^{+} \rightarrow$ LBA mapping $[\mathfrak{a}],\left[\mathfrak{a}^{\sharp}\right]$ to $0,[\mathfrak{b}]$ to $[\mathfrak{b}]$, and $\mathcal{G}\left[N_{+}^{\vee}\right]=\left[M_{+}^{\vee}\right]$ as Drinfeld-Yetter $[\mathfrak{b}]$-modules. Moreover, the uniqueness of the action $\pi_{N_{+}^{\vee}}$ implies that if $(\mathfrak{b}, \mathfrak{a})$ is a split pair of Lie bialgebras and $\mathcal{G}_{\left(\mathfrak{b}, \mathfrak{a}, \mathfrak{a}^{*}\right)}: \mathrm{PLBA}^{+} \rightarrow$ Vect $_{\mathrm{k}}$ a corresponding realisation functor, $\mathcal{G}_{\left(\mathfrak{b}, \mathfrak{a}, \mathfrak{a}^{*}\right)}\left[N_{+}^{\vee}\right]$ is the Drinfeld-Yetter $\left(\mathfrak{b}, \mathfrak{a}^{\text {op }}\right)$-module $N_{+}^{\vee}$ introduced in $\S 3.7$

7.7. The propic construction of the twist. Let $D Y_{[\mathfrak{a}]}^{\Phi}$ and $D Y_{[\mathfrak{b}]}^{\Phi}$ be the categories of deformed Drinfeld-Yetter modules in the symmetric monoidal category $\underline{\mathrm{PLBA}}^{+} \llbracket \hbar \rrbracket$ over the Lie bialgebras $[\mathfrak{a}]$ and $[\mathfrak{b}]$ respectively. By Frobenius reciprocity, for every $V, W \in \mathrm{DY}_{[\mathfrak{b}]}^{\Phi}$, we get an isomorphism

$$
\operatorname{Hom}_{[\mathfrak{b}]}^{[\mathfrak{b}]}\left(\left[L_{-}\right] \otimes V^{0},\left[N_{+}^{\vee}\right] \otimes W\right) \simeq \operatorname{Hom}_{\underline{P L B A}^{+}}(V, W) .
$$

Let $\psi_{V}^{[\mathfrak{a}],[\mathfrak{b}]}:\left[L_{-}\right] \otimes V^{0} \rightarrow\left[N_{+}^{\vee}\right] \otimes V$ be the map corresponding to the identity on $V$ and set $\eta_{V}^{[\mathfrak{a}],[\mathfrak{b}]}=\psi_{V}^{[\mathfrak{a}],[\mathfrak{b}]} \circ 1_{-} \otimes \mathrm{id}_{V}$, where $1_{-}$denotes the inclusion of $[0]$ in $\left[L_{-}\right]$.

We define a map $J_{[\mathfrak{a}],[\mathfrak{b}]}: V \otimes W \rightarrow V \otimes W$ by

$$
J_{[\mathfrak{a}],[\mathfrak{b}]}=1_{+}^{\otimes 2} \otimes \mathrm{id}^{\otimes 2} \circ A_{\Phi} \circ \eta_{V}^{[\mathfrak{a}],[\mathfrak{b}]} \otimes \eta_{W}^{[\mathfrak{a}],[\mathfrak{b}]},
$$

where $1_{+}$is the projection from $\left[N_{+}^{\vee}\right]$ to $[0]$, and $A_{\Phi}$ is as in $\S 3.17$.

\section{Proposition.}

(i) The map $J_{[\mathfrak{a}],[\mathfrak{b}]}$ defines a tensor structure on the propic restriction functor $\operatorname{Res}_{[\mathfrak{a}],[\mathfrak{b}]}: \mathrm{DY}_{[\mathfrak{b}]}^{\Phi} \rightarrow \mathrm{DY}_{[\mathfrak{a}]}^{\Phi}$ given by

$$
\operatorname{Res}_{[\mathfrak{a}],[\mathfrak{b}]}\left(V, \pi, \pi^{*}\right)=\left(V, \pi \circ i \otimes \operatorname{id}_{V}, p \otimes \operatorname{id}_{V} \circ \pi^{*}\right)
$$

(ii) Let $\left(\mathfrak{b}, \mathfrak{a}, \mathfrak{a}^{*}\right)$ be an enhanced split pair with a realisation functor $\mathcal{G}=$ $\mathcal{G}_{\left(\mathfrak{b}, \mathfrak{a}, \mathfrak{a}^{*}\right)}: \mathrm{PLBA}^{+} \rightarrow$ Vect $_{\mathfrak{k}}$. Then the tensor structure $\mathcal{G}\left(J_{[\mathfrak{a}],[\mathfrak{b}]}\right)$ on the restriction functor $\operatorname{Res}_{\mathfrak{a}, \mathfrak{b}}: \mathrm{DY}_{\mathfrak{b}}^{\Phi} \rightarrow \mathrm{DY}_{\mathfrak{a}}^{\Phi}$ coincides, under the identification $\operatorname{Res}_{\mathfrak{a}, \mathfrak{b}} \simeq F_{\mathfrak{a}, \mathfrak{b}}$, with the twist $J_{\mathfrak{a}, \mathfrak{b}}$ constructed in Section $\S 3$. 
Proof. (i) follows from a straighforward adaptation of $\S 3.18-\S 3.21$. (ii) follows by direct inspection.

It follows that the twist $J_{\mathfrak{a}, \mathfrak{b}}$ is functorial with respect to morphisms of enhanced split pairs. Namely, we have the following

Corollary. Let $\left[V_{i}\right] \in \mathrm{DY}_{[\mathfrak{[}]}^{\Phi}, i=1,2,\left(\mathfrak{b}, \mathfrak{a}, \mathfrak{a}^{*}\right)$ and $\left(\mathfrak{b}^{\prime}, \mathfrak{a}^{\prime},\left(\mathfrak{a}^{*}\right)^{\prime}\right)$ two enhanced split pairs with realisation functors $\mathcal{G}, \mathcal{G}^{\prime}: \mathrm{PLBA}^{+} \rightarrow$ Vect $_{\mathrm{k}}$, and set $V_{i}=\mathcal{G}\left[V_{i}\right], V_{i}^{\prime}=$ $\mathcal{G}^{\prime}\left[V_{i}\right]$. Then for any $\rho \in \operatorname{Fun}^{\otimes}\left(\mathcal{G}, \mathcal{G}^{\prime}\right)$ (or equivalently, for any morphism $(f, g, h)$ : $\left.\left(\mathfrak{b}, \mathfrak{a}, \mathfrak{a}^{*}\right) \stackrel{(f, g, h)}{\longrightarrow}\left(\mathfrak{b}^{\prime}, \mathfrak{a}^{\prime},\left(\mathfrak{a}^{\prime}\right)^{*}\right)\right)$, the following diagram is commutative

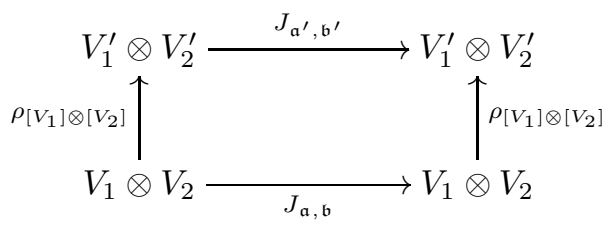

7.8. Quantisation of propic modules in $\mathrm{PLBA}^{+}$. The quantisation formulae given in $\S 6.14-\S 6.16$ have their analogues in $\mathrm{PLBA}^{+}$. Specifically, since the modules $M_{-}^{[\mathfrak{b}]}, M_{+}^{\vee[\mathfrak{b}]}$ and $M_{-}^{[\mathfrak{a}]}, M_{+}^{\vee[\mathfrak{a}]}$ have their own realisation in $\underline{\mathrm{PLBA}^{+}}$, there are isomorphisms

$$
\begin{aligned}
& \operatorname{Hom}_{[\mathfrak{[ b ]}}^{[\mathfrak{b}]}\left(M_{-}^{[\mathfrak{b}]} \otimes V^{0}, M_{+}^{\vee[\mathfrak{b}]} \otimes W\right) \simeq \operatorname{Hom}_{\underline{{ }_{\text {LBB }}^{+}}}(V, W), \\
& \operatorname{Hom}_{[\mathfrak{[ a ]}}^{[\mathfrak{a}]}\left(M_{-}^{[\mathfrak{a}]} \otimes V^{0}, M_{+}^{\vee[\mathfrak{a}]} \otimes W\right) \simeq \operatorname{Hom}_{\underline{P L B A}^{+}}(V, W),
\end{aligned}
$$

for every Drinfeld-Yetter [ $\mathfrak{b}]$-modules $V, W$ in $\underline{\mathrm{PLBA}}^{+}$, with analogous distinguished morphisms $\psi_{V}: M_{-}^{[\mathfrak{b}]} \otimes V \rightarrow M_{+}^{\vee[\mathfrak{b}]} \otimes V$ and $\eta_{V}=\psi_{V} \circ 1_{-} \otimes$ id $_{V}$, and similarly for $M_{-}^{[\mathfrak{a}]}, M_{+}^{\vee[\mathfrak{a}]}$. These allow to define an action of $Q[B]=M_{-}^{[\mathfrak{b}]}$ and $Q[A]=M_{-}^{[\mathfrak{a}]}$ on $V$

$$
\rho_{V}=1_{+} \otimes 1_{+} \otimes \text { id } \circ \text { id } \otimes \psi_{V} \circ \Phi \circ \eta_{M} \otimes \text { id }
$$

and a coaction

$$
\rho_{V}^{*}=R^{J} \circ \widetilde{\iota} \otimes \mathrm{id}_{V}
$$

(similarly for $[\mathfrak{a}]$ ) providing a propic version $\widetilde{\mathfrak{f}}_{[\mathfrak{b}]}, \widetilde{\mathrm{f}}_{[\mathfrak{a}]}$ of the equivalences $\widetilde{F}_{\mathfrak{b}}, \widetilde{F}_{\mathfrak{a}}$ over the symmetric category $\mathrm{PLBA}^{+} \llbracket \hbar \rrbracket$. In particular, the quantisation of DrinfeldYetter $[\mathfrak{b}]$-modules in $\mathrm{PLBA}^{+} \llbracket \hbar \rrbracket$ is functorial with respect to morphisms of enhanced split pairs. Namely we have the following

Corollary. Let $\left([V], \pi, \pi^{*}\right)$ be a Drinfeld-Yetter $[\mathfrak{b}]-$ module in PLBA (resp. PLBA ${ }^{+}$), $(\mathfrak{b}, \mathfrak{a})$ and $\left(\mathfrak{b}^{\prime}, \mathfrak{a}^{\prime}\right)$ two split pairs with realisation functors $\mathcal{G}, \mathcal{G}^{\prime}:$ PLBA $\rightarrow$ Vect $_{k}$ (resp. $\left(\mathfrak{b}, \mathfrak{a}, \mathfrak{a}^{*}\right)$ and $\left(\mathfrak{b}^{\prime}, \mathfrak{a}^{\prime},\left(\mathfrak{a}^{*}\right)^{\prime}\right)$ two enhanced split pairs with realisation functors $\mathcal{G}, \mathcal{G}^{\prime}: \mathrm{PLBA}^{+} \rightarrow$ Vect $\left._{\mathrm{k}}\right)$, and set

$$
\left(V, \pi_{V}, \pi_{V}^{*}\right)=\mathcal{G}\left([V], \pi, \pi^{*}\right) \in \mathrm{DY}_{\mathfrak{b}} \quad \text { and } \quad\left(V^{\prime}, \pi_{V^{\prime}}, \pi_{V^{\prime}}^{*}\right)=\mathcal{G}^{\prime}\left([V], \pi, \pi^{*}\right) \in \mathrm{DY}_{\mathfrak{b}^{\prime}} .
$$

Then, for any $\rho \in \operatorname{Fun}^{\otimes}\left(\mathcal{G}, \mathcal{G}^{\prime}\right)$ (or equivalently, for any morphism $(f, g):(\mathfrak{b}, \mathfrak{a}) \rightarrow$ $\left(\mathfrak{b}^{\prime}, \mathfrak{a}^{\prime}\right)\left(\right.$ resp. $\left.\left.(f, g, h):\left(\mathfrak{b}, \mathfrak{a}, \mathfrak{a}^{*}\right) \stackrel{(f, g, h)}{\longrightarrow}\left(\mathfrak{b}^{\prime}, \mathfrak{a}^{\prime},\left(\mathfrak{a}^{\prime}\right)^{*}\right)\right)\right)$, the following diagrams are 
commutative

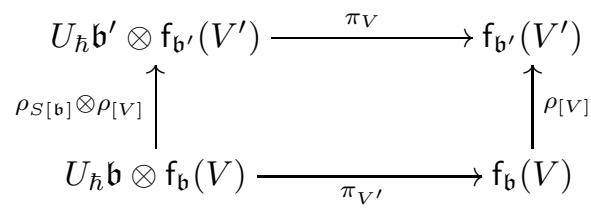

and

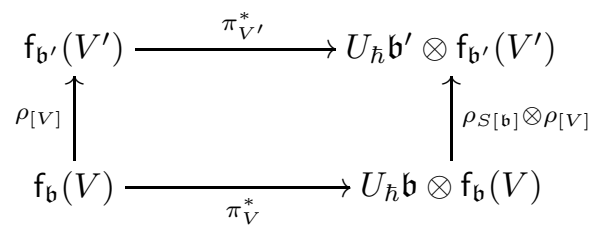

7.9. The isomorphism $\widetilde{F}_{\mathfrak{b}}\left(L_{-}\right) \simeq L_{-}^{\hbar}$. We now prove part (i) of Theorem 4.13. Part (ii) is proved in $\S 7.10$.

By Lemma 2.23 and Proposition 4.11, the semiclassical limits of $\widetilde{F}_{\mathfrak{b}}\left(L_{-}\right)$and $L_{-}^{\hbar}$ are both equal to $L_{-}$. It therefore suffices to construct an intertwiner $L_{-}^{\hbar} \rightarrow \widetilde{F}_{\mathfrak{b}}\left(L_{-}\right)$ in $\mathrm{DY}_{U_{\hbar} \mathfrak{b}}$ whose reduction mod $\hbar$ is the identity. By the universal property of $L_{-}^{\hbar}(4.17)$, this amounts to constructing a linear map $\ell_{\hbar}: \mathrm{k} \llbracket \hbar \rrbracket \rightarrow \widetilde{F}_{\mathfrak{b}}\left(L_{-}\right)$which intertwines the action of $U_{\hbar} \mathfrak{a}$ and the coaction of $U_{\hbar} \mathfrak{b}$, and whose reduction $\bmod \hbar$ is the inclusion $\ell$ of the generating vector $1_{-} \in L_{-}$.

To this end, it is instructive to note that the intertwining properties of $\ell$ correspond to the commutativity of the diagrams
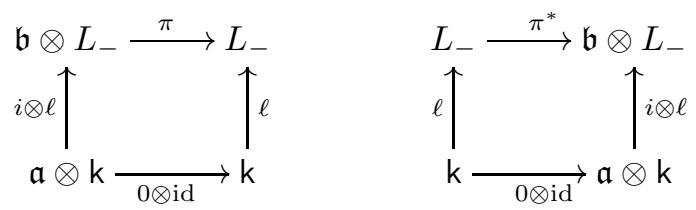

Moreover, if the trivial module $k \in D Y_{\mathfrak{a}}$ is thought of as the Verma module $L_{-}$ corresponding to the split pair $\mathfrak{a} \hookrightarrow \mathfrak{a}$, these diagrams arise from the functoriality of $L_{-}$with respect to the morphism of split pairs $(i, \mathrm{id}):(\mathfrak{a}, \mathfrak{a}) \rightarrow(\mathfrak{b}, \mathfrak{a})$.

Similarly, regarding $\mathrm{k} \llbracket \hbar \rrbracket$ as the trivial Drinfeld-Yetter module over $U_{\hbar} \mathfrak{a}$, the required interwining properties of the map $\ell_{\hbar}$ correspond to the diagrams
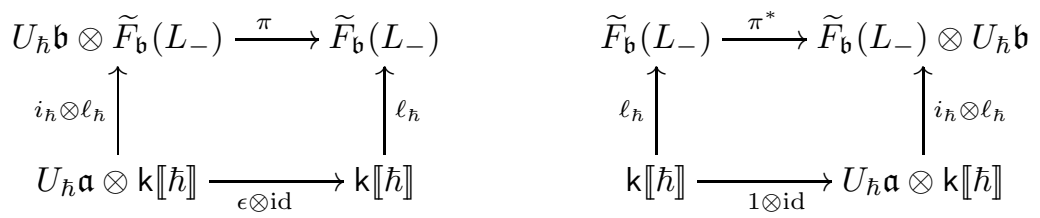

Since $\mathrm{DY}_{U_{\hbar} \mathfrak{a}} \ni \mathrm{k} \llbracket \hbar \rrbracket$ is equal to $\widetilde{F}_{\mathfrak{a}}(\mathrm{k} \llbracket \hbar \rrbracket)$, the existence of $\ell_{\hbar}$, the fact that it reduces to $\ell \bmod \hbar$, and the commutativity of the above diagrams follow from the functoriality of the Tannakian lift of $L_{-}$with respect to the morphism of split pairs $(i, \mathrm{id}):(\mathfrak{a}, \mathfrak{a}) \rightarrow(\mathfrak{b}, \mathfrak{a})$ (Corollary 7.8$)$. Moreover, $\ell_{\hbar}$ is a morphism of coalgebras since it maps the group-like generating vector of $L_{-}^{\hbar}$ to a group-like element of $\widetilde{F}_{\mathfrak{b}}\left(L_{-}\right)$. 
7.10. The isomorphism $\widetilde{F}_{\mathfrak{b}} \circ \widetilde{F}_{\mathfrak{a}}\left(N_{+}^{\vee}\right) \simeq\left(N_{+}^{\vee}\right)^{\hbar}$. We adopt the same strategy of $\S 7.9$ to prove part (ii) of Theorem 4.13.

By Proposition 4.11, the semiclassical limits of $\left(N_{+}^{\vee}\right)^{\hbar}$ is equal to $N_{+}^{\vee}$ as DrinfeldYetter $(\mathfrak{b}, \mathfrak{a})$-bimodules. Similarly, combining Lemma 2.23 and Proposition 2.25, one concludes that the semiclassical limit of $\widetilde{F}_{\mathfrak{b}} \circ \widetilde{F}_{\mathfrak{a}}\left(N_{+}^{\vee}\right) \simeq \widetilde{F}_{\mathfrak{b} \oplus \mathfrak{a}^{\text {op }}}\left(N_{+}^{\vee}\right)$ is equal to $N_{+}^{\vee}$ as Drinfeld-Yetter $(\mathfrak{b}, \mathfrak{a})$-bimodules. It therefore suffices to construct an intertwiner $\widetilde{F}_{\mathfrak{b}} \circ \widetilde{F}_{\mathfrak{a}}\left(N_{+}^{\vee}\right) \rightarrow\left(N_{+}^{\vee}\right)^{\hbar}$ in $\mathrm{DY}_{U_{\hbar} \mathfrak{b}}$ whose reduction mod $\hbar$ is the identity. By the universal property of $\left(N_{+}^{\vee}\right)^{\hbar}(4.19)$, this amounts to constructing a linear $\operatorname{map} n_{\hbar}: \widetilde{F}_{\mathfrak{b}} \circ \widetilde{F}_{\mathfrak{a}}\left(N_{+}^{\vee}\right) \rightarrow p_{\hbar}^{*}\left(N_{+}^{\vee}\right)_{\mathfrak{a}}^{\hbar}$ which intertwines the action of $U_{\hbar} \mathfrak{b}$ and coaction of $U_{\hbar} \mathfrak{a}$, and whose reduction mod $\hbar$ is the projection $n$ of $N_{+}^{\vee}$ onto $\left(N_{+}^{\vee}\right)_{\mathfrak{a}}$ (induced by the projection of (topological) Lie bialgebras $\mathfrak{p}=\mathfrak{b} \oplus \mathfrak{a}^{*} \rightarrow \mathfrak{a} \oplus \mathfrak{a}^{*}$ ).

We observe as in $\S 7.9$ that the intertwining properties of $n$ corresponds to the commutativity of the diagrams
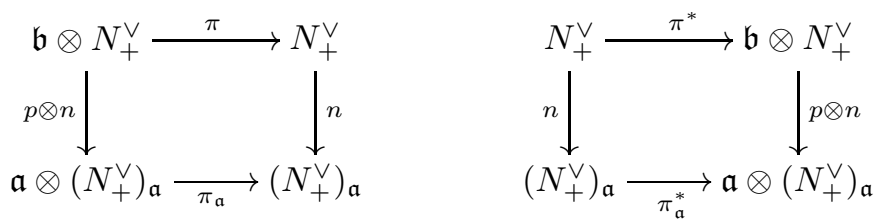

and analogue diagrams for the right action and coaction of $\mathfrak{a}$.

If the module $\left(N_{+}^{\vee}\right)_{\mathfrak{a}} \in \mathrm{DY}_{\mathfrak{a}}$ is thought of as the Verma module $N_{+}^{\vee}$ corresponding to the split pair $\mathfrak{a} \hookrightarrow \mathfrak{a}$, there diagrams arise from the functoriality of $N_{+}^{\vee}$ with respect to the morphism of enhanced split pairs $(p$, id, id $):\left(\mathfrak{b}, \mathfrak{a}, \mathfrak{a}^{*}\right) \rightarrow\left(\mathfrak{a}, \mathfrak{a}, \mathfrak{a}^{*}\right)$.

The required intertwining properties of $n_{\hbar}$ correspond to the commutativity of the diagrams
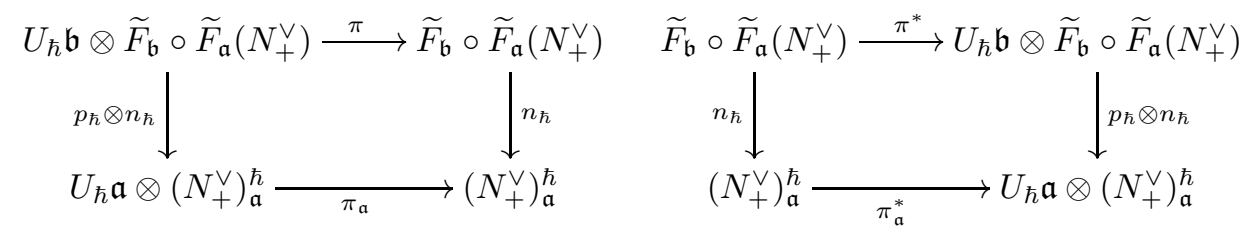

and their analogues for the right action and coaction of $U_{\hbar} \mathfrak{a}$. The existence of $n_{\hbar}$, the fact that it reduces to $n \bmod \hbar$, and the commutativity of the above diagrams follows from the functoriality of the Tannakian lift of $N_{+}^{\vee}$ with respect to the morphism of enhanced split pairs $(p$, id, id $):\left(\mathfrak{b}, \mathfrak{a}, \mathfrak{a}^{*}\right) \rightarrow\left(\mathfrak{a}, \mathfrak{a}, \mathfrak{a}^{*}\right)$. Finally, $n_{\hbar}$ is a morphism of algebras, since the algebra structure is uniquely determined by the universal property.

7.11. Propicity. The results from Sections $\S 4$ and $\S 5$ have analogous counterparts in the category $\mathrm{PLBA}^{+}$, since they rely exclusively on the propic realisations of the relative Verma modules $L_{-}, N_{+}^{\vee}$ in $\underline{\mathrm{PLBA}}^{+}$. In particular, we obtain a propic version of Theorem 5.2.1.

\section{Theorem.}

(i) The object $\left[L_{-}\right]$has a natural structure of Hopf algebra object in $\mathrm{DY}_{[\mathfrak{a}]}^{\Phi}$ with product

$$
m_{[L]}=1_{+} \otimes 1_{+} \otimes \mathrm{id} \circ \mathrm{id} \otimes \phi_{[L]} \circ \Phi \circ \eta_{[L]} \otimes \mathrm{id}
$$


and coproduct

$$
\Delta_{[L]}=J_{[\mathfrak{a}],[\mathfrak{b}]}^{-1} \circ \Delta_{0}
$$

where $\Delta_{0}$ is the standard coproduct on $\left[L_{-}\right]=S[\mathfrak{m}]$ and $J_{\mathfrak{a}, \mathfrak{b}}$ is the twist constructed in $\S 7 . \%$.

(ii) The quantised module $\widetilde{\mathrm{f}}_{[\mathrm{a}]}\left[L_{-}\right]$is a Hopf algebra object in $\mathrm{DY} \mathrm{Q}_{Q[A]}$ and the Radford biproduct $U_{\hbar}^{\mathrm{rel}}[\mathfrak{b}]=\widetilde{\mathrm{f}}_{\mathfrak{a}}\left[L_{-}\right] \star Q[A]$ is a quantisation of the Lie bialgebra $[\mathfrak{b}]$.

(iii) $U_{\hbar}^{\text {rel }}[\mathfrak{b}]$ splits over $U_{\hbar}[\mathfrak{a}]$ and there is an isomorphism of split pairs of Hopf

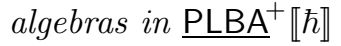

$$
u_{[\mathfrak{a}],[\mathfrak{b}]}:\left(U_{\hbar}[\mathfrak{b}], U_{\hbar}[\mathfrak{a}]\right) \rightarrow\left(U_{\hbar}^{\mathrm{rel}}[\mathfrak{b}], U_{\hbar}[\mathfrak{a}]\right) .
$$

The proof follows the same steps of Section $\S 5$ and it is therefore omitted.

\section{Alternative constructions}

In this section we discuss the relation between our construction and the quantisation functor described by P. Ševera in [27], whose construction and general principle we briefly review in $\S 8.1$.

In $\S 8.2$, we rephrase the case of a coisotropic subalgebra [27, Example 3] in the language of Section $\S 3$, and we show that this allows to construct a relative twist in the PROP PLBA $\hbar \rrbracket$. From the identification of the two constructions, it follows that the universal twist $J_{\mathfrak{a}, \mathfrak{b}}$, constructed in Sections $\S 3$ and $\S 7$ in the PROP $\underline{\mathrm{PLBA}}^{+} \llbracket \hbar \rrbracket$, is gauge equivalent to a universal twist in PLBA $\llbracket \hbar \rrbracket$, a result which is needed in [3].

8.1. Quantisation of Lie bialgebras revisited. In [27], Ševera provides an alternative construction of a universal quantisation functor, based on the following observations.

8.1.1. Let $\left(\mathcal{C}, \otimes_{\mathcal{C}}, \mathbf{1}_{\mathcal{C}}, \Phi_{\mathcal{C}}, \beta_{\mathcal{C}}\right)$ be a braided monoidal category. For any cocommutative coalgebra object $\left(M, \Delta_{M}, \epsilon_{M}\right) \in \operatorname{CoCoAlg}(\mathcal{C})$, the functor $M \otimes_{\mathcal{C}} \bullet: \mathcal{C} \rightarrow \mathcal{C}$ is endowed with a canonical (lax) ${ }^{11}$ tensor structure

$$
J_{V W}: M \otimes_{\mathcal{C}}\left(V \otimes_{\mathcal{C}} W\right) \rightarrow\left(M \otimes_{\mathcal{C}} V\right) \otimes_{\mathcal{C}}\left(M \otimes_{\mathcal{C}} W\right), \quad J_{V W}=A_{\mathcal{C}} \circ \text { id } \otimes \Delta,
$$

where $A_{\mathcal{C}}$ is the canonical isomorphism in $\mathcal{C}$ between $\left(M \otimes_{\mathcal{C}} M\right) \otimes_{\mathcal{C}}\left(V \otimes_{\mathcal{C}} W\right)$ and $\left(M \otimes_{\mathcal{C}} V\right) \otimes_{\mathcal{C}}\left(M \otimes_{\mathcal{C}} W\right)$.

8.1.2. Let $\left(\mathcal{D}, \otimes_{\mathcal{D}}, \mathbf{1}_{\mathcal{D}}, \boldsymbol{\Phi}_{\mathcal{D}}, \beta_{\mathcal{D}}\right)$ be a braided monoidal category and $G: \mathcal{C} \rightarrow \mathcal{D}$ a braided monoidal functor. Then it is possible to describe necessary and sufficient conditions for the composition functor $G_{M}=G \circ M \otimes_{\mathcal{C}} \bullet: \mathcal{C} \rightarrow \mathcal{D}$ to be a tensor functor. These conditions motivate the notion of $M$-adapted functor [27, Definition 1]. Specifically, $G$ is $M$-adapted if

(i) the composition $u_{M}$

$$
G(M) \stackrel{G\left(\epsilon_{M}\right)}{\longrightarrow} G\left(\mathbf{1}_{\mathcal{C}}\right) \simeq \mathbf{1}_{\mathcal{D}}
$$

is an isomorphism;

\footnotetext{
${ }^{11}$ i.e., $J_{V W}$ is not necessarily invertible.
} 
(ii) for every $V, W \in \mathcal{C}$, the composition $\tau_{V W}$

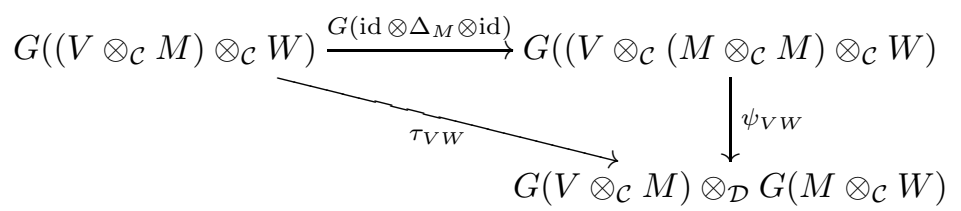

where $\psi_{V W}$ is the composition of the tensor structure on $G$ with the canonical isomorphism in $\mathcal{C}$ between the bracketing $\bullet(\bullet \bullet) \bullet$ and $(\bullet \bullet)(\bullet \bullet)$, is an isomorphism.

It follows that $G$ is $M$-adapted if and only if $G_{M}$ is a tensor functor.

8.1.3. For any $M$-adapted functor $G: \mathcal{C} \rightarrow \mathcal{D}, G_{M}(M)$ has a natural structure of Hopf algebra in $\mathcal{D}$. Specifically,

(i) the coalgebra structure is induced by that of $M$, with coproduct $G_{M}(M) \rightarrow$ $G_{M}(M) \otimes G_{M}(M)$

$$
J_{G} \circ G\left(A_{\mathcal{C}} \circ \Delta_{M} \otimes \Delta_{M}\right)
$$

and counit $G\left(\epsilon_{M} \otimes \epsilon_{M}\right)$;

(ii) the algebra structure is defined by the product $G_{M}(M) \otimes G_{M}(M) \rightarrow$ $G_{M}(M)$

and unit $G\left(\Delta_{M}\right) \circ u_{M}^{-1}$;

$$
G\left(\mathrm{id} \otimes \epsilon_{M} \otimes \mathrm{id}\right) \circ \tau_{M M}^{-1}
$$

(iii) finally, the antipode is given by $G\left(\left(\beta_{\mathcal{C}}\right)_{M M}^{-1}\right)$.

Moreover $G_{M}(M)$ acts and coacts on any $G_{M}(V), V \in \mathcal{C}$, with action

$$
G\left(\mathrm{id} \otimes \epsilon_{M} \otimes \mathrm{id}\right) \circ \tau_{M V}^{-1}
$$

and coaction

$$
R_{M V} \circ 1_{G_{M}(M)} \otimes \mathrm{id}
$$

where $R_{M V}$ is defined by

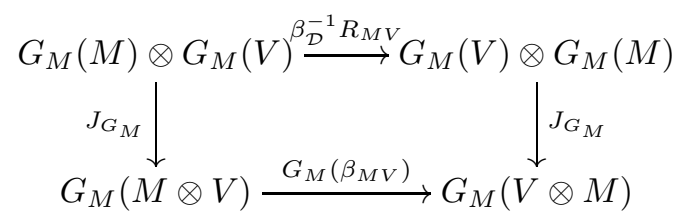

One verifies that (8.5) and (8.6) are compatible and therefore $G_{M}$ factors through the category of Drinfeld-Yetter $G_{M}(M)$-modules.

8.1.4. Finally, this construction applies to the case of Lie bialgebras. Set $\mathcal{D}=$ Vect $_{\mathfrak{k}}, \mathcal{C}=\mathrm{DY}_{\mathfrak{b}}^{\Phi}$, and consider the cocommutative coalgebra object in $D Y_{\mathfrak{b}}^{\Phi}$ given by the Verma module $M=M_{-}$. One can easily check that the functor of coinvariants $G: \mathrm{DY}_{\mathfrak{b}}^{\Phi} \rightarrow$ Vect $_{\mathfrak{k}}, G(V)=V_{\mathfrak{b}}$ is a $M$-adapted lax braided tensor functor. Moreover, there is a natural identification

$$
\phi_{V}: G_{M}(V)=(M \otimes V)_{\mathfrak{b}} \rightarrow V,
$$

where $\phi_{V}([x \otimes v])=S_{0}(x) v$ and $\phi_{V}^{-1}(v)=[1 \otimes v]$. It is easy to check that the Hopf algebra $G_{M}(M)$ is a quantisation of the Lie bialgebra $\mathfrak{b}$. 
This is easily reproduced in the PROP LBA $\llbracket \hbar \rrbracket$, giving rise to another universal quantisation functor. The advantage with respect to the Etingof-Kazhdan quantisation is that the construction of $G_{M}(M)$ does not require to consider topological Drinfeld-Yetter modules. On the other hand, one can easily see that this is equivalent to the Etingof-Kazhdan functor on the category of discrete Drinfeld-Yetter $\mathfrak{b}$-modules, as for any discrete $V \in \mathrm{DY}_{\mathfrak{b}}^{\Phi}$, there is a canonical isomorphism of tensor functors

given by the identifications

$$
G_{M}(V) \simeq F_{\mathfrak{b}}\left(V^{*}\right)^{*}
$$

$$
(M \otimes V)_{\mathfrak{b}} \simeq \operatorname{Hom}_{\mathfrak{b}}^{\mathfrak{b}}\left(M_{-} \otimes V, M_{+}^{\vee}\right)^{*} \simeq \operatorname{Hom}_{\mathfrak{b}}^{\mathfrak{b}}\left(M_{-}, M_{+}^{\vee} \otimes V^{*}\right)^{*} .
$$

8.2. Tensor structure on the restriction functor. The construction of the fiber functor $G_{M}: \mathrm{DY}_{\mathfrak{b}}^{\Phi} \rightarrow$ Vect $_{\mathrm{k}}$ generalises to the relative case obtaining analogous results to Theorems 3.1 and 5.1 .

8.2.1. For any split pair $\mathfrak{a} \rightarrow \mathfrak{b} \rightarrow \mathfrak{a}$, one can consider the Drinfeld-Yetter $\mathfrak{b}-$ module $L=S \mathrm{~m}$. This is endowed with a canonical structure of cocommutative coalgebra in $\mathrm{DY}_{\mathfrak{b}}^{\Phi}$, so that the functor $L \otimes \bullet: \mathrm{DY}_{\mathfrak{b}}^{\Phi} \rightarrow \mathrm{DY}_{\mathfrak{b}}^{\Phi}$ is naturally endowed with a lax tensor structure. Then one replaces the functor $G_{\mathfrak{b}}$ of $\mathfrak{b}$-coinvariants with

$$
G_{\mathfrak{m}}: \mathrm{DY}_{\mathfrak{b}}^{\Phi} \rightarrow \mathrm{DY}_{\mathfrak{a}}^{\Phi}, \quad G_{\mathfrak{m}}(V)=V_{\mathfrak{m}}
$$

One sees immediately that $G_{\mathfrak{m}}$ is a lax braided tensor functor, which is adapted to $L$. Moreover, there is a canonical isomorphism

$$
\phi_{V}: G_{\mathfrak{m}}(L \otimes V) \rightarrow V
$$

where $\phi_{V}([x \otimes v])=S_{0}(x) v$ and $\phi_{V}^{-1}(v)=[1 \otimes v]$. It follows that

(i) the functor $G_{L}(V)=(L \otimes V)_{\mathfrak{m}}$ from $\mathrm{DY}_{\mathfrak{b}}^{\Phi}$ to $\mathrm{DY}_{\mathfrak{a}}^{\Phi}$ is isomorphic to $\operatorname{Res}_{\mathfrak{a}, \mathfrak{b}}$ and is naturally endowed with a tensor structure;

(ii) $G_{L}(L)$ has a Hopf algebra structure defined by the formulae (8.4), (8.3), corresponding to $L$ and $G_{\mathfrak{m}}$;

(iii) $G_{L}(L)$ acts and coacts by (8.5), (8.6), on any $G_{L}(V), V \in \mathrm{DY}_{\mathfrak{b}}^{\Phi}$ and the functor $G_{L}$ factors through the category of Drinfeld-Yetter $G_{L}(L)$ modules in $\mathrm{DY} \mathbf{a}_{\mathfrak{a}}^{\Phi}$.

As in the case $\mathfrak{a}=0$, restricted to the category of discrete Drinfeld-Yetter $\mathfrak{b}$ modules, there is an isomorphism of tensor functors

$$
G_{L}(V) \simeq F_{\mathfrak{a}, \mathfrak{b}}\left(V^{*}\right)^{*},
$$

given by the identifications

$$
(L \otimes V)_{\mathfrak{m}} \simeq \operatorname{Hom}_{\mathfrak{b}}^{\mathfrak{b}}\left(L_{-} \otimes V, N_{+}^{\vee}\right)^{*} \simeq \operatorname{Hom}_{\mathfrak{b}}^{\mathfrak{b}}\left(L_{-}, N_{+}^{\vee} \otimes V^{*}\right)^{*} .
$$

8.2.2. The tensor functor $G_{L}$ has an obvious propic realisation in PLBA $\hbar \rrbracket$, which relies on the identification (8.8) and leads to an analogue of Theorem 7.11 (i)-(iii). Namely, for every $V, W \in \mathrm{DY}_{[\mathfrak{[ b}]}^{\Phi}$, one defines the twist $J_{G_{L}}$ as the composition

$$
V \otimes W \stackrel{\phi_{V \otimes W}^{-1}}{\longrightarrow} L \otimes V \otimes W \stackrel{A_{\Phi} \circ \Delta \otimes \mathrm{id}^{\otimes 2}}{\longrightarrow} L \otimes V \otimes L \otimes W \stackrel{\phi_{V} \otimes \phi_{W}}{\longrightarrow} V \otimes W,
$$

where $A_{\Phi}$ denotes the usual isomorphism from $(L \otimes L) \otimes(V \otimes W)$ to $(L \otimes V) \otimes(L \otimes W)$. The Hopf algebra structure on $G_{L}(L)$, its action and coaction on any DrinfeldYetter $[\mathfrak{b}]$-module have their analogues in PLBA $\llbracket \hbar \rrbracket$. 
In particular, from (8.9) we get the following

Proposition. The tensor structure $J_{\mathfrak{a}, \mathfrak{b}}$, constructed in Sections $\S 3$ and $\S 7$, is gauge equivalent to a universal relative tensor structure from PLBA $₫ \rrbracket$.

Finally, we point out that the proof of Theorems 1.6 and 1.7 does not get any simpler in this context. Namely, in order to obtain an alternative proof, it would be necessary to construct a natural transformation of tensor functors

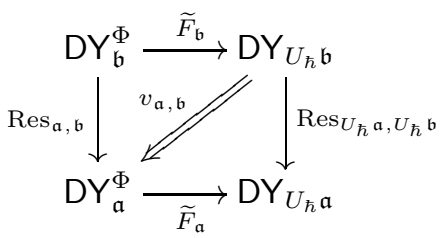

where the tensor structure on the restriction functors $\operatorname{Res}_{\mathfrak{a}, \mathfrak{b}}$ and $\operatorname{Res}_{U_{\hbar} \mathfrak{a}, U_{\hbar} \mathfrak{b}}$ are now induced by the identifications

$$
\operatorname{Res}_{\mathfrak{a}, \mathfrak{b}}(V) \simeq(L \otimes V)_{\mathfrak{m}} \quad \text { and } \quad \operatorname{Res}_{U_{\hbar} \mathfrak{a}, U_{\hbar} \mathfrak{b}}(\mathcal{V}) \simeq\left(L^{\hbar} \otimes \mathcal{V}\right)_{L^{\hbar}}
$$

Adapting the proof of Theorem 4.14, it would be necessary to show that the spaces of coinvariants are preserved by the quantisation as Drinfeld-Yetter modules. The most obvious strategy to approach the problem relies on the equivalences $\widetilde{F}_{\mathfrak{b}}$ and $\widetilde{F}_{\mathfrak{a}}$ and therefore on the identifications

$$
\begin{aligned}
(L \otimes V)_{\mathfrak{m}} & \simeq \operatorname{Hom}_{\mathfrak{b}}^{\mathfrak{b}}\left(L_{-}, N_{+}^{\vee} \otimes V^{*}\right)^{*} \\
\left(L^{\hbar} \otimes \mathcal{V}\right)_{L^{\hbar}} & \simeq \operatorname{Hom}_{U_{\hbar} \mathfrak{b}}^{U_{\hbar} \mathfrak{b}}\left(L_{-}^{\hbar},\left(N_{+}^{\vee}\right)^{\hbar} \otimes \mathcal{V}^{*}\right)^{*} .
\end{aligned}
$$

Ultimately, it becomes necessary, exactly as in $\S 4.14$, to identify the quantisation of the relative Verma modules with their quantum analogues, producing the same proof we followed in Section $\S 4$.

\section{Appendix A. Quantum double and Drinfeld-Yetter modules}

Let $B$ be a finite-dimensional Hopf algebra or a quantised enveloping algebra. We review in this section the construction of the quantum double of $B$ as a double cross product of Hopf algebras, following [25, §7.2]. We also describe the equivalence of braided tensor categories between (admissible) Drinfeld-Yetter modules over $B$ and modules over its quantum double.

A.1. Double cross product Hopf algebras [25]. Two Hopf algebras ( $B, m_{B}$, $\left.\iota_{B}, \Delta_{B}, \varepsilon_{B}, S_{B}\right)$ and $\left(C, m_{C}, \iota_{C}, \Delta_{C}, \varepsilon_{C}, S_{C}\right)$ form a matched pair if they are endowed with maps

$$
\triangleright: B \otimes C \rightarrow C \quad \text { and } \quad \triangleleft: B \otimes C \rightarrow B
$$

such that

(i) $(C, \triangleright)$ is a left $B$-module coalgebra, i.e.,

$$
\Delta_{C} \circ \triangleright=\triangleright \otimes \triangleright \circ(23) \circ \Delta_{B} \otimes \Delta_{C} ;
$$

(ii) $(B, \triangleleft)$ is a right $C$-module coalgebra, i.e.,

$$
\Delta_{B} \circ \triangleleft=\triangleleft \otimes \triangleleft \circ(23) \circ \Delta_{B} \otimes \Delta_{C} ;
$$


(iii) $\triangleleft$ and $\triangleright$ are compatible, respectively, with $m_{B}$ and $m_{C}$, i.e.,

$$
\begin{aligned}
& \triangleleft \circ m_{B} \otimes \mathrm{id}=m_{B} \circ \triangleleft \otimes \mathrm{id} \circ \mathrm{id} \otimes \triangleright \otimes \triangleleft \circ(34) \circ \mathrm{id} \otimes \Delta_{B} \otimes \Delta_{C}, \\
& \triangleright \circ \mathrm{id} \otimes m_{C}=m_{C} \circ \mathrm{id} \otimes \triangleright \circ \triangleleft \otimes \triangleright \otimes \mathrm{id} \circ(23) \circ \Delta_{B} \otimes \Delta_{C} \otimes \mathrm{id} ;
\end{aligned}
$$

(iv) the units $\iota_{B}$ and $\iota_{C}$ are module maps, i.e.,

$$
\begin{aligned}
& \triangleleft \circ \iota_{B} \otimes \mathrm{id}=\iota_{B} \otimes \varepsilon_{C}, \\
& \triangleright \circ \mathrm{id} \otimes \iota_{C}=\varepsilon_{B} \otimes \iota_{C} ;
\end{aligned}
$$

(v) finally, $\triangleleft$ and $\triangleright$ satisfy the following compatibility condition:

$$
\triangleleft \otimes \triangleright \circ(23) \circ \Delta_{B} \otimes \Delta_{C}=\triangleleft \otimes \triangleright \circ(23) \circ \Delta_{B}^{21} \otimes \Delta_{C}^{21}
$$

Any matched pair $(B, C, \triangleright, \triangleleft)$ gives rise to a Hopf algebra structure on $B \otimes C$, called the double cross product of $B$ and $C$, and denoted $B \bowtie C$. The product is defined by requiring that $B \bowtie C$ contains $B, C$ as subalgebras under the natural inclusions $i_{B}: B \rightarrow B \otimes C$ and $i_{C}: C \rightarrow B \otimes C$, and

$$
\begin{aligned}
& m_{B, C} \circ i_{B} \otimes i_{C}=i d_{B \otimes C}, \\
& m_{B, C} \circ i_{C} \otimes i_{B}=\triangleleft \otimes \triangleright \circ(23) \circ \Delta_{B} \otimes \Delta_{C} \circ(12) .
\end{aligned}
$$

The remaining structure is defined as follows:

$$
\begin{aligned}
\iota_{B, C} & =\iota_{B} \otimes \iota_{C}, \\
\Delta_{B, C} & =(23) \circ \Delta_{B} \otimes \Delta_{C}, \\
\varepsilon_{B, C} & =\varepsilon_{B} \otimes \varepsilon_{C}, \\
S_{B, C} & =m_{B, C} \circ i_{C} \otimes i_{B} \circ S_{C} \otimes S_{B} \circ(12) .
\end{aligned}
$$

In particular, $B \bowtie C$ contains $B$ and $C$ as Hopf subalgebras.

A.2. Quantum double. Let $B$ be a finite-dimensional Hopf algebra. The quantum double $D B$ (cf. [7] and $\S 2.18$ ) has a natural description as a double cross product Hopf algebra. Namely, let $\triangleright_{B}: B \otimes B^{*} \rightarrow B^{*}$ be the left coadjoint action of $B$ on $B^{*}$, i.e.,

$$
\triangleright_{B}=\langle,\rangle \otimes \mathrm{id} \circ \mathrm{id} \otimes m_{B^{*}} \otimes \mathrm{id} \circ(234) \circ \mathrm{id} \otimes S_{B^{*}}^{-1} \otimes \mathrm{id}^{\otimes 2} \circ \mathrm{id} \otimes \Delta_{B^{*}}^{(3)}
$$

and $\triangleleft_{B}: B \otimes B^{*} \rightarrow B$ the right coadjoint action of $B^{*}$ on $B$, i.e.,

$$
\triangleleft_{B}=\mathrm{id} \otimes\langle,\rangle \circ \mathrm{id} \otimes m_{B} \otimes \mathrm{id} \circ(321) \circ \mathrm{id}^{\otimes 2} \otimes S_{B}^{-1} \otimes \mathrm{id} \circ \Delta_{B}^{(3)} \otimes \mathrm{id} .
$$

Then, the product on $D B$ given by (2.8) reads

$$
m_{D B} \circ i_{B^{*}} \otimes i_{B}=\triangleleft_{B} \otimes \triangleright_{B} \circ(23) \circ \Delta_{B} \otimes \Delta_{B^{*}}^{21} \circ(12) .
$$

In particular, it follows that $\left(B, B^{\circ}, \triangleright_{B}, \triangleleft_{B}\right)$, where $B^{\circ}=B^{* \text { cop }}$, is a matched pair of Hopf algebras and $D B=B \bowtie B^{\circ}$ is the associated double cross product Hopf algebra. 
A.3. Drinfeld-Yetter modules revisited. Let $\mathbf{\nabla}_{B}: B \otimes B \rightarrow B$ and $\boldsymbol{\Delta}_{B}: B \rightarrow$ $B \otimes B$ be, respectively, the adjoint action and the adjoint coaction of $B$ on itself (cf. (4.3), (4.1)), i.e.,

$$
\begin{aligned}
& \mathbf{\Delta}_{B}=m_{B} \otimes \mathrm{id} \circ(123) \circ S_{B}^{-1} \otimes \mathrm{id}^{\otimes 2} \circ \Delta_{B}^{(3)}, \\
& \mathbf{v}_{B}=m_{B}^{(3)} \circ(321) \circ S_{B}^{-1} \otimes \mathrm{id}^{\otimes 2} \circ \Delta_{B} \otimes \mathrm{id} .
\end{aligned}
$$

Then, for every Drinfeld-Yetter $B$-module $\left(V, \pi_{V}, \pi_{V}^{*}\right)$, the compatibility condition (2.7) between the action and the coaction reads

$$
\pi_{V}^{*} \circ \pi_{V}=m_{B} \otimes \pi_{V} \circ(321) \circ \mathbf{\Lambda}_{B} \otimes \mathbf{\nabla}_{B} \otimes \mathrm{id} \circ \Delta_{B} \otimes \pi_{V}^{*} .
$$

A.4. Drinfeld-Yetter modules and quantum double. We mentioned in $\S 2.18$ that the category of $D B$-modules is equivalent to the category of Drinfeld-Yetter $B$-modules. This follows, in particular, from the duality between the maps $\triangleleft_{B}$ (resp. $\triangleright_{B}$ ) and $\boldsymbol{\Delta}_{B}$ (resp. $\boldsymbol{\nabla}_{B}$ ), appearing in the formula (A.1) for the product of $D B$ and in the compatibility condition (A.2) for Drinfeld-Yetter $B$-modules. Indeed, the coadjoint action of $B^{*}$ on $B$ corresponds to the adjoint coaction of $B$ on $B$, i.e.,

$$
\begin{aligned}
& \triangleleft_{B}=\operatorname{id}_{B} \otimes\langle,\rangle \circ(23) \circ \mathbf{\Delta}_{B} \otimes \operatorname{id}_{B^{*}}, \\
& \mathbf{\Delta}_{B}=(12) \circ \triangleleft_{B} \otimes \operatorname{id}_{B} \circ \operatorname{id}_{B} \otimes R_{B}^{21},
\end{aligned}
$$

where $\langle$,$\rangle denotes the pairing between B$ and $B^{*}$ and $R_{B} \in B \otimes B^{*}$ is the $R$-matrix. Similarly, the coadjoint action of $B$ on $B^{*}$ corresponds to the adjoint action of $B$ on $B$, i.e.,

$$
\begin{aligned}
\langle,\rangle^{21} \circ \operatorname{id}_{B^{*}} \otimes \mathbf{\nabla}_{B} & =\langle,\rangle^{21} \circ \triangleright_{B} \otimes \operatorname{id}_{B} \circ(12), \\
\nabla_{B} \otimes \operatorname{id}_{B^{*}} \circ \operatorname{id}_{B} \otimes R_{B} & =\operatorname{id}_{B} \otimes \triangleright_{B} \circ(23) \circ R_{B} \otimes \operatorname{id}_{B} .
\end{aligned}
$$

Theorem. There is a canonical equivalence of braided tensor categories

$$
\mathrm{DY}_{B} \stackrel{\Xi}{\rightleftarrows} \operatorname{Rep} D B
$$

where

(i) for any Drinfeld-Yetter $B$-module $\left(V, \pi_{V}, \pi_{V}^{*}\right), \Xi\left(V, \pi_{V}, \pi_{V}^{*}\right)=\left(V, \xi_{V}\right)$ with

$$
\xi_{V}=\pi_{V} \circ \mathrm{id}_{B} \otimes\langle,\rangle^{21} \otimes \operatorname{id}_{V} \circ \mathrm{id}_{B} \otimes \mathrm{id}_{B^{*}} \otimes \pi_{V}^{*} ;
$$

(ii) for any $D B$-module $\left(V, \xi_{V}\right), \Theta\left(V, \xi_{V}\right)=\left(V, \pi_{V}, \pi_{V}^{*}\right)$ with

$$
\begin{aligned}
& \pi_{V}=\xi_{V} \circ i_{B} \otimes \operatorname{id}_{V}, \\
& \pi_{V}^{*}=\operatorname{id}_{B} \otimes \xi_{V} \circ \operatorname{id}_{B} \otimes i_{B^{*}} \otimes \operatorname{id}_{V} \circ R_{B} \otimes \operatorname{id}_{V} .
\end{aligned}
$$

Proof. The only non trivial part consists in showing that the functors $\Theta$ and $\Xi$ are well-defined. It is then clear that they are inverse of each other and preserve the braided tensor structures on $\mathrm{DY}_{B}$ and $\operatorname{Rep} D B$.

(i) We have to show that $\xi_{V}$ defines an action of $D B$ on $V$. Namely, we need to check that,

$$
\xi_{V} \circ \mathrm{id}_{D B} \otimes \xi_{V} \circ i_{B^{*}} \otimes i_{B} \otimes \mathrm{id}_{V}=\xi_{V} \circ m_{D B} \otimes \operatorname{id}_{V} \circ i_{B^{*}} \otimes i_{B} \otimes \mathrm{id}_{V} .
$$


By definition of $\xi_{V}$, the LHS reads

$$
\begin{aligned}
\xi_{V} \circ \mathrm{id}_{D B} \otimes \xi_{V} \circ i_{B^{*}} \otimes i_{B} \otimes \mathrm{id}_{V} \\
\quad=\langle,\rangle^{21} \otimes \mathrm{id}_{V} \circ \pi_{V}^{*} \circ \mathrm{id}_{B^{*}} \otimes \pi_{V} \\
\quad=\langle,\rangle^{21} \otimes \mathrm{id}_{V} \circ \mathrm{id}_{B^{*}} \otimes\left(\pi_{V}^{*} \circ \pi_{V}\right) \\
\quad=\langle,\rangle^{21} \otimes \mathrm{id}_{V} \circ \mathrm{id}_{B^{*}} \otimes\left(m_{B} \otimes \pi_{V} \circ(321) \circ \mathbf{\Delta}_{B} \otimes \mathbf{\nabla}_{B} \otimes \mathrm{id} \circ \Delta_{B} \otimes \pi_{V}^{*}\right) \\
\quad=\left(\langle,\rangle^{21} \circ \operatorname{id}_{B^{*}} \otimes\langle,\rangle^{21} \otimes \mathrm{id}_{B}\right) \otimes \pi_{V} \circ(45) \circ \mathrm{id}_{B^{*}}^{\otimes 2} \otimes \mathbf{\Delta}_{B} \otimes \mathbf{\nabla}_{B} \circ \Delta_{B^{*}} \otimes \Delta_{B} \otimes \pi_{V}^{*} .
\end{aligned}
$$

Applying (A.3) and (A.5), we get

$$
\begin{aligned}
& \xi_{V} \circ \operatorname{id}_{D B} \otimes \xi_{V} \circ i_{B^{*}} \otimes i_{B} \otimes \operatorname{id}_{V} \\
& =\pi_{V} \circ \mathrm{id}_{B} \otimes\langle,\rangle^{21} \otimes \mathrm{id}_{V} \circ \triangleleft_{B} \otimes \triangleright_{B} \otimes \mathrm{id}_{B} \otimes \mathrm{id}_{V} \circ(431) \circ \Delta_{B^{*}} \otimes \Delta_{B} \otimes \pi_{V}^{*} \\
& =\pi_{V} \circ \mathrm{id}_{B} \otimes\langle,\rangle^{21} \otimes \mathrm{id}_{V} \circ \mathrm{id}_{B} \otimes \operatorname{id}_{B^{*}} \otimes \pi_{V}^{*} \circ \triangleleft_{B} \otimes \triangleright_{B} \otimes \mathrm{id}_{V} \circ(23) \circ \\
& \circ \Delta_{B} \otimes \Delta_{B^{*}}^{21} \otimes \operatorname{id}_{V} \circ(12) \\
& =\xi_{V} \circ m_{D B} \otimes \operatorname{id}_{V} \circ i_{B^{*}} \otimes i_{B} \otimes \operatorname{id}_{V}
\end{aligned}
$$

as required.

(ii) It is clear that $\pi_{V}$ and $\pi_{V}^{*}$ define an action and a coaction of $B$ on $V$. We have to show that they satisfy the compatibility condition (A.2). One has

$$
\begin{aligned}
& \pi_{V}^{*} \circ \pi_{V}=\mathrm{id}_{B} \otimes \xi_{V} \circ \mathrm{id}_{B} \otimes \mathrm{id}_{B^{*}} \otimes \xi_{V} \circ R_{B} \otimes \mathrm{id}_{B} \otimes \mathrm{id}_{V} \\
& =\mathrm{id}_{B} \otimes \xi_{V} \circ \mathrm{id}_{B} \otimes m_{D B} \otimes \mathrm{id}_{V} \circ R_{B} \otimes \mathrm{id}_{B} \otimes \mathrm{id}_{V} \\
& =m_{B} \otimes \triangleleft_{B} \otimes \triangleright_{B} \otimes \mathrm{id}_{V} \circ(6534) \circ \mathrm{id}^{\otimes 4} \otimes \Delta_{B} \otimes \mathrm{id}_{V} \circ \\
& \mathrm{id}_{B} \otimes R_{B} \otimes \operatorname{id}_{B^{*}} \otimes \operatorname{id}_{B} \otimes \operatorname{id}_{V} \circ R_{B} \otimes \operatorname{id}_{B} \otimes \operatorname{id}_{V} .
\end{aligned}
$$

Applying (A.4) and (A.6), we get

$$
\pi_{V}^{*} \circ \pi_{V}=m_{B} \otimes \pi_{V} \circ(321) \circ \boldsymbol{\Delta}_{B} \otimes \mathbf{v}_{B} \otimes \mathrm{id} \circ \Delta_{B} \otimes \pi_{V}^{*}
$$

as required.

A.5. QUE algebras. Let now $B$ be a QUE algebra, and $B^{\prime} \subset B$ the corresponding QFSH algebra (see $\S 2.19$ ). In $\S 4.3$ and $\S 4.4$, we proved the following

\section{Proposition.}

(i) The adjoint action of $B$ on itself preserves $B^{\prime}$. In particular, $\left(B^{\prime}, \mathbf{v}_{B}\right)$ is a left B-module.

(ii) The adjoint coaction of $B$ on itself factors through $B^{\prime}$. In particular, $\left(B, \mathbf{\Lambda}_{B}\right)$ is an admissible right $B$-comodule.

Set $B^{\vee}=\left(B^{\prime}\right)^{* \text { cop }}$. The adjoint action of $B$ on $B^{\prime}$ induces a coadjoint action $\triangleright_{B}: B \otimes B^{\vee} \rightarrow B^{\vee}$ of $B$ on $B^{\vee}$. Similarly, the adjoint coaction of $B^{\prime}$ on $B$ induces a coadjoint action $\triangleleft_{B}: B \otimes B^{\vee} \rightarrow B$ of $B^{\vee}$ on $B$. One checks easitly that the tuple $\left(B, B^{\vee}, \triangleright_{B}, \triangleleft_{B}\right)$ is a matched pair of Hopf algebras and the QUE quantum double of $B$ is precisely the double cross product $D B=B \bowtie B^{\vee}$.

The computations carried out in $\S$ A.2- $\$$ A.4 apply verbatim to this case and yield the following.

Theorem. The formulae from Theorem A.4 give an equivalence of braided tensor categories between the category DY ${ }_{B}^{\mathrm{adm}}$ of admissible Drinfeld-Yetter B-modules and the category Rep DB of modules over the quantum double. 


\section{REFERENCES}

[1] A. Appel, V. Toledano Laredo, Quasi-Coxeter categories and a relative Etingof-Kazhdan quantisation functor, arXiv:1212.6720, 63 pp.

[2] A. Appel, V. Toledano Laredo, Quasi-Coxeter categories and quantum groups, arXiv:1610.09741, 48 pp.

[3] A. Appel, V. Toledano Laredo, Uniqueness of quasi-Coxeter structures on Kac-Moody algebras, arXiv:1508.01945, $61 \mathrm{pp}$.

[4] A. Appel, V. Toledano Laredo, Monodromy of the Casimir connection of a symmetrisable Kac-Moody algebra, arXiv:1512.03041, 48 pp.

[5] A. Brochier, A Duflo star-product for Poisson groups, SIGMA Symmetry Integrability Geom. Methods Appl. 12 (2016), Paper No. 088, 12 pp.

[6] G. Cortiñas, An explicit formula for PBW quantization, Comm. Algebra 30 (2002), $1705-1713$.

[7] V. G. Drinfeld, Quantum groups, Proceedings of the International Congress of Mathematicians, (Berkeley, Calif., 1986), 798-820, Amer. Math. Soc., 1987.

[8] V. G. Drinfeld, Quasi-Hopf algebras and the KZ equations, Problems of modern quantum field theory (Alushta, 1989), 1-13, Res. Rep. Phys., Springer, 1989.

[9] V. G. Drinfeld, Quasi-Hopf Algebras, Leningrad Math. J. 1 (1990), 1419-1457.

[10] B. Enriquez, A cohomological construction of quantization functors of Lie bialgebras, Adv. Math. 197 (2005), no. 2, 430-479.

[11] B. Enriquez, P. Etingof, On the invertibility of quantization functors, J. Algebra 289 (2005), 321-345.

[12] B. Enriquez, N. Geer, Compatibility of quantization functors of Lie bialgebras with duality and doubling operations, Selecta Math. (N.S.) 15 (2009), 1-59.

[13] P. Etingof, personal communication, 2017.

[14] P. Etingof, D. Kazhdan, Quantization of Lie bialgebras, I, Selecta Math. (N.S.) 2 (1996), $1-41$.

[15] P. Etingof, D. Kazhdan, Quantization of Lie bialgebras, II, Selecta Math. (N.S.) 4 (1998), 213-231.

[16] P. Etingof, D. Kazhdan, Quantization of Lie bialgebras. VI. Quantization of generalized Kac-Moody algebras, Transform. Groups 13 (2008), 527-539.

[17] P. Etingof, O. Schiffman, Lectures on quantum groups. Second edition. Lectures in Mathematical Physics. International Press, Somerville, MA, 2002.

[18] F. Gavarini, The quantum duality principle, Ann. Inst. Fourier (Grenoble) 52 (2002), 809-834.

[19] C. Kassel, Quantum groups. Graduate Texts in Mathematics, 155. Springer-Verlag, New York, 1995.

[20] S. Lack, A 2-categories companion, Towards higher categories, 105-191, IMA Vol. Math. Appl., 152, Springer, 2010.

[21] F. W. Lawvere, Functorial semantics of algebraic theories, Proc. Nat. Acad. Sci. U.S.A. 50 (1963), 869-872.

[22] G. Lusztig, Introduction to quantum groups. Birkhäuser Boston, 1993.

[23] S. MacLane, Categorical algebra, Bull. Amer. Math. Soc. 71 (1965), 40-106.

[24] S. Majid, Cross products by braided groups and bosonization, J. Algebra 163 (1994),165190.

[25] S. Majid, Foundations of Quantum Group Theory, Cambridge University Press, 1995.

[26] D. Radford, The structure of Hopf algebras with a projection, J. Algebra 92 (1985), 322-347.

[27] P. Ševera, Quantization of Lie bialgebras revisited, Selecta Math. (N.S.) 22 (2016), 15631581.

[28] Y. Sommerhäuser, Yetter-Drinfel'd Hopf algebras over groups of prime order. Lecture Notes in Mathematics, 1789. Springer-Verlag, Berlin, 2002.

[29] V. Toledano Laredo, Quasi-Coxeter algebras, Dynkin diagram cohomology and quantum Weyl groups, Int. Math. Res. Pap. IMRP 2008, art. ID rpn009, 167 pp.

[30] V. Toledano Laredo, Quasi-Coxeter quasitriangular quasibialgebras and the Casimir connection, arXiv:1601.04076, 55 pp. 
[31] D. N. Yetter, Quantum groups and representations of monoidal categories, Math. Proc. Cambridge Philos. Soc. 108 (1990), 261-290.

School of Mathematics, University of Edinburgh, James Clark Maxwell Building, Peter Guthrie Tait Road, Edinburgh, EH9 3FD, UK

E-mail address: andrea.appel@ed.ac.uk

Department of Mathematics, Northeastern University, 360 Huntington Avenue, Boston MA 02115

E-mail address: V.ToledanoLaredo@neu.edu 